\title{
Comparative analysis of organ size, shape, and patterning in diverse species
}

\author{
Dissertation \\ for the award of the degree \\ "Doctor of Philosophy" \\ Division of Mathematics and Natural Sciences \\ of the Georg-August-Universität Göttingen
}

within the doctoral program Genes and Development of the Göttingen Graduate School for Neurosciences, Biophysics, and Molecular Biosciences of the Georg-August University School of Science (GAUSS)

submitted by

\section{Natalia Siomava}

from Kurganinsk, Russian Federation

Göttingen 2016 


\title{
Thesis Committee
}

Prof. Dr Ernst A. Wimmer Department of Developmental Biology,

Johann-Friedrich-Blumenbach-Institute of Zoology and Anthropology,

Georg-August-University Göttingen

PD Dr. Ronald P. Kühnlein Department of Molecular Developmental Biology,

Max Planck Institute for Biophysical Chemistry

Prof. Dr. Andreas Wodarz Institute I of Anatomy,

University of Cologne

\section{Members of the Examination Board}

\author{
Referee: $\quad$ Prof. Dr Ernst A. Wimmer \\ Department of Developmental Biology, \\ Johann-Friedrich-Blumenbach-Institute of Zoology and Anthropology, \\ Georg-August-University Göttingen \\ $2^{\text {nd }}$ Referee: $\quad$ PD Dr. Ronald P. Kühnlein \\ Department of Molecular Developmental Biology, \\ Max Planck Institute for Biophysical Chemistry
}

\section{Further members of the Examination Board}

Prof. Dr. Andreas Wodarz, Institute I of Anatomy, University of Cologne

Dr. Nico Posnien, Department of Developmental Biology, Johann-Friedrich-BlumenbachInstitute of Zoology and Anthropology, Georg-August-University Göttingen

Prof. Dr. Gregor Bucher, Department of Developmental Biology, Johann-FriedrichBlumenbach-Institute of Zoology and Anthropology, Georg-August-University Göttingen

Prof. Dr. Reinhard Schuh, Department of Molecular Developmental Biolog, Max Planck Institute for Biophysical Chemistry

Date of oral examination: December 21 $1^{\text {st }}, 2016$ 


\section{Authorship Declaration}

I hereby declare that the dissertation "Comparative analysis of organ size, shape, and patterning in diverse species" was written by myself with no other sources and help than quoted. It is based on my experimental work in the department of Developmental Biology, Georg-August-University Göttingen. Apart from the publications listed below the thesis has not been published so far. It has not been submitted elsewhere for the award of any other doctoral degree.

Göttingen, October $15^{\text {th }}, 2016$

Natalia Siomava

Parts of this work that have already been published:

- Siomava N, Wimmer EA, Posnien N (2016) Size relationships of different body parts in the three dipteran species Drosophila melanogaster, Ceratitis capitata and Musca domestica. Dev Genes Evol 226: 245-56. Doi: 10.1007/s00427-016-0543-6

Manuscripts in preparation:

- Siomava N, Wimmer EA, Posnien N. Extensive sexual wing shape dimorphism in Drosophila melanogaster, Ceratitis capitata and Musca domestica. In preparation

- Siomava N, Wimmer EA. Conservation and divergence of gene expression patterns in wing imaginal discs of Ceratitis capitata and Musca domestica. In preparation

Gedruckt mit Unterstützung des Deutschen Akademischen Austauschdienstes 
Experientia est optima magistra

Опыт - лучший учитель

Experience is the best teacher

Erfahrung ist der beste Lehrmeister 
TABLE OF CONTENTS

1 SUMMARY.

2 GENERAL INTRODUCTION............................................................................................................. 3

2.1 Models to study tissue growth and patterning............................................................. 3

2.2 Dpp morphogen gradient........................................................................................ 4

2.3 Growth model for Drosophila wing imaginal discs................................................. 6

2.4 Generalization of the growth models....................................................................... 8

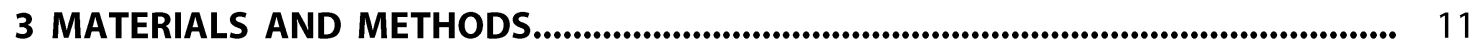

3.1 Fly stocks.................................................................................................................... 11

3.2 Generation of differently sized flies.............................................................................. 11

3.3 Estimation of size and shape parameters in Drosophila, Ceratitis, and Musca.

3.3.1 Photo imaging and landmark acquisition ......................................................................... 12

3.3.2 Estimation of size .............................................................................................................. 13

3.3.2.1 Measurements and computation of size estimators....................................... 13

3.3.2.2 Computation of allometric coefficients......................................................... 15

3.3.2.3 The relative wing size and wing loading.................................................... 15

3.3.2.4 Counting bristles on the wing membrane..................................................... 15

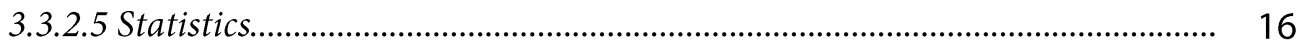

3.3.3. Wing morphometrics................................................................................................... 16

3.3.3.1 Procrustes superimposition....................................................................... 16

3.3.3.2 Estimation of growth trajectories.................................................................... 17

3.3.3.3 Interspecies comparison .............................................................................. 17

3.3.3.4 Intraspecies comparison: sexual dimorphism, temperature and density

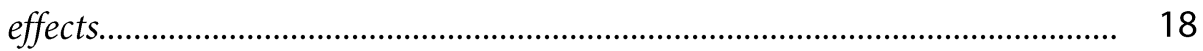

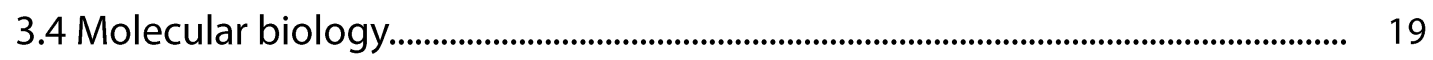

3.4.1 Oligonucleotides........................................................................................................ 19

3.4.2 Isolation of mRNA and synthesis of CDNA.................................................................... 19

3.4.3 Rapid amplification of CDNA ends................................................................................. 19

3.4.4 Cloning .................................................................................................................... 19

3.4.5 Transformation and plasmid DNA preparation .............................................................. 20 
3.5 Localization of gene expression in fly embryos and wing imaginal discs...... 20

3.5.1 Preparation and storage of embryos and larval tissue................................................... 20

3.5.2 Synthesis of digoxigenin-labeled RNA probes................................................................. 21

3.5.3 In situ hybridization ........................................................................................................ 21

3.5.4 Cryosectioning................................................................................................................ 22

3.6. Design of CRISPR/Cas9 system in Ceratitis and Musca.......................................... 22

3.6.1 Single guide RNA........................................................................................................ 22

3.6.2 Design of vectors for the homologous recombination.................................................. 23

4 BODY SIZE AS AN INTEGRAL FEATURE........................................................................... 25

4.1 Basis and importance of the size variation................................................................ 25

4.2 Variation of size parameters in different fly species................................................. 26

4.2.1 Correlation of wing parameters....................................................................................... 26

4.2.2 Sexual size dimorphism..................................................................................................... 28

4.2.3 Alterations of size in response to environmental cues................................................... 29

4.2.4 Response to changing environmental conditions is sex-dependent........................... $\quad 30$

4.2.5 Evolutionary and static allometries for thorax, tibia, and wing size........................... 32

4.2.6 Estimators of the absolute body size in Ceratitis capitata and Musca domestica......................................................................................................................... 33

4.2.7 Variation of the relative wing size .................................................................................... 34

4.2.8 Ceratitis wing are mainly bigger due to the cell size..................................................... 35

4.2.9 Wing loading...................................................................................................................... 36

4.3 Discussion and future work.................................................................................. 38

4.3.1 Estimation of the wing size.................................................................................................... 38

4.3.2 Sexual size dimorphism in Drosophila, Ceratitis, and Musca........................................ 38

4.3.3 Body and organ size: response to different rearing temperatures................................ 40

4.3.4 Body and organ size: response to changing rearing densities..................................... 41

4.3.5 Body size estimators and growth scaling....................................................................... 42

4.3.6 Variation of wing size and wing loading....................................................................... 43

5 VARIATION OF WING SHAPE IN DIFFERENT FLY SPECIES.......................................... 45

5.1 Geometric morphometrics of fly wings........................................................................ 45

5.2 Sexual shape dimorphism of Drosophila, Ceratitis, and Musca wings............. 46

5.2.1 Wing shape variation in dipteran species......................................................................... 47

5.2.2 Growth trajectories and static allometry..................................................................... 48

5.2.3 Sexual dimorphism in wing shape................................................................................ 53 
5.2.4 Sexual shape dimorphism in response to rearing temperatures................................. 54

5.2.5 Sexual shape dimorphism in response to different larval densities............................. 56

5.3 Discussion and future work......................................................................................... 58

5.3.1 Sexual shape dimorphism of Drosophila, Ceratitis, and Musca wings..................... 58

5.3.2 Sexual dimorphism in the size and shape relationships................................................. 59

5.3.3 Sexual shape dimorphism of wings in response to different environmental conditions s.................................................................................................................... 60

5.3.4 Potential functional implications of plasticity and sexual dimorphism in wing size and shape for mating behavior................................................................................ 61

6 GENE EXPRESSION IN FLY EMBRYOS AND WING IMAGINAL DISCS................... 65

6.1 Gene regulation in the developing fly wing............................................................. 65

6.1.1 Identification of homologous wing patterning genes in Drosophila, Ceratitis, and Musca........................................................................................................................... 68

6.1.2 Verification of predicted RNA isoforms in Ceratitis......................................................... 72

6.1.3 Verification of predicted RNA isoforms in Musca.............................................................. 73

6.2 Expression of genes involved in the wing development and patterning...... 75

6.2.1 Gene expression in wing imaginal discs of Ceratitis and Musca................................. 75

6.2.2 Gene expression patterns in Ceratitis and Musca embryos.......................................... 80

6.3 Discussion and future work .................................................................................... 85

6.3.1 Drosophila, Ceratitis, and Musca homologous genes.................................................. 85

6.3.2 Anteroposterior boundary formation............................................................................. 86

6.3.3 Dorsoventral boundary formation................................................................................... 88

6.3.4 Wing patterning and vein development....................................................................... 89

7 GENOME EDITING WITH THE CRISPR/CAS9 SYSTEM.......................................... 93

7.1 Labelling the Dpp gradient...................................................................................... 93

7.2 Design of constructs for genome editing in Ceratitis and Musca...................... 94

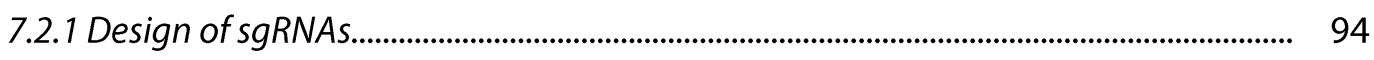

7.2.2 Design of vectors for the homology directed repair..................................................... 95

7.3 Discussion and future work............................................................................................ 96

7.3.1 Efficiency of the designed sgRNAs................................................................................... 96

7.3.2 Generation of the marker strain.......................................................................................... 96

7.3.3 Further use of the marker strain............................................................................................ 97

8 GENERAL DISCUSSION............................................................................................................ 99

8.1 Variation of size and wing shape among species.................................................. 99 
8.2 Robustness of the wing development......................................................................... 100

8.3 Amplitude of Dpp gradient in Ceratitis and Musca................................................ 102

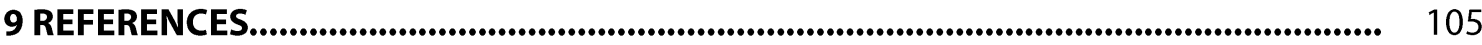

10 ACKNOWLEDGMENTS................................................................................................................ 131

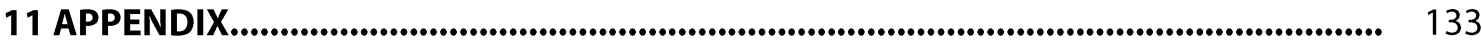

11.1 Abbreviations.................................................................................................. 133

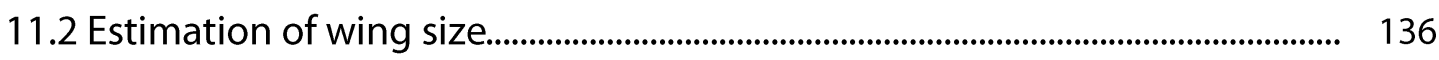

11.3 Primers used in this project........................................................................................ 137

11.4 Identification of Ceratitis and Musca genes of the wing development........ $\quad 140$

11.5 Vector maps................................................................................................................................. 162

\section{CURRICULUM VITAE}




\section{SUMMARY}

For many years, tissues growth and patterning bothered minds of scientists. Owing to numerous studies on this subject during the last 5 decades, we know that the two processes, growth and patterning, are controlled and precisely coordinated during development by small signaling molecules called morphogens. Secreted by specific cells, these molecules transfer shortand/or long-range signals to the neighboring tissue. Patterning mechanisms are well explored, while knowledge on growth control by morphogens remained incomplete. A number of different models have been proposed to explain this phenomenon in developing Drosophila wings, which is the simplest 2D model to study. The proposed models are based on the classical system of Drosophila melanogaster, and they have never been tested in other closely related systems such as other flies.

In this project, I set out for the use of two larger non-model flies: Ceratitis capitata and Musca domestica. These flies belong to the same order (Diptera), represent alternative systems to study growth, and have various genome editing tools available. In this study, I compared the three dipteran species at the organismal and genetic level. I studied changes in size of pupae and adult traits in response to different rearing temperatures and densities in males and females. I confirmed a clear sexual size dimorphism for Drosophila and showed that the dimorphism is not uniform in Ceratitis and Musca. Interestingly, I found that the size changes in response to different growth conditions were sex-dependent. Comparison of static and evolutionary allometries in the three species revealed that the response to the same environmental variable was genotype specific but had similarities between the flies. In this part of work, I also showed that size of adult traits may differ among species and the use of a single trait may result in a wrong estimation of the absolute body size, which significantly influences many aspects of insects' life such as fecundity, life span, and mating success. In the context of the project, I estimated variation in the relative wing size among the three dipteran species and computed wing loading coefficients for different rearing conditions imitating various environmental situations. I found that the relative wing size varied between species and did not depend on the average size of flies.

In addition to the size estimation, I drew my attention to the variation in wing shape between D. melanogaster, C. capitata, and M. domestica. Size and shape are two parameters that are tightly related during development. To uncouple them, I applied geometric morphometrics and studied inter- and intraspecific shape differences. I found that all three species exhibited a 


\section{2 | SUMMARY}

clear sexual wing shape dimorphism. In Ceratitis and Musca, the dimorphism stayed independent of size differences between male and female wings. In contrast, in Drosophila, a large portion of the observed shape differences between males and females could be explained by the allometric component. Additionally, I revealed shape changes that occurred in consequence of different rearing conditions applied during larval growth, temperature and density, and demonstrated that these changes were sex specific. I discussed the obtained results in the light of different mating behaviors in these flies and proposed a possible explanation of the found wing shape variation.

Further, I analyzed gene expression patterns in wing imaginal discs - small structures that are placed during embryonic development, grow and differentiate during larval and pupal stages, and finally give rise to adult fly wings. I analyzed expression of a number of genes that are involved in various wing differentiation and patterning processes. I found that expression in the wing pouch region, which further develops into the wing blade, was similar among the three species. In contrast, expression of the analyzed genes varied in other tissue of the wing imaginal disc suggesting that there are certain differences in development and patterning of structures that grow from these tissues, hinge and thorax.

Since I found that development of wings was rather conserved between Drosophila, Ceratitis, and Musca, I developed an approach that can be applied in order to generate transgenic flies. These flies can be used to estimate kinetic parameters of the Dpp morphogen gradient, which is the key player involved in regulation of growth and patterning in developing Drosophila wings. I strongly believe that comparison of these processes between the three fly species would shed some light on the mystery of the growth regulation. It will help us to understand the strong connections between growth and patterning during development and open a path to new applications. 


\section{GENERAL INTRODUCTION}

\subsection{Models to study tissue growth and patterning}

For a long time, the scientific community was concerned with a question how different tissues grow and differentiate. In living organisms, these two processes are closely related and precisely coordinated during development. These coordination and control are mainly achieved by small signaling molecules, named morphogens. They are usually secreted by certain cells and transfer signals to the neighbouring tissue in a concentration-dependent manner. Secretion of a morphogen from a restricted group of cells and non-directional spreading in the target tissue form a gradient of morphogen concentration. Different levels of the morphogen gradient can be detected by cells and interpreted as a part of spatial information (Figure 2.1). In the 1960s, this idea was evolved and resulted into the French flag model (Wolpert 1969). The model states that different cells receive different amounts of the morphogen depending of their relative position to the source. As the result, receiving cells activate certain target genes in response to different concentration thresholds of the morphogen.

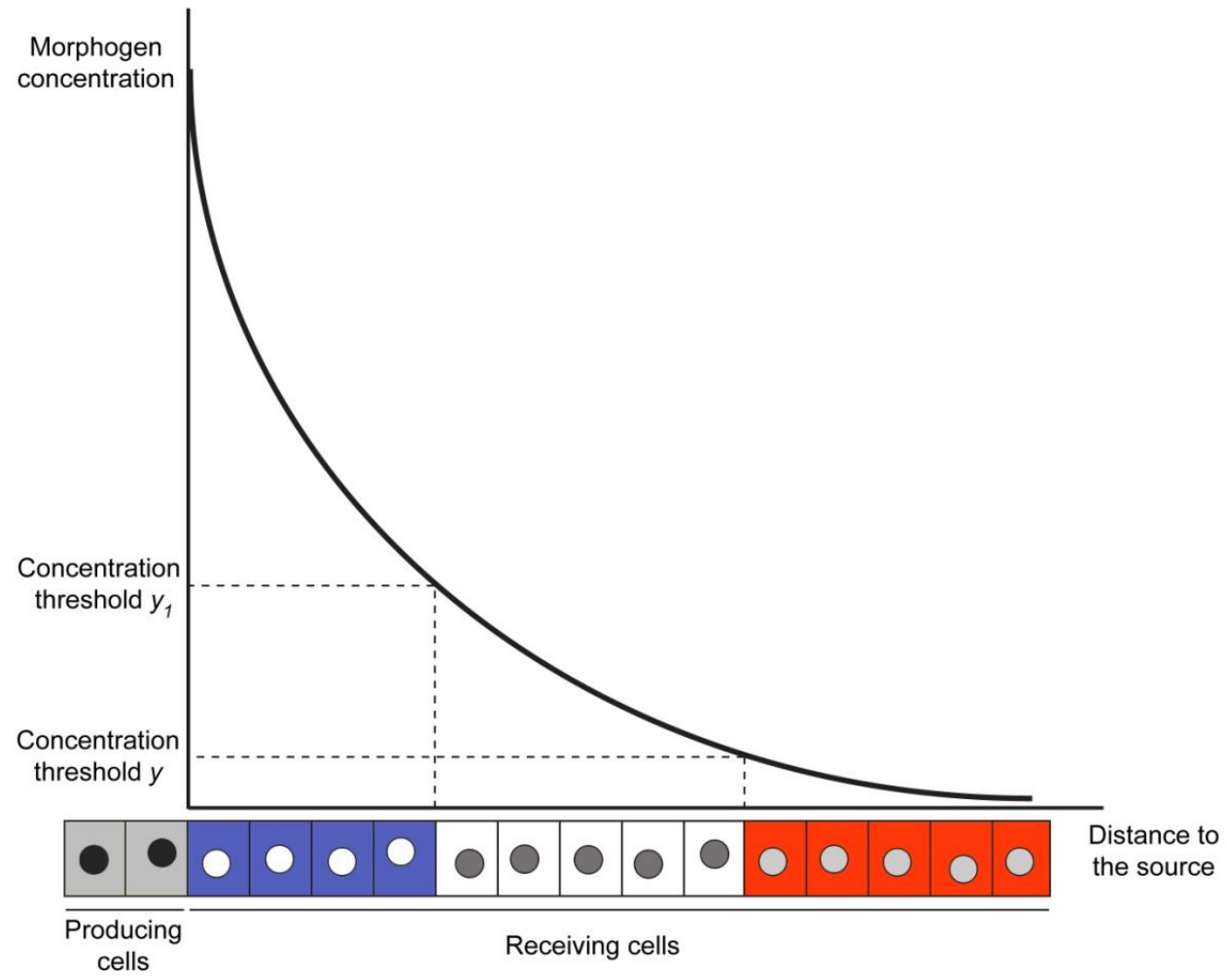

Figure 2.1: French flag model of tissue patterning by Wolpert (1969). Morphogen concentration is shown as a function of the distance to the producing cells. Cells of different colours (blue, white, and red) receive different amounts of the morphogen. Differently coloured nuclei of the receiving cells show that different target genes are activated and expressed at different threshold levels ( $y$ and $y_{1}$ ). 
Over forty years ago classical experiments confirmed that gradients of morphogens mediate pattern formation (Tickle et al. 1975; Sander 1976). Since then, this knowledge has been further developed and significantly expanded. We gained a better understanding of cell differentiation and patterning regulation via morphogens and other mechanisms (reviewed in Umulis and Othmer 2013; Restrepo et al. 2014; Heller and Fuchs 2015). In the last two decades, studies of the growth control have also focused on the signaling via morphogens. The existence of a common origin of the two processes, the growth and patterning, might explain how an organ regulates its growth until all necessary cell types are in place (Weigmann et al. 1997; Neufeld and Edgart 1998; Neufeld et al. 1998). A lot of work in this respect has been done on model systems, such as chick and mouse limb buds (Hornbruch and Wolpert 1970; Boehm et al. 2010). However, mesenchymal masses of bud cells give rise to three-dimensional structures, and this increases the complexity of the vertebrate limb system. In contrast, another model system - fly imaginal discs - simplifies the analysis of the growth regulation. Such discs appear during embryogenesis; they grow and develop during larval stages until the arrest of proliferation right before pupariation. The advantage of these structures is that the primordia of imaginal discs represent flat epithelia. This means that the identification of principles of developmental growth is reduced to a $2 \mathrm{D}$ problem, which can be solved easier.

\subsection{Dpp morphogen gradient}

An excellent model system to study the morphogenetic growth regulation in fly imaginal discs is the gradient of the morphogen Decapentaplegic (Dpp), which controls tissue growth and development in the wing disc in Drosophila melanogaster. Dpp is a bone morphogenetic protein (BMP) homologue that is involved in regulation ob both expression of different wing patterning genes and growth (reviewed in Affolter and Basler 2007). Being a morphogen, Dpp is produced in a restricted cluster of cells along the anteroposterior (AP) compartment boundary in wing imaginal discs (Figure 2.2). This strip of cells belongs to the anterior compartment which responds to the Hedgehog $(\mathrm{Hh})$ signaling coming from the posterior compartment by activation of $d p p$ expression (for details see Zecca et al. 1995). 
A
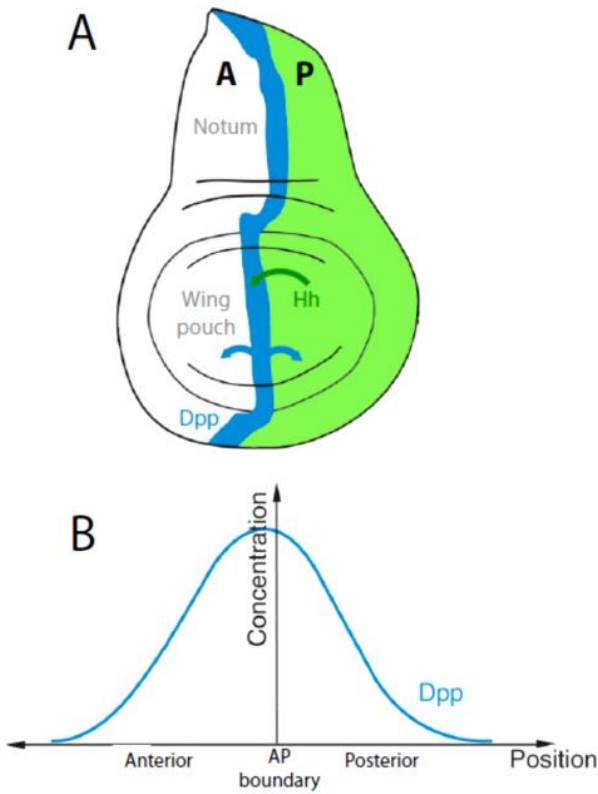

Figure 2.2: The imaginal wing disc of D. melanogaster. Expression domains of $d p p$ and $h h(\mathrm{~A})$. $\mathrm{Hh}$ is produces in the posterior $(\mathbf{P})$ compartment. It diffuses into the anterior (A) compartment and activates expression of dpp in a narrow strip of cells adjacent to the $\mathrm{P}$ compartment. Dpp spreads in the tissue and forms a gradient of concentration (B). (Modified after Schwank and Basler 2010)

When produced, Dpp is secreted from the source cells into the surrounding tissue. It spreads non-directionally and forms a gradient of concentration (Entchev et al. 2000; Tanimoto et al. 2000; Teleman and Cohen 2000; Bollenbach et al. 2008). The distribution of the gradient is balanced in two antagonistic processes: ligand diffusion and ligand degradation (Kicheva et al. 2007). Target cells receive different Dpp signaling levels through the Thickveins (Tkv) receptor and the downstream signal transduction amplifies the incoming signal in a complex non-linear way (Figure 2.3).

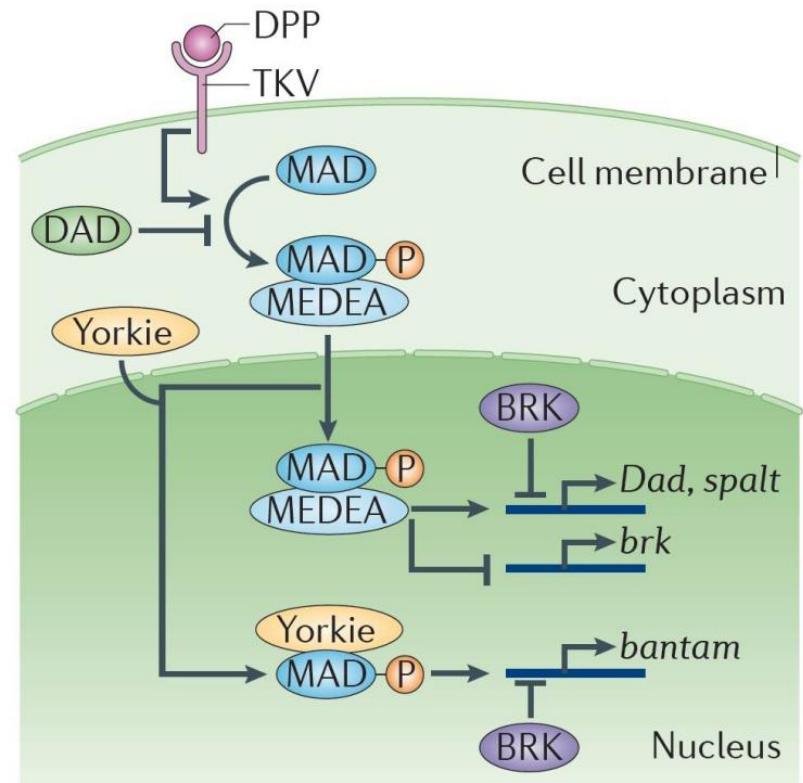

Figure 2.3: The Dpp signaling pathway. The Mad transcription factor is phosphorylated after the interaction of Dpp and the Tkv receptor. Mad can then bind Medea. It accumulates in the nucleus and represses the transcription of the repressor Brinker. Together, Brk and Mad P regulate Dpp target genes, such as daughters against Dpp (dad), spalt, and others. Dad negatively regulates Mad phosphorylation. Mad P also interacts with the co-transcriptional activator Yorkie and regulates transcription of the growth-promoting microRNA bantam. (Wartlick et al. 2011a) 


\section{6 | GENERAL INTRODUCTION}

Binding of Dpp to the receptor leads to the activation of the transcription factor Mather against Dpp (Mad) via phosphorylation (Ruberte et al. 1995; Kim et al. 1997). Mad, in its turn, activates a cascade of Dpp target genes that regulate patterning and growth of tissue in Drosophila wing imaginal discs (Figure 2.3).

\subsection{Growth model for Drosophila wing imaginal discs}

Several models have been proposed in order to explain how the Dpp gradient concentration controls the growth and division of cells in the developing wing imaginal disc (RomanovaMichaelides et al. 2015). These morphogenetic growth models can be assigned to one of the two main groups. The two groups differ by the main role that is allotted to the morphogen. One group of researchers believe that Dpp plays only a permissive role for growth, while another group holds the opinion that Dpp is instructive and that cells set their growth rates and cell cycles in accordance to the Dpp gradient property (for details see Wartlick et al. 2011a). Recent studies performed on the model of Drosophila wing imaginal discs have provided additional data proposed an explanation of growth properties and their dependence on the morphogen gradient.

In a recent paper by González-Gaitán’s group (Wartlick et al. 2011b), they quantified the Dpp concentration and signalling levels during wing disc growth. They found that both the Dpp concentration and signalling gradients scale with the tissue size and that cells of wing imaginal discs divide when Dpp signaling levels have increased by a certain amount. To obtain these results, they estimated spatial and temporal changes of the Dpp concentration, signalling activity, and disc growth parameters in developing Drosophila discs.

The Dpp morphogen gradient has a number of specific properties that quantitatively describe it (Kicheva et al. 2007; Wartlick et al. 2011b). The first quantitative parameter is the Dpp source width $(\omega)$. Variation in this parameter will necessarily result in the variation in the four key kinetic parameters, which are: (1) the production rate $(\vartheta)$; (2) effective diffusion coefficient $(D)$; (3) degradation rate $(k)$; and (4) the immobile fraction $(\psi)$. The shape of the gradient depends on the two key parameters: the concentration at the source boundary $\left(C_{0}\right)$ and decay length $(\lambda)$ that is the distance to the source where the concentration has decayed by a factor to $1 / e$ of $\mathrm{C}_{0}$. Cooperation of the mentioned above parameters results in a certain Dpp concentration at the relative position over distance or time $(C(x, t))$ with the percentage by which $\mathrm{C}_{(D p p)}$ increases during one cell cycle $(\alpha)$. All these and many other parameters were estimated in the Drosophila 
wing imaginal disc. As a result of this estimation, there was proposed a 'temporal rule' model of growth control where the cell cycle length is determined by how fast an increase of the cellular Dpp signal by 50\% is achieved (Figure 2.4) (Wartlick et al. 2011b).

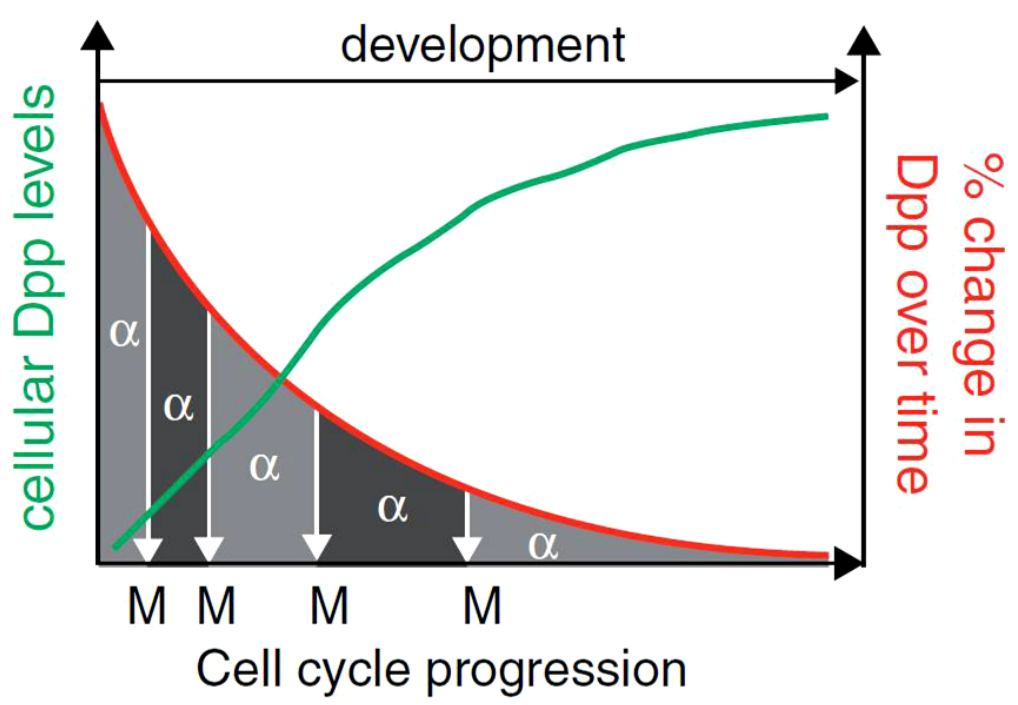

Figure. 2.4: Dpp percentage increase during development of Drosophila wing imaginal discs. Cellular Dpp levels increase during development. The percentage increase becomes smaller as absolute levels keep increasing. Autonomous cellular Dpp levels (green) for a cell and the percentage increase in Dpp levels per time unit in that cell (red) are shown. Dpp levels increase at the same relative rate for all cells. Therefore, the red line would be the same for all cells in the tissue. Onset of mitosis correlates with a threshold percentage increase $(a)$ accumulated from the beginning of the last cell cycle. (Wartlick and González-Gaitán 2011)

Indeed, this data and the model significantly expanded our understanding of the morphogen-dependent growth control, but they did not solve the problem completely. In the light of new results obtained in 2015, the role of the Dpp gradient seems to be highly controversial. Artificial elimination of the gradient in the wing disc tissue by immobilization of the enhanced GFP::Dpp on the cell surface showed that patterning and growth of central cells of the disc was negatively affected, while proliferation of lateral cells remained normal (Harmansa et al. 2015). This finding partially contradicts to the described above 'temporal rule' model and provides evidence in support to another 'growth equalization model', which suggests that Dpp in essential for division of the central cells only and has only minor affects on the lateral tissue of the Drosophila wing disc. Thus, the range of the Dpp spreading could not explain the size of the entire disc, while it could still be applicable to the wing pouch region. 


\section{8 | GENERAL INTRODUCTION}

Another recent finding has additionally increased the complexity of the Drosophila Dpp paradigm. Using a novel genome editing tool, the CRISPR/Cas9 system, it was possible to genetically remove Dpp from its endogenous strip domain in the wing disc at different developmental stages (Akiyama and Gibson 2015). This approach revealed that elimination of Dpp at early larval stages causes severe growth defects on the entire imaginal disc. In contrast, absence of the morphogen during the third larval instar results into mild defects of growth with no affect on cell proliferation. These results suggest that the Dpp gradient may include a temporal component and the importance of the morphogen may decrease during development. All these recent findings once again indicate that the growth and cell proliferation in imaginal discs have a complex regulation including different mechanisms tightly bound together. The Dpp signaling levels can be differently detected by specific cell types (lateral vs. central cells) at different developmental stages. The complexity of the described above problem still rouses hot scientific debates that become a base for novel models and future findings.

\subsection{Generalization of growth models}

The vinegar fly $D$. melanogaster is a classical model organism that is widely used as a biological system to study different fundamental processes. This is the main reason why previous studies have been focused on Drosophila wing imaginal discs and the Drosophila Dpp gradient. All gradient properties and models were elaborated on example of this species and have never been tested in closely related animals such as other insects. In this project, I set out for the use of nonmodel flies to test whether the Dpp-based mechanism can be extrapolated to other than Drosophila organisms. For this, I chose two larger flies: the Mediterranean fruit fly, Ceratitis capitata, and the common house fly, Musca domestica.

In general, all three species belong to the order Diptera. However, C. capitata was considered to be a closer relative to Drosophila in evolutionary aspect for long time. Ceratitis belongs to the family Tephritidae, which is one of two fruit fly families. The other fruit fly family is Drosophilidae. Recent studies on evolutionary relatedness broke these traditional views though. It was shown that Drosophila is closer to the Muscidae family, not the Tephritidae (Figure 2.5) (Wiegmann et al. 2011). 


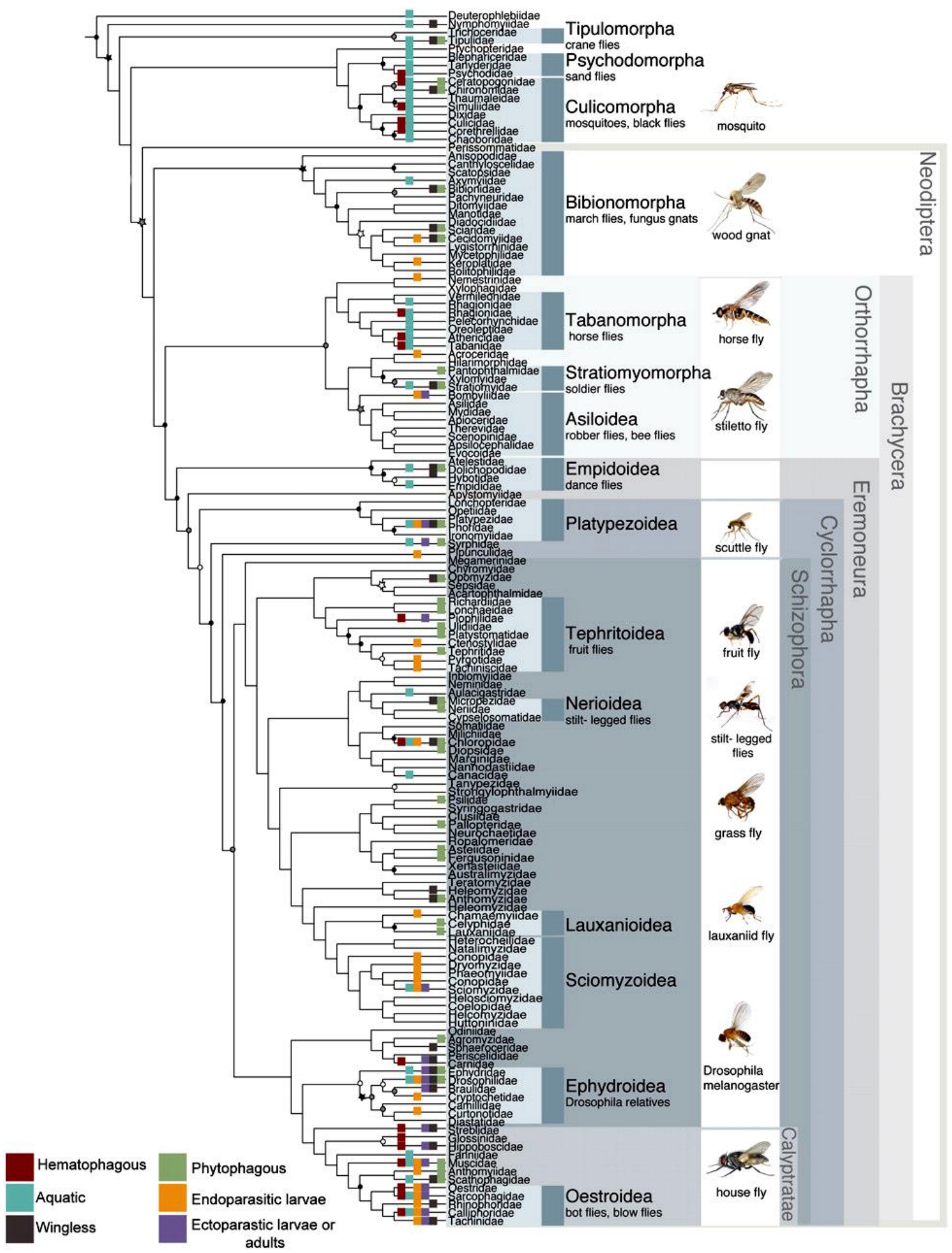

Figure 2.5: Combined molecular phylogenetic tree for Diptera. Partitioned maximum likelihood analysis calculated in RAxML. Circles indicate bootstrap support $>80 \%$. Colored squares indicate the presence, in at least one species of a family, of ecological traits mentioned to lower left. (modified after Wiegmann et al. 2011) 
These two non-model flies were chosen because in addition to the evolutionary relatedness they are of different size. As a consequence, they have different size of wing imaginal discs that develop into the largest wings in Musca, middle size in Ceratitis, and small size in Drosophila. This range of size should make it possible to determine whether quantitative parameters of the Dpp morphogen gradient in wing disks similarly influence growth and proliferation in these species. An additional advantage of using these specific non-model flies is the availability of the full genome sequence (Scott et al. 2014; Papanicolaou et al. 2016) and a number of genetic tools, such as different sets of vectors with working promoters and genes (Hediger et al. 2004; Gong et al. 2005), well-established microinjections (Handler and James 2000; Yoshiyama et al. 2000), various transgenic strains (Hediger et al. 2001; Scolari et al. 2008), etc.

Before estimating growth properties and quantifying gradient scaling, a lot of preliminary work is required to properly describe and explore the non-model species. As I previously mentioned, one of the reasons to select these specific flies was their size variation with Musca being the biggest fly and Drosophila the smallest. This description is, however, not precise enough since I do not know their relative size. In order to clarify this aspect, I inspected the size variation in the three flies (D. melanogaster, C. capitata, and M. domestica), its properties in respect to sexual dimorphism, and a possible influence on the flight performance (Chapter 4). Being a consequence of size in many respects, shape variation is another specific property of wings. Since the Dpp morphogen maintains both the growth and patterning of wing discs and regulates many genes involved in the further wing development, I analyzed inter- and intraspecific shape differences of wings in the chosen fly species (Chapter 5). To estimate similarity and robustness of the wing development, I applied environmental factors that are known to influence wing size and shape in flies and analyzed changes caused by different rearing conditions (Chapters 4 and 5). I also compared expression patterns of genes homologous between D. melanogaster, C. capitata, and $M$. domestica that play key roles in cell specification and regulate wing development (Chapter 6). Finally, I described an approach that allows quantification of the Dpp gradient in the two nonmodel species (Chapter 7). 


\section{MATERIALS AND METHODS}

\subsection{Fly stocks}

In this study, I used five strains of three different fly species listed in Table 3.1.

Table 3.1: Summary of the fly species used in the present study.

\begin{tabular}{c|cc}
\hline \multicolumn{1}{c}{ Species } & Strain & Origin \\
\hline Drosophila melanogaster & $\mathrm{w}^{1118}$ & Bloomington Drosophila Stock Center (BDSC) \\
\hline Ceratitis capitata & Egypt II & International Atomic Energy Agency (IAEA) \\
& 1247_f1m2 & $\begin{array}{c}\text { Department of Developmental Biology, } \\
\text { Georg-August-University Göttingen }\end{array}$ \\
\hline Musca domestica & ITA1 & $\begin{array}{c}\text { Altavilla Silentia, Italy 2013 (L. Beukeboom and } \\
\text { Y. Wu, GELIFES, The Netherlands) } \\
\end{array}$ \\
& M 3-6 & Institute of Molecular Life Sciences, \\
& & University of Zurich (Dr. Daniel Bopp) \\
\hline
\end{tabular}

D. melanogaster $\mathrm{w}^{1118}$ was kept at $18^{\circ} \mathrm{C}$ on food composed by $400 \mathrm{~g}$ of malt extract, $400 \mathrm{~g}$ of corn flour, $50 \mathrm{~g}$ of soy flour, $110 \mathrm{~g}$ of sugar beet syrup, $51 \mathrm{~g}$ of agar, $90 \mathrm{~g}$ of yeast extract, $31.5 \mathrm{ml}$ of propionic acid and $7.5 \mathrm{~g}$ of Nipagin dissolved in $40 \mathrm{ml}$ of Ethanol, water up to 51 . The M. domestica strains were reared at room temperature $(\mathrm{RT})\left(22 \pm 2^{\circ} \mathrm{C}\right)$ on food composed by $500 \mathrm{~g}$ of wheat bran, $75 \mathrm{~g}$ of wheat flour, $60 \mathrm{~g}$ of milk powder, $25 \mathrm{~g}$ of yeast extract, $872 \mathrm{ml}$ of water and $18.85 \mathrm{ml}$ of Nipagin (2.86 $\mathrm{g}$ of Nipagin in $10 \mathrm{ml}$ of Ethanol). Adult Musca flies were provided with sugar water only. C. capitata was kept at $28^{\circ} \mathrm{C}, 55 \pm 5 \% \mathrm{RH}$ on an artificial diet composed by $52.5 \mathrm{~g}$ of yeast extract, $52.5 \mathrm{~g}$ of carrot powder, $2 \mathrm{~g}$ of Sodium benzoate, $1.75 \mathrm{~g}$ of agar, $2.25 \mathrm{ml}$ of $32 \% \mathrm{HCl}, 5 \mathrm{ml}$ of Nipagin ( $2.86 \mathrm{~g}$ of Nipagin in $10 \mathrm{ml}$ of Ethanol), water up to $500 \mathrm{ml}$ for larvae. For adult flies, 1:3 mixture of sugar and yeast extract was used.

\subsection{Generation of differently sized flies}

All experiments on estimation of size and shape of wings in differently sized flies were performed using D. melanogaster $\mathrm{w}^{1118}$, C. capitata EgyptII, and M. domestica ITA1. To generate a range of sizes, I applied two environmental factors known to highly influence the overall body size - temperature and density. Before the experiment, Drosophila flies were placed at $25^{\circ} \mathrm{C}$ for two days. On the third day, flies were moved from vials into egg-collecting chambers and provided 
with apple-agar plates. Three hours later, apple agar plates with laid egg were collected every hour and kept at $25^{\circ} \mathrm{C}$ for $24 \mathrm{~h}$ to allow embryonic development. The freshly hatched first-instar larvae were transferred with a brush into $50 \mathrm{ml}$ vials with $15 \mathrm{ml}$ of fly food. One set of three vials with 25 larvae (low density) and three vials with 300 larvae (high density) was moved to $18^{\circ} \mathrm{C}$, while the second set of six vials with the same densities was left at $25^{\circ} \mathrm{C}$.

Ceratitis flies laid eggs through a net in water at $28^{\circ} \mathrm{C}$. Every hour, eggs were collected and transferred on larval food. $22 \mathrm{~h}$ later, first-instar larvae were transferred into small Petri dishes (diameter $\varnothing=55 \mathrm{~mm}$ ) with $15 \mathrm{ml}$ of larval food in three densities: 25 (low density), 100 (middle density), and 300 (high density) larvae per plate. One set of two plates of each density were moved to $18^{\circ} \mathrm{C}$. The second set of six plates was left at $28^{\circ} \mathrm{C}$ for further development.

Musca eggs were collected for $24 \mathrm{~h}$ in wet larval food at RT. On the day of the experiment, all hatched larvae were removed from food, and only larvae hatched within the next hour were transferred into $50 \mathrm{ml}$ vials with $5 \mathrm{~g}$ of food. Collection of larvae was repeated several times to obtain two experimental sets with three repeats of three experimental densities 10 (low density), 20 (middle density), and 40 (high density) larvae. One set of nine vials was moved to $18^{\circ} \mathrm{C}$, the other was left at RT. Ceratitis and Musca pupae were collected, photographed and kept in individual vials until eclosion. Each vial was provided with a wet sponge that was refreshed every second day.

\subsection{Estimation of size and shape parameters in Drosophila, Ceratitis, and}

\section{Musca}

\subsubsection{Photo imaging and landmark acquisition}

For every combination of environmental factors, I randomly picked at least five Musca flies of each sex and ten flies of each sex of Drosophila and Ceratitis. Selected flies were photographed from the dorsal side to capture the thorax length. The right metathoracic right leg and both wings were dissected in 2-Propanol 100\% (ROTISOLV ${ }^{\circledR}$, Carl Roth GmbH, Karlsruhe, Germany), embedded on a microscope slide in the Roti®-Histokitt II (Carl Roth GmbH, Karlsruhe, Germany), and photographed under the Leica MZ16 FA stereo microscope (Leica, Wetzlar, Germany) with the QImaging Micro Publisher 5.0 RTV Camera (Qimaging, Burnaby, Canada).

To provide an adequate coverage of the wing surface, I defined 11 landmarks on Drosophila and 13 landmarks on Ceratitis and Musca wings (Figure 3.1). The landmarks were the following 
(nomenclature is given after Colless and McAlpine, 1991 (Colless and McAlpine 1991)): 1 , branching point of veins $R_{1}$ and $R_{S}$ (base of $R_{2+3}$ and $R_{4+5}$ ); 2, branching point of veins $R_{2+3}$ and $\mathrm{R}_{4+5} ; 3$, intersection of veins $\mathrm{C}$ and $\mathrm{R}_{1} ; 4$, intersection of vein $\mathrm{R}_{4+5}$ and crossvein $r-m$ (anterior crossvein); 5 , intersection of crossvein $r-m$ and vein $\mathrm{M}_{1+2} ; 6$, intersection of vein $\mathrm{M}_{1+2}$ and crossvein $i-m$ (posterior crossvein); 7 , intersection of crossvein $i-m$ and vein $M_{3+4} ; 8$, intersection of $\mathrm{M}_{3+4}$ and the wing margin; 9, intersection of veins $C$ and $R_{2+3} ; 10$, intersection of veins $C$ and $\mathrm{R}_{4+5} ; 11$, meeting point of the anal part of the wing and the alula; 12 , intersection of veins $\mathrm{CuA}_{2}$ and $\mathrm{A}_{1}+\mathrm{CuA} \mathrm{A}_{2}$ (Ceratitis) or the tip of vein $\mathrm{A}_{2}(\mathrm{Musca})$ : 13 , intersection of $\mathrm{A}_{1}+\mathrm{CuA}_{2}$ and the wing margin (Ceratitis) or the tip of vein $\mathrm{A}_{1}+\mathrm{CuA}_{2}$ (Musca). The landmarks were digitized using tpsUtil (Rohlf, 2004) and tpsDig2 (Rohlf, 2010).

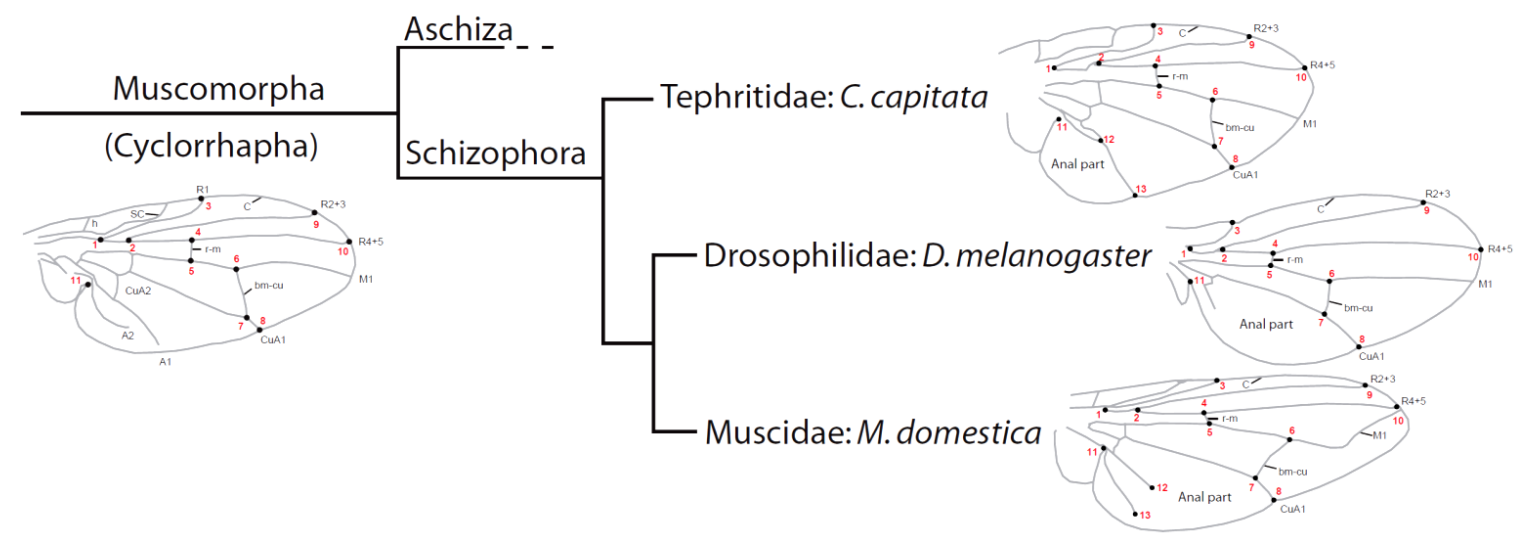

Figure 3.1: Phylogenetic tree of Muscomorpha with wing outlines and landmarks used for this study. Homologous landmarks 1 to 11 as well as additional landmarks 12 and 13 in Ceratitis and Musca are shown as black points with the respective number. The tree is base on the phylogeny by Wiegmann et al. (2011). Vein abbreviations: A - anal vein; bm-cu - basal-madial-cubital crossvein; CuA - anterior cubital vein; $\mathrm{C}$ - costal vein; $\mathrm{h}$ - humeral crossvein; $\mathrm{M}$ - medial vein; $\mathrm{R}$ - radial vein; $\mathrm{r}-\mathrm{m}$ - radio-medial crossvein; $\mathrm{SC}$ - subcosta. Branch lengths of the tree do not indicate evolutionary time or distance.

\subsubsection{Estimation of size}

\subsubsection{Measurements and computation of size estimators}

All Ceratitis and Musca pupae were weighed on the $2^{\text {nd }}$ day after pupation and left for further development and eclosion. The pupal volume was calculated with the ellipse volume equation $\mathrm{PV}=4 / 3 \pi^{\star} \mathrm{PL}^{\star}(\mathrm{PW} / 2)^{2}$, where $\mathrm{PV}$ - pupal volume, $\mathrm{PL}$ - pupal length, measured as a distance from the most apical to the most distal point of the pupa, and PW - pupal width, measured in the widest centre region of the pupae. All distances were measured with an accuracy of $\pm 5 \mu \mathrm{m}$. Images with broken or deformed during preparation samples were excluded from the analysis. Pupal size was computed as the Principal Component 1 (PC1) of the pupal weight and 
volume with the Principal Component Analysis (PCA) implemented in R (R Development Core Team 2008). Additionally, for each fly, I measured the tibia length and thorax length, defined as the distance from the anterior margin of the thorax to the posterior tip of the scutellum (Figure 3.2, top and middle).
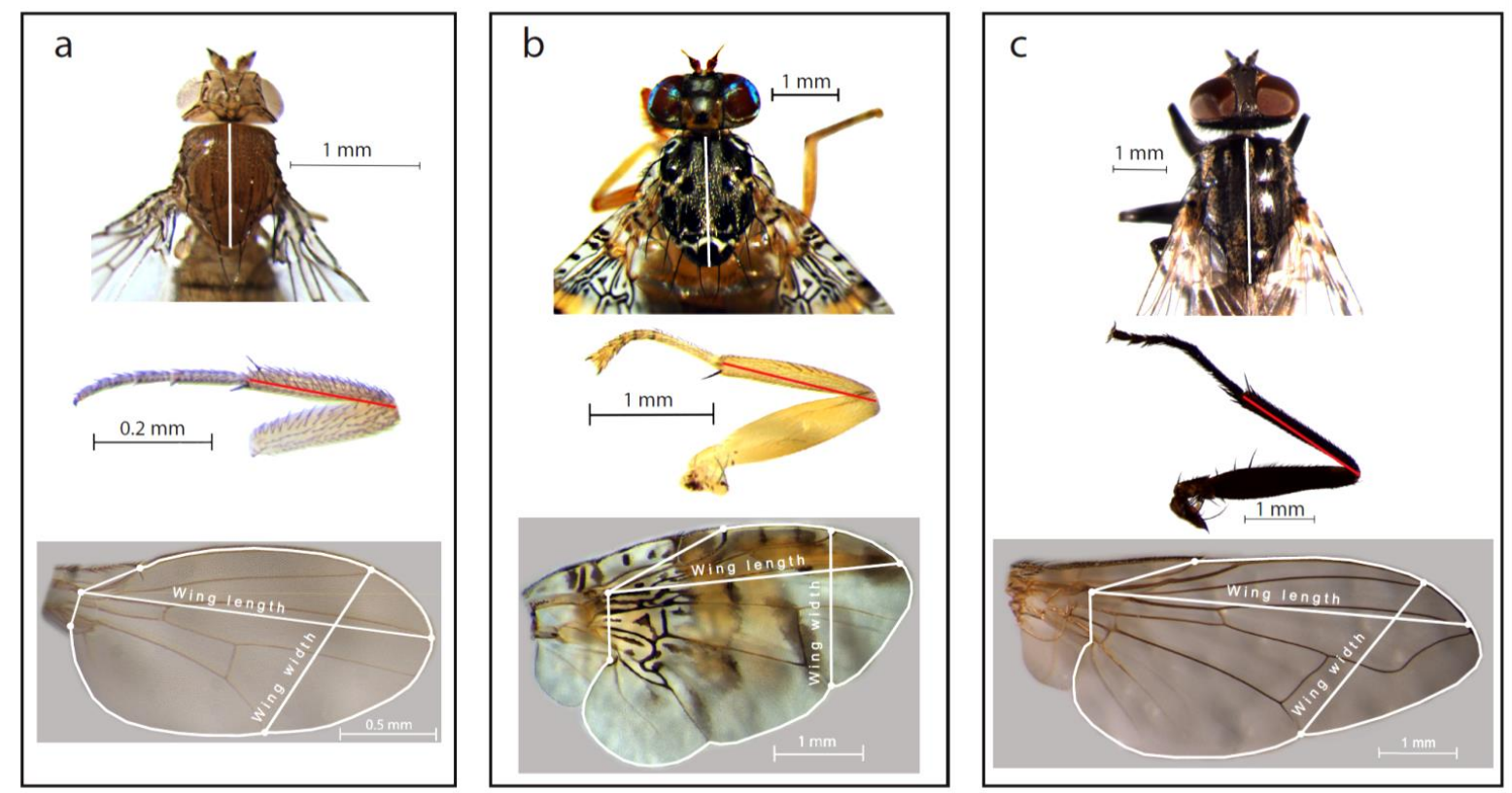

Figure 3.2: Adult traits of flies with measurements. The thorax length, tibia length, and wing measurements (i.e., the length, width, and outline for the manually measured area) in D. melanogaster (a), C. capitata (b), and M. domestica (c).

Every wing was described with three metrics obtained from row landmark coordinates: the wing length (distance from landmark 1 to landmark 10), wing width (distance from landmark 8 to landmark 9), and wing area (WA), restricted by landmarks 1, 3 and 11 (Fig. 3.2, bottom). I manually measured area for 35 wings randomly taken from different experimental groups. For the same set of wings, I computed the wing centroid size (WCS) from landmarks 1-10 for Drosophila and landmarks 1-13 for Musca and Ceratitis (Figure 3.1) as the square root of the sum of squared deviations of landmarks around their centroid (Bookstein 1996; Dryden and Mardia 1998; Slice 2005), using the MorphoJ software (Klingenberg 2008). To check correlation of the WCS and manually measured WA, I performed Spearman's rank correlation test and found a high correlation between the two parameters in Drosophila (0.993, p<0.05) and Musca $(0.992, \mathrm{p}<0.05)$ and relatively low in Ceratitis (0.54, $\mathrm{p}<0.05)$. Therefore, for Drosophila and Musca, I transformed the WCS into the WA using the deduced correlation equations (for Drosophila 
wing area $=1,348 \times \mathrm{WCS}-1,125$; for Musca wing area $=3,185 \times \mathrm{WCS}-8,674$; see Figure 4.1), while for Ceratitis I manually measured the WA using Analysis tools of Adobe Photoshop CS5. All numbers obtained for the right and left wings were averaged for each individual. If only one wing was available for a fly, it was used as the mean. Finally, for each fly, I computed the body size coefficient (BSC) as the PC1 of the thorax length, tibia length, and WA with the PCA implemented in R. When analyzing wing loading, the WA was excluded from the BSC.

\subsubsection{Computation of allometric coefficients}

Usually, scaling relationships are modelled with the allometric equation $y=a x^{b}$, where $y$ and $x$ are measurements for two given traits and $b$ is the allometric coefficient that shows relationships between the traits (Huxley 1924; Huxley and Tessier 1936). Log-transformation of the allometric equation results into a linear relationship: $\log (y)=\log (a)+b \times \log (x)$, where $b$ is a slope and $\log (a)$ is an intercept. The value of the coefficient $b$ indicates whether a given trait grows proportionally (isometrically) with the overall body size $(b=1)$, hypo- or hyperallometrically $(b<1$ and $b>1$, respectively). In this study, I computed allometric coefficients for the wing area, thorax, and tibia from the log-transformed data for all possible combinations of analyzed conditions using method described in (Shingleton et al. 2009) with the pca() function in the labdsv package in R.

\subsubsection{The relative wing size and wing loading}

To compare the relative wing size between the flies, I corrected the WA for body size using a linear regression with the BSC being the explanatory variable for each individual. Residuals of the regression were plotted for each species in R and a Mann-Whitney U-test was performed for the pair wise comparison with STATISTICA 12 (StatSoft Inc. 1997). Additionally, the wing loading coefficient for each fly was computed as described in (Starmer and Wolf 1989). For this, the cubed thorax length was divided by the WA averaged for the right and left wings. The result was additionally tested using the BSC as the measure of the absolute body size.

\subsubsection{Counting bristles on the wing membrane}

It was previously shown that one epithelial cell of the Drosophila wing produces only one bristle (Dobzhansky 1929). Therefore, I simply counted bristles to roughly estimate the cell number in wings of Drosophila and Ceratitis. For this, I took pictures of at least five right and five 
left wings in high magnification and counted bristles in the wing area along the wing width line similarly defined for flies grown at the optimal temperature $\left(25^{\circ} \mathrm{C}\right.$ for Drosophila and $28^{\circ} \mathrm{C}$ for Ceratitis) and low density. The area where bristles were counted was defined by a line crossing the intersection of the $\mathrm{C}$ and $\mathrm{R}_{2+3}$ veins on one side and the intersection of $\mathrm{CuA}_{1}$ and the wing margin one another side (see Figure 4.6 for detailes). Another parallel line was $1 \mathrm{~cm}$ aside towards the wing center. The $1 \mathrm{~cm}$ distance was measured along the $C$ vein and along the wing margin near the $\mathrm{CuA}_{1}$ vein. Number of rows of bristles was estimated in three areas: near the $\mathrm{R}_{2+3}$ vein, in between the $\mathrm{R}_{4+5}$ and $\mathrm{M}_{1}$ veins, and near the $\mathrm{CuA}_{1}$ vein. Then, an average number of bristles in a row along the wing width line was computed by dividing the number of bristles in the area by the number of rows (Appendix 11.2, Table 11.1 in there).

\subsubsection{Statistics}

Statistical significance of size changes was tested with STATISTICA 12. My data did not show the normal distribution; therefore, I used non-parametric statistical tests. Correlation between the WCS and the manually measured WA, as well as pair wise correlations between the measured body parts were estimated with Spearman's rank correlation coefficient. To test correlation of the WCS with other wing measurements, I performed a linear regression and plotted the regression lines. Effects of the rearing temperature and density on size and their significance were tested with Mann-Whitney $U$-test.

\subsubsection{Wing morphometrics}

\subsubsection{Procrustes superimposition}

In this study, wing shape was estimated using landmark-based geometric morphometric methods (Rohlf 1990; Bookstein 1991). With superimposition methods (e.g. generalized Procrustes analysis, GPA), it is possible to register landmarks of a sample to a common coordinate system in three steps: translating all landmark configurations to the same centroid, scaling the configurations to the same centroid size, and rotating the configurations until the summed squared distances between the landmarks and their corresponding sample average have the minimum scaling (Slice 2005; Mitteroecker et al. 2013).

For the collected wings I applied the GPA (Dryden and Mardia 1998; Slice 2005) in MorphoJ (Klingenberg 2008). The wings were aligned along the $\mathrm{R}_{4+5}$ vein (landmarks 1 to 10 ), the 
mean configuration of landmarks was computed, and each wing was projected to a linear shape tangent space. The coordinates of the aligned wings were the Procrustes coordinates. It has already been shown that fly wings exhibit directional asymmetry (Klingenberg et al. 1998). Because asymmetry was not of interest in this study, I averaged coordinates of the right and left wings for each individual. If only one wing was present for a fly, I used it as the mean. The obtained averaged Procrustes coordinates were used as shape variables in further analyses.

\subsubsection{Estimation of growth trajectories}

Extraction of shape from landmarks in GPA removes variation in wing size. However, the shape data obtained in such way still contain a size component, the allometric shape variation (Huxley 1924; Huxley 1932). This variation accounts for the shape changes occurred due to increase in the size of the organ. To quantify the size of fly wings, I used the WCS. To determine growth trajectories and characterize morphological changes in response to the size increase, I used MorphoJ and applied a multivariate regression of the Procrustes coordinates on the WCS pooling among sub-groups of temperature and density. The amount of shape variation was given as a percentage of the total variation around the sample mean. Percentage numbers show the relative importance of allometry for the shape variation in each species as well as in two sexes separately. I also applied a permutation test with 10000 runs (Pitman 1937; Good 1994) to test independence between the size and shape changes. Additionally, I computed shape scores according to Drake and Klingenberg (2008). These shape scores are the shape variables associated with the shape changes predicted by the regression model. It also includes the residual variation in shape space in the size direction. To visualize the association between size and shape, I plotted the shape scores against the WCS. Similarity between trajectories was estimated using the Analysis of Covariance (ANCOVA) in R software (aov() package) with the WCS as an explanatory variable.

\subsubsection{Interspecies comparison}

To compare the wing shape variation among species, I created a new dataset with all wings polled together. All shape comparisons and permutation tests were carried in the MorphoJ software. To identify and remove the allometric component of the shape variation, I applied a multivariate regression. For the regression, I used the WCS computed from the homologous

landmarks, 1 to 11 for each species. Subsequently, I performed a PCA to visualize the 
non-allometric component of shape in a scatter plot and visualized morphological differences by thin-plate spline (TPS) deformation grids (Thompson 1917; Bookstein 1991; Rohlf and Marcus 1993; Slice 2005). Magnitudes of shape differences between fly wings were computed with the canonical variate analysis (CVA) and expressed in units of Procrustes distance, which is the square root of the sum of squared distances between corresponding landmarks. Significance of the results was tested with permutation tests with 10000 runs.

\subsubsection{Intraspecies comparison: sexual dimorphism, temperature and density effects}

The comparison of different shape forms within species was performed using 11 landmarks in Drosophila and 13 landmarks in Ceratitis and Musca. First, I tested whether there were effects of the sex, rearing temperature, and density. I run the Procrustes ANOVA test in MorphoJ, found clear effects of each of the parameters, and therefore continued with the more detailed shape analysis.

Sexual shape dimorphism (SShD) was estimated for the allometric and non-allometric components of the shape variation together (total SShD) as well as for the pure shape only (nonallometric component). For each species I performed a size correction by the allometric regression and used residuals for later analysis. Magnitudes of SShD were estimated with the discriminant function analysis (DFA) and expressed in units of Procrustes distance. DFA identifies shape features that have the most difference between groups relative to within group variation, and this test can only be applicable to two experimental groups. Therefore, I used the DFA to define SShD, effects of the rearing temperature (high and low) in each species, and density effect in the two Drosophila groups. To better visualize wing changes, I used species specific warped outline drawings with a different scale factor mentioned in figure legends. I also provided discriminant scores for each DFA. To estimate shape changes originated from different rearing densities in Ceratitis and Musca, I applied a CVA, designed to look for a variation among three or more groups. In addition to the CVA, I run a DFA for the two groups representing the density extremes (the highest and the lowest number of larvae per plate/vial). For each test, I run a permutation test with 10,000 random permutations to show significance of the results. 


\subsection{Molecular biology}

\subsubsection{Oligonucleotides}

Gene specific primers for Ceratitis and Musca were designed based on the genome and transcriptome sequences available at the National Center for Biotechnology Information (NCBI, http://www.ncbi.nlm.nih.gov/). Primers were generated either manually or using Primer-BLAST, which is a tool designed for finding specific primers and available at http://www.ncbi.nlm.nih.gov/tools/primer-blast/. Oligonucleotides were synthesized by Eurofins MWG Operon (Ebersberg, Germany). A complete list of primers with their sequences and respective genes can be found in Appendix 11.3.

\subsubsection{Isolation of mRNA and synthesis of CDNA}

For further experiments, mRNA was isolated from 0-48 h old Ceratitis and Musca embryos as well as Ceratitis wing imaginal discs dissected from the larval stage L3 using the ZR Tissue \& Insect RNA MicroPrep ${ }^{\text {tw }}$ (Zymo Research, Irvine, USA). Quality of mRNA was checked with the agarose gel electrophoresis and the concentration was estimated with a NanoDrop ${ }^{\circ}$ spectrophotometer (ND-1000, software V3.7.1, Thermo Fisher Scientific Germany BV \& Co KG, Braunschweig, Germany). Freshly isolated mRNA was further used for cDNA synthesis with SMART PCR cDNA Synthesis kit (Clontech, Saint-Germain-en-Laye, France). Both mRNA and cDNA were stored in aliquots at $-80^{\circ} \mathrm{C}$ until required.

\subsubsection{Rapid amplification of CDNA ends}

The 5'- and 3'-RACE-ready cDNA libraries were synthesized from embryonic poly(A) mRNA extracted from Ceratitis and Musca. To perform rapid amplification of cDNA ends (RACE-PCR), the SMART ${ }^{\mathrm{TM}}$ RACE cDNA Amplification Kit (Clontech, Saint-Germain-en-Laye, France) and the Advantage 2 PCR Kit (Clontech, Saint-Germain-en-Laye, France) were used according to the protocol by the manufacturer.

\subsubsection{Cloning}

Molecular cloning was performed according to standard protocols (Green and Sambrook 2012) or respective user manuals. DNA sequences were amplified by PCR with respective primers and either Phusion ${ }^{\odot}$ High-Fidelity DNA polymerase (Finnzymes, Thermo Fisher Scientific 
Germany BV \& Co KG, Braunschweig, Germany) or Advantage 2 PCR Kit (Clontech, Saint-Germain-en-Laye, France) using the Eppendorf Mastercycler personal (Eppendorf AG, Hamburg, Germany). Obtained fragments were purified from agarose gel via the NucleoSpin ${ }^{\oplus}$ Gel and PCR Clean-up Kit (Macherey-Nagel, Düren, Germany), and ligated by T4 DNA ligase (Fermentas GmbH, St. Leon-Rot, Germany, and New England Biolabs, Frankfurt a.M., Germany) into pCR ${ }^{\oplus} I$ vector (the TA Cloning ${ }^{\circledR}$ Kit Dual Promoter, Invitrogen/Life Technologies GmbH, Karlsruhe, Germany).

\subsubsection{Transformation and plasmid DNA preparation}

Heat shock transformation was performed using chemically competent Escherichia coli DH5a bacteria and the respective vector. Positive colonies were selected with the blue-white selection on LB-Agar plates containing 40 $\mu \mathrm{l} \mathrm{X-Gal} \mathrm{(4 \%} \mathrm{in} \mathrm{DMF).} \mathrm{Plasmid} \mathrm{DNA} \mathrm{from} \mathrm{the} \mathrm{positive}$ clones was isolated with a modified alkaline cell lysis protocol (Pechmann 2011) or with the NucleoSpin ${ }^{\circ}$ Plasmid Miniprep Kit (Macharey-Nagel, Duren, Germany) and tested by the restriction digest with EcoRV restriction enzyme (New England Biolabs, Ipswich, MA, USA) in case of pCRII vector or with EcoRI restriction enzyme (New England Biolabs, Ipswich, MA, USA) in case of pJET1.2/blunt vector. Selected plasmids were sent for sequencing (LGC Genomics, Berlin, Germany).

\subsection{Localization of gene expression in fly embryos and wing imaginal discs}

\subsubsection{Preparation and storage of embryos and larval tissue}

Ceratitis and Musca embryos of different stages were collected and fixed in 1:1 mixture of 4\% paraformaldehyde (PFA, SIGMA-ALDRICH ${ }^{\circ}$ Chemie GmbH, Munich, Germany) and n-Heptane (ROTIPURAN ${ }^{\circledR}$ SUPRA, Carl Roth GmbH, Karlsruhe, Germany), the vitelline membrane was removed and the embryos were stored in methanol 100\% (SupraSolv ${ }^{\oplus}$ ECD and FID, Merck Millipore KGaA, Darmstadt, Germany) at $-20^{\circ} \mathrm{C}$. For dissections of wing imaginal discs, L3 instars were collected. Staging of larvae was performed by both total incubation time and the optical examination of behavior specific for the pre-pupal stage. Dissections were carried out in phosphate-buffered saline (PBS, 10x stock: $1.37 \mathrm{M} \mathrm{NaCl}, 27 \mathrm{mM} \mathrm{KCl}, 20 \mathrm{mM} \mathrm{KH} 2 \mathrm{PO} 4$, $100 \mathrm{mM} \mathrm{Na2HPO} 4, \mathrm{pH}$ 7.4) on ice. The mouth parts of the selected larva were grasped with tweezers and one third of the abdomen was cut off with scissors. The larva was everted, wing 
imaginal discs were removed with another pair of tweezers and fixed in 4\% PFA for 30 min. After fixation, discs were washed with PBST (1x PBS with $0.1 \%$ TritonX-100) two times, dehydrated with methanol $100 \%$, and stored at $-20^{\circ} \mathrm{C}$.

\subsubsection{Synthesis of digoxigenin-labeled RNA probes}

Antisense RNA probes were synthesized from purified PCR products containing SP6- and T7-RNA Polymerase promoter sites by using the digoxigenin (DIG) RNA Labeling Mix, SP6- or T7-RNA Polymerases, and Protector RNase Inhibitor (Roche Applied Science, Mannheim, Germany) in the in vitro transcription reaction according to the protocol by the manufacturer. Decision on what RNA Polymerase had to be used was made in accordance with the orientation of the insert. The DIG-labeled RNA probes were dissolved in deionized water and fragmented to an average length $\approx 200 \mathrm{bp}$ by adding an equal amount of sodium carbonate buffer $\left(80 \mathrm{mM} \mathrm{NaHCO}_{3}, 120 \mathrm{mM} \mathrm{Na}_{2} \mathrm{CO}_{3}, \mathrm{pH}\right.$ 10.2) and incubating at $60^{\circ} \mathrm{C}$ (Angerer and Angerer 1992). Fragmented probes were stored at $-20^{\circ} \mathrm{C}$ in a HybeA buffer ( $50 \%$ formamide, $0.1 \mu \mathrm{g} / \mu \mathrm{l}$ sonicated salmon sperm DNA, $50 \mu \mathrm{g} / \mathrm{ml}$ Heparin, $5 \mathrm{x}$ SSC and $0.1 \%$ Triton X-100, in PBS).

\subsubsection{In situ hybridization}

Fixed embryos and/or wing imaginal discs were rehydrated by rinsing them with 1:1 mixture of methanol $100 \%$ and PBT (PBS with $0.03 \%$ TritonX-100), two times with PBT, and fixed in 4\% PFA/PBT (1:1) for 20 min. After fixation, samples were washed three times with PBT for 20 min, rinsed once with HybeA/PBT mixture (1:1) and quickly washed three times with HybeA. Pre-hybridization was carried in HybeA at $65^{\circ} \mathrm{C}$ for $1 \mathrm{~h}$ minimum. After this, preheated and chilled down probes were added to samples and incubated overnight at $65^{\circ} \mathrm{C}$. On the next day, probes were discarded, samples were washed three times with HybeA at $65^{\circ} \mathrm{C}$ for $20 \mathrm{~min}$, rinsed once with HybeA/PBT (1:1) and incubated with $1 \mathrm{ml}$ of anti-DIG-AP antibody (Anti-Digoxigenin-AP, Fab fragments, Roche Applied Science, Mannheim, Germany, diluted 1:2000 in PBT) at RT. One hour later, the antibodies were removed, samples were washed three times with PBT for 20 min and three times with a freshly prepared detection NBT buffer (100 mM Tris- $\mathrm{HCl}$ with $\mathrm{pH} 9.5,100 \mathrm{mM} \mathrm{NaCl}, 50 \mathrm{mM} \mathrm{MgCl}_{2}, 0.1 \%$ TritonX-100, in water) for $5 \mathrm{~min}$. All steps preceding the staining reaction were performed in the $1.5 \mathrm{ml}$ Eppendorf tubes with many embryos or discs (at least ten). After the last washing step, samples were transferred into glass 
wells and the detection buffer was replaced with the staining solution (1 ml NBT buffer + 4.5 $\mu$ l NBT (Nitrotetrazolium Blue chloride, Carl Roth GmbH \& Co KG, Karlsruhe, Germany, $50 \mathrm{mg} / \mathrm{ml}$ in $70 \% \mathrm{DMF})+3.5 \mu \mathrm{l} \mathrm{BCIP}^{\star}$ (5-Bromo-4-chloro-3-indolyl phosphate disodium salt, SIGMA-ALDRICH ${ }^{\oplus}$ Chemie GmbH, Munich, Germany, $50 \mathrm{mg} / \mathrm{ml}$ in 100\% DMF)). Samples were incubated in the dark at RT until the staining appeared (for $15 \mathrm{~h}$ maximum). The staining reaction was stopped by washing samples three times with PBT for 10 min each. Stained samples were transferred onto a microscope slide, covered with a drop of glycerol 100\% (Glycerin, Serva, Heidelberg, Germany) and a cover slip, and inspected under a microscope.

\subsubsection{Cryosectioning}

After in situ hybridization (ISH), samples were additionally fixed for $20 \mathrm{~min}$ in 4\% PFA and washed three times in PBT for 10 min each. The fixed tissue was put into a silicon form, covered with a tissue freezing medium (Tissue-Tek ${ }^{\oplus}$ O.C.T. Compound, Science Services GmbH, München, Germany), and frozen at $-20^{\circ} \mathrm{C}$ for $10 \mathrm{~min}$. Completely frozen samples were transferred in a cryotome (Cryostat CM 1950, Leica, Nussloch, Germany) and sliced with thickness $30 \mu \mathrm{m}$. Slices were collected with a cold brash in a cold Eppendorf tube and washed with $1 \mathrm{ml}$ of PBS to melt the tissue freezing medium. In $5 \mathrm{~min}$, the melted freezing medium was removed together with PBS, samples were rinsed twice with PBS and transferred onto a microscope slide. Each sample was covered with a drop of glycerol $100 \%$, covered with a cover slip, and inspected under a microscope.

\subsection{Design of CRISPR/Cas9 system in Ceratitis and Musca}

\subsubsection{Single guide RNA}

A novel tool of in vivo genome editing, the CRISPR/Cas9 system, is based on a double cut of genomic DNA in a specific region and a further repair of the region (Ran et al. 2013). The double strand break is introduced by Cas9 restriction enzyme guided by a single guide RNA ( $\operatorname{sgRNA}$ ) that is specifically designed to the target sequence. Using Geneious ${ }^{\circledast}$ software version 8.1.5 (Kearse et al. 2012), I designed six guide RNAs (gRNAs): three for Ceratitis and three for Musca genome editing. gRNAs targeting Ceratitis dpp were ordered as oligonucleotides (see Chapter 3: Materials and Methods, Section 3.4.1) with added T7-RNA Polymerase promoter site at the 5 ' end and additional overhangs on both sides that were sticky ends (Appendix 11.3, 
Table 11.6 in there). The oligonucleotides were annealed at $97^{\circ} \mathrm{C}$ for $7 \mathrm{~min}$, slowly cooled down, and cloned into HM034-T7-BbsI-ch (Appendix 11.5) cut by BbsI restriction enzyme (New England Biolabs, Frankfurt a.M., Germany). In case of $M u s c a$, gRNAs were ordered as a part of a long primer containing T7-RNA Polymerase promoter site and a short primer sequence annealing to the HM034-T7-BbsI-ch vector (Appendix 11.3, Table 11.6 in there). The chimeric sgRNAs, including CRISPR RNA and trans-activating crRNA, were amplified with the gRNA specific primer and the reverse MK152 primer (designed by Mohammad Karami Nejad Ranjbar (Georg-August University, Göttingen)) and transcribed with the MEGAscript ${ }^{\oplus}$ T7 Kit from Ambion ${ }^{\oplus}$ (Life Technologies GmbH, Darmstadt, Germany).

\subsubsection{Design of vectors for the homologous recombination}

Using specific primers (Appendix 11.3, Table 11.5 in there), I amplified homology arms from Musca embryonic gDNA generated and provided by Yanli Wu (University of Groningen, the Netherlands and Georg-August University, Göttingen). The construct attP-3xP3-ECFP-poly(A)-attP was amplified with the attP-F/R primer provided by Hassan Mutasim Mohammed Ahmed (Georg-August University, Göttingen) from the pCRII-Ebony-3xP3 vector and cloned into pSLfa1180fa (Appendix 11.5) after its digestion with HpaI restriction enzyme (New England Biolabs, Frankfurt a.M., Germany). The left (upstream) homology arm was cloned 33bp upstream the reporter gene in the StuI restriction site (Appendix 11.5, vector pSLfa1180fa-Md:HAL-3xP3-ECFP). The second vector, a template for the homology direct repair in Ceratitis, was also based on the plasmid pSLfa1180fa. I successfully cloned the construct ECFP-poly(A)-attP in the HpaI restriction site (Appendix 11.5, vector pSLfa1180fa-Cc:ECFP). 
24 | MATERIALS AND METHODS 


\section{BODY SIZE AS AN INTEGRAL FEATURE}

Body size is an important and sometimes the crucial feature that influences many aspects of animal life such as fecundity (Nunney and Cheung 1997), life span (Khazaeli et al. 2005), and even mating success (Partridge et al.1987). In this chapter and the corresponding discussion, I examine the size variation in three different flies (D. melanogaster, C. capitata, and M. domestica), its properties in respect to sexual dimorphism, and a possible influence on the flight performance. The text, figures and figure legends are taken from (Siomava et al. 2016). (With kind permission by Springer: Development Genes and Evolution, Size relationships of different body parts in the three dipteran species Drosophila melanogaster, Ceratitis capitata, and Musca domestica (2016), Volume 226, Issue 3, pp 245-256, DOI 10.1007/s00427-016-0543-6. Authors: Natalia Siomava (contribution: experimental data, text), Ernst A Wimmer (contribution: text), and Nico Posnien (contribution: text)).

\subsection{Basis and importance of the size variation}

Body size is an important characteristic of an animal that determines not only the ecological niche the animal occupies, but also its life style and success rate. It was previously observed that smaller-sized populations of a species reside in warmer areas, while larger-sized populations are found in colder regions. In 1847, Bergmann investigated this phenomenon and stated that this rule holds true for most living organisms (Bergmann 1847). The rule was also confirmed for most poikilotherms, including insects such as different Drosophila species (Ray 1960; Atkinson 1994; Kingsolver and Huey 2008). In Drosophila various life history traits have been shown to be body size dependent. For instance, mating success of males depends on the body and wing size (Partridge et al. 1987), while female body size highly correlates with fecundity (Nunney and Cheung 1997). Even lifespan is tightly tuned with the absolute body size of Drosophila (Miller and Thomas 1958; Khazaeli et al. 2005).

The genotype alone cannot entirely explain the wide variation of the size reaction to environmental conditions observed in Drosophila. Many environmental factors play a significant role in the body size regulation influencing both the overall body size and the size of certain organs. In natural populations, increasing latitudes and altitudes have an effect similar to those of temperature (Anderson 1966; Robinson and Partridge 2001). Nutrition (Beadle et al. 1938), crowding (Santos et al. 1994), infections (DiAngelo et al. 2009) and different oxygen levels 
(Peck and Maddrell 2005) are also known to interact with fly development and regulate body size. Many of these effects are already well characterized in the classical model system D. melanogaster (Edgar 2006). On the molecular level, recent research in Drosophila revealed major gene regulatory networks and regulation mechanisms underlying the body and organ size control (Mirth and Shingleton 2012). Many of the studied pathways interact with the hormonal regulation during the larval development. For instance both insulin and ecdysone signaling pathways were found to be involved in the growth rate and nutritional reaction norms in insects (Edgar 2006; Koyama 2014; Mirth et al. 2014; Gokhale and Shingleton 2015).

Different adult organs develop from different anlagen in a close cooperation and result into certain proportions of body parts in the adult fly (Oliveira 2014). Due to the special development of holometabolous insects, interactions of the environment and a growing individual mainly occur during the feeding larval and developing pupal stages. On the other hand, the solid pupal case comprises the whole body and its volume does not depend on the environment. Thus, pupae volume is considered the best estimator of the overall body size in Drosophila (Shingleton 2008; Stillwell 2011), but this parameter is usually impossible to assess in wild populations. Thus, many researchers tend to use adult structures, such as the thorax and tibia lengths or wing size, to estimate the overall body size (Cavicchi 1989; Pitnick and Markow 1995; de Moed 1997; Kacmarczyk and Craddock 2000). While these traits are generally accepted as estimators of the absolute body size in Drosophila, it is not yet clear whether they are suitable for other dipteran species.

\subsection{Variation of size parameters in different fly species}

In this section, I present size of pupae and adult traits in three dipteran species that exhibit clear size differences: D. melanogaster, C. capitata, and M. domestica. I also estimated influence of different environmental conditions, such as temperature and larval density during growth and pupation on size of the traits and overall body size in these flies.

\subsubsection{Correlation of wing parameters}

In a number of studies, wing centroid size (WCS) or different linear parameters of wings are used as a measure of the overall wing size or the whole specimen (Klingenberg et al. 1998; Schachter-Broide et al. 2009; Rodríguez-Mendoza 2011; Prudhomme et al. 2012). Since my 
experiments include non-model organisms (C. capitata and $M$. domestica) and it is difficult to judge whether the correlation stays good in these species, I first studied the variation of wing parameters in Ceratitis and Musca and compared it to Drosophila. I found that the WCS was positively correlated with other wing parameters (wing length, width, and area) and the relationships were linear for both sexes in all species (Figure 4.1).
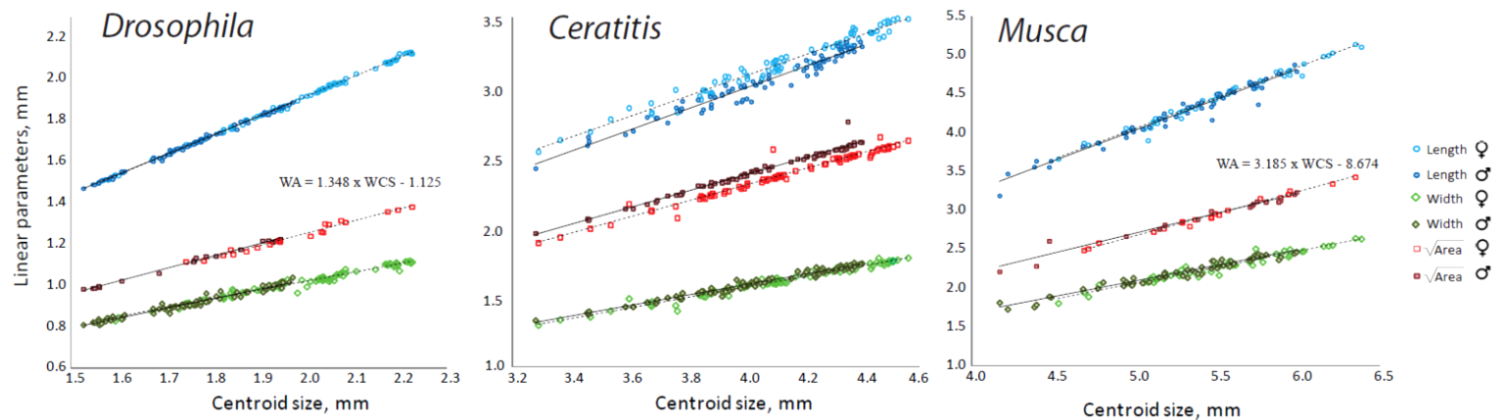

Figure 4.1: Correlation of wing parameters with the WCS in Drosophila, Ceratitis, and Musca males and females. Linear regression of wing length (blue circles), width (red squares) and square root of WA (green diamonds) on the WCS with regression lines for males (solid line) and females (dashed line) separately. The correlation equations used for the transformation of the WCS into WA values are depicted on the Drosophila and Musca plots.

Then, I tested whether the WCS can be used instead of the WA to estimate the overall wing size in D. melanogaster, C. capitata, and M. domestica. For this, I checked the correlation between these two parameters and found that WCS is well correlated with the WA and stayed independent on experimental conditions mimicking different environmental situations in two of these species, Drosophila $(0.993, \mathrm{p}<0.05)$ and Musca $(0.992, \mathrm{p}<0.05)$. However, the correlation between the two parameters was not uniform in Ceratitis (Table 4.1). It varied from 0.44 to 0.88 depending on the experimental setup and from 0.71 to 0.99 depending on sex.

Table 4.1: Correlation coefficients between the WA and WCS in C. capitata males and females in different rearing condition. Low, Mid and High corresponds to the low, middle and high densities of larvae.

\begin{tabular}{|c|c|c|c|}
\hline Density & $18^{\circ} \mathrm{C}$ & $28^{\circ} \mathrm{C}$ & \\
\hline Low & 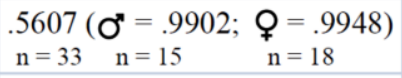 & 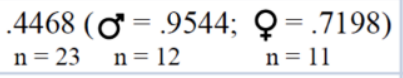 & .8448 \\
\hline Mid & $\begin{array}{llrl}.6684\left(\sigma^{\prime \prime}\right. & =.9460 ; & \text { O } & =.9091) \\
\mathrm{n}=28 \mathrm{n} & =17 & \mathrm{n} & =11\end{array}$ & $\begin{array}{lll}.6314\left(\sigma^{n}=.9422 ;\right. & Q & =.9977) \\
n=23 \quad n=12 & n & =11\end{array}$ & .9085 \\
\hline \multirow[t]{2}{*}{ High } & $\begin{array}{ll}.8790\left(\sigma^{n}=.9821 ;\right. & \stackrel{O}{+}=.9780) \\
\mathrm{n}=27 \quad \mathrm{n}=13 & \mathrm{n}=14\end{array}$ & 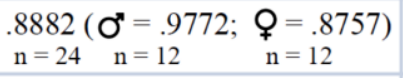 & .9560 \\
\hline & .8203 & .9144 & \\
\hline
\end{tabular}


These results demonstrate that the WCS, indeed, reflects the absolute wing size in some cases, but not always though. For example, in my experiment, I had groups with a relatively low sample of eleven individuals, but this sample size was big enough to find a good correlation, 0.997 (females, $28^{\circ} \mathrm{C}$, middle density). On the other hand, I had groups of 33 flies with a bad correlation of the WCS and WA (0.56). In this case, the reason of such low correlation was not the sample number, but rather its quality. Increasing of the sample size in situations when the correlation is relatively low will not considerably improve it. Only a wide selection of individuals from different rearing conditions can raise the coefficient to a value that is high enough to be representative.

\subsubsection{Sexual size dimorphism}

In this work, I focused on the comparison of size differences of traits known to represent body size in flies. It was already shown that D. melanogaster exhibit a clear sexual dimorphism for various body parts (Badyaev 2002; Stillwell et al. 2010). Therefore, I tested whether this trend holds true in the other two species of our research. I combined all measurements across rearing conditions and tested whether Ceratitis and Musca vary in size between males and females. I also estimated the size variation in Drosophila and compared it to the known trends in this species.

Similar to other researchers, I found a clear sexual size dimorphism (SSD) in Drosophila with females being significantly larger than males for all compared variables. In Ceratitis, I did not find any difference in the thorax length and WA, while pupae were larger and tibiae were shorter in females. Similar to Ceratitis, I found significantly longer tibiae in males of Musca. Additionally, Musca females had larger wings compared to males, while I did not detect any difference in the size of pupae and thorax length (Figure 4.2). With these results, I confirmed a clear SSD of the pupae size and body parts for Drosophila. In contrast, Ceratitis and Musca did not exhibit such a uniform sexual dimorphism in my survey. 

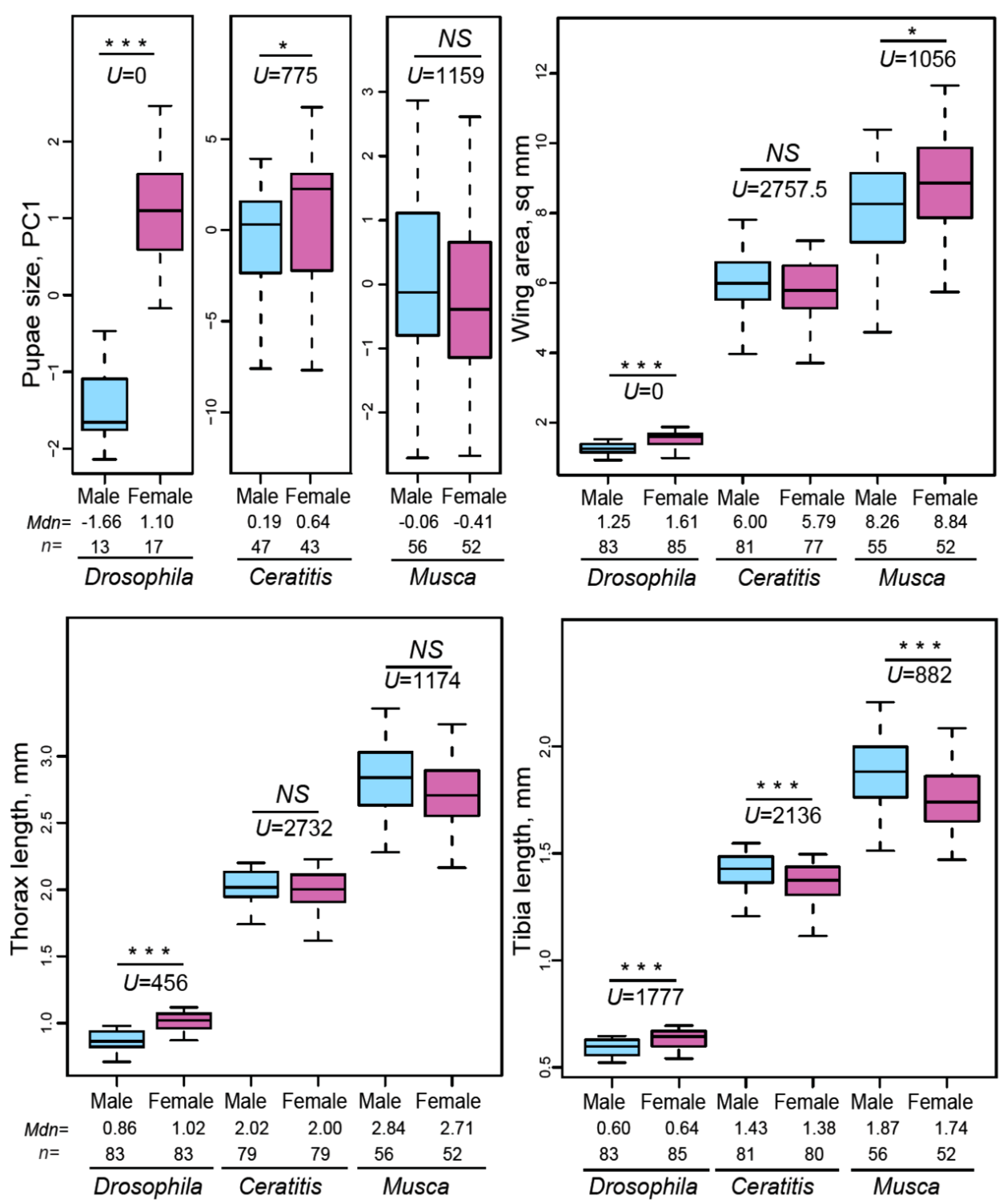

Figure 4.2: SSD in pupae and different traits. Statistical significance of difference was checked with Mann-Whitney $U$-test and shown as NS - non-significant at $p=0.05,{ }^{*}=p<0.05 ;{ }^{* *}=p<0.005 ;{ }^{* *}=p<0.0001$. $M d n$ refers to the Median of each group.

\subsubsection{Alterations of size in response to environmental cues}

In the next step, I tested whether different rearing conditions, i.e. temperature and larval density, influence size of the measured body parts and pupae in D. melanogaster, C. capitata, and M. domestica.

Comparing different rearing temperatures, I found that Ceratitis flies raised at high temperature were smaller in all measured parameters, while in Drosophila significant effects of temperature were present only for wing size. In Musca, the response was the opposite in the case of the thorax and tibia lengths, while the pupal size and WA remained unaffected by temperature 
(Figure 4.3a). Varying larval densities resulted in a steady and identical response in the three species. All adult body parts as well as the pupal size were smaller in crowded conditions, while low density resulted in bigger flies. The observed size difference between the density extremes was statistically significant for each species (Figure $4.3 \mathrm{~b}$ ).
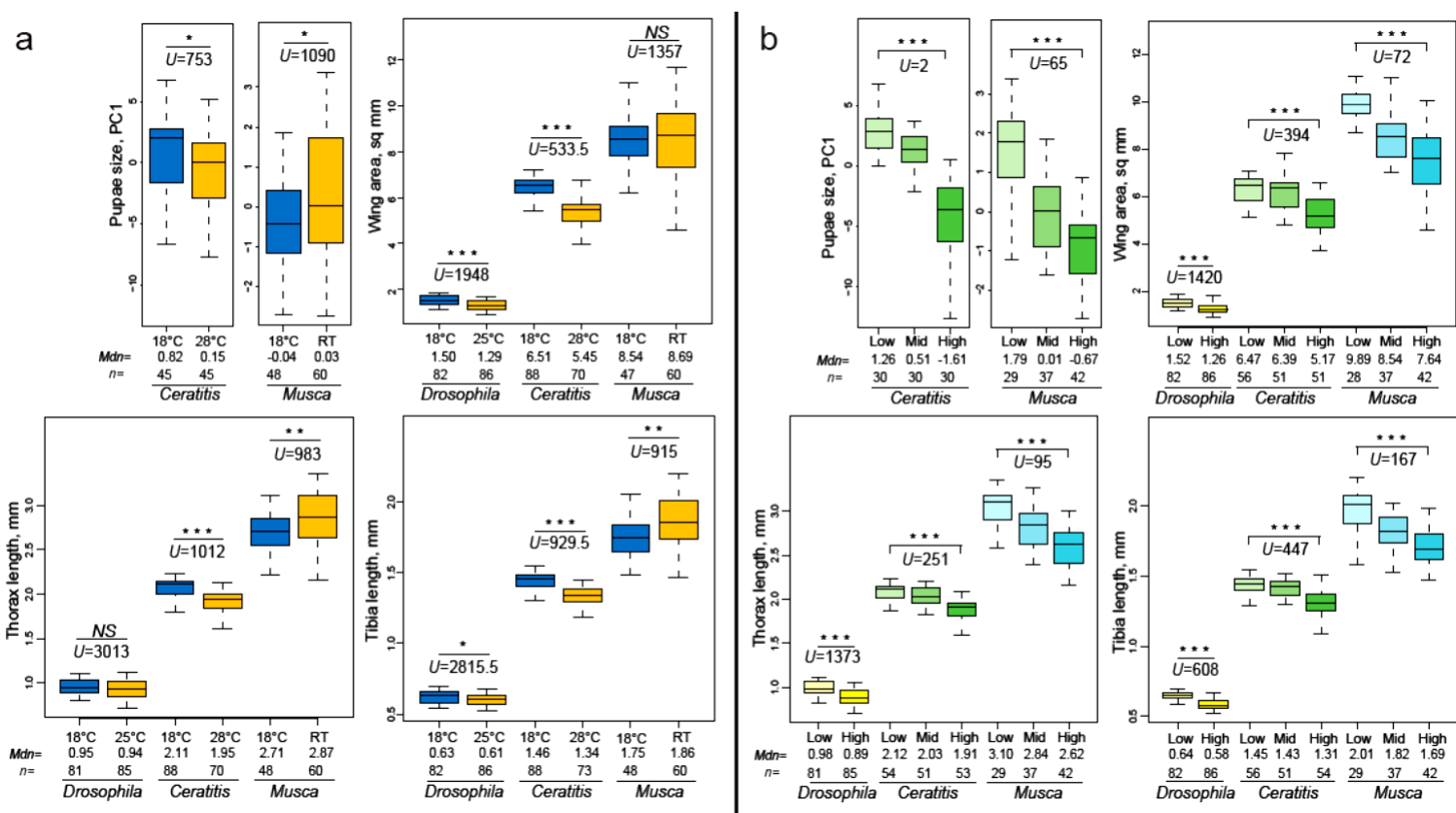

Figure 4.3: Size variation in pupae and different traits in response to changing environmental conditions. Response to different larval densities (a) and rearing temperatures (b). RT means room temperature. Low, Mid and High corresponds to the low, middle and high densities of larvae. Error bars show the max and min values. Statistical significance was checked with Mann-Whitney $U$-test and shown as NS - non-significant at $p=0.05,{ }^{*}=p<0.05 ;{ }^{*}=p<0.005 ;{ }^{* *}=p<0.0001 . M d n$ refers to the median of each group.

\subsubsection{Response to changing environmental conditions is sex-dependent}

Then, I asked whether influence of different rearing conditions on the organ and pupal size was sex dependent. At low temperature $\left(18^{\circ} \mathrm{C}\right.$ for every species), the length and width of wings increased proportionally (with the same rate of change) for both sexes when density decreases (blue and pink numbers in Table 4.2). 
Table 4.2: SSD depends on the environment. Absolute measurements and difference between males and females in the wing length, wing width and thorax length. Comparison of male (blue numbers) and female (violet numbers) traits between groups with varying density. RT refers to room temperature.

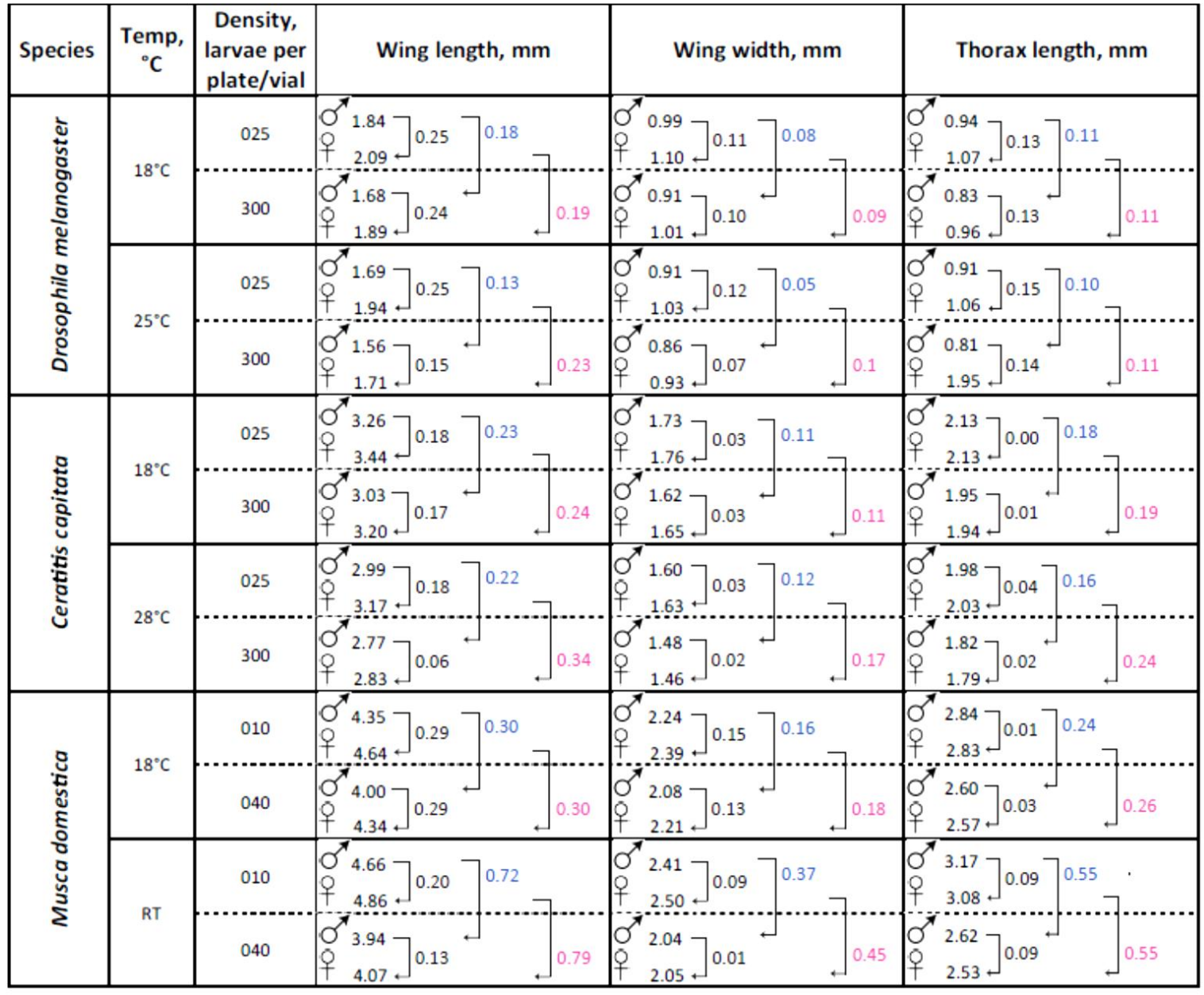

Accordingly, male-female differences in the wing width and length remained constant at both rearing densities (black numbers in Table 4.2 ) at $18^{\circ} \mathrm{C}$. In contrast, at higher temperature, the length and width of female wings changed more in response to the rearing density than those of males (blue and pink numbers in Table 4.2). For instance, the wing width increased twice as much in Drosophila females than in males (i.e. a change of $0.05 \mathrm{~mm}$ in males vs. $0.1 \mathrm{~mm}$ in females) when flies are raised at $25^{\circ} \mathrm{C}$. However, the relative increase differs between species. In Drosophila, wing size increased by $\approx 50 \%$, while in Ceratitis the increase was lower, with $\approx 30 \%$. In line with the increase, the relative male-female differences in the wing width and length was more pronounced in non-crowded conditions (black numbers in Table 4.2) at high temperature. Interestingly, the sex-specific response to environmental conditions was only observed for the linear wing measurements and not for the WA (data not shown), suggesting significant changes in the overall 
wing shape in these species in different conditions (see Chapter 5: Variation of wing shape in different fly species, sections 5.2.4 and 5.2.5).

Besides linear wing measurements, I observed a sex-dependent response in the thorax length in Ceratitis with female thoraces proportionally elongated with decreasing density at $18^{\circ} \mathrm{C}$, and $33 \%$ more than proportional at $28^{\circ} \mathrm{C}$ (blue and pink numbers in Table 4.2). In Musca, the thorax length increase was proportional for males and females at both temperatures $\left(18^{\circ} \mathrm{C}\right.$ and RT), pointing that both temperatures were similarly "cold" for the chosen Italian strain. Also, no effect of the sex on the thorax length in different rearing conditions was observed in Drosophila (Table 4.2), what was supported by the finding that the strain we used in this study seems to be insensitive to the changing rearing temperature in the thorax length. In contrast to the wing and thorax variation, the tibia length did not show sex-dependent changes to rearing conditions. At both temperatures and in all analysed densities, changes in size were proportional for males and females in all species.

\subsubsection{Evolutionary and static allometries for thorax, tibia, and wing size}

Allometries describe scaling relationships between given traits at different evolutionary or developmental stages. They are usually classified into three types, which are ontogenetic, static and evolutionary (Cheverud 1982; Schlichting and Pigliucci 1999). In this study, I focused on the static and evolutionary allometries. The first one describes the relative size of thorax, tibia and wing among individuals of the same species, while the latter compares the relative size of organs among different species at the same stage of development.

I found that the allometric coefficient varied among traits and environmental conditions, but remained similar between species (Figure 4.4). In all conditions, thorax grew slightly hyperallometrically relative to the absolute body size $(b<0.577)$, while the WA showed a strong hyperallometric relationship $(b>0.577)$. Growth of tibia was close to isometric at different rearing densities. Also, at different rearing densities the observed scaling relationships were temperature independent for all three species. In contrast, the allometric coefficient for different temperature regimes was density dependent in Ceratitis and Musca, and to a lesser extent in Drosophila. 


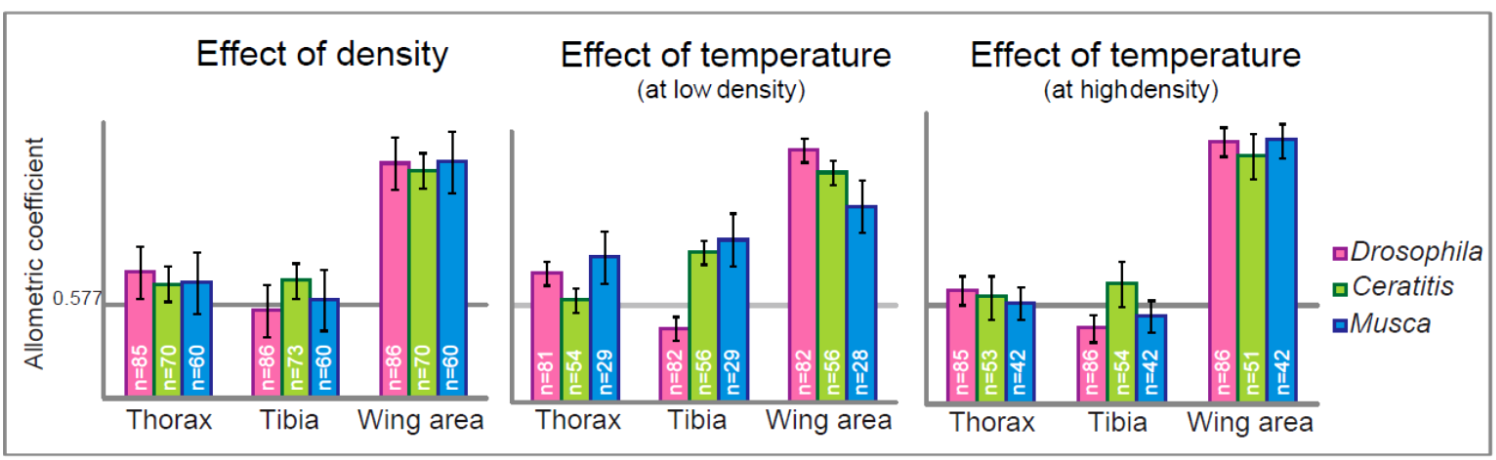

Figure 4.4: Allometric coefficients for different traits. Allometric coefficient $b=0.577$ indicates isometric growth. Traits with $b<0.577$ scale hypoallometricaly with the overall body size, with $b>0.577-$ hyperallometrically. Error bars indicate \pm SD.

\subsubsection{Estimators of the absolute body size in Ceratitis capitata and Musca domestica}

Thorax, tibia and wing sizes are widely used as estimators of the Drosophila body size, because they are known to be highly correlated among each other and with the pupal size, which is supposed to best represent the body size because it is a stage when flies stop feeding and, therefore, do not increase in mass and size (Shingleton et al. 2008).

To define adult body parts that can easily be used to estimate the whole body size of Ceratitis and Musca flies, I computed Spearman's rank correlation coefficients for every pair of the analyzed measurements. For most body parts we found a high correlation (>80\%) in Musca. Only for comparisons between the tibia length and wing measurements, the correlation dropped to 72-75\%. In Ceratitis, correlation coefficients were much lower in general and especially between the measured traits and pupal size (PC1, for details see Materials and Methods 3.3.2.1). Since the correlation of body parts and the pupal size was rather low in Ceratitis, I combined measurements of thorax and tibia with the WA and computed a single BSC out of these three. The correlation of the pupal size with this BSC became 77\%, and correlation coefficients with other body parts increased as well. In case of Musca, computation of the BSC resulted in the highest correlation with the pupal size (97\%) and correlation of the BSC with other parameters remained $\geq 88 \%$ (Table 4.3). 


\begin{tabular}{|c|c|c|c|c|c|c|c|c|}
\hline & \multicolumn{8}{|c|}{ Ceratitis } \\
\hline \multirow{5}{*}{ 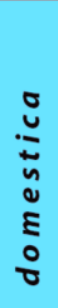 } & & $\begin{array}{l}\text { Pupae size } \\
\text { (PC1) }\end{array}$ & $\begin{array}{l}\text { Thorax } \\
\text { length }\end{array}$ & $\begin{array}{l}\text { Tibia } \\
\text { length }\end{array}$ & $\begin{array}{l}\text { Wing } \\
\text { length }\end{array}$ & $\begin{array}{l}\text { Wing } \\
\text { width }\end{array}$ & $\begin{array}{l}\text { Wing } \\
\text { area }\end{array}$ & BSC \\
\hline & $\begin{array}{l}\text { Pupae size } \\
\text { (PC1) }\end{array}$ & & 0.76 & 0.70 & 0.72 & 0.74 & 0.76 & 0.77 \\
\hline & $\begin{array}{l}\text { Thorax } \\
\text { length }\end{array}$ & 0.94 & & 0.82 & 0.78 & 0.85 & 0.86 & 0.93 \\
\hline & $\begin{array}{l}\text { Tibia } \\
\text { length }\end{array}$ & 0.90 & 0.89 & & 0.71 & 0.82 & 0.88 & 0.95 \\
\hline & $\begin{array}{l}\text { Wing } \\
\text { length }\end{array}$ & 0.87 & 0.81 & 0.72 & & 0.93 & 0.86 & 0.80 \\
\hline \multirow{3}{*}{$\begin{array}{l}\sigma \\
u \\
u \\
\Sigma\end{array}$} & $\begin{array}{l}\text { Wing } \\
\text { width }\end{array}$ & 0.90 & 0.83 & 0.75 & 0.94 & & 0.95 & 0.90 \\
\hline & $\begin{array}{l}\text { Wing } \\
\text { area }\end{array}$ & 0.90 & 0.84 & 0.74 & 0.98 & 0.97 & & 0.95 \\
\hline & BSC & 0.97 & 0.97 & 0.93 & 0.88 & 0.90 & 0.91 & \\
\hline
\end{tabular}

Table 4.3: Correlations of the pupal size, BSCs and different traits in C. capitata and M. domestica. Spearman's rank correlation coefficients were computed for each pair of variables $(p<0.05)$. BSC refers to the body size coefficient.

\subsubsection{Variation of the relative wing size}

In Section 4.2.3, I presented a pronounced plasticity in the wing size in response to varying rearing conditions in D. melanogaster, C. capitata, and $M$. domestica. Since these three fly species are of different adult size (Sections 4.2.2 and 4.2.3), the question is raised whether the relative WA is comparable between these flies. To this end, I corrected the WA for the body size that was defined by the BSC and compared the relative WA values between the species (Figure 4.5). This analysis showed that Ceratitis had the largest relative WA, while wings of Drosophila and Musca were more similar in size. Additionally, I found that variation in the WA increased with the increasing absolute size of the flies.

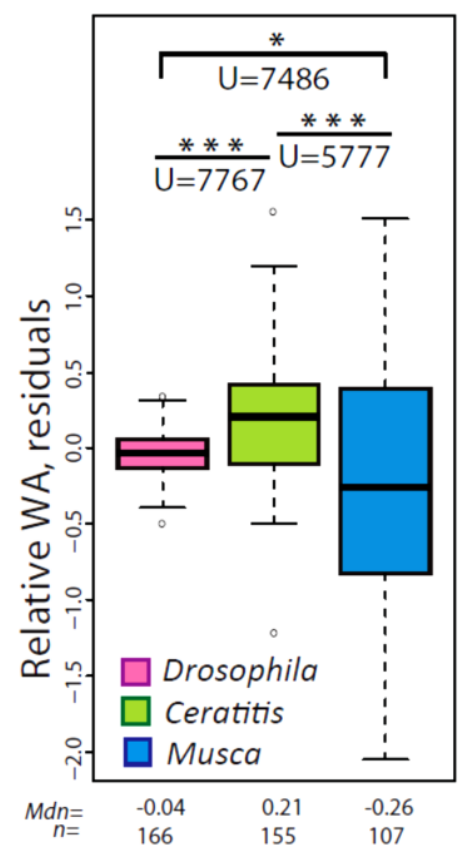

Figure. 4.5: Distribution of the relative size of Drosophila, Ceratitis, and Musca wings. Relative size of wing obtained from WA after correction for body size. Statistical significance was checked pair wise with Mann-Whitney $U$-test and shown as ${ }^{*}=p<0.05 ;{ }^{* *}=p<0.0001$. Error bars indicate the max and min values, circles are outliers. $M d n$ refers to the Median of each group. 


\subsubsection{Ceratitis wings are mainly bigger due to the cell size}

In the previous section, I showed that the relative wing size varied among the analyzed flies. In the next step, I questioned what was the source of this variation: size of cells or the cell number. It was previously shown that wing membranes are covered on both sides with small bristles, which are clearly visible under a microscope. In freshly eclosed flies, it is possible to see that every epidermal cell of the wing membrane produces only one bristle (Dobzhansky 1929). Thus, for a rough estimation of the cell number, I counted bristles along the wing width in Drosophila and Ceratitis wings (see Chapter 3: Materials and Methods, Section 3.3.2.4 for details). An example of such counting is also shown in Figure 4.6 and more details can be found in Appendix 11.2, Table 11.1 in there.
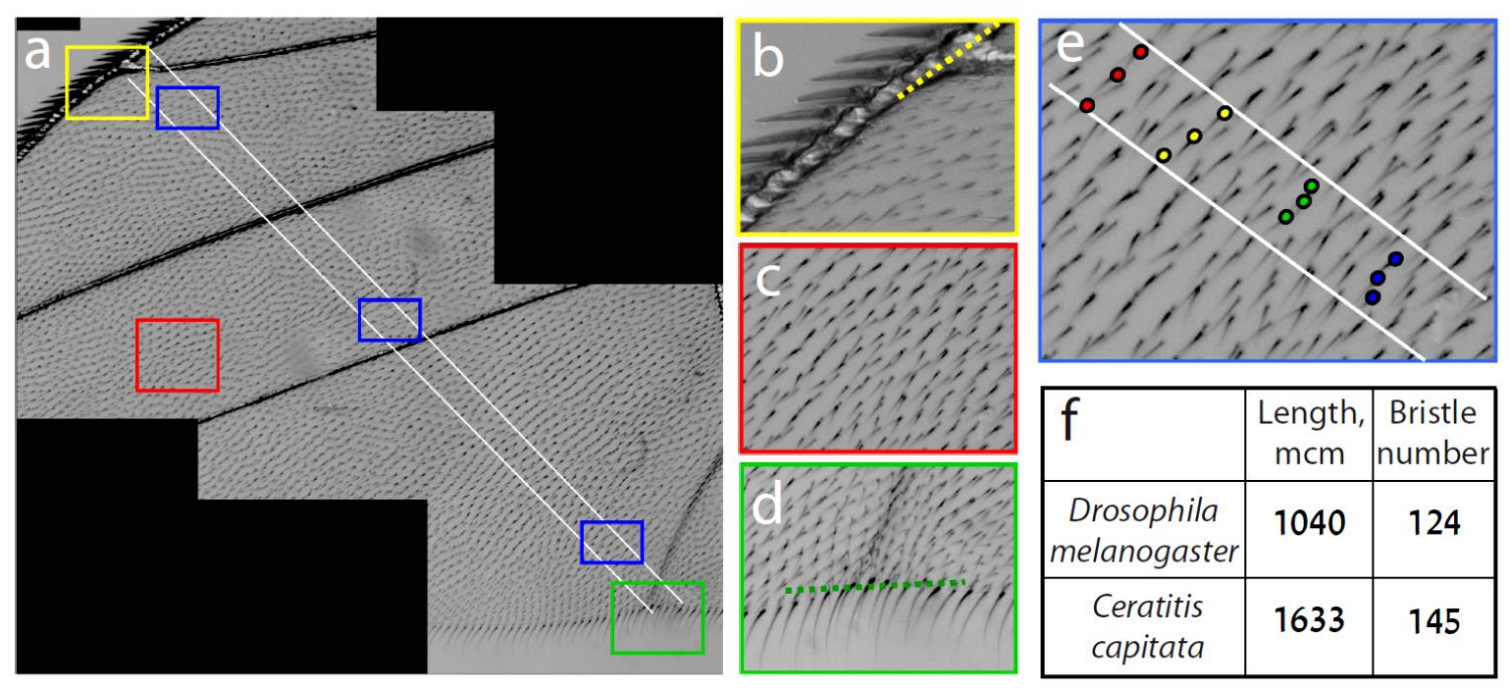

Figure 4.6: Estimation of the bristle number along the wing width line. A fragment of a Drosophila wing is shown as an example to demonstrate the area definition ( $a, b$, and $d$ ), presence of bristles in different areas (b, c and d), estimation of rows of bristles in the defined area (e), and the result (f). Panels (b), (c) and (d) show high magnifications of the areas defined with the respective color in the panel (a). The area where bristles were counted was restricted with two white lines ( $a$ and e), yellow and green dotted lines (b and d) show the upper and the lower borders of the area. Blue boxes show the areas taken for estimation of rows of bristles. The panel (e) shows a high magnification of the middle blue box from the panel (a). Dots in different colors show that on average there are three rows of bristles in this area (e). Length in mcm shows an average length of the ten estimated wings for each species, bristle number is an average number of bristles in one row along the wing width line.

Such counting showed that the difference between the two species is $14.5 \%$ (124 vs 145 bristles) in the cell number, while Ceratitis wings are 36\% larger than those of Drosophila. This led me to the preliminary conclusion that Ceratitis wings were mainly bigger due to larger cell size 
(21.5\%), while the cell number accounts for only a minor variation in size. However, to make the final statement, my primary conclusion should be tested with other more precise methods. And one of such methods could be dissociation of pupal wings in an Elastaze solution, counting the cell number, and estimation of the size and amount of DNA with fluorescence-activated cell sorting.

\subsubsection{Wing loading}

Wing loading is a parameter that describes how much weight can be carried per area of a wing. This is a crucial parameter to estimate performance of the wing. Heavily loaded wings are usually small compared to the weight of an object/animal. In contrast, lightly loaded wings tend to be relatively large. Generally, heavily loaded wings are better suited for the high-speed flight and high landing speed (Tennekes 2009). Thus, in order to estimate forces that are involved in the flying process in our species, I calculated the wing loading coefficient for each fly. I found that the coefficient value was irrespective of the relative wing size of the species, but positively correlated with the absolute body size of the flies (Figure 4.7).
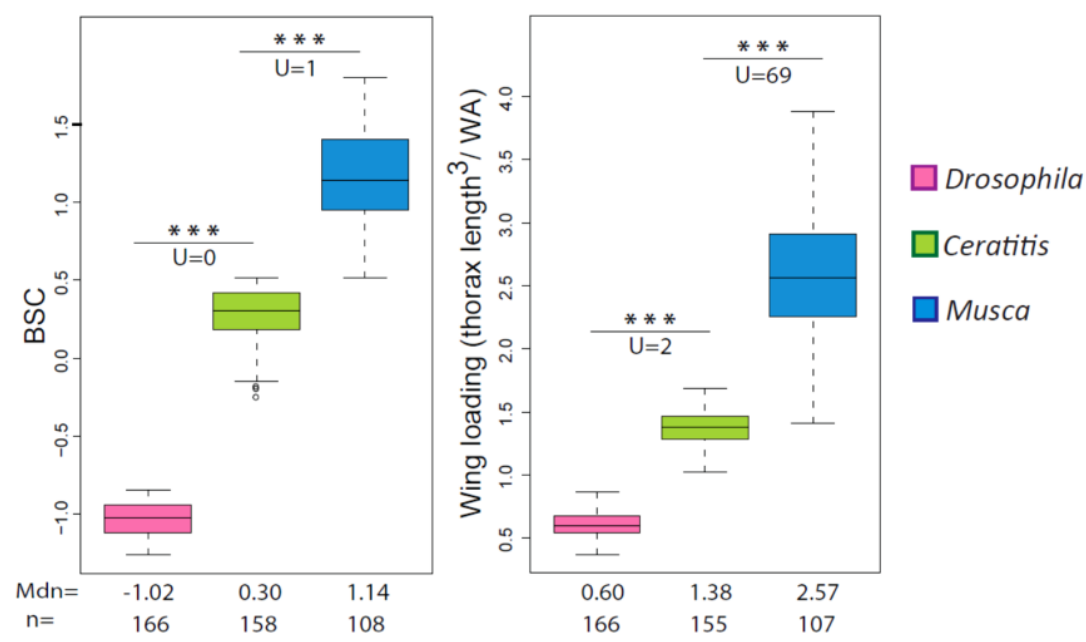

Fig. 4.7: Wing loading increases with the increasing absolute body size. The absolute body size of the flies plotted as the BSC (A) and wing loading (B) in D. melanogaster, C. capitata, and M. domestica. Statistical significance was tested pair wise with Mann-Whitney $U$-test. Error bars indicate the max and min values, $M d n$ refers to the Median of each group, ${ }^{* * *}=p<0.0001$.

I also found a similar trend when the pupae weight or thorax length was considered as estimators of the body size instead of the BSC. The positive correlation with the thorax length retained in different environmental conditions (Figure 4.8). 

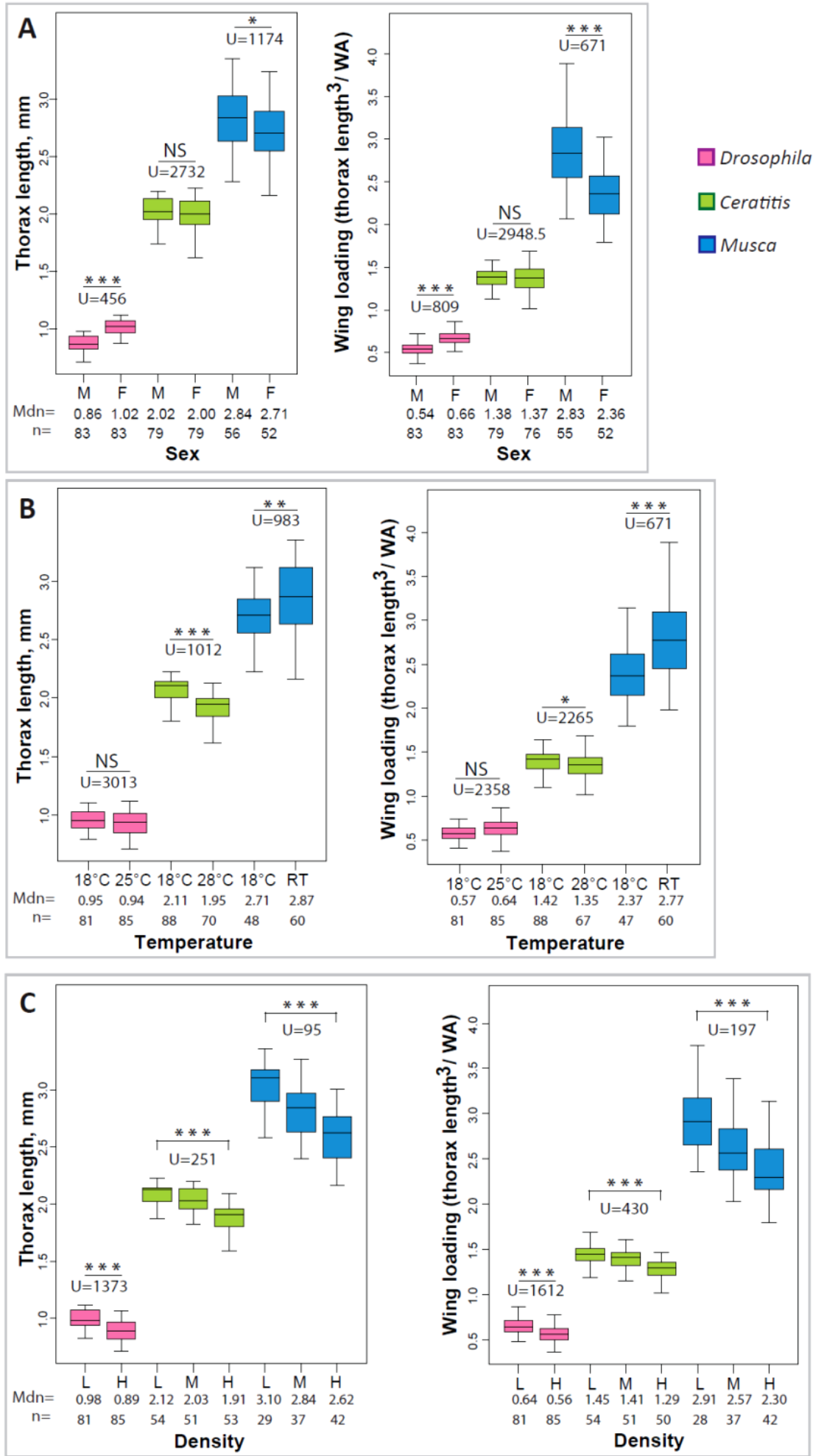

Figure 4.8: Wing loading and the thorax length in males and females in dipteran species grown in different conditions. The thorax length and wing loading in D. melanogaster, C. capitata, and M. domestica males and females (A), at different rearing temperatures (B) and larval densities (C). Statistical significance was tested pair wise with Mann-Whitney $U$-test and shown as $N S$ - non-significant at $p=0.05 ;{ }^{*}=p<0.05$; ${ }^{* *}=p<0.005$ and ${ }^{* * *}=p<0.0001$. Error bars indicate the max and min values. In the panel (A), M refers to males and $\mathrm{F}$ refers to females. In the panel $(\mathrm{C})$, the letters $\mathrm{L}, \mathrm{M}$, and $\mathrm{H}$ refer to low, middle and high density. $M d n$ is the Median of each group. 


\subsection{Discussion and future work}

\subsubsection{Estimation of the wing size}

Centroid size is often used as a measure of the overall size of an organ, e.g. wing, or the whole specimen (Klingenberg et al. 1998; Schachter-Broide et al.2009; Rodríguez-Mendoza 2011; Prudhomme 2012). In this work, I evaluated whether the WCS can be used for this purposes in the three dipteran species D. melanogaster, C. capitata, and M. domestica. I found that the WCS was well correlated with the WA in two of the tree species, in Drosophila and Musca. However, correlation between these two parameters depended on the experimental design and rearing conditions in Ceratitis. In these species, correlation coefficients varied from 0.44 to 0.88 depending on the experimental setup and from 0.71 to 0.99 depending on the sex. These results demonstrate that in some cases the WCS represents the absolute wing size and can be used instead. However, if design of an experiment implies only one set of certain conditions, the WSC may not reflect the absolute size. In this case, correlation of the WA (or other parameters) and the centroid size should be examined beforehand, as it was done for Ceratitis in this study.

\subsubsection{Sexual size dimorphism in Drosophila, Ceratitis, and Musca}

Sexual dimorphism is a phenotypic difference between male and female individuals of the same species. Sexual dimorphism can be observed in a variety of traits such as body or organ size, body structure and shape, pigmentation or behaviour. Although body and organ size is highly variable in animals, a number of common trends have been observed in large groups. For example, most invertebrates have a female-biased SSD with males being smaller than females (Shine 1979; Shine 1994; Head 1995; Teder and Tammaru 2005). It holds true for D. melanogaster as well (Badyaev 2002; Stillwell et al. 2010) and my results confirm this. In this work, I showed that size differences are already evident in late instars, which are not feeding and don't grow anymore, and reflected in the observed sexual dimorphism in the pupal size.

At the intraspecific level, SSD in the whole body size (Figure 4.2, pupae size) and individual body parts (Figure 4.2) was highly variable in all studied fly species. Interestingly, SSD was not uniform even within species, but depended on external cues. It has been shown that in most species that have a female-biased SSD, female size increases more than proportionally in comparison to males, when flies are compared between different environmental conditions (Santos et al. 1994; Teder and Tammaru 2005). My results demonstrate that this phenomenon is 
also present in Ceratitis and Musca, the two species that belong neither to the female-biased nor male-biased system. These flies rather represent a mixed system of SSD. In this system, both sexes have similar size and only certain body parts appear to be larger in males, while others are larger in females.

It has been proposed that different sensitivity of females and males to environmental conditions could explain the disproportional growth (Teder and Tammaru 2005). Here, I demonstrate that certain conditions, e.g. temperature, affect sensitivity of flies to varying density regimes during the larval development. When flies were raised at cold temperature, size changes were proportional at different rearing densities for both sexes. However, warm temperature stimulated the sex dependent sensitivity (Figure 4.9).
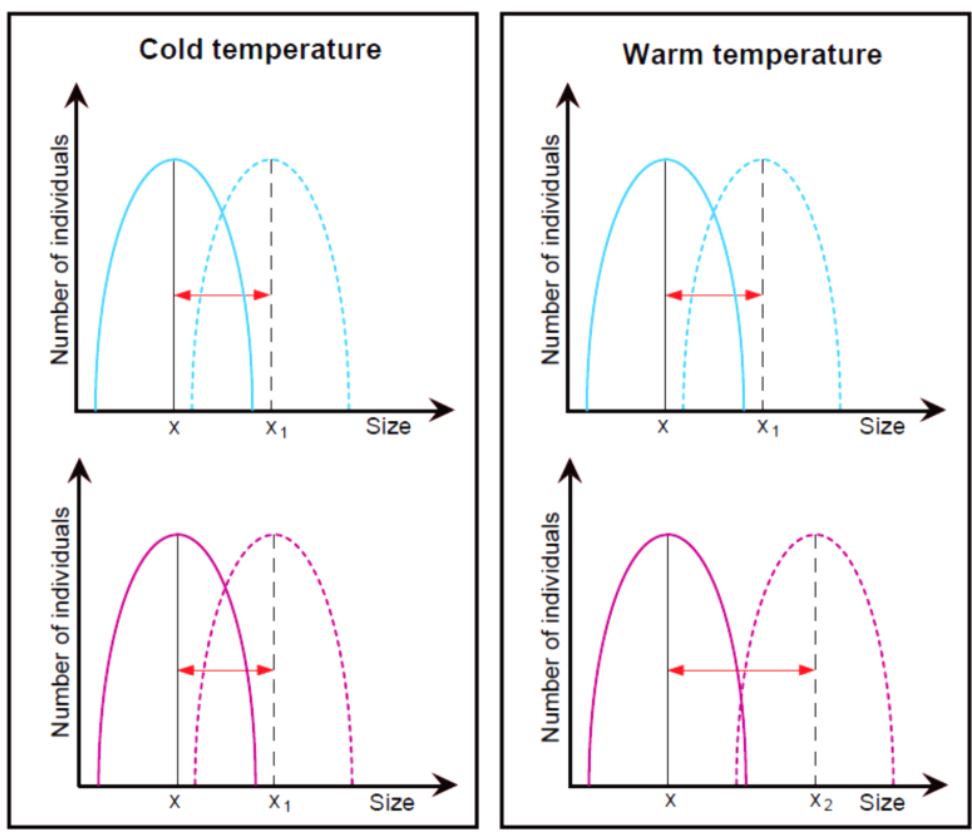

Figure 4.9: Scheme of a size increase in respect with SSD. Scheme of a size increase of male (blue) and female (pink) wings and thoraces at different densities (high - solid line, low - dashed line) for cold and warm temperatures. Red arrows indicate increase of the organ.

This sex dependent sensitivity seemed to be organ or tissue specific, since I observed it for thoraces and wings, but not for tibia measurements. Intriguingly, this observation recapitulates the common origin of the dorsal thorax and wings from the same wing imaginal disc. In contrast, legs develop from a different imaginal disc (Madhavan and Schneiderman 1977; Cowley and Atchley 1990) and this can explain absence of the sex dependant sensitivity in this adult trait. My data suggest that different imaginal discs can react differentially to varying environmental conditions in a sex-dependent manner. 


\subsubsection{Body and organ size: response to different rearing temperatures}

Most poikilotherms follow Bergmann's rule (Ray 1960), and in my work I confirmed a similar trend for Ceratitis (Navarro-Campos et al. 2011) and Drosophila (French et al. 1998; Robinson and Partridge 2001). In both species, body size and all measured body parts decreased in size with the increasing rearing temperature. While this trend was highly significant for all traits in Ceratitis, in Drosophila changes in the thorax and tibia lengths were present but not significant. At the same time, wings remained temperature sensitive and followed Bergmann's rule. Although thoraces and wings develop from the same larval tissue, it has been shown that timing of temperature sensitivity differs between them. The rearing temperature has a cumulative effect on the thorax size throughout the larval life, while the wing size is only affected during the third larval instar and most of the pupal stage (Pantalouris 1957; French et al. 1998). Intriguingly, the Drosophila strain used in my survey $\left(\mathrm{w}^{1118}\right)$ was kept at $18^{\circ} \mathrm{C}$ for more than 20 years. These cold adapted flies might have accumulated a temperature insensitivity of the leg imaginal discs and the part of the wing imaginal disc that contributes to the dorsal thorax. Such a striking alteration was also known for the body size regulation in the nematode Caenorhabditis elegans, where a single nucleotide substitution resulted in worms that were insensitive to the rearing temperature (Kammenga et al. 2007).

Since the compliance of Bergmann's rule seems to be retained by selection rather than physiological or thermodynamic constraints (Scheiner and Lyman 1991), the phenotypic plasticity of the body size in response to temperature is heritable and can be artificially selected (Scheiner and Lyman 1989; deMoed et al. 1997). This means that rearing flies at constant laboratory conditions for a long time (i.e. 20 years at $18^{\circ} \mathrm{C}$ ) might allow accumulation of mutations that resulted in the elimination of the temperature sensitivity in this strain. Thus, I believe that D. melanogaster $\mathrm{w}^{1118}$ warrant further investigations, which can add to our understanding of how wings attain their final size and how wing gene networks differ from those of thorax.

Although there are known exceptions from Bergmann's rule (Atkinson 1994; Kingsolver and Huey 2008), my finding that Musca showed a positive thermal reaction norm for the size of all measured body parts was unexpected because natural populations of the species follow Bergmann's rule with a negative thermal reaction norm (Bryan 1977; Alves and Bélo 2002). In contrast to previous publications, I used a laboratory strain that was recently collected in Italy (see Chapter 3: Materials and Methods, Section 3.1 for details). While room temperature $\left(22 \pm 2^{\circ} \mathrm{C}\right)$ was 
well-suited to keep stocks of this strain, the rearing temperature of $18^{\circ} \mathrm{C}$ could be too stressful for these flies, especially at low densities. Thus, I believe that the observed opposite reaction might be induced by cold stress, and in this condition with low survivorship, the temperature-size rule cannot be properly applied (David and Clavel 1967; Kingsolver and Huey 2008). Another possible explanation can be a shift in the diet. The strain used for this study was only recently established for the constant rearing under laboratory conditions. During this time, flies were provided with sugar water as food, and this change from the natural to laboratory nutrition might affect the temperature-size rule (Diamond and Kingsolver 2010).

\subsubsection{Body and organ size: response to changing rearing densities}

I found a clear influence of the rearing density on the final body size and size of individual body parts (Figure 4.3b). For Drosophila, I analysed only two conditions that are known to be density extremes for this species. In this work, I confirmed the previously observed trend that flies are smaller when they are raised at higher densities (Santos et al. 1994). I found the same trend for Ceratitis and Musca. While the body and organ size in Ceratitis remained rather stable between the low- and mid-density conditions, the size of all measured traits decreased clearly when the middle- and high-density conditions were compared, suggesting a non-linear relationship between the density and size. However, in Musca, I observed a linear relationship for the thorax and tibia lengths, while pupal size and the WA already showed the most obvious response between the low and middle density conditions (Figure 4.3b). There are known several previous attempts to establish if reduction in size is linearly dependent on density or not. The first results suggested that body size, measured as dry weight of adult flies, was non-linearly reduced in increasing density (Miller and Thomas 1958). Later, Santos et al. (1994) found that body size, measured as the thorax length, had a linear response to the changing density. My data show that the density response is highly variable and that different body parts have a potential to change with a different rate. Thus both linear and non-linear relationships are likely to occur in different organs and different fly lineages.

Until now, there is no clear model that fully explains the mechanism of the body size regulation under conditions of different crowding. It has been proposed that this phenomenon could be explained by a possible pheromone regulation (Shingleton et al. 2009) and regulation via oxygen level in the media with hypoxia occurring at high densities (Biddulph and Harrison 2014). 
Increased concentration of waste products as a consequence of crowding can also be recognized and interpreted by larvae during growth. The data obtained in this study represents an excellent starting point to further explore genetic and physiological mechanisms underlying the organspecific size regulation in response to changing rearing densities in a comparative way.

\subsubsection{Body size estimators and growth scaling}

Body size has been shown to correlate with fundamental life history traits, mating behaviour and mating success (Burk and Webb 1983). Therefore, it is of general interest to estimate body size of individuals originating from different wild populations to infer various environmental and ecological conditions. This is even more important for insects that represent serious pests, such as the three dipteran species studied here.

The final adult body size of a fly is a consequence of growth during the feeding larval stages, while the 'wandering larva' and immobile pupa stage do not increase in mass and volume anymore. Therefore, these two stages are good estimators of body size (Churchill-Stanland et al. 1986; Shingleton et al. 2008; Stillwell 2011). However, in wild populations, the access to different stages of the lifecycle is highly limited. As an alternative, different parts of the adult body are often used as size estimators in different studies. For instance, it has been extensively shown that adult thorax, tibia and wing size are highly correlated with the absolute body size in Drosophila (Cavicchi et al. 1989; Pitnick and Markow 1995; de Moed et al. 1997; Kacmarczyk and Craddock 2000). In fact, these correlations were even extrapolated to other dipterans in some cases (Gleiser et al. 2000; Navarro-Campos et al. 2011). Here, I show that body part correlations in other dipteran flies are not always consistent with correlations known from Drosophila. For instance, the tibia length, which is widely used in Drosophila studies as a body size estimator, exhibits a low correlation with other body size measurements in Ceratitis. Similarly, the tibia length alone is of limited value to estimate the body size of Musca flies.

Additionally, I found different scaling relationships between the size of individual traits and overall body size. A study on scaling relationships in Drosophila melanogaster Oregon R indicated that changes in traits were not always proportional with the absolute body size and the relative size of each trait could change dramatically in different rearing conditions (Shingleton et al. 2009). I confirmed these results in our Drosophila strain and provided additional evidence supporting the previous suggestion by Shingleton et al. that different genotypes respond differently to the 
same environmental variables. However, although I saw differences in allometries among different species, I still found consistent trends. For instance, the effect of variable temperature seemed to be masked at high densities, suggesting that rearing density was more crucial than temperature. This assertion was further supported by steady and uniform size changes under conditions of variable density, while size changes resulting from different rearing temperatures were more unstable and less universal (Figure 4.4).

Altogether, my data suggests that the use of a single body part to estimate the entire body size might result in wrong assumptions. Therefore, I propose to use a BSC computed from measurements of several traits. As an alternative, pupal traits (volume or dry weight) should be used since they represent the body size best and remain unaffected by the environment.

\subsubsection{Variation of wing size and wing loading}

In this part of my work, I showed that the relative wing size was not uniform among species of the same order. More specifically, when I corrected wing size for body size, I found that Ceratitis wings were relatively larger than those of Drosophila and Musca. Preliminary, I conclude that the observed variation occurred due to both the cell size and cell number. I found that the cell size has a higher impact on the variation $(\sim 25 \%)$ than the cell number $(\sim 15 \%)$. However, this preliminary conclusion is rather superficial because it is based on rough estimations. Therefore, it would be interesting to test the hypothesis in details with more powerful methods.

Since I found differences in the relative wing size, I estimated whether they might influence aerodynamics of the three species. It is known that wing loading, i.e. the ratio of the WA to body size (for details see Materials and Methods 3.3.2.3), can serve as an estimator of takeoff and landing speeds as well as flight speed. Because a smaller WA causes less drag, high-speed flight is only possible with heavily loaded wings. This type of wings also requires large take-off and landing speeds because the relatively small wing cannot generate lift sufficient for keeping the animal aloft at low speed. In contrast, bigger and lightly loaded wings are better suited for situations demanding low take off and landing speeds. This, however, reduces the speed of the animal. Additionally, wing loading can affect maneuverability during flying. Thus, animals with high wing loading cannot perform quick maneuvers and flips. (Tennekes 2009) Interestingly, the observed significant difference in wing size did not affect wing loading in the three studied species. Even though Ceratitis had the largest relative wing size, wing loading was intermediate in 
this species. Similar to Starmer and Wolf (1989), I found that the wing loading value increases with body size and it was positively correlated with the thorax length. Hence, Drosophila had the smallest wing loading, while Musca had the largest. In accordance with this finding, it has previously been shown that D. melanogaster performs rapid turns and saccades (Fry et al. 2003), while wing loading coefficients calculated for Ceratitis and Musca suggest that these two species might be less dynamic and rapid maneuvers are not frequent events in their flight. Additionally, it has been shown that reduced wing loading might have an impact on the wing beat frequency (Pétavy et al. 1997), suggesting that differences in this trait could be observed between the three dipteran species studied here.

Altogether, I found that three fly species belonging to the same insect order exhibit significant difference in the overall body size and show a clear variation in the wing size. Consequently, these closely related species represent an excellent system to further study the impact of this size difference on potentially adaptive trait such as flight performance. 


\section{VARIATION OF WING SHAPE IN DIFFERENT FLY SPECIES}

There is an incredible variety of forms and shapes that can be seen in nature. As one of examples of such variation, one can mention a sharp distinction of shapes of incest wings. This peculiar feature has been widely used by researchers in morphology and taxonomy of organisms (Comstock 1893; Plowright and Stephen 1973; Rohlf 1993). For a long time, shape was considered as a descriptive parameter only. In the last century, however, there was a rapid development of methods to quantify the morphological variation (Huxley 1924; Huxley 1932; Gould 1966). In the last two decades of that century, the rise of geometric morphometrics provided a basis for the current methods for analysing variation in oganismal shape in both animals and plants (Gumiel et al. 2003; Klingenberg 2003; Viscosi and Cardini 2011; Mitteroecker et al. 2013; Sadeghi and Dumont 2014; Guan et al. 2015; Torres and Miranda-Esquivel 2016). In this study, I applied geometric morphometrics to describe inter- and intraspecific shape differences of wings and reveal shape changes as a consequence of different rearing conditions: temperature and density. Results of this analysis are described in this chapter and reviewed in the following discussion. Later, they will be submitted as a manuscript to PLOS One in co-authorship with Dr. Ernst A Wimmer and Dr. Nico Posnien.

\subsection{Geometric morphometrics of fly wings}

Insects represent a large group of animals that have the ability of a powered flight. This led to a high variation and great ecological success of insects allowing them to occupy new ecological niches including air. Flying helps them to surmount long distances in a relatively short time, facilitating to basic tasks such as finding mating partners and food resources. However, in many insect species, wings are not exclusively used for flying. They acquired special significance in other essential processes such as mating or defense. For instance, a number species developed different kinds of courtship songs, which help females to choose a mating partner judging the size and vigor of the male (Burk and Webb 1983; Partridge et al. 1987). Some insects learned to mate in the air while flying (Wilkinson and Johns 2005), while others can strike in flight but always land prior to copulation (Murvosh et al. 1964). These different behavioral habits together with the intersexual food competition and reproductive role division cause a constant selective pressure and result in different kinds of sexual dimorphism, including variation in size and shape of insect wings. By example of D. melanogaster, it was shown that size and shape of wings are often 
regulated by the same processes in the wing imaginal discs during patterning and differentiation along the larval and pupal development (Day and Lawrence 2000; Matamoro-Vidal et al. 2015; Testa and Dworkin 2016). This is why in many cases shape comes up as a consequence of size changes and, therefore, these two parameters were considered together for a long period of time (Cowley et al. 1986). Results of the shape analysis were inseparably linked to the wing size variation in a great number of studies. At present, however, development of modern mathematical tools made it possible to distinguish them and analyze size and shape independently (Bookstein 1991; Klingenberg 1996; Bitner-Mathé and Klaczko 1999; Debat et al. 2003; Mitteroecker and Gunz 2009).

\subsection{Sexual shape dimorphism of Drosophila, Ceratitis, and Musca wings}

It was shown that numerous body parts, including wings, can develop to different sizes, and this development is regulated by both internal and external cues (Beadle et al. 1938; Santos et al.1994; Robinson and Partridge 2001; Peck and Maddrell 2005; DiAngelo et al. 2009; Shingleton et al. 2009; Siomava et al. 2016). One of the results of such differential growth is appearance of male- or female-biased SSD, when either male or female sex is relatively larger (Esperk et al. 2007; Fairbairn et al. 2007; Allen et al. 2011). It was also shown that size changes occurring in response to different rearing conditions are sex specific (Teder and Tammaru 2005; Siomava et al. 2016). In female-biased systems, females overgrow males under certain environmental conditions. It results in the disproportional growth of certain body parts, e.g. wings, or the whole animal grow to a larger size (Bonduriansky 2007; Lavine et al. 2015). However, it is not known whether shape of male and female wings differently responds to environmental cues and what the difference would be. This question was not well studied yet and remained open so far. Researchers possess some information regarding the natural variation in different Drosophila species as well as the influence of rearing conditions on wing shape of Drosophila and other insects (Cavicchi et al. 1991; Pezzoli et al. 1997; Moraes et al. 2004; Yeaman et al. 2010). There is also available a number of studies on SShD of wings in various species (Gidaszewski et al. 2009). However, sexual specificity of the response has not been tested and the direction of changes remained unclear. Therefore, I focused on sexual shape dimorphism (SShD) of wings and its variation in response to different environmental cues in the three dipteran species of our study: D. melanogaster, C. capitata, and M. domestica. 


\subsubsection{Wing shape variation in dipteran species}

To estimate differences in wing shape between Drosophila, Ceratitis and Musca, I digitized 11 landmarks on anatomically homologous points in all three species (Figure 3.1). Ten of eleven landmarks marked vein intersections and one landmark was placed on the alula opening. Because size is usually the main factor contributing to shape variation (Klingenberg 2016), I corrected wings for the evolutionary allometry and averaged shape of the left and right wings for every individual. Subsequently, I performed a PCA to visualize the non-allometric component of shape in a scatter plot (Figure 5.1).
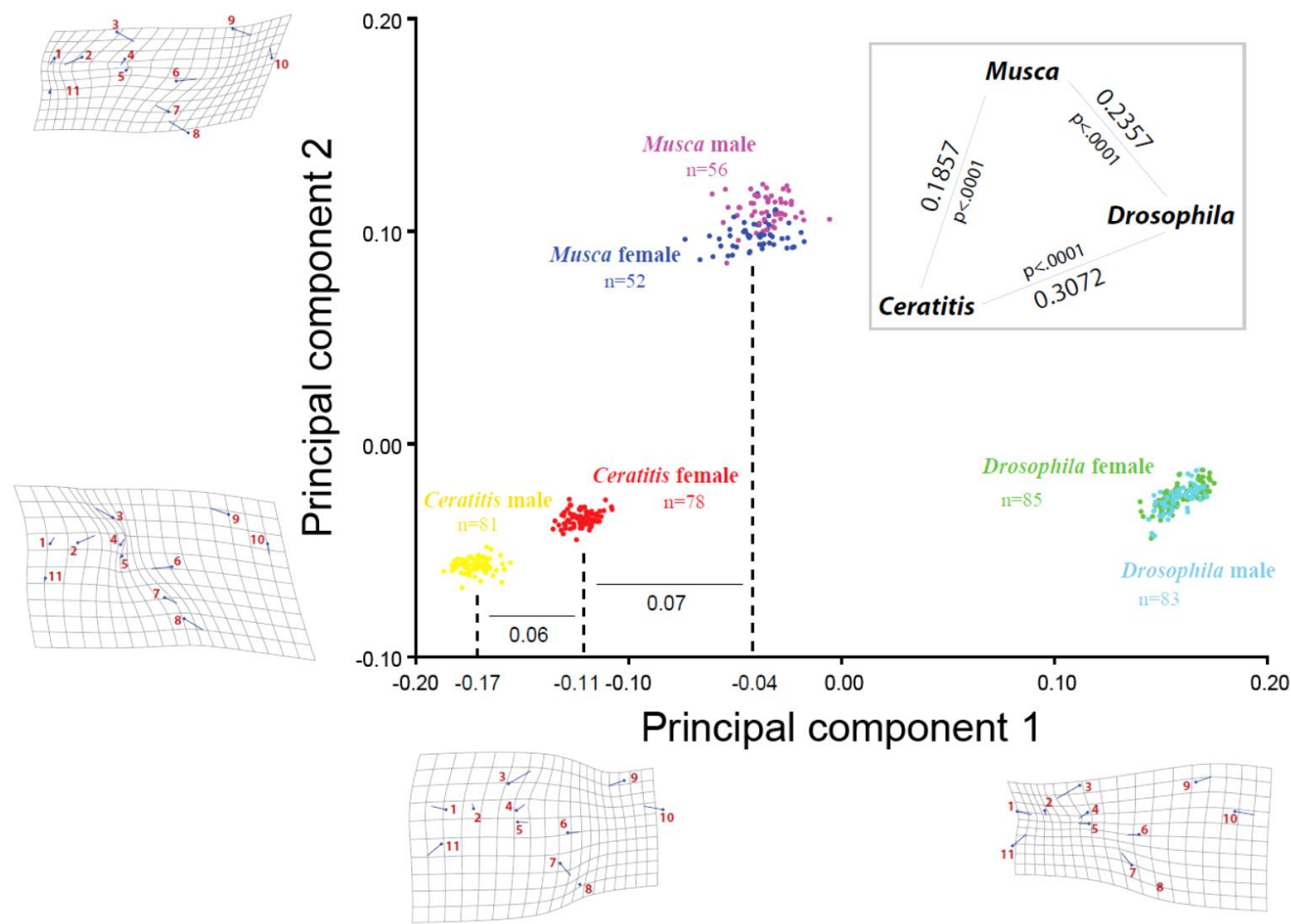

Figure 5.1: Wing shape variation between $D$. melanogaster, C. capitata, and $M$. domestica after size correction. PCA of the shape scatter plot (PC1 and PC2) and associated shape change of the nonallometric shape component of side averaged wings. The TPS deformation grids illustrate shape changes indicating the relative shifts of landmarks 1 to 11 along the axes with the PC scale factor $+/-0.2$. Dots on grids with the respective landmark number (red) indicate the starting point and blue lines connect them to the final shape. Mean values of the shape variance along PC1 and their projections (dashed lines) are shown for Ceratitis males, females, and Musca flies. The inner panel shows morphometric distances between wing shapes in the three species and $p$-values for them. 
I found that the first two PCs accounted for almost $98 \%$ of the variation among landmarks. The main shape difference was reflected in PC1 ( $>80 \%$ of variation) that mainly represented the ratio between the proximal and distal parts of the wing. TPS deformation grids showed that Ceratitis wings were broader in the proximal part (landmarks 1-5, 11), than in the distal (landmarks 6-10). Drosophila wings represented the opposite case with the proximal part being heavily compressed along the anteroposterior axis. Musca had the intermediate morphology being, however, more similar to Ceratitis. Interestingly, along the PC1, Ceratitis male wings $\left(\mathrm{PC}_{\text {mean }}=-0.17\right)$ and Musca wings $\left(\mathrm{PC} 1_{\text {mean }}=-0.04\right)$ were almost equidistant from female Ceratitis wings $\left(\mathrm{PC} 1_{\text {mean }}=-0.11\right)$,

implying a strong sexual dimorphism in Ceratitis that was comparable to the interspecies difference along this axis.

The second significant PC explained $16 \%$ of the variation mainly accounting for the ratio between the length and width of the whole wing. In Ceratitis and Drosophila, landmarks 3, 7, and 8 were shifted from the centre towards the margin, increasing the width of the wing (Figure 5.1, TPS deformation grids along PC2). At the same time, landmarks 2 and 6 were displaced towards the centre, resulting into a shorter wing. Landmarks 9 and 10 additionally increased these effects and made Ceratitis and Drosophila wings more compact in comparison with elongated Musca wings. Along this axis, sexual dimorphism in Ceratitis was less prominent than the interspecies difference with Musca. This shape variation resulted into the largest morphometric distance between Ceratitis and Drosophila (Procrustes distance $=0.3072 ; p<0.0001$ ), with Musca being closer to Ceratitis (Procrustes distance $=0.1857 ; p<0.0001$ ) than to Drosophila (Procrustes distance $=0.2357 ; p<0.0001)($ Figure 5.1, the inner panel).

\subsubsection{Growth trajectories and static allometry}

One of the widespread methods to study allometry in morphometrics is a multivariate regression of shape on size. This regression tests whether there is statistical allometric variation of shape as a result of changes in size (Monteiro 1999). One of the limitations of this test that can be imposed by data is a range of sizes used for the analysis. If the range of sizes in not broad enough, this will lead to a computation of only a short section of the allometric trajectory and result into underestimation of proportions of shape that allometry accounts for. It is strongly suggested to include the smallest and the largest specimens from experiments, which represent the extremes of the size distribution, because they may contribute substantially (Klingenberg 2016). To avoid this 
limitation of the method, I generated differently sized flies (see Chapter 3: Materials and Methods, Section 3.2) and included different sizes in the analysis. The sample size, range of wing sizes and its median are shown in the Table 5.1.

Table 5.1: Summary of the fly species and sample size (N) used in the experiment. The WCS is shown for landmarks 1 to 11 for each species, IQR refers to the interquartile range.

\begin{tabular}{|c|c|c|c|c|c|}
\hline Species & Strain & Sex & $\mathbf{N}$ & $\begin{array}{c}\text { Size range } \\
\left(\mathrm{WCS}^{1-11}, \mathrm{~mm}\right)\end{array}$ & $\begin{array}{l}\text { Median }(\mathrm{IQR}) \\
\left(\mathrm{WCS}^{1-11}, \mathrm{~mm}\right)\end{array}$ \\
\hline Musca domestica & ITA1 & $\begin{array}{l}\text { male } \\
\text { female }\end{array}$ & $\begin{array}{l}56 \\
52\end{array}$ & $\begin{array}{l}3.79-5.45 \\
4.18-5.82\end{array}$ & $\begin{array}{l}4.86(4.53-5.10) \\
5.06(4.75-5.34)\end{array}$ \\
\hline Ceratitis capitata & Egypt II & $\begin{array}{l}\text { male } \\
\text { female }\end{array}$ & $\begin{array}{l}81 \\
78\end{array}$ & $\begin{array}{l}2.93-3.93 \\
2.97-4.10\end{array}$ & $\begin{array}{l}3.61(3.46-3.82) \\
3.70(3.56-3.93)\end{array}$ \\
\hline Drosophila melanogaster & $\mathrm{w}^{1118}$ & $\begin{array}{l}\text { male } \\
\text { female }\end{array}$ & $\begin{array}{l}83 \\
85\end{array}$ & $\begin{array}{l}1.64-2.12 \\
1.68-2.39\end{array}$ & $\begin{array}{l}1.89(1.81-2.00) \\
2.18(2.01-2.25)\end{array}$ \\
\hline
\end{tabular}

The multivariate regression was performed for each of the studied species separately for males and females. Plots of size versus shape scores did not perfectly fit to the straight line in some cases (e.g. Musca females) showing that there were other factors than size corresponding to the allometric vector and contributing to the shape variation (Figure 5.2).
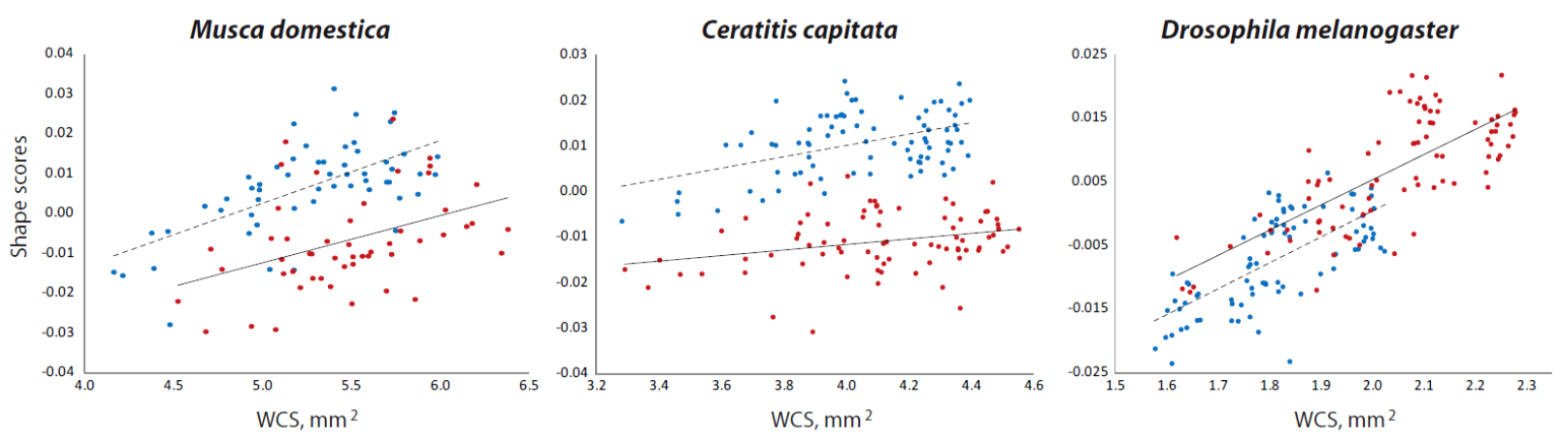

Figure 5.2: Growth trajectories for Drosophila, Ceratitis, and Musca. The growth trajectories are shown with shape scores as a function of the WCS for males (blue dots and dashed lines) and females (red dots and solid lines) in the three species. 
The static allometry was present in both sexes in Drosophila and Ceratitis (Table 5.2). In Musca, however, I did not observe static allometry in females $(p=0.061)$, while in males it was present with high statistical significance $(p=0.0008)$. I also found a statistical difference in growth trajectories between males and females within species at the $5 \%$ level (Table 5.3). The amount of the shape variation occurred due to the variation in size was similar and relatively small in all species (Ceratitis: 4.03\%; Musca: 4.71\%; Drosophila: 6.04\%) when the regression was pooled among sub-groups of temperature and density (Table 5.2). Sex specific regression increased the percentage of the shape variation that occurred due to the size change. 
Table 5.2: Results of the multivariate regression of shape on size for males and females. The shape variation predicted by each regression is shown as a percentage of the total shape variation. $\dagger-$ non-significant at $5 \%$ level.

\begin{tabular}{|c|c|c|c|c|}
\hline Species & Sex groups & $\mathbf{N}$ & \% Predicted & $p$-value \\
\hline \multirow{3}{*}{ Musca domestica } & All & 107 & 4.71 & $<0.0001$ \\
\hline & Males & 55 & 7.70 & 0.0008 \\
\hline & Females & 52 & 3.86 & $0.061^{\dagger}$ \\
\hline \multirow{3}{*}{ Ceratitis capitata } & All & 159 & 4.03 & $<0.0001$ \\
\hline & Males & 81 & 7.00 & 0.0003 \\
\hline & Females & 78 & 5.04 & 0.0025 \\
\hline \multirow{3}{*}{ Drosophila melanogaster } & All & 168 & 6.04 & $<0.0001$ \\
\hline & Males & 83 & 7.04 & $<0.0001$ \\
\hline & Females & 85 & 7.18 & $<0.0001$ \\
\hline
\end{tabular}

Table 5.2: The effect of WCS, sex, and their interaction on wing shape scores, tested with ANCOVA.

\begin{tabular}{|c|c|c|c|c|c|}
\hline Species & Effect & Mean Squares & $\mathbf{F}$ & $p_{\text {slope }}$ & $p_{\text {intercept }}$ \\
\hline \multirow{3}{*}{ Musca domestica } & WCS & 0.001941 & 18.934 & $<0.0001$ & \multirow{3}{*}{$<0.0001$} \\
\hline & sex & 0.006725 & 65.598 & $<0.0001$ & \\
\hline & WCS:sex & 0.000079 & 0.771 & 0.382 & \\
\hline \multirow{3}{*}{ Ceratitis capitata } & WCS & 0.00025 & 6.379 & 0.01260 & \multirow{3}{*}{$<0.0001$} \\
\hline & $\operatorname{sex}$ & 0.01928 & 497.782 & $<0.0001$ & \\
\hline & WCS:sex & 0.00012 & 3.213 & 0.07500 & \\
\hline \multirow{3}{*}{ Drosophila melanogaster } & WCS & 0.013784 & 423.268 & $<0.0001$ & \multirow{3}{*}{$<0.0001$} \\
\hline & sex & 0.00066 & 20.257 & $<0.0001$ & \\
\hline & WCS:sex & 0.000001 & 0.038 & 0.846 & \\
\hline
\end{tabular}


Total SShD
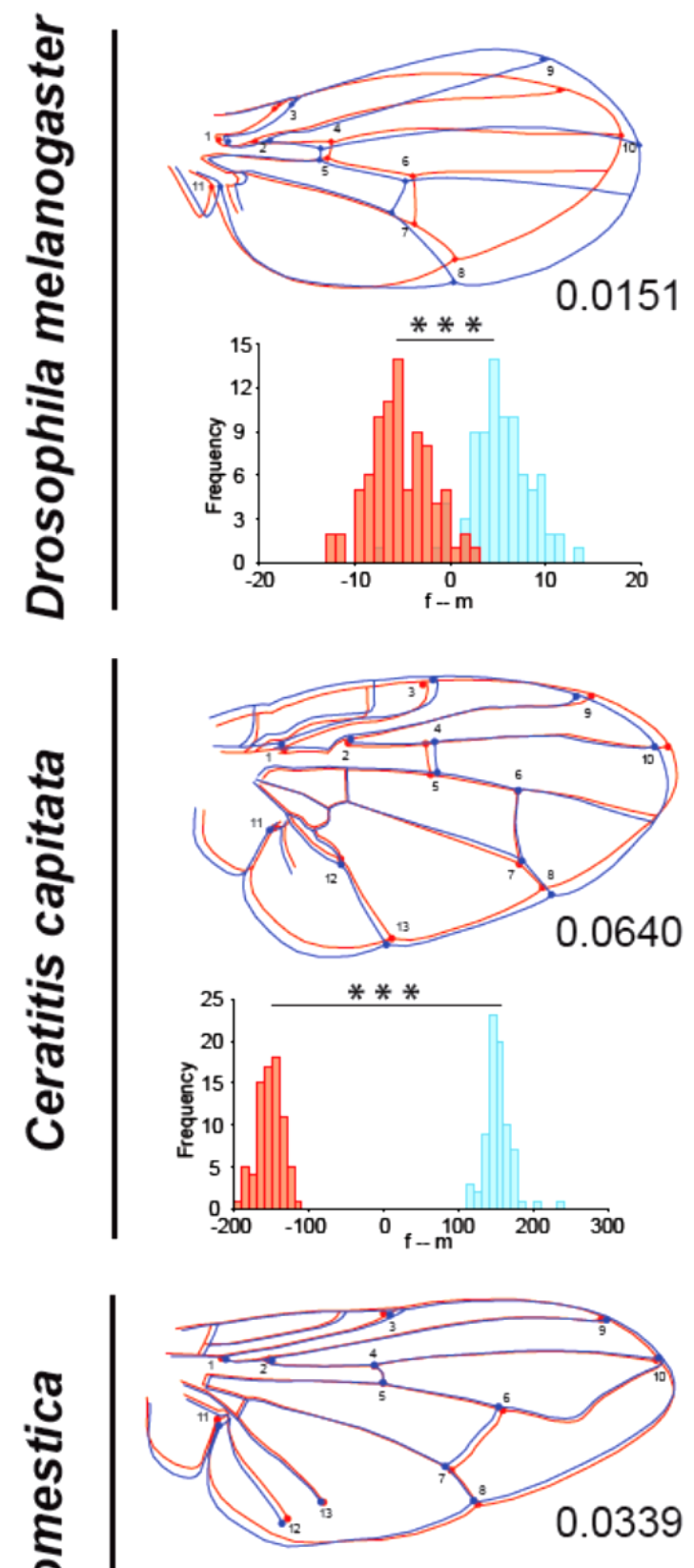

Figure 5.3: Sexual shape dimorphism in wing shape. The total and non-allometric SShD in D. melanogaster (scale factor 9, $n=168$ ), C. capitata (scale factor 1, $n=154$ ) and M. domestica (scale factors 1 , $n=107$ ). Wing outlines represent differences between male (blue) and female (red) average wing shapes. The magnitude of SShD is indicated in units of Procrustes distance with the corresponding p-values $\left({ }^{\star} p<0.05\right.$; $\left.{ }^{* * *} p<0.0001\right)$. Histograms with the distribution of discriminant scores show the shape separation into two distinct groups for each species.

\section{Non-allometric SShD}
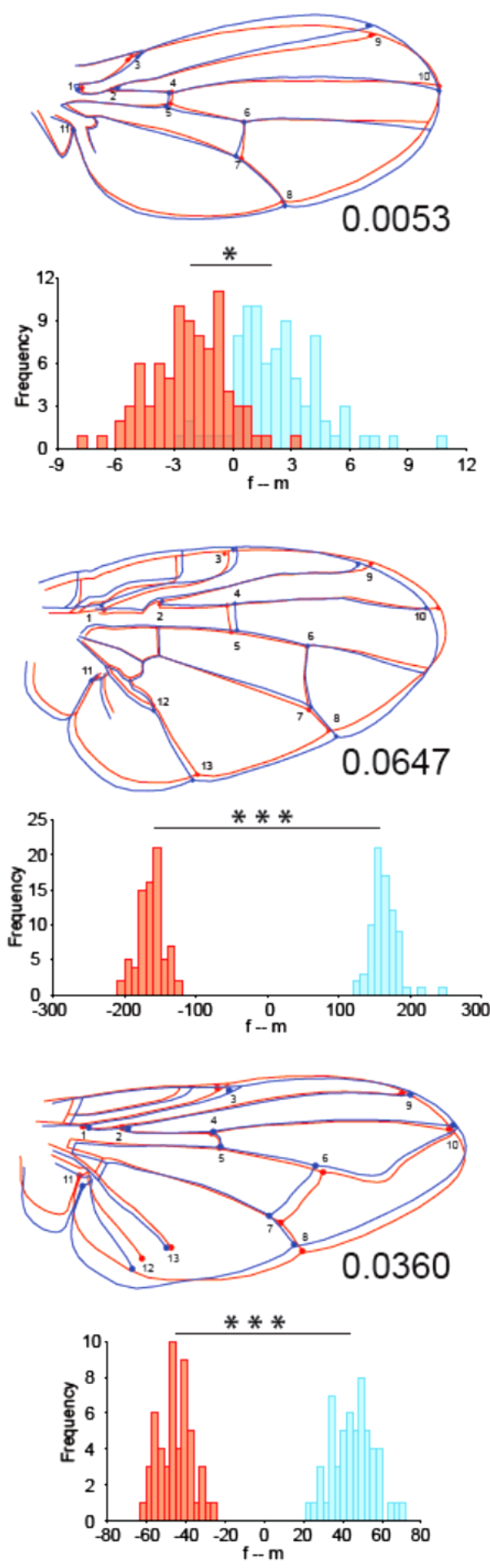


\subsubsection{Sexual dimorphism in wing shape}

Insects are known for a wide variety of sexual dimorphism, which includes among others differences in wing shape, the SShD (Pretorius 2005; Mitrovski-Bogdanović et al. 2009; de Camargo et al. 2015). In order to characterize the extent of SShD, I performed a DFA in Drosophila, Ceratitis and Musca flies. I found that male and female Drosophila wings were significantly different in shape, and both the allometric and non-allometric components contributed to this difference (Figure 5.3).

The total SShD of Drosophila wings was highly significant $(p<0.0001)$. After size correction, the DFA revealed less prominent but still significant difference in shape $(p=0.01)$ : male wings were broader than female wings, radial veins $R_{2+3}, R_{4+5}$ and M1 were more spread apart, but the length of the wing was not affected. Thus, the allometric component in the analysis of Drosophila wings introduced variation in the wing length resulting into female wings being shorter and more pointed. In contrast to Drosophila, the total SShD and non-allometric SShD were similar in Ceratitis and Musca suggesting that the allometric component contributed less in these two species. Similar to Drosophila, Ceratitis males had broader wings compared to females. Male wings were also shorter, mainly due to the contraction of the distal anterior region between landmarks 3-5, 9, and 10. The wing width of Ceratitis wings was increased along the whole wing edge, while Drosophila wings were more rounded in its distal part only and the proximal part did not show a large deviation. These results suggest that the huge SShD observed in Ceratitis can be mainly explained by the non-allometric component. In Musca, the SShD was the opposite to the other two flies: male wings were slightly longer and narrower than female with the anal part of the wing being highly enlarged.

Statistical significance of the observed SShD was also confirmed with Procrustes ANOVA (Table 5.4, effect: sex). Additionally, I found a considerable influence of environmental conditions on wing shape. Therefore, I split flies by sex into two groups and examined effects of temperature and density (for both $p<0.001$ ) on shape more closely to determine if there was any common pattern present. 
Table 5.4: Effects of sex, temperature and density on the wing shape scores, tested with Procrustes ANOVA. Df - degrees of freedom.

\begin{tabular}{|c|c|c|c|c|c|c|}
\hline Species & Effect & Sums of squares & Mean squares & Df & F & $p$-value \\
\hline \multirow{3}{*}{$\begin{array}{c}\text { Drosophila } \\
\text { melanogaster }\end{array}$} & sex & 0.00966851 & 0.0005371396 & 18 & 44.75 & $<.0001$ \\
\hline & temperature & 0.01727365 & 0.0009596474 & 18 & 79.96 & $<.0001$ \\
\hline & density & 0.00742772 & 0.0004126512 & 18 & 34.38 & $<.0001$ \\
\hline \multirow{3}{*}{$\begin{array}{l}\text { Ceratitis } \\
\text { capitata }\end{array}$} & sex & 0.15685566 & 0.0071298028 & 22 & 467.98 & $<.0001$ \\
\hline & temperature & 0.00340257 & 0.0001546623 & 22 & 10.15 & $<.0001$ \\
\hline & density & 0.00252489 & 0.0000573839 & 44 & 3.77 & $<.0001$ \\
\hline \multirow{3}{*}{$\begin{array}{c}\text { Musca } \\
\text { domestica }\end{array}$} & sex & 0.02733498 & 0.0012424991 & 22 & 33.41 & $<.0001$ \\
\hline & temperature & 0.00534071 & 0.0002427593 & 22 & 6.53 & $<.0001$ \\
\hline & density & 0.00298555 & 0.0000678534 & 44 & 1.82 & .0008 \\
\hline
\end{tabular}

\subsubsection{Sexual shape dimorphism in response to rearing temperatures}

To determine effects of deferent rearing temperatures in our flies, I first identified and removed the allometric component of shape variation. In each species, the DFA analysis clearly separated wings in two groups according to the rearing temperature (Figure 5.4, histograms).

In Drosophila and Ceratitis, the direction and strength of shape changes were similar in both sexes. Wings of flies grown at high temperature $\left(25^{\circ} \mathrm{C}\right.$ for Drosophila and $28^{\circ} \mathrm{C}$ for Ceratitis) were broader than wings of flies grown at $18^{\circ} \mathrm{C}$ (Figure 5.4, orange and blue wing outlines). The highest variation in width was observed in the proximal part of the wing in both species. Another observed effect was a shortening of wings with increasing temperature. In Ceratitis, temperature caused only minor changes, but in Drosophila the observed effect was strong. In contrast to these flies, shape changes in response to rearing temperatures varied between sexes in Musca. In female wings only minor shape changes were observed, while male wings were clearly narrower at the warm rearing temperature $(\mathrm{RT})$. 


\section{Males}
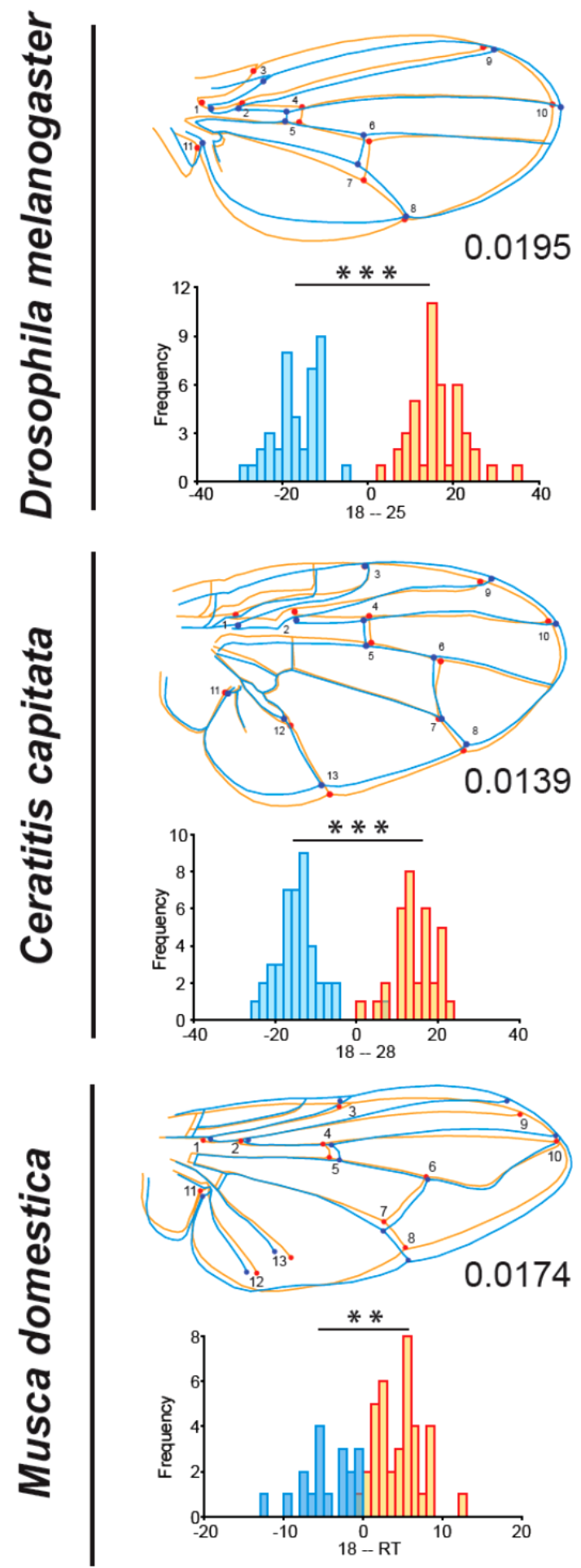

Females
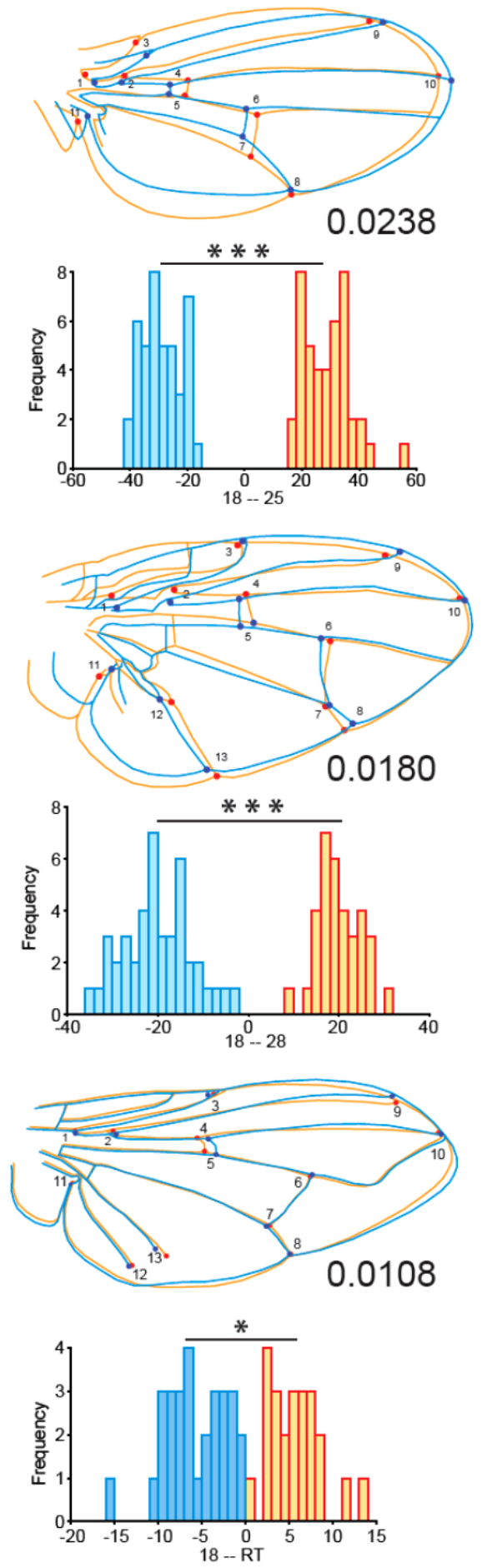

Figure 5.4: Changes of wing shape under conditions of different rearing temperatures. Wing shape alterations in response to temperature in D. melanogaster $(n$ (males $)=83 ; n$ (females $)=85)$, C. capitata $(n$ (males $)=81 ; n($ females $)=78)$, and $M$. domestica $(n$ (males $)=56 ; n($ females $)=51)$. Wing outlines represent differences between cold (blue) and warm (orange) temperatures with the scale factor 5 for each species. The magnitude of the shape variation is indicated in units of Procrustes distance with the corresponding p-values $\left({ }^{\star} p<0.05 ;{ }^{* *} p<0.001 ;{ }^{* *} p<0.0001\right)$. Histograms with the distribution of the discriminant scores show the shape separation into groups and significance of the changes. 
In addition to the overall shape changes, I also found displacements of the cross-veins. A shift of the $\mathrm{r}-\mathrm{m}$ crossvein, defined by the landmarks 4 and 5, was similar for all three species (Figure 5.4). Interestingly, this shift was the only significant shape alteration in response to different rearing temperatures in Musca females. Modifications of the bm-cu crossvein, defined by the landmarks 6 and 7, were found in different variations. In Drosophila wings, at the higher rearing temperature, the entire bm-cu crossvein was shifted towards the margin of the wing. In case of Ceratitis, the position of the landmark 7 did not change but the the landmark 6 marking the upper part of the vein shifted along the M1 vein. The bm-cu crossvein was not affected in Musca females but it was moved toward the center in the result of narrowing of the male wing.

\subsubsection{Sexual shape dimorphism in response to different larval densities}

Density is another powerful factor known to influence shape of insect wings (Bitner-Mathé and Klaczko 1999; Siomava et al. 2016). In order to characterize the impact of rearing density on wing shape, I grew flies in high and low densities. For Ceratitis and Musca, which are not well studied in this respect, I set up an intermediate density group. In the analysis, I first focused on the density extremes, expecting the most pronounced shape variation between them.

In Drosophila, the high rearing density resulted into elongated, narrowed, and more pointed male wings with $R_{2+3}, R_{4+5}$, and $M 1$ veins being close together (Figure 5.5A (m)). In contrast, Drosophila female wings were more rounded, mainly due to the widening of the distal part and a shift of $R_{2+3}$ and M1 veins apart (Figure 5.5A (f)). The r-m crossvein was displaced in both males and females, while the dm-cu crossvein was shifted in parallel with the r-m crossvein in females only. Statistically significant difference in wing shape of different density groups was confirmed with a permutation test for Procrustes distances run with the DFA ( $p_{\text {male }}=0.004$; $\left.p_{\text {female }}=0.01\right)$ and separate Procrustes ANOVA test $\left(p_{\text {male }}<0.0001 ; p_{\text {female }}<0.0001\right)$ (Figure 5.5A, discriminant scores). 


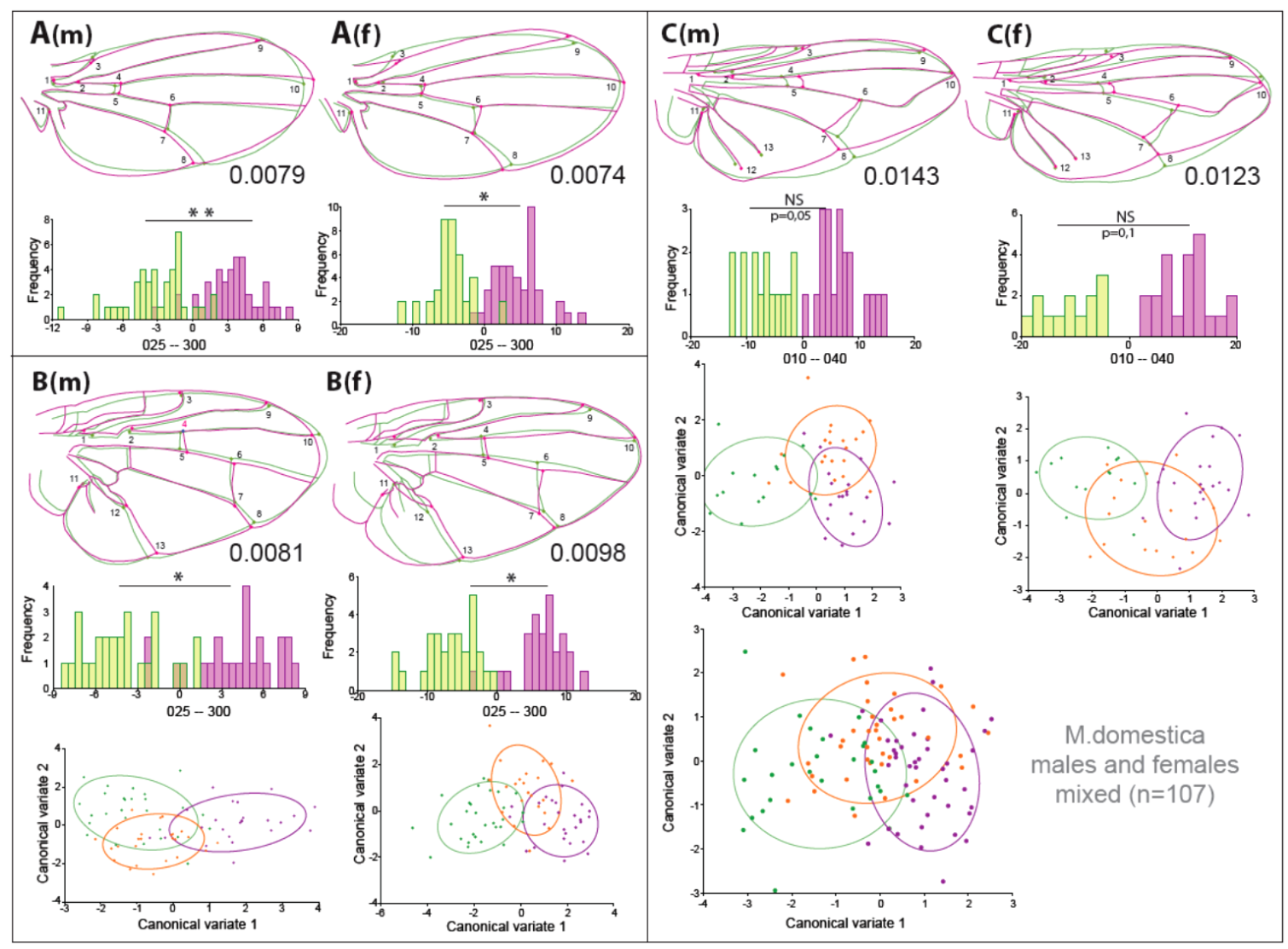

Figure 5.5 Wing shape changes in response to different larval densities. $D$. melanogaster males $(\mathrm{A}(\mathrm{m}))(n=83)$ and females $(\mathrm{A}(\mathrm{f}))(n=85)$, C. capitata males $(\mathrm{B}(\mathrm{m}))(n=51)$ and females $(\mathrm{B}(\mathrm{f}))(n=56)$, and M. domestica males $(\mathrm{C}(\mathrm{m}))(n=36)$ and females $\mathrm{C}(\mathrm{f}))(n=34)$. Wing outlines represent differences between the low (green) and high (violet) densities with the scale factor 9 for each species. The magnitude of the shape variation is indicated in units of Procrustes distance with the corresponding $p$-values $\left({ }^{*} p<0.05\right.$; $\left.{ }^{* *} p<0.001 ;{ }^{* *} p<0.0001\right)$. Histograms with the distribution of the discriminant scores show the shape separation into groups and significance of the changes. CVA scatter plots show distribution of different density groups and equal frequency ellipses with the probability 0.75 . The bottom right CVA scatter plot shows distribution of density groups for all Musca flies together $(n=107)$.

Male wings of Ceratitis displayed the opposite response to the high density than those of Drosophila. Wings were shorter and slightly broader with the maximum change in the proximal anterior region (Figure 5.5B $(\mathrm{m})$ ). The same region, together with the anal part of the wing, was enlarged in female wings, while the length was not effected (Figure 5.5B (f)). The r-m and dm-cu crossveins were displaced in a similar to Drosophila way. The observed changes were statistically significant for the density extremes in both sexes (DFA and CVA permutation tests for Procrustes distances: $p_{\text {male }} \leq 0.05$ and $p_{\text {female }} \leq 0.007$; Procrustes ANOVA $p_{\text {male }}<0.0001$ and $p_{\text {female }}<0.0001$ ) (Figure 5.5B, discriminant scores). Wings of flies that were raised at the intermediate density exhibited an intermediate shape (Figure 5.5B, CVA scatter plots). 
In Musca, the response to the high rearing density was the most similar between males and females. Wings of both sexes were narrower along the posterior wing margin (landmark 8), the $\mathrm{r}-\mathrm{m}$ and $\mathrm{dm}-\mathrm{cu}$ crossveins were shifted, and no variation in the wing length was found (Figure 5.5C). The landmark 3 and the whole $\mathrm{C}$ vein were shifted towards the wing center distinguishing females from males. Despite clear wing changes, the observed shape difference between the extreme rearing densities was not statistically significant in the DFA (permutation tests: $p_{\text {male }}=0.05$ and $p_{\text {female }}=0.1$ ) (Figure 5.5C, discriminant scores), while Procrustes ANOVA test clearly allowed to assign wings in two distinct groups ( $p_{\text {male }}=0.01$ and $p_{\text {female }}=0.02$ ). Similarly, permutation tests for CVAs gave contradicting results: $p$-values from permutation tests for Procrustes distances were statistically insignificant ( $p_{\text {male }}=0.05$ and $\left.p_{\text {female }} \leq 0.1\right)$, while $p$-values for Mahalanobis distances were highly significant for both sexes ( $p_{\text {male }}$ and $\left.p_{\text {female }}<0.0001\right)$. Because shape changes were similar between male and female flies, with the only exception of the landmark 3, I pooled all wings together to increase the sample size and ran a CVA for 107 flies of three densities (Figure 5.5C, bottom). This analysis showed significant difference between the highest and lowest densities according to the $p$-values for Procrustes and Mahalanobis distances $(p=0.03 ; p<0.0001)$ as well as for the Procrustes ANOVA test $(p<0.0001)$. The intermediate density group was allocated in-between the density extremes (Figure 5.5C, bottom: orange ellipse).

\subsection{Discussion and future work}

\subsubsection{Sexual shape dimorphism of Drosophila, Ceratitis, and Musca wings}

Besides interspecific differences in wing shape (Figure 5.1), I found a clear SShD in all three species (Figure 5.3). Variation in one of the wing shape parameters among Ceratitis females and males was in the similar range as the interspecific shape difference between Ceratitis and Musca (Figure 5.1, PC1) underpinning a strong sexual dimorphism that can mainly be explained by the non-allometric component of the shape variation (Figure 5.3). A more detailed analysis of the wing shape variation between sexes did not reveal any commonality among species.

In Drosophila, I observed a clear total SShD (Figure 5.2). Exclusion of the allometric component resulted into a less prominent but statistically significant difference. I found that male wings were more rounded with $R_{2+3}$ and $R_{4+5}$ veins were spread apart. This observation is in accordance with the previously published data (Bitner-Mathé and Klaczko 1999). However, a 
more detailed analysis based on landmarks performed here allowed me to characterize shape differences in more detail: the widening of the wing was mainly observed in the distal part, while the proximal part with vein intersects located there were very similar between sexes.

In Ceratitis differentiation between sexes mainly occurred due to variation in the width in the proximal and distal wing parts (Figure 5.1, PC1; Figure 5.3). I also found a significant elongation of female wings. This result supports the previously published data and confirms that male wings are shorter and wider than females wings (Churchill-Stanland et al. 1986).

In Musca, the narrowness seems to be more important to distinguish between sexes (Figure 5.1, PC2; Figure 5.3, non-allometric component). Females of wild Musca strains are known to have wings that are much larger than wings of males (Alves and Bélo 2002) and it fits in very closely with what I observed in the laboratory strain. Musca female wings were slightly wider in the distal region and the anal part of male wings was significantly enlarged in comparison to females, with the latter being the most noticeable shape difference between sexes.

\subsubsection{Sexual dimorphism in the size and shape relationships}

Variation in size is often known to entail changes in shape (Debat et al. 2003). Therefore, I tested whether a similar trend was present in wings of the three studied dipteran species. The comparison of growth trajectories among sexes supported presence of SShD in response to wing size changes in all three species (Figure 5.2, Table 5.2). In Drosophila, I found a clear contribution of the allometric component to the shape differences between males and females (Figure 5.3). For instance, a shift of $\mathrm{CuA} 1$ along the wing margin as described by B.C. Bitner-Mathé and L B Klaczko (1999) I could only detect when the allometric component was included. In general, exclusion of the allometric coefficient decreased the SShD significantly, suggesting that most of the observed shape differences resulted from the enlargement of female wings. Interestingly, in Ceratitis and Musca, I did not find a strong influence of the allometric component on wing shape. For example, variation in the wing length that was explained by the allometric component in Drosophila was solely explained by the non-allometric component in the other two species.

Overall, I found a clear sexual dimorphism in wing shape in all three species. The strong connection of the shape variation and wing size differences in Drosophila suggests that growth regulation, patterning and differentiation processes (e.g. vein placement and axis determination) during larval wing imaginal disc development are tightly linked. 


\subsubsection{Sexual shape dimorphism of wings in response to different environmental}

\section{conditions}

In 1999, B.C. Bitner-Mathé and L B Klaczko used a method of adjusted ellipses for the wing shape analysis in Drosophila and found neither displacement of radial veins nor rounding of wings in response to the rearing temperature. Based on these findings, they suggested that wing shape is more stable than wing size. In this study, I showed that despite radial veins were only minorly affected; there was a significant difference in wing shape of Drosophila flies grown at different temperatures (Figure 5.4). Previous studies demonstrated a stronger response of the distal wing parts to different rearing temperatures compared to the proximal parts (Debat et al. 2003). In contrast to these findings, I detected a high variation in the proximal landmarks (Figure 5.4, landmarks 1,3, and 11) and only mild changes in distal. One potential explanation for these discrepancies might be the range of rearing temperatures. While Debat at al. (2003) raised flies at stressful temperatures $\left(12^{\circ} \mathrm{C}\right.$ and $14^{\circ} \mathrm{C}$ as the cold temperature and up to $30^{\circ} \mathrm{C}$ for the high temperature), I used intermediate regimes $\left(18^{\circ} \mathrm{C}\right.$ and $\left.25^{\circ} \mathrm{C}\right)$. For instance, they showed the overall wing shape differences along the first two CVs. The variation in wing shape of flies raised at 12 $25^{\circ} \mathrm{C}$ was mainly explained by the first $\mathrm{CV}$. However, the second $\mathrm{CV}$ distinguishes wings of the extreme temperatures (i.e. $12^{\circ} \mathrm{C}$ vs. $30^{\circ} \mathrm{C}$ ) (Figure 5.6, Debat et al. 2003). It would be interesting to test what exact changes in the landmark configuration were explained by the first two CVs. Probably, the distal part of the wing is more responsive to stress conditions compared to the proximal part. In this case, my temperature regimes would not have covered these conditions.

In Ceratitis, I also found clear changes in the proximal part of the wing that were even more pronounced than in Drosophila (Figure 5.4, see landmarks 1 and 2). However, in Musca the proximal wing region was very stable at different rearing temperatures and variation was restricted to the distal part (Figure 5.4). I have previously shown that rearing conditions used in this analysis might not be optimal for the Italian Musca strain (see Chapter 4: Body size as an integral feature, Section 4.3.3), while rearing temperatures tested here for Ceratitis did not represent extreme values (Navarro-Campos et al. 2011). The observed wing shape changes in response to rearing temperatures might therefore be the result of the stress response in Musca similar to shape changes observed by Debat at al. (2003) for Drosophila (Figure 5.6). The more pronounced changes in proximal wing regions observed in Drosophila and Ceratitis in this study 
might be the actual response to temperature regimes within the normal and stress-free reaction norm.

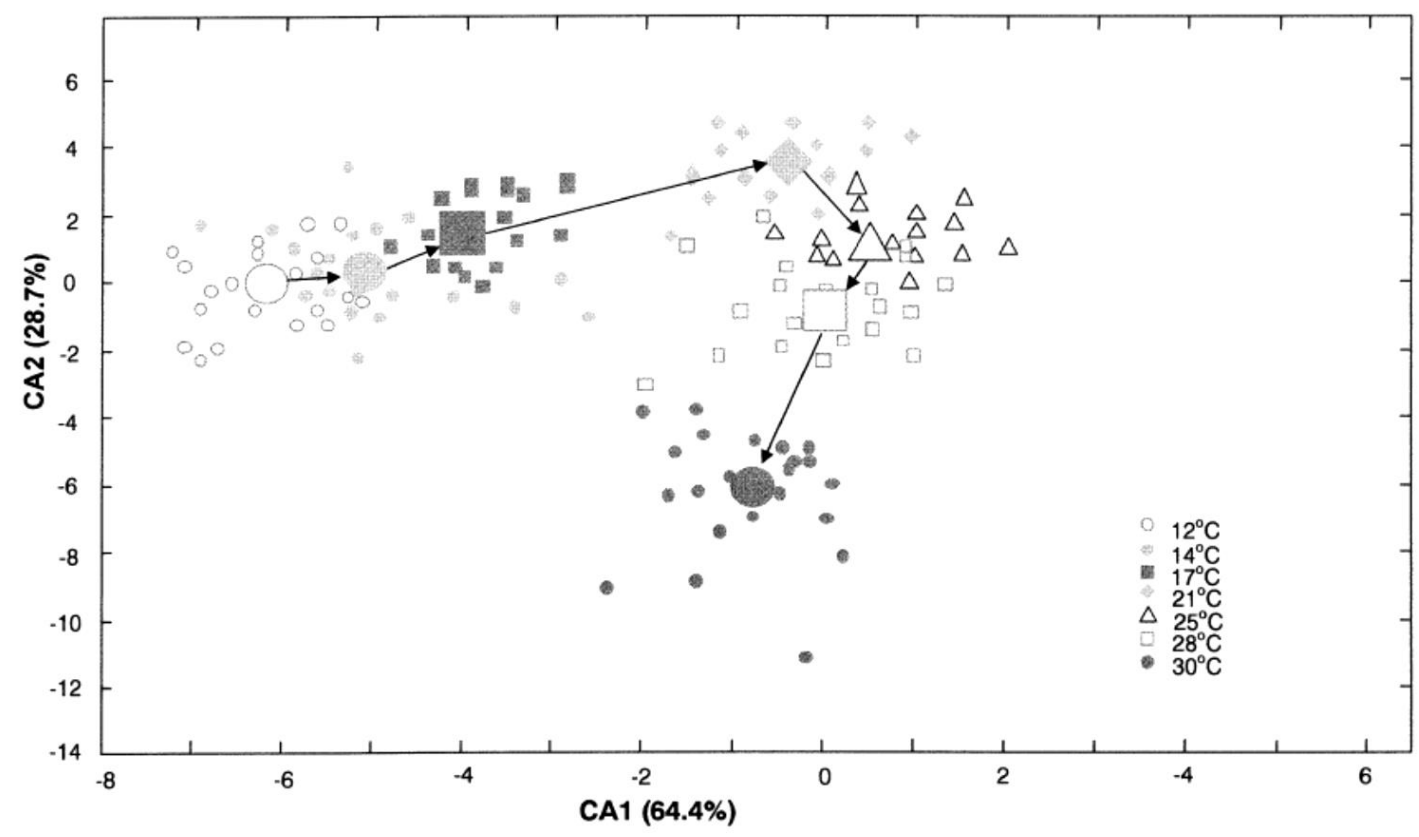

Figure 5.6: Analysis of wing overall shape variation (after Debat et al. 2003). First canonical plan computed from the canonical variates analysis on Procrustes coordinates (whole shape variation). Mean and individual values of the seven samples are shown. Arrows indicate the progression of growth temperature and show an overall curved trajectory.

In addition to changes in wing shape along the proximal-distal axis, shape analysis revealed a high variation in r-m (landmarks 4 and 5) and bm-cu (landmarks 6 and 7) crossveins that was common for all three species. Since these two veins develop somewhat later than radial veins (Marcus 2001) and they were significantly modified among temperatures and densities, they are likely to represent plastic features of the developing wing. It would be very interesting to compare the spatial and temporal expression of the $c d c 42$ gene and the Jun-N-terminal Kinase signal transduction pathway at different rearing conditions to relate the observed differences in placement of the r-m and bm-cu crossveins to the developmental plasticity.

\subsubsection{Potential functional implications of plasticity and sexual dimorphism in wing} size and shape for mating behavior

Wings and especially their surface area are known to play an important role in mating behavior in many dipteran fly species. Usually, male individuals produce species-specific courtship songs by fast and repetitive wing movements, flapping. For instance, the Caribbean fruit 
fly females of Anastrepha suspensa judge the size and vigor of a potential mating partner by the intensity of its courtship song (Burk and Webb 1983; Sivinski et al. 1984; Webb et al. 1984). Apparently, the intensity and audibility of these songs directly depend on the wing-beat frequency that the fly can afford considering energy costs and fragility of wings.

From Figure 5.1, I previously deduced that Musca have wings that are longer and narrower that those of Ceratitis and Drosophila (PC2). This means that Musca have the highest wing span (b) among the studied flies. It is known that the wing span is proportional to the moment of inertia (I): $I \sim b^{2}$ (Shyy et al. 2013). Thus, the wing moment of inertia is also the highest in Musca. Taking into account that the moment of inertia is inverse to the wing-beat frequency $(f)\left(f \sim \frac{1}{\sqrt[8]{I}}\right)$ (Shyy et al. 2013), I conclude that the wing-beat frequency is the lowest in Musca in comparison with the other two flies and, therefore, their wings are less suited for buzzing.

Additionally, male wings of this species were more pointed and even slightly elongated compared to female wings, additionally increasing the moment of inertia and required inertial power. In support of my hypothesis, there is a fact that Musca flies do not have a mating behavior based on a courtship song. It has been shown that the mating process in house flies is in many cases initiated during flight by an attack ("mating strike") of a male against the back or side of a female. A successful "mating strike" usually results into the immediate landing and start of the copulation process (Murvosh et al. 1964). My finding suggests that there is probably no adaptive advantage of the frequent flapping in these flies but their wing shape might be more under selection to improve the flight performance, what is facilitated by long and narrow wings (Alves and Bélo 2002).

In contrast to the house fly, mating behavior is present in Drosophila and Ceratitis. Usually, their females favor males with a higher audibility (Churchill-Stanland et al. 1986; Partridge et al. 1987). This study revealed that male wings were wider than female wings, shorter in case of Ceratitis, and radial veins were more spread apart making wings more compact. The allometric component of shape additionally increased this difference in Drosophila. The short, wide and rounded wings of males of these species are likely to displace more air and repeat calling song pulses more quickly than long narrow wings, and the wing moment of inertia seems to be low enough to buzz. However, I still observed a strong variation in their wing shape along the PC1 (Figure 5.1), which can be explained by the frequency of mating songs. 
These flies produce two different types of wing vibration during the pre-mount courtship: the pulse song and the sine song (Bennet-Clark et al. 1976; Rolli 1976; Webb et al. 1983; Briceño et al. 1996; Briceño and Eberhard. 2002;). The sine song is a continuous sinusoidal humming generated by small amplitude wing vibrations (Spieth 1952; Ewing and Bennet-Clark 1968; Hall 1994; Ejima and Griffith 2007; Markow and O’Grady 2005). In Drosophila, its frequency is ranging from $110-185 \mathrm{~Hz}$ (Wheeler et al. 1988), with the median value of approx. $160 \mathrm{~Hz}$ (Wheeler et al. 1988) or sometimes even $130 \mathrm{~Hz}$ (Talyn and Dowse 2004). In Ceratitis, this frequency is similar to Drosophila, $165 \mathrm{~Hz}$ (Webb et al. 1983). On the other hand, there is a difference in the pulse songs between these species. In case of Drosophila, it is series of single pulses (one to three cycles) separated with interpulse intervals. The frequency of these pulses is between 200-280 Hz with the median value of $240 \mathrm{~Hz}$ (Cowling and Burnet 1981; Rybak et al. 2002). Instead of pulses, Ceratitis use a continuous vibration of wings, while a male is looking towards a female and keeping its abdomen bent ventrally (Briceño et al. 2002). An average frequency of such buzzing is about $350 \mathrm{~Hz}$ (Webb et al. 1983). Thus, the frequency of Ceratitis buzzing is almost $70 \%$ higher than the Drosophila one. Therefore, Ceratitis should have a lower moment of inertia. This assumption is supported by the fact that the moment of inertia is smaller when the wing mass is concentrated near the axis of rotation (Berg and Rayner 1995). In Ceratitis, I observed wings that were wider in the proximal part, while in Drosophila this part was the narrowest (Figure 5.1, PC1 axis). Thus, I assume that the major shape difference observed in Figure 5.1 might be tied with the mating behavior and beating frequency of the flies.

A further support for my hypothesis comes from the observed variation in wing shape in response to rearing conditions. I found rounded and shortened wings in Drosophila and Ceratitis flies grown at warm temperature, which normally stimulates reproduction. In contrast, Musca wings showed the opposite trend: they were narrower at higher rearing temperature in accordance with the lack of the courtship song behavior. A similar trend was observed for different rearing densities. In conditions of a strong competition as a consequence of crowding, I found wide and short wings in Ceratitis, suggesting that males might produce more attractive songs for females. In Musca, crowded conditions, similar to warm temperature, resulted into narrow male wings, supporting the idea that no selective pressure towards short and wide wings is present. Drosophila males showed an enlargement in the anal part of the wing, which fits to our hypothesis. Surprisingly, the wing was also longer in these conditions. This could be due to the chosen rearing 
densities for Drosophila that might not represent a stress situation. Another explanation could be the remaining beating frequency. As it was shown by Ray et al. (2016), biological organisms are designed with a margin of safety. Thus, even though wings became longer and the moment of inertia increased, the fly could possibly afford the same beating frequency.

In this part of my study, I found that that there was not only a clear SShD in the three dipteran flies but also a sex specific wing shape alteration in response to different rearing conditions. The three fly species of the same order exhibit significant differences and a clear variation in wing shape and represent an interesting system to further study the impact of these differences on potentially adaptive traits such as flight performance and mating behavior. 


\section{GENE EXPRESSION IN FLY EMBRYOS AND WING IMAGINAL DISCS}

In the last three decades of the $20^{\text {th }}$ century, it was shown that development of the Drosophila wing is a complex process, which requires a large number of genes (reviewed in Klein 2001; Morata 2001; Dahmann et al. 2011). These genes form an intricate network that becomes even more complicated with the discovery of new genes that may contribute to development (Butler 2003). By now, most of knowledge about the wing formation and genes involved in this process was obtained from the Drosophila model. This makes it difficult to judge about the similarity of the network and processes between different insect species.

Thus, to look at the resemblance of the wing development in D. melanogaster, C. capitata, and $M$. domestica, I compared expressions of homologous wing patterning genes. For the comparison, I used nine genes that are known to be involved in development and patterning of wings. They are well studied in Drosophila but their expression has not been described in the other two species, yet. Three of the chosen genes are responsible for the formation of the AP compartment boundary (engrailed (en), hedgehog $(h h)$, and decapentaplegic (dpp)), two genes establish the dorsoventral (DV) compartment boundary (wingless (wg) and apterous (ap)), and the last four genes are involved in wing patterning (daughters against Dpp (dad), optomotor blind $(o m b)$, spalt major (salm), and knirps $(k n i))$. In this chapter, I present expression patterns obtained for the above mentioned genes. I describe similarities and differences between them and discuss possible changes in gene interactions in the three species in Section 6.3. The results described here will be brought to a close and submitted as a manuscript to Development Genes and Evolution Journal in co-authorship with Dr. Ernst A Wimmer.

\subsection{Gene regulation in the developing fly wing}

Holometabolous insects, i.e. flies, bees, butterflies and others, have a four-stage life cycle during which they undergo the complete metamorphosis from an embryo to an adult via a number of larval and pupal stages. The complete metamorphism and presence of intermediate stages, such as larva and pupa, is the key feature that distinguishes species of the superorder Endopterygota from other insects. Many crucial morphological changes occur at these stages. For example, during the pupal transformation, structures of the adult flies or butterflies will be developed from imaginal discs, which are small clusters of cells set aside from the larval epidermis during embryogenesis and which proliferate and differentiate during the larval life (Postlethwait 
1978; Garcia-Bellido and de Celis 1992; Cohen 1996). One of the adult structures formed during the metamorphosis is the wing. It is developed from the wing imaginal disc that includes tissue giving rise to the wing hinge and thorax (Figure 6.1) (reviewed in Lewis and Held 2005).

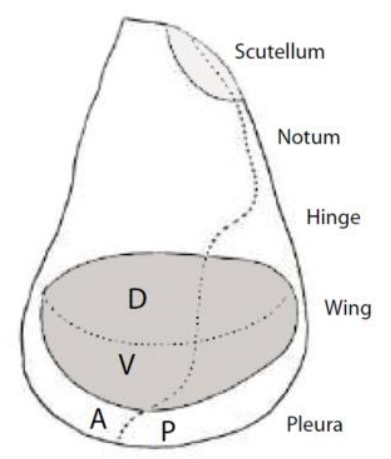

Figure 6.1: Fate map of the Drosophila wing imaginal disc. The AP and DV boundaries are shown with dashed lines. D indicates the dorsal compartment of the disc, $\mathrm{V}$ - ventral, A - anterior, $\mathrm{P}$ - posterior. Wing pouch and scutellum are shaded. (modified after Bryant 1975)

A complex regulation of developmental processes is required during the maturation of the disc to control and guide differentiation of the growing tissue. This regulation includes multilevel gene networks that not only control the wing development but also connect other body structures to coordinate development of the entire animal. Some of the genes that are involved in the wing development are already expressed at embryonic stages, e.g. en (DiNardo et al. 1985; Kornberg et al. 1985). Together with $w g$, en determines the proper position of wing imaginal discs in D. melanogaster (Couso et al. 1993). It also causes the first specialization of wing discs, since 10 to 13 cells of the posterior wing primordium continue expressing en after separation, while the other 12 to 15 cells do not(; Lawrence and Morata 1976; Kornberg et al. 1985). During development, cells of the wing imaginal disc proliferate, the disc grows, and the expression pattern of en expands occupying the entire posterior compartment (Kornberg et al. 1985). One of the main functions of en in this compartment is to define the anteroposterior boundary and activate a cascade of downstream genes. Consequently, presence of en in the posterior compartment promotes expression of $h$ in the same cells (Lee et al. 1992; Tabata et al. 1992). In Drosophila, the expression pattern of $h$ coincides precisely with that of $e n$. hh encodes a secreted protein that induces expression of $d p p$ (Basler and Struhl 1994; Zecca et al. 1995), which is the key gene of this part of the study. Another key gene should have been dad. The expression of dad is positively regulated by Dpp, which is sufficient for activation of the dad expression in the developing wing imaginal disc (Tsuneizumi et al. 1997). The expression pattern of $d a d$ reflects expression of $d p p$ but has a wider stripe that straddles the AP compartment boundary of the disc. In contrast to $d p p$, 
dad is expressed in cells of both the anterior and posterior compartments. Altogether the three mentioned above genes (en, $h h$, and $d p p$ ) establish the AP compartment boundary in the wing disc with cells that do not mix together and may express compartment specific and common genes (e.g., dad).

The DV compartment boundary appears later in development (Garcia-Bellido et al. 1976). Formation of the dorsal and ventral compartments mainly depends on the two genes and their products: $w g$ and $a p$. For the first time, expression of $w g$ can be detected in wing imaginal discs in the second instar (Couso et al. 1993). In Drosophila, expression of this gene undergoes dynamic changes during development. At the beginning of the second instar, $w g$ is initially expressed in the ventral region of the wing disc and it specifies the wing field. As development proceeds, wg occupies the whole distal region with a stripe of a high concentration at the presumptive wing margin. In the late third instar, $w g$ is expressed in a few cells along the wing margin, in a double ring around the distal region of the wing, and in a wide stripe in the notum. During the late second instar, wg plays a leading role in positioning of the ap expression. Expression patterns of the two genes overlap ventrally (Williams et al. 1993; Ng et al. 1996), while later ap mark cells that belong to the dorsal compartment of the wing (Cohen et al. 1992; Diaz-Benjumea and Cohen 1993; Williams et al. 1993) and establishes the proper DV boundary via genes such as scalloped, vestigial, fringe, etc (Williams et al. 1993; Irvine and Wieschaus 1994).

When all boundaries are defined and four compartments of the wing imaginal disc are specified, other genes that drive wing specification are required. For example, it was shown that salm together with $\mathrm{kni}$ are responsible for the formation of the longitudinal veins in Drosophila (Lunde et al. 1998; Lunde et al. 2003; Crozatier et al. 2004). Expression of salm is restricted to the wing pouch in the developing wing disc where it forms a broad central domain that covers cells from the $\mathrm{R}_{2+3}$ provein until the anterior limit of the $\mathrm{CuA}_{1}$ provein (Nellen et al. 1996; Barrio and de Celis 2004). The product of the gene is a zinc-finger transcription factor (Spalt) that contributes to the positioning of the longitudinal veins via inhibition of iroquois ( $\mathrm{R}_{4+5}$ vein) and regulation of the gene complex kni and knirps-related in the concentration dependant manner $\left(\mathrm{R}_{2+3}\right.$ vein) (reviewed in Crozatier et al. 2004). Spalt is also indirectly involved in the development of the $\mathrm{M}$ vein and regulates the omb gene to position the $\mathrm{CuA}_{1}$ vein. Interaction of these and many more genes results in a rather simple pattern of five longitudinal veins and two cross-veins in an adult Drosophila wing. The vein pattern is an important characteristic of wings in every species, 
because it provides rigidity of wings that is required for the powered flight (Combes and Daniel 2003; Wootton et al. 2003) and distributes sensory organs required for the coordination of the wing motion (Kammer 1985).

\subsubsection{Identification of homologous wing patterning genes in Drosophila, Ceratitis,}

\section{and Musca}

To obtain sequences of homologous proteins in Ceratitis, I performed BLASTP search with the known Drosophila protein sequences at the NCBI, web site: http://www.ncbi.nlm.nih.gov/. The found Ceratitis proteins were derived from genomic sequences annotated using the gene prediction method Gnomon, which is supported by expressed sequence tag evidence, and predicted by an automated computational analysis. Some of the found proteins showed a high similarity (e.g. En 88\%, Ap 79\%) between species, while others were quite different (e.g. Kni 36\%). The full list of found homologs and the level of their similarity to Drosophila sequences can be found in Appendix 11.4, Table 11.1 in there. A number of genes were predicted to have more than one isoform (e.g. $d p p$ isoform X1 and $d p p$ isoform X2) (Figure 6.1).

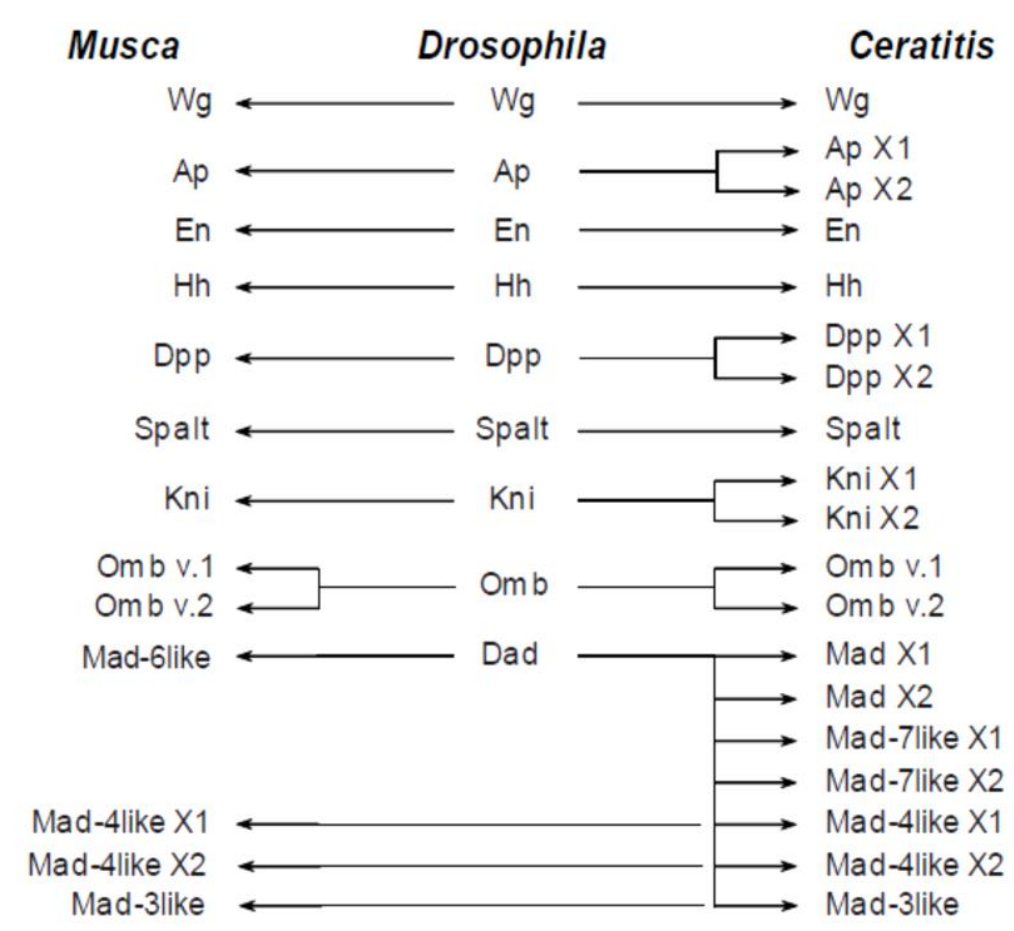

Figure 6.1: Predicted homologs of Drosophila proteins in Ceratitis and Musca. Known Drosophila genes that were used for the BLAST search are listed in the middle. Found homologs in Ceratitis and Musca are shown with the respective isoforms marked as $\mathrm{X} 1$ or $\mathrm{X} 2$. 
For the Omb protein, I initially found two corresponding proteins in Ceratitis (Figure 6.1). These two proteins had a good match with the Drosophila protein in a way that each of the predicted Ceratitis sequences nicely aligned to one of the two halves of the Drosophila Omb (Figure 6.2).

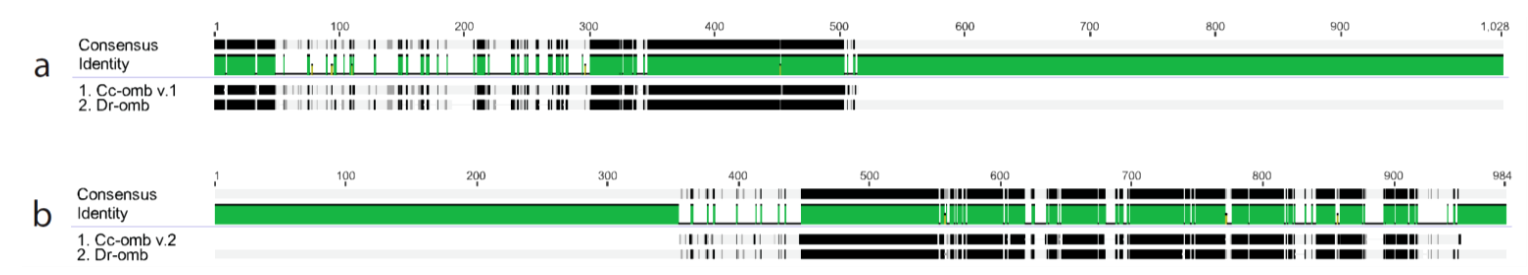

Figure 6.2: Alignment of the Omb proteins from Drosophila and Ceratitis. Alignment of Drosophila Omb with Ceratitis Omb v.1 (a) and Omb v.2 (b). Dr - Drosophila melanogaster, Cc - Ceratitis capitata. Black boxes show identical amino acids (AA) in the overlapping regions, gray boxes - different AA, green boxes include both identical and non-overlapping regions of the aligned proteins, and numbers refer to the length of the consensus sequence.

On the gene level, these two proteins were predicted to be two independent and nonoverlapping genes. I named these genes and respective proteins as omb v.1 (protein: Omb v.1) and omb v.2 (protein: Omb v.2); both of them were included in the further analysis. The mentioned above BLAST search was performed in 2013, shortly after the release of the Ceratitis genome (version: 14-MAY-2013). Later, however, some of the found genes underwent some modifications and even re-annotations. Thus, in the updated version released on 13-APR-2015, genes omb v.1 and $o m b v .2$ were united into one omb gene and the old reference sequences were removed in result of the standard genome annotation processing. The new omb was predicted to have three alternative transcripts, but none of them was similar to short versions of the old annotation (Figure 6.3).

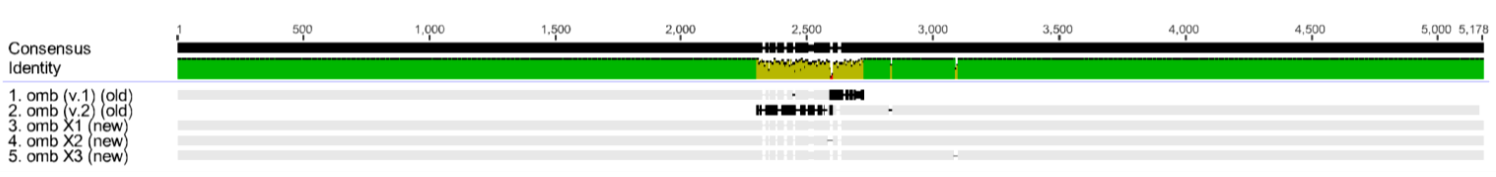

Figure 6.3: Alignment of $o m b v .1$ and $o m b$ v.2 (Prediction 2013) with omb (Prediction 2015) in Ceratitis. Old refers to the prediction 2013, new - prediction 2015. Different RNA isoforms of the new prediction are marked as X1, X2, and X3. Gray boxes show identical bp in the overlapping regions, black boxes - different $b p$, and numbers refer to the length of the consensus sequence. 
In the same release, 13-APR-2015, the previous annotation of the gene salm was also changed due to more evidence. The newly annotated salm gene is now located downstream of the previously annotated salm and neighbors it (Figure 6.4). For this study, I amplified and tested both predicted genes.

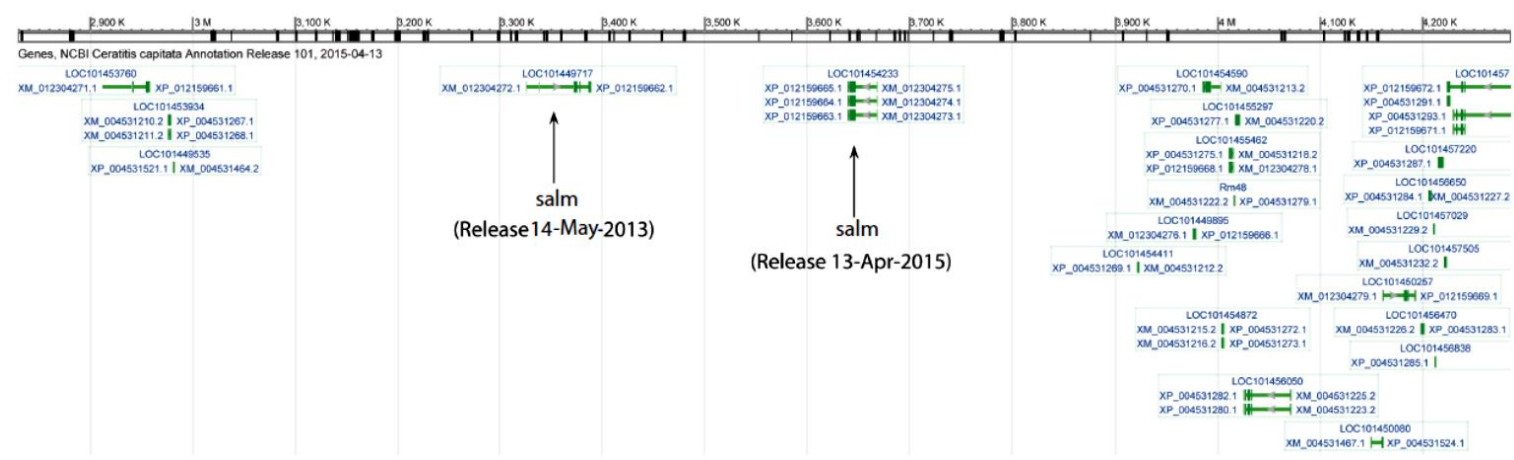

Figure 6.4: Newly annotated salm is located downstream the previously annotated salm. Ccap_1.0 Scaffold5 (annotation release 101, 13-Apr-2015) with RNA isoforms coding proteins. Both predictions of salm are marked with arrows and the respective annotation release.

With Dad, I could not find a corresponding prediction in Ceratitis. All found proteins and the respective genes were annotated as Mother against decapentaplegic (Mad) with the similarity from $29 \%$ to $34 \%$ and the query cover $29 \%$ to $99 \%$ with the Drosophila Dad (Appendix 11.4, Table 11.7 in there). All found variants of mad in Ceratitis (Figure 6.1) were amplified in the following RACE-PCR and checked with ISH. All results obtained with BLASTP were also confirmed with TBLASTN search.

Since the Musca genome was not available at the time when I performed the BLAST search, I used another approach to identify sequences of the homologous proteins and genes in these species. For this, I created a transcriptome database in Geneious ${ }^{\circledR}$ software version 8.1.5 using sequences kindly provided by Dr. Daniel Bopp and his collaborators (University of Zürich, Institute of Molecular Life Sciences, Switzerland). This database was then used for TBLASTN search with the known Drosophila and recently found Ceratitis proteins. With this approach, I was able to identify all genes or at least partial sequences of them except of ap. Later, when the Musca genome became available at NCBI, I confirmed the previously found Musca genes and identified the missing ap. Similar to Ceratitis omb, I found two versions of this gene in Musca (Figure 6.1, 6.5, and Appendix 11.4, Table 11.7 in there). They were named in the same way (omb v.1 and omb v.2) and both of them were included in the analysis. 
a

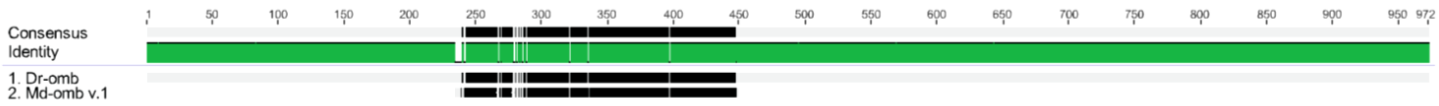

b

\begin{tabular}{l}
$\begin{array}{l}\text { Consensus } \\
\text { Identty }\end{array}$ \\
1. Dr-omb \\
2. Md-omb \\
\hline
\end{tabular}

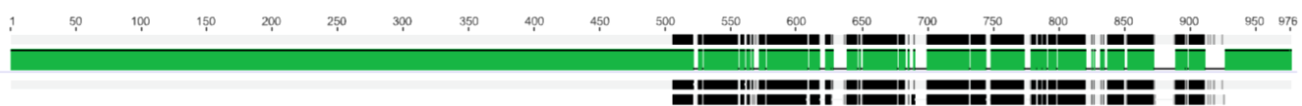

Figure 6.5: Alignment of the Omb proteins from Drosophila and Musca. Alignment of Drosophila Omb with Musca Omb v.1 (a) and Omb v.2 (b). Dr - Drosophila melanogaster, Md - Musca domestica. Black boxes show identical AA in the overlapping regions, gray boxes - different AA, green boxes include both identical and non-overlapping regions of the aligned proteins, and numbers refer to the length of the consensus sequence.

Searching for dad, I used the known Drosophila Dad and all available Mad proteins found in Ceratitis. In result, I identified three possible homologs: mad, mad-3like, and mad-4like. Later, the list was expanded with mad-6like, found in the database at the NCBI. On the phylogenetic tree, mad-6like fell together in a group with dad from Drosophila and Tribolium as well as with mad-7like from Ceratitis (Figure 6.6).

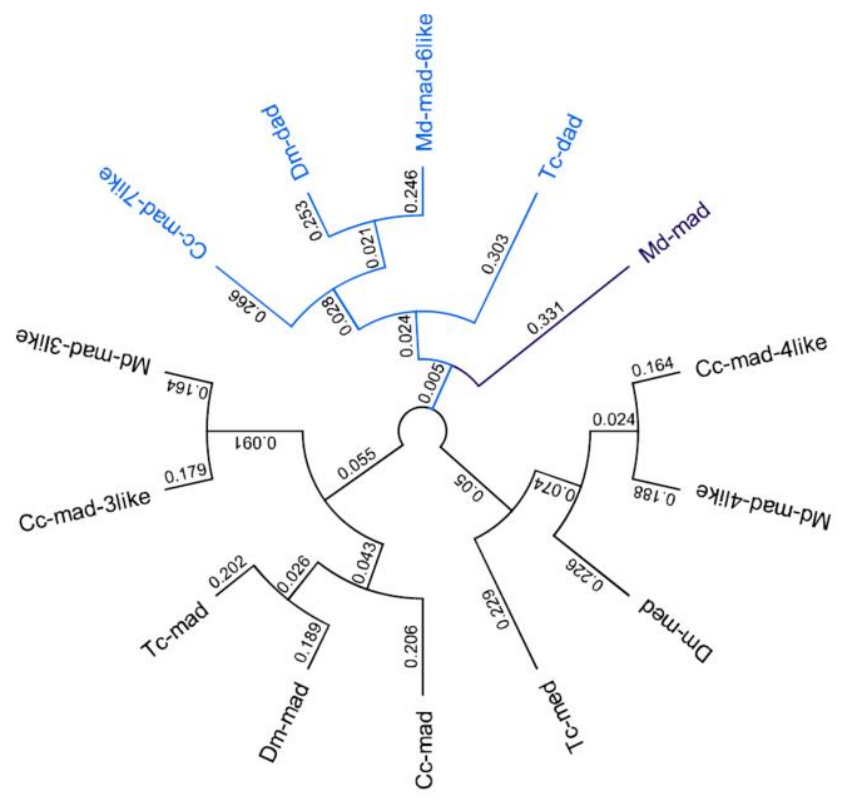

Figure 6.6: Phylogenetic tree of $\mathbf{d a d}$ and $\mathbf{m a d}$ genes. The phylogenetic tree was generated in Geneious version 8.1.5 using Geneious Tree Builder and the neighbour joining method. The tree is shown as cladogram. Cc - Ceratitis capitata, Dm - Drosophila melanogaster, Md - Musca domestica, Tc Tribolium castaneum. Branch that includes Drosophila and Tribolium dad genes is highlighted with blue. 


\subsubsection{Verification of predicted RNA isoforms in Ceratitis}

To verify 3'- and 5'-UTRs of the predicted Ceratitis genes, I created RACE-ready cDNA libraries (see Chapter 3: Materials and Methods, Section 3.4.3) from embryonic poly(A) mRNA. These libraries were used to amplify UTRs with specific primers (see Chapter 3: Materials and Methods, Section 3.4.1; Appendix 11.3, Table 11.2 in there) and the universal primer mix provided along with the kit (Appendix 11.3, Table 11.2 in there). Amplified fragments were cloned into the pCR II vector (see Chapter 3: Materials and Methods, Section 3.4.4) and sequenced (see Chapter 3: Materials and Methods, Section 3.4.5). Further analysis of sequences from Ceratitis revealed both single nucleotide polymorphisms (SNPs) and major changes in the UTRs and/or coding DNA sequence (CDS) (Figure 6.7 and Appendix 11.3, List 11.1 in there).

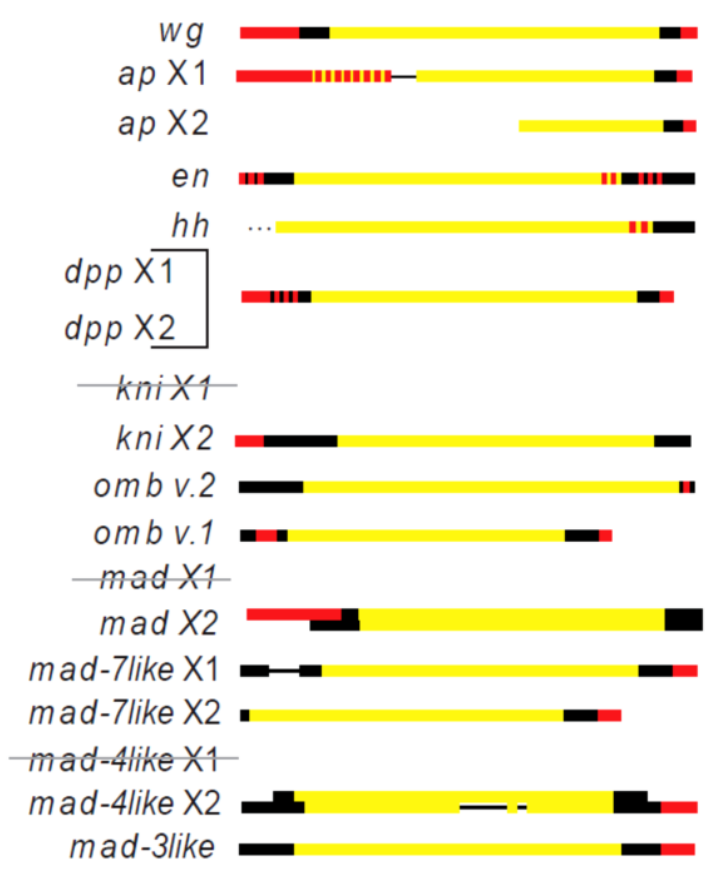

Figure 6.7: RNA isoforms of analyzed genes in Ceratitis. List of RNA isoforms with a scheme of sequences: black solid line - UTR, yellow line - CDS, red line - differences found, black thin line -intron. Struck-through isoforms were not found, dots - structure is unknown.

en: SNPs in both UTRs, SNPs in CDS;

$h h$ : SNPs in CDS;

$d p p$ : one RNA isoform, major differences in both UTRs;

wg: major differences in both UTRs;

ap: major differences in the 5'-UTR and CDS significantly effecting the corresponding protein, an intron was detected; 
mad: RNA isoform X1 was not found, major differences in the 5'-UTR of the RNA isoform $\mathrm{X} 2$, additional RNA isoform $\mathrm{X} 3$ was found with CDS of the isoform $\mathrm{X} 2$; mad-3like: major differences in the 3'-UTR; mad-4like: RNA isoform X1 was not found, major differences in both UTRs, additional RNA isoform X3 was found with major differences in CDS significantly effecting the protein;

mad-7like: major differences in both UTRs, an intron was detected in the RNA isoformX1; omb v.1: SNPs in the 3'-UTR;

omb v.2: major differences in both UTRs;

kni: RNA isoform X1 was not found, major differences in the 5'-UTR;

Even though genes omb v.1 and omb v.2 were united into one omb gene in the new annotation (13-APR-2015), my RACE-PCR data supported the previous annotation showing that there were at least two different 3'-UTRs present in our flies. Further analysis of the region of unification with specific primers (Appendix 11.3, Table 11.3 in there) confirmed presence of two variants of $o m b$ in Ceratitis: the full-length $o m b \mathrm{X} 1$ and a short version omb v.1.

\subsubsection{Verification of predicted RNA isoforms in Musca}

To verify 3'- and 5'-UTRs of the found Musca genes, I created RACE-ready cDNA libraries from embryonic poly(A) mRNA in a similar to Ceratitis way, amplified fragments, cloned and sequenced them (see Chapter 3: Materials and Methods, Section 3.4). I was not able to amplify UTRs of some genes, but the obtained results were sufficient to confirm presence or absence of predicted RNA isoforms. If none of the UTRs was amplified in RACE-PCR, I designed gene specific primers targeting CDS. These fragments were cloned, sequenced (see Chapter 3: Materials and Methods, Section 3.4), and used as templates for ISH probes (see Chapter 3: Materials and Methods, Section 3.5.2). Analysis of the obtained sequences showed that there were present SNPs as well as major changes in CDS and the UTRs (Figure 6.8). Also, my RACE-PCR data did not support the hypothesis that there are two different omb genes in Musca because I was able to identify neither 3'-UTR of omb v.1 nor 5'-UTR of omb v.2. 


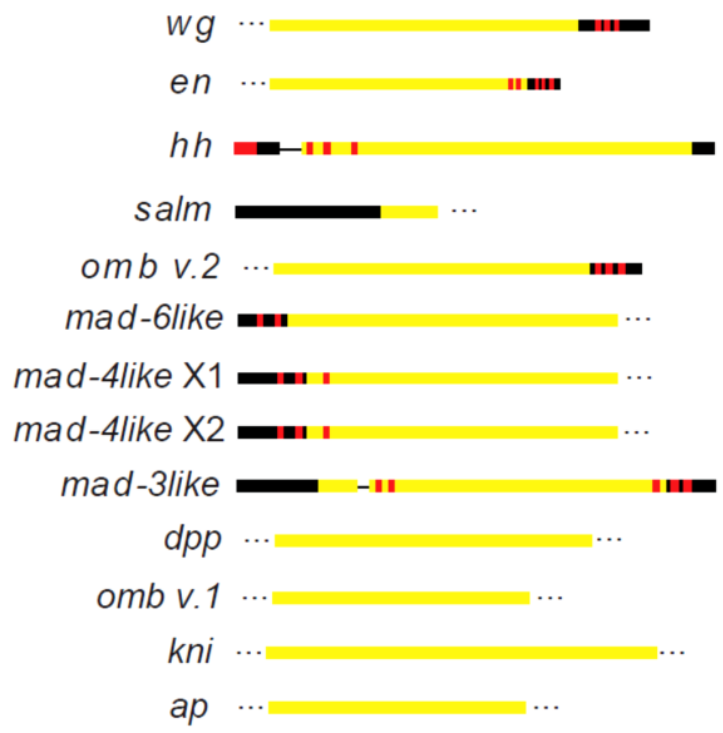

Figure 6.8: RNA isoforms of analyzed genes in Musca. List of RNA isoforms with a scheme of sequences: black solid line - UTR, yellow line - CDS, red line - difference found, black thin line - intron, dots - structure is unknown.

en: SNPs in the 5'-UTR, minor differences in CDS;

hh: major differences in the 5'-UTR, minor differences in CDS, an intron was detected; $d p p$ : only a CDS fragment was available;

wg: SNPs in the 3'-UTRs;

ap: only a CDS fragment was available;

mad-3like: SNPs in the 3'-UTR, SNPs in CDS, an intron was detected; mad-4like: SNPs in the 5'-UTRs of both isoforms, SNPs in CDS;

mad-6like: SNPs in the 5'-UTR;

omb v.1: only a CDS fragment was available;

omb v.2: SNPs in the 3'-UTR;

salm: no difference was found;

kni: only a CDS fragment was available; 


\subsection{Expression of genes involved in the wing development and patterning}

\subsubsection{Gene expression in wing imaginal discs of Ceratitis and Musca}

To make sure that the molecular mechanisms and gene networks are conserved between the species, I performed ISH on wing imaginal discs that were dissected of the L3 instar and fixed with 4\% PFA (see Chapter 3: Materials and Methods, Section 3.5.1).

The following three genes, $e n, h h$, and $d p p$, are known to establish the AP boundary in wing imaginal discs in Drosophila. I checked expression of these genes in Ceratitis and Musca and found that their patterns looked similar in Ceratitis, while in Musca I detected some variation. In Ceratitis, en was expressed in the posterior part of the disc in all three morphological regions: the wing pouch, hinge and notum (Figure 6.9A). The expression in the notum, however, was slightly different from the known Drosophila expression: there was no abrupt turn posteriorly before the presumptive notal region. The border was defined by a fairly straight line that went to the margin of the disc and proceeded as a thin strip of stained tissue along the posterior margin between the disc stalk and bulk of the posterior compartment (Figure 6.9A, arrowhead).

Ventral to the notum, the boundary of $h h$ followed the border of en (Figure 6.9A and B), while the staining of the very dorsal thin stripe of tissue present in the en staining was not found in the expression pattern of $h$ h (Figure 6.9B, arrowhead). Surprisingly, $d p p$ reflected the expression pattern of en, not $h$. It was a straight line across the folds of the wing pouch and presumable hinge, straight line to the posterior margin of the disc, and narrow strip going to the disc stalk (Figure 6.9C).

As expected, I could detect expression patterns of both en and $h h$ in the posterior compartment of the wing imaginal disc in Musca (Figure 6.9D and E). Staining of en revealed a clear boundary between the anterior and posterior compartments in the wing pouch region (Figure 6.9D). A faint staining was also found in the hinge region but it was absent in the notum (Figure 6.9D, arrowhead). In most cases, $h h$ staining was so faint that it was difficult to define a precise AP boundary (Figure 6.9E). Expression of $d p p$ was very variable. In some discs, I could only detect it in the wing pouch (Figure 6.9F), while no expression was found in the presumable hinge and notum (Figure 6.9F, arrowhead). In a number of discs, the staining was also present in the hinge region (Figure 6.9F', arrowhead) and even in the ventral notum (Figure 6.9F", arrowhead). However, I never detected the $d p p$ expression in the thin strip of tissue going to the disc stalk along the posterior margin. 

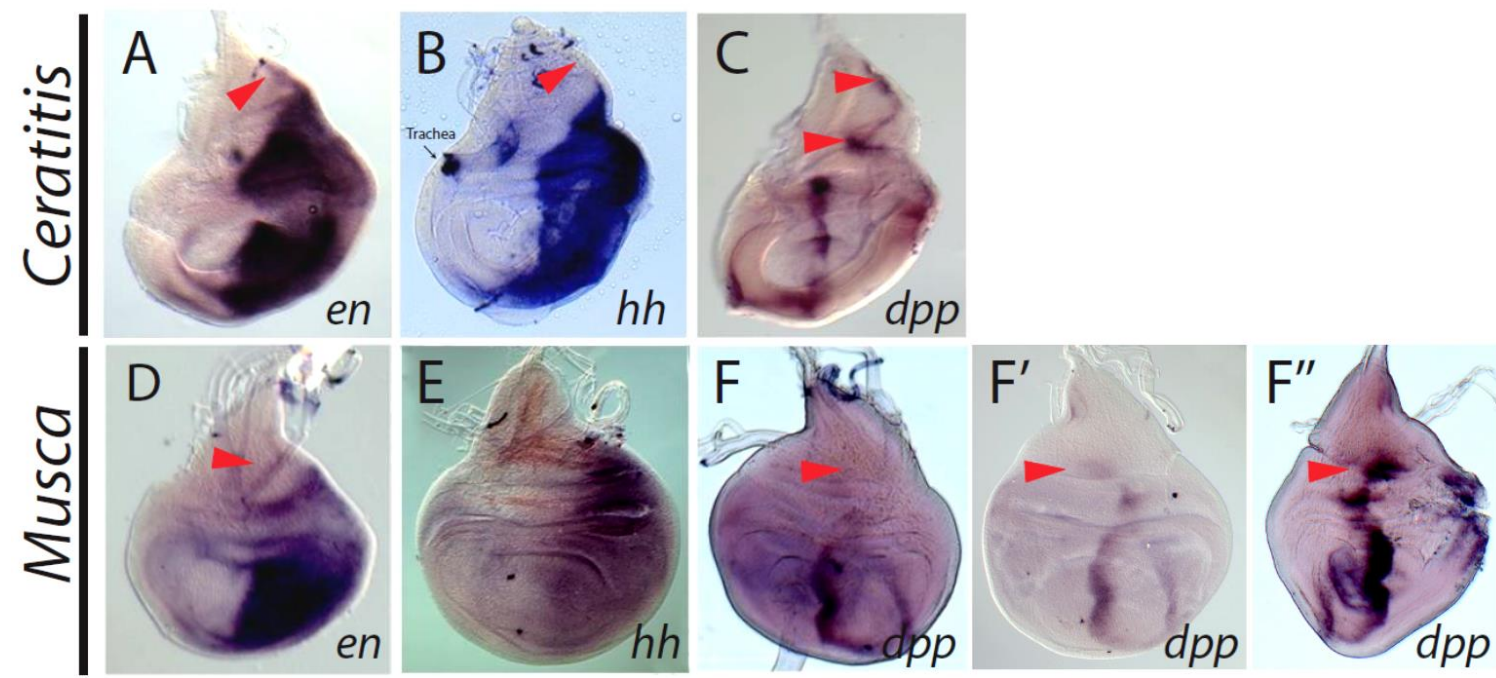

Figure 6.9: Expression of genes involved in the formation of the AP compartment boundary in wing imaginal discs. Expression of $e n$ and $h h$ was present in the posterior compartment in both species. The compartment boundary in Ceratitis discs was strait and precise (A and B). No $h$ h staining was found in the notal region (B, arrowhead). Expression of $d p p$ was detected in a stripe adjacent to the $h h$ producing cells and also in the notum (C, arrowheads). In Musca, en defined a clear border in the wing pouch region, but not in the hinge and notum (D, arrowhead). Staining of $h h$ was very faint with no sharp boundary (E). $d p p$ expression was present in the wing pouch (F); in the wing pouch and hinge region (F'); in the wing pouch, hinge region, and ventral notum (F") possibly depending on the exact age of the dics. No $d p p$ expression was ever detected in the thin strip of tissue going to the disc stalk in Musca (F, F', and F"). In all discs, anterior is to the left and dorsal is to the top. 
Expression of genes establishing the DV boundary in wing imaginal discs was very similar between the analyzed species and looked like in Drosophila. Expression of ap was restricted to cells that give rise to the dorsal structures: the dorsal thoracic body wall structures, notum, scutellum, and dorsal surface of the wing blade (Figure 6.10A and B).

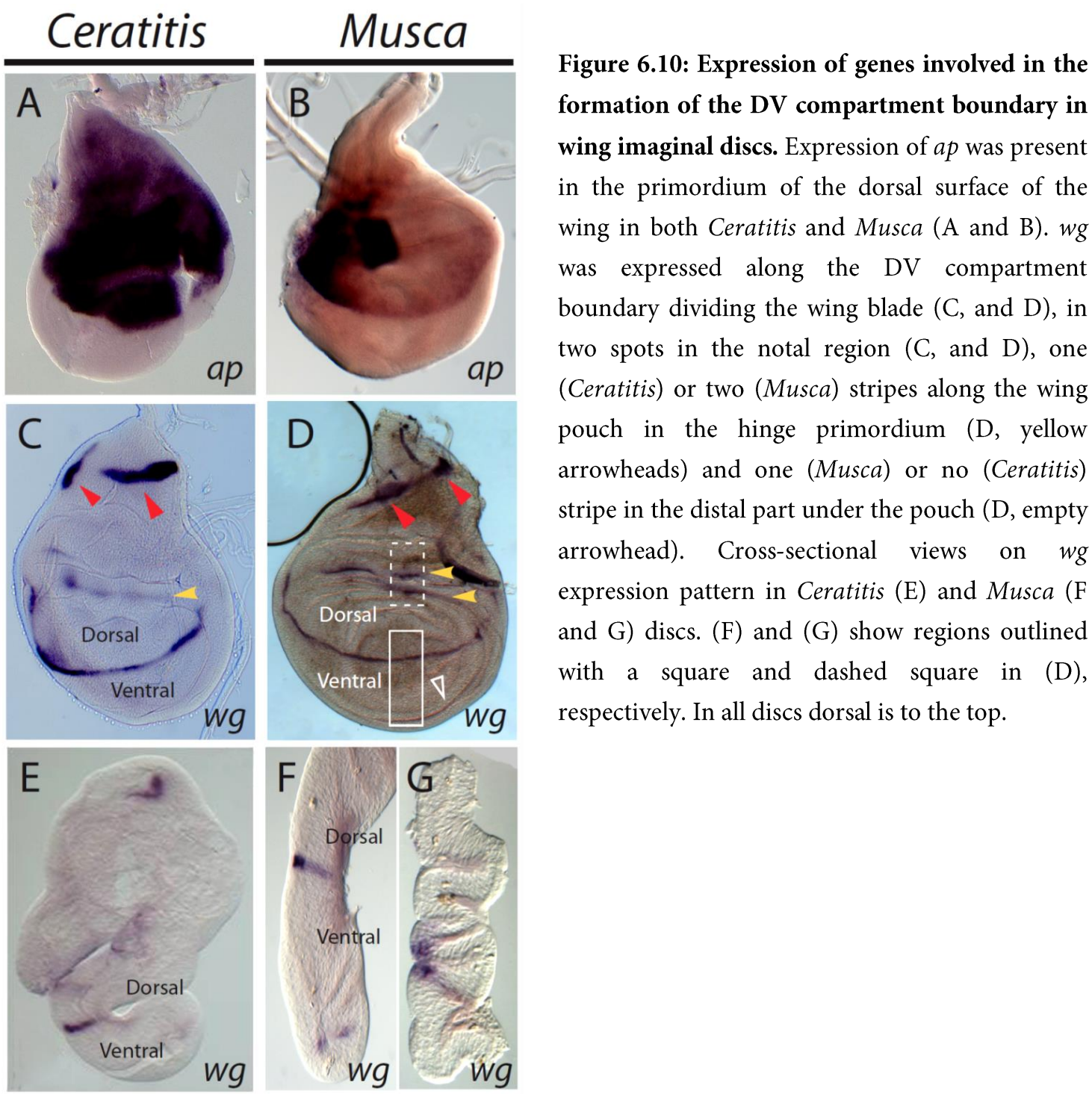

The second analyzed gene required for the DV polarity was $w g$. Its expression pattern highlighted the DV boundary with a thin strip of cells along the presumptive wing margin, where the further dorsal and ventral surfaces of the wing meet (Figure 6.10C and D). In later stages, expression of $w g$ extended and formed two additional stripes along the hinge region of the wing in Musca (Figure 6.10D, yellow arrowheads) and one in the distal part under the pouch (Figure 6.10D, empty arrowhead). In Ceratitis, I only detected one additional stripe in the dorsal part 
framing the presumable wind blade (Figure 6.10C, yellow arrowhead). There were also present two patches in the notal region over the position of the dorsocentral bristle precursors (Figure 6.10B and D, red arrowheads). Because wing imaginal discs in Ceratitis and Musca were heavily folded especially in late larval stages and the staining was not always clear, expression of ap and $w g$ was additionally tested and confirmed in cross-sections of discs (Figure 6.10E, F, and G).

In addition to genes generating the compartment boundaries, I also compared expression patterns of some genes known to be involved in the wing morphogenesis and vein development in Drosophila. First, I tried to obtain expression patterns of salm and kni, which contribute to positioning the longitudinal veins in developing Drosophila wings. In Musca, I was not able to get a specific staining of $k n i$ on wing imaginal discs even though the probe worked well and the staining was specific in embryos (see 6.2.2, Figure 6.15). salm was expressed in the middle of the wing pouch in a round patch with fuzzy borders In Ceratitis, I detected expression of both genes: $k n i$ was expressed in two stripes in the wing blade region, one stripe in the dorsal and one stripe in the ventral hinge, and a small cluster of cells at the margin in the anterior compartment (Figure 6.11A and B); salm expression was found in a relatively narrow strip of cells in the middle of the wing pouch (Figure 6.11C). Expression of both genes was similar to Drosophila expression patterns in the wing pouch. In other tissue of the disc, expression of kni was rather different between Ceratitis and Drosophila.
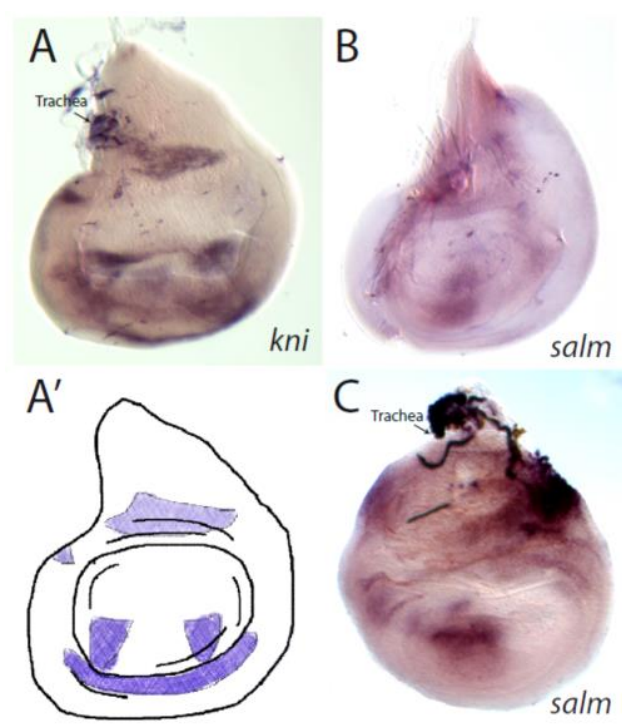

Figure 6.11: Expression patterns of $\mathbf{k n i}$ and spalt in wing imaginal discs. Staining (A) and scheme of kni expression (A') in the Ceratitis wing imaginal disc. Note that the very ventral part of the disc is folded back and it overlaps with the wing pouch resulting into a high background of the staining. Expression of salm (B) was detected in a strip of cells in the middle of the wing pouch in Ceratitis and in a round patch in $\mathrm{Musca}(\mathrm{C})$. 
ISH of all versions of the mad genes did not reveal any specific expression pattern in wing imaginal discs in both species. In contrast, expression of omb was specific in both Ceratitis and Musca. In Ceratitis, I used two 3'-UTR in situ probes: one probe was directed to the full length transcript (new prediction, X1), the second probe targeted both the full length and short version. Expression patterns of the two RNAs seem to be very similar to each other if not identical (Figure 6.12A and B). Expression of both versions of $o m b$ was present in three spatially separated regions. The first region was the very central part of the wing pouch together with the central part of the presumptive hinge (Figure 6.12A and B, outline 1). The second relatively large region was the ventral part of the wing disc including the hinge and pleura primordium (Figure 6.12A and B, outline 2). The smallest and usually faint patch was located at the disc margin in the posterior compartment (Figure 6.12A and B, outline 3). In Musca, I used two in situ probes detecting either previously predicted omb $v .1$ or previously predicted omb v.2. Both predicted genes gave similar expression patterns: a single spot in the middle of the wing pouch (Figure $6.12 \mathrm{C}$ and $\mathrm{D}$, outline 1), indicating that there is only one gene as predicted by my RACE data. Similarly to Drosophila, expression of omb was present in the wing pouch, while in other tissue patterns looked different in the three species.

\section{Ceratitis}

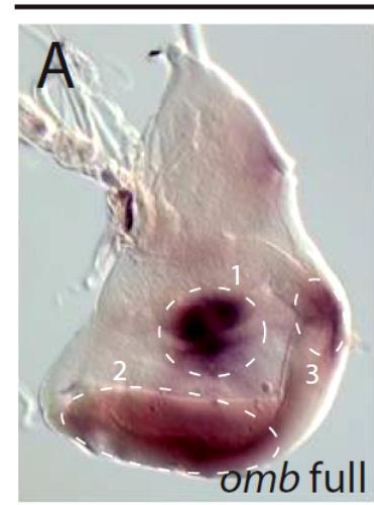

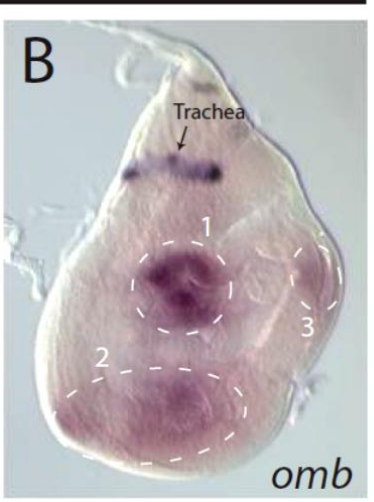

Musca

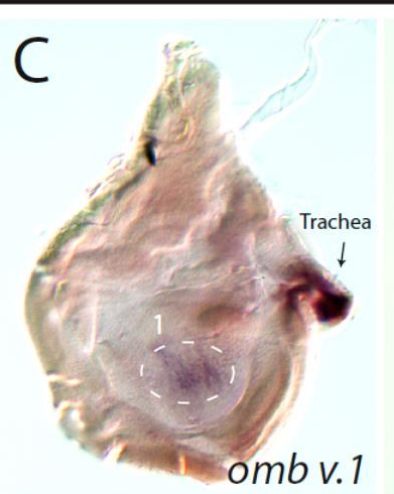

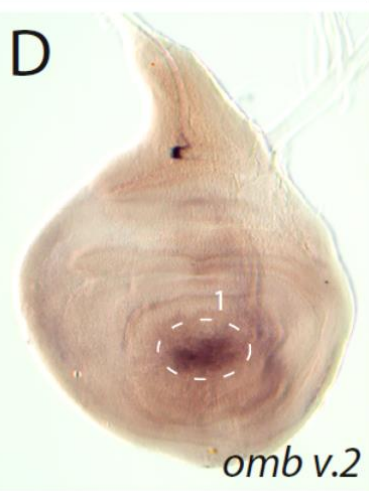

Figure 6.12: Expression patterns of $o m b$ in Ceratitis and Musca wing imaginal discs. Expression of both the long and the short RNA versions was similar in Ceratitis and present in three distinct regions: 1) central part of the wing pouch and hinge; 2) ventral hinge and pleura primordium; 3) small cluster of cells at the posterior margin of the disc. In Musca, expression of predicted omb v.1 and predicted omb v.2 was found in a similar pattern in the middle of the wing pouch (1). In all discs, anterior is to the left and dorsal is to the top. 


\subsubsection{Gene expression patterns in Ceratitis and Musca embryos}

For genes that did not give a specific staining and/or showed an unexpected expression pattern in wing imaginal discs, I additionally performed the whole-mount ISH to visualize location of expressed RNAs in embryos (see Chapter 3: Materials and Methods, Section 3.5.3). Being a segment-polarity gene, en was expressed in isolated bands of cells along the AP axis in both Ceratitis and Musca embryos (Figure 6.13).

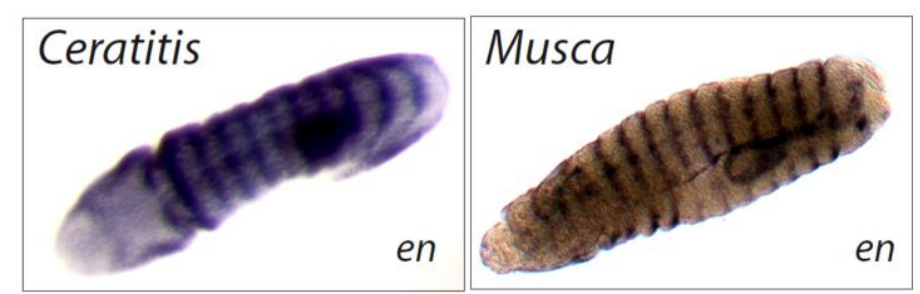

Figure 6.13: Expression of $e n$ in Ceratitis and Musca embryos. Expression of en was found in stripes along the embryo's AP axis. Dorsal view.

Another gene that is involved in the segmentation process during embryonic development in Drosophila is wg. Its expression in the two analysed species showed an expected pattern: narrow stripes of cells located anteriorly to the parasegment boundary (Figure 6.14A and C) as well as some expression in the head region (Figure 6.14A and C, arrows). ap was expressed in the central and peripheral nervous systems (CNS and PNS, respectively) of the developing embryos (Figure 6.14B and D). The early pattern of ap expression in the CNS was similar to the Drosophila pattern: two cells per hemisegment in the thoracic segments and one cell per hemisegment in the abdomen (Figure 6.14B, arrowheads).

\section{Ceratitis}
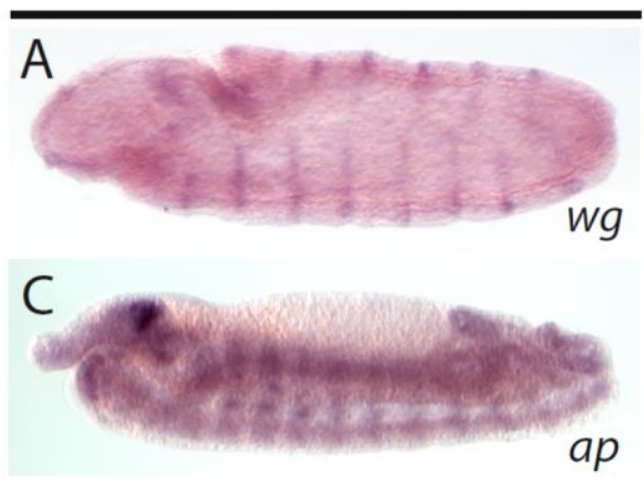

Musca
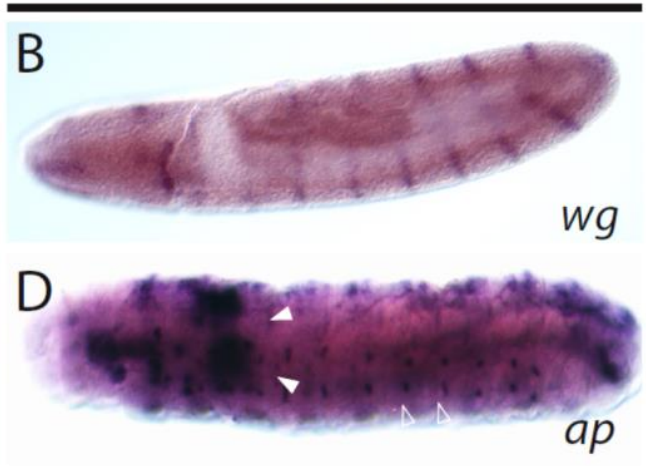

Figure 6.14: Expression of $a p$ and $w g$ in Ceratitis and Musca embryos. Expression of $w g$ in parasegment boundaries and in the head (arrows) in Ceratitis (A), and Musca (C) embryos. The ap transcript expression was present in the CNS and PNS in both Ceratitis (B) and Musca (D) embryos. CNS cells are labelled with empty arrowheads, the brain with solid arrowheads. 
Embryonic expression of kni was also tissue specific. It was detected in every segment and in the head in late Ceratitis and Musca embryos (Figure 6.15A and C). Expression of salm also gave a specific pattern (Figure 6.15B and D).

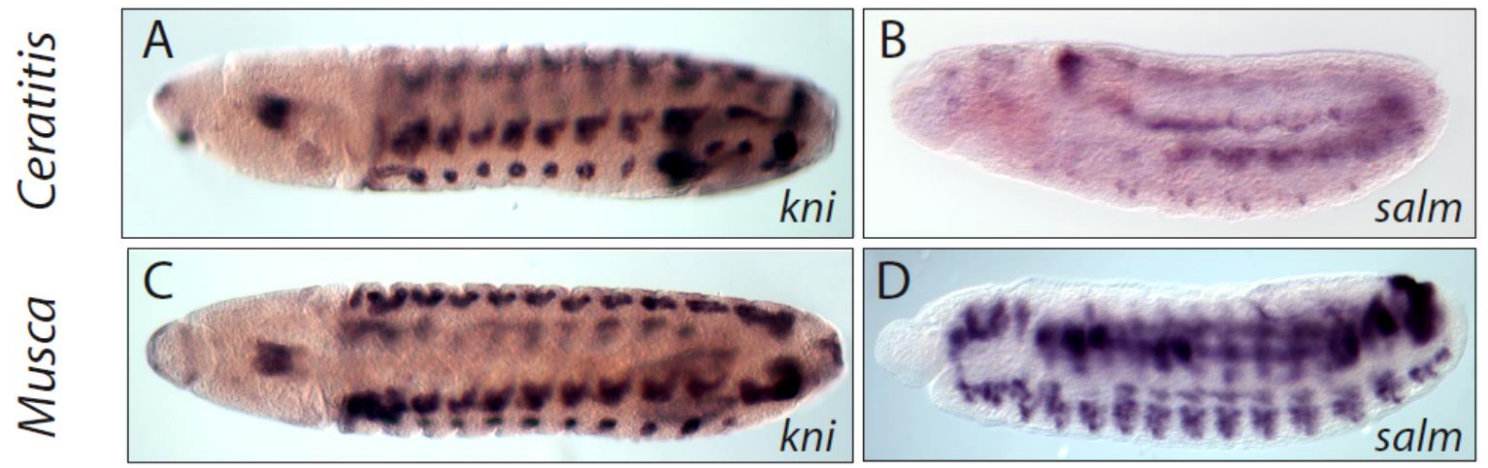

Figure 6.15: Expression of $\mathbf{k n i}$ and salm in Ceratitis and Musca embryos. $k n i$ was expressed in every segment and in the head in Ceratitis (A) and Musca (C) embryos. salm was specifically expressed in Ceratitis and Musca (B and D) embryos.

For the whole-mount ISH of the omb gene, I used the same two probes as for the ISH of wing imaginal discs in Ceratitis. One probe marked the full length transcript (new prediction, X1), the second probe targeted both the full length and short version RNAs. Both probes gave a specific staining in the brain (Figure 6.16). The probe directed to the full length RNA variant gave additional staining of small clusters of cells in every segment in late embryos (Figure 6.16B and C, empty arrowheads).
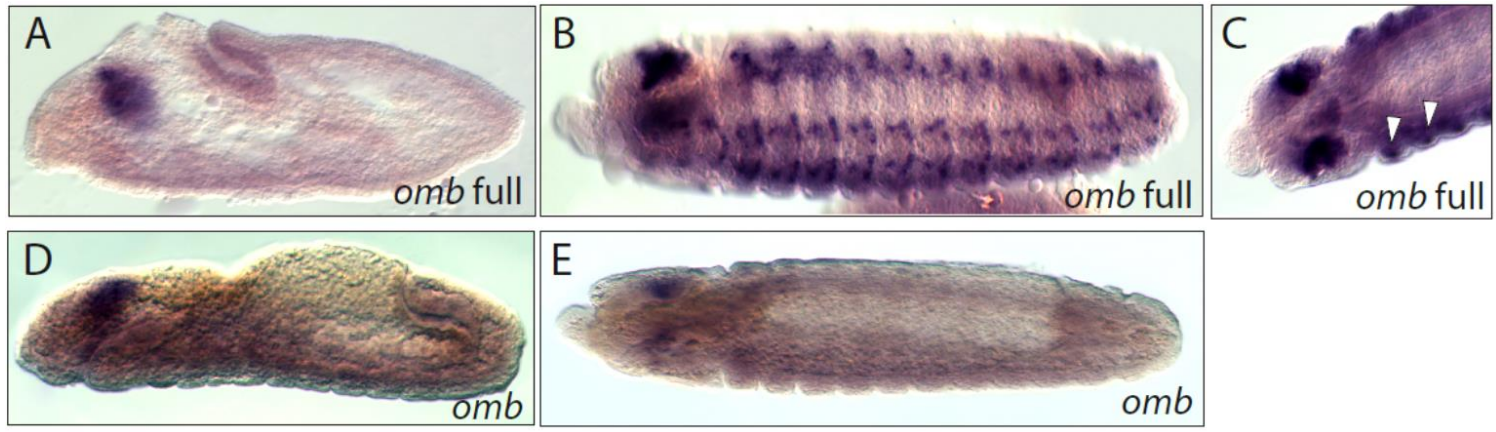

Figure 6.16: Expression of $o m b$ in Ceratitis embryos. Expression of both the full length (A-C) and both RNA variants (D and E) are shown in early (A and D) and late (B, C and E) embryos. Arrowheads mark spots of expression in every embryonic segment in a late stage. Lateral view: (A) and (D); dorsolateral view: (B); dorsal view: (C) and (E). 
In Musca, I also used two in situ probes marking the predicted omb v.1 or predicted omb v.2. Both probes gave similar expression patterns that looked like the full length probe in Ceratitis (Figure 6.17).
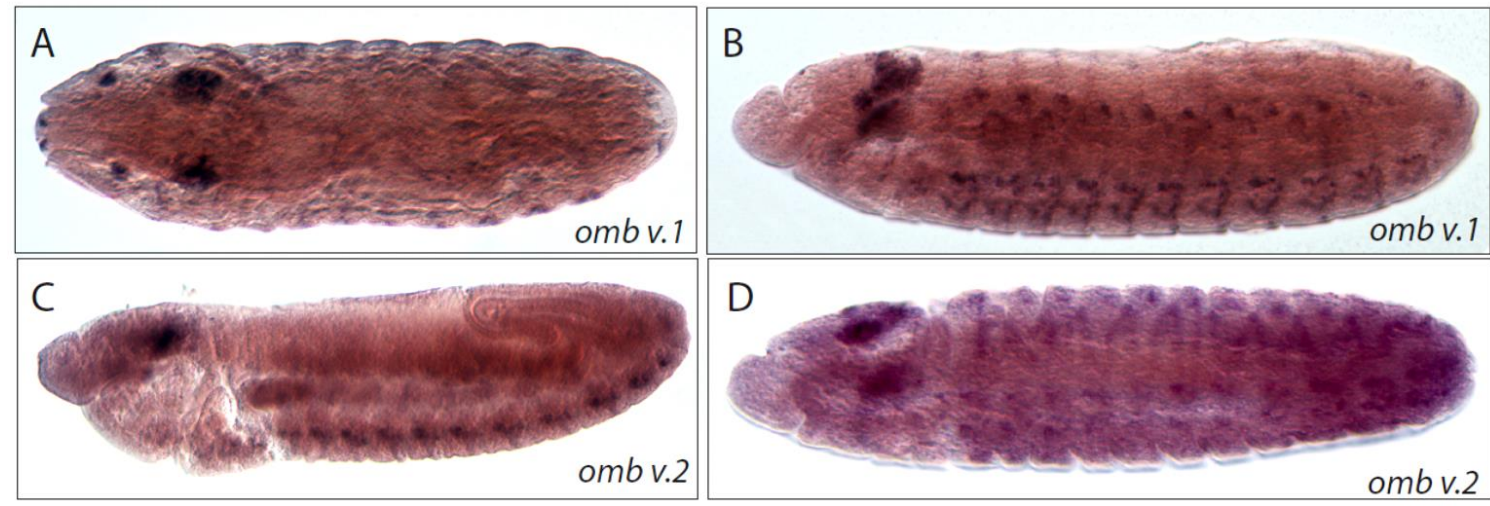

Figure 6.17: Expression of $o m b$ in Musca embryos. Expression of the predicted omb v.1 (A and B) and omb v.2 (C and D) genes in embryos. Dorsal view: (A); lateral view: (B) and (C); dorsolateral view: (D).

Since I did not get a specific staining on wing imaginal discs for any of the mad genes, I tested expression patterns of all of them in Ceratitis and Musca embryos searching for those that may have expression looking like dad in Drosophila embryos. Expression of all mad genes was specific in Ceratitis. Early embryonic stages were highly enriched with mad (Figure 6.18A) and mad-4like (Figure 6.18I) genes. Their expression changed with time but remained until late stages (Figure 6.18D, K, and L). mad-3like was detected later in development being expressed in different metameric structures of the embryo (Figure 6.18F and G). 


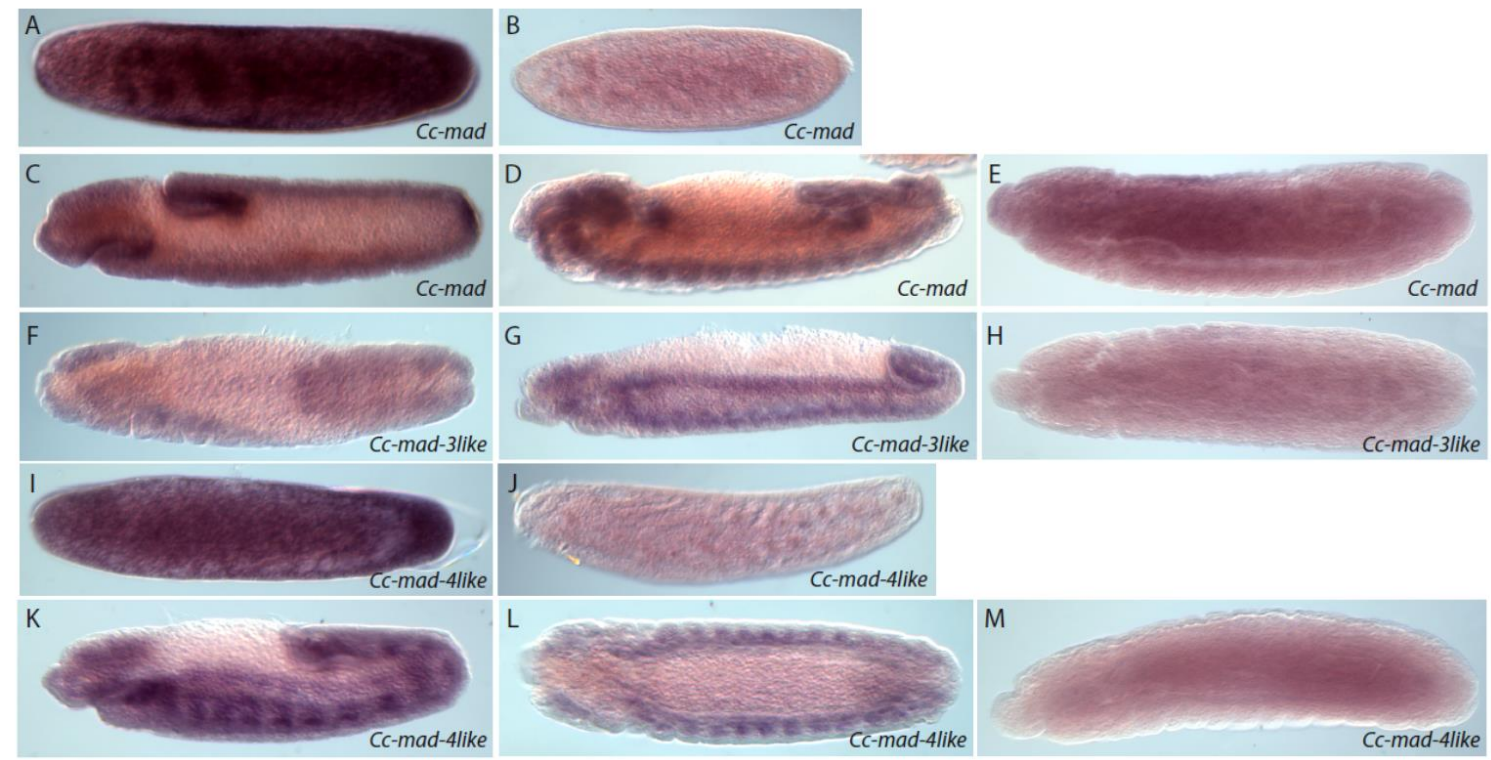

Figure 6.18: Expression of mad genes in Ceratitis embryos of different stages. Expression of the predicted mad (A - E), mad-3like ( $\mathrm{F}-\mathrm{H})$, and mad-4like ( $-\mathrm{M})$ genes was specific. In early embryos, mad and mad-4like were expressed ubiquitously (A and I). Expression of mad, mad-3like, and mad-4like in late embryos was localized in different metameric structures. Negative in-tube control of Musca embryos: B, E, $\mathrm{H}$, J, and $\mathrm{M}$.

Expression of mad-7like, which was the nearest to the Drosophila and Tribolium dad genes on the phylogenetic tree (Figure 6.6), was present since early embryo. The gene was expressed in a stripe along the AP axis and in the posterior region (Figure 6.19A and B). Later, expression was also detected in the mesodermal invagination during gastrulation (Figure 6.C). At late embryonic stages mad-7like was present in a linear series of body segments (Figure 6.19D and E).
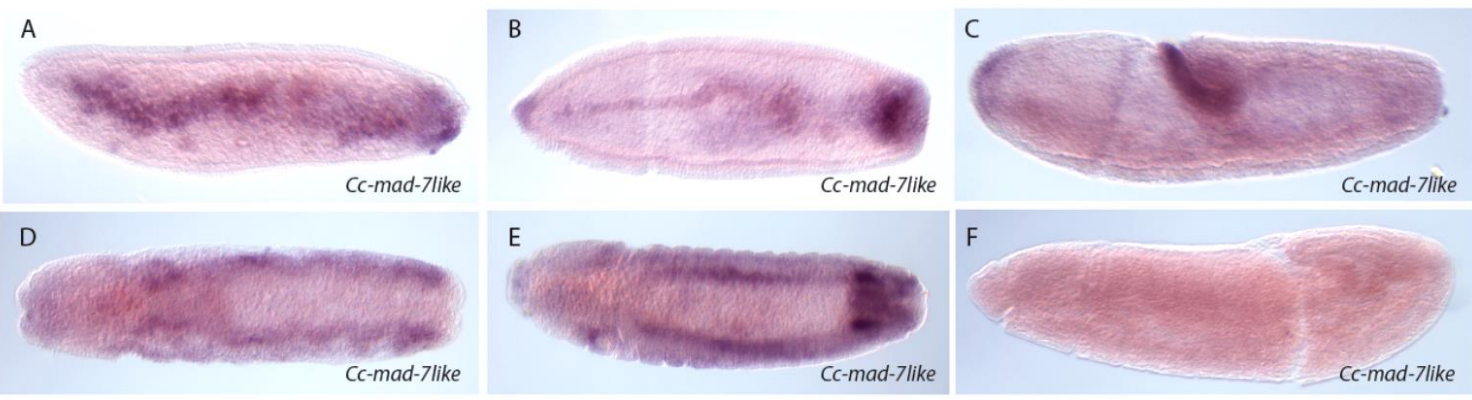

Figure 6.19: Expression of mad-7like in Ceratitis embryos. Expression of the predicted mad-7like in early embryos (A - B), during gastrulation (C), and in late embryos (D and E). Negative in-tube control of Musca embryos: F.

Similarly to Ceratitis, mad and mad-4like were expressed ubiquitously at a very high level in early Musca embryos (Figure 6.20A and J). Expression mad-4like remained ubiquitous in late 
embryos (Figure 6.20L), while mad gained specificity to certain tissues (Figure 6.20C and D). In contrast to Ceratitis, mad-3like was detected neither in early nor in late embryos (Figure 6.20F - I).

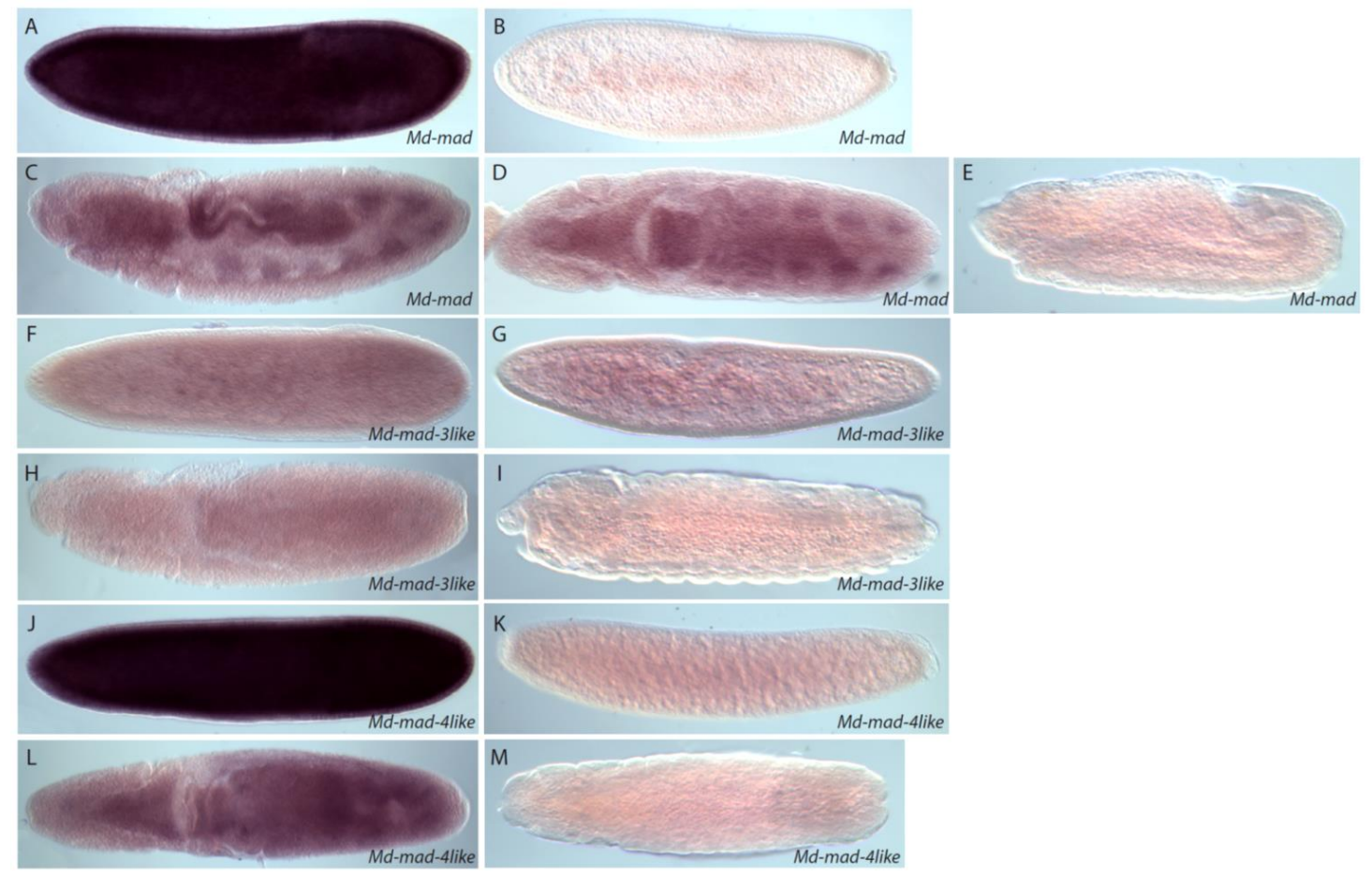

Figure 6.20: Expression of mad genes in Musca embryos of different stages. Expression of the predicted mad $(\mathrm{A}-\mathrm{E})$ and mad-4like $(\mathrm{J}-\mathrm{M})$ genes was specific. In early embryos, mad and mad-4like were expressed ubiquitously (A and J). Expression of mad in late embryos was localized in different metameric structures, while mad-4like retained ubiquitous expression. mad-3like (F - I) was not detected in Musca embryos. Negative in-tube control of Ceratitis embryos: B, E, G, I, K, and M.

Expression of mad-6like in Musca was found in stripes (6.21A) as well as in head and unclear metameric structures later $(6.21 \mathrm{~B}, \mathrm{C}$, and D). At early embryonic stages, there was not specific staining detected.
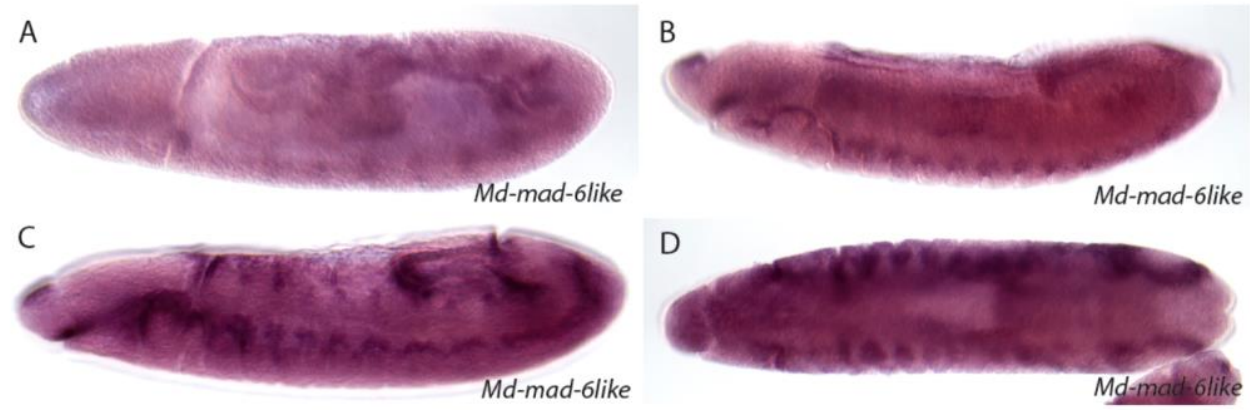

Figure 6.21: Expression of mad-6like in Musca embryos. Expression of the predicted mad-6like gene $(A-D)$ was tissue specific in late embryos. No expression was found at early embryonic stages. 


\subsection{Discussion and future work}

\subsubsection{Drosophila, Ceratitis, and Musca homologous genes}

Comparing protein and gene sequences of the three species, I found a number of homologous genes that are known to be involved in wing development. Homology was first identified by the sequence similarity and then tested with ISH experiments. In case of omb, I found a number of genes that were predicted to be homologous to the Drosophila omb. My RACE-PCR experiments revealed that in Ceratitis there is only one omb gene, which however has two alternative transcripts: the full-length X1 (new annotation) and the short version (omb v.1 old annotation).

After the BLAST search, I got two predicted omb genes (omb v.1 and omb v.2) in Musca. However, this prediction did not find any support with our RACE-PCR. I was not able to identify either 3'-UTR of the predicted omb v.1 or 5'-UTR of the predicted omb v.2. Thus, I assumed that there was only one omb gene in Musca and both predicted RNAs were just parts of a single omb transcript. Further, this assumption was confirmed with ISH experiments (see Section 6.3.4 for details).

For many predicted genes, I also got more than one RNA variant and therefore tested whether they were present in our specific strain. In result, I specified 5'- and 3'-UTRs of the analyzed genes in Ceratitis, identified new non-annotated transcripts for mad and mad-4-like, detected introns in the 5'-UTR of ap and in CDS of mad-7like, and excluded some isoforms from the further analysis because they were not found in our Ceratitis strain. In Musca, I specified 5'- and/or 3'-UTRs of some genes, detected introns in the 5'-UTR of hh and CDS of mad-3like. Thus, all predicted RNA isoforms were found and further analyzed.

With the blast search, I was not able to identify dad in Ceratitis and Musca. All found proteins with a high similarity to Dad or dad were annotated as mad and its protein in these two species. In spite of this discrepancy, I kept all found variants of Mad as possible candidates and included the respective genes in the list for ISH. This decision was based on the three following reasons. Firstly, at the time of my search, the Ceratitis genome had just been released and annotations were partially incorrect (later, this reason found support with omb and salm genes). Secondly, dad did not seem to be conserved among insects. BLASTP of Drosophila Dad always gave the best hit with the respective Mad proteins in many insects (including genera Bombyx, Apis, Fopius and others). Only in Tribolium castaneum, I was able to identify a homolog of 
Drosophila Dad. Alignment of T. castaneum and D. melanogaster Dad proteins showed identity of $36 \%$ only with the query cover $60 \%$. In Ceratitis, Drosophila Dad gave a higher query cover and similar identity on the protein level in comparison with the Tribolium results (PREDICTED: mothers against decapentaplegic homolog 7 isoform X1 (Ceratitis capitata), query cover 99\%, identity 34\%). However, on the gene level, the alignment of Ceratitis mad with Drosophila dad was very bad: only single nucleotides and very short sequences were identical. The third reason to consider predicted Ceratitis mad genes was the fact that Drosophila dad groups together with the predicted Ceratitis mad-7like gene on a phylogenetic tree (Figure 6.6), suggesting high similarity between the genes. Based on this reasoning, I included the mad genes found in Ceratitis and Musca in my further experiments.

\subsubsection{Anteroposterior boundary formation}

When wing imaginal discs are allocated, they are already subdivided into two compartments, the anterior and posterior, with cells that develop differently and form two distinct parts of the adult wing. Cells in the posterior compartment and their descendents express a selector gene en and segregate from the anterior compartment. Analyzing expression of this gene in Ceratitis and Musca wing imaginal discs, I found that patterns differ between the two species. In Ceratitis, staining was present in the posterior compartment in the wing pouch, hinge region, notum, and scutellum (Figure 6.9A). In general, expression pattern of en in Ceratitis looked very similar to the known Drosophila pattern (Kornberg et al. 1985; Brower 1986; Hama et al. 1990). The only difference was that the boundary was less wavy in the notal region in Ceratitis. It was rather a straight line going across the notum to the posterior margin of the disc, while in Drosophila the boundary makes a turn just before the presumptive notal region (Brower 1986). In Musca, expression of en was also detected in the posterior compartment. The boundary was clear in the wing pouch, while in other regions it was fuzzy with no expression in the presumptive scutellum (Figure 6.9D). Importantly, expression of en in embryos was similar between the two studied species (Figure 6.13) and Drosophila (Fisher et al. 2012). However, it is still worth checking invected, which is closely related in sequence to en and also expressed in the posterior compartment of wing imaginal discs in Drosophila (Coleman et al. 1987).

One important role of $e n$ is to label posterior cells and prevent their mixing with cells of the neighbouring anterior compartment (Crick and Lawrence 1975; Morata and Lawrence 1975; 
Lawrence and Morata 1976). Another equally important role is to induce expression of $h$ h in posterior cells exclusively and block ability of these cells to respond to the secreted Hh protein (Tabata et al.1992; Zecca et al. 1995). As a result of this positive regulation, the expression pattern of $h h$ was nearly identical to the expression pattern of en in Drosophila (Lee et al. 1992; Tabata et al.1992). In contrast to these results, expression patterns of these two genes did not coincide completely in Ceratitis and Musca. Expression of $h h$ was restricted to the posterior compartment. However, $h$ h was not detected in the presumable scutellum in Ceratitis even though en was present there (Figure 6.9A and B). In Musca, $h h$ was also absent in this region (Figure 6.9E), suggesting that there could be changes in the gene regulation and that some of $h h$ functions could be governed by other genes.

Activation of $d p p$ is induced by Hh, which is a secreted short-range inducer (Tashiro et al. 1993; Porter et al. 1995) that crosses over the border to the anterior compartment (Lee et al. 1992; Tabata and Kornberg 1994). In Drosophila, $d p p$ is expressed in a narrow stripe of cells at the AP boundary that express neither en nor $h$ h (Masucci et al. 1990). Cells expressing $d p p$ form a discontinuous band along the presumptive proximal-to-distal axis crossing the ventral hinge, the wing pouch, the dorsal hinge; another band is going along the posterior edge of the wing disc, through the notum and the scutellum (Masucci et al. 1990; Blackman et al. 1991). In Ceratitis, expression of dpp was also present in the scutellum region where $h h$ was absent. Otherwise, the pattern was very similar to the one in Drosophila (Figure 6.9C). In Musca, the ability of Hh to activate $d p p$ seems to be spatially and/or temporary restricted. In some cases, the expression pattern of $d p p$ was not complete, even though expression of $h h$ was present in the entire posterior compartment. This means that either some regions did not respond to the Hh signals at all (spatial restriction) or they responded with a delay (temporary restriction).

It is important to mention here that development of wing imaginal discs is different in terms of time among the three species. The Drosophila wing disc develops from a small cluster of 2-20 founder cells that invaginate during embryogenesis and proliferate during the larval and early pupal stages (reviewed in Postlethwait 1978; Cohen 1996). Histological studies in the first instar larvae showed that the wing imaginal discs consist of $\sim 40$ cells each (Madhavan and Schneiderman 1977). Since this time the discs can be identified, dissected and further analyzed. In contrast, imaginal discs do not appear so early in Musca. Some of them may be well-developed in the third larval instar, while others may not appear until the larva reaches its resting period or 
even later (Hewitt 1914). Dissections of larvae at different stages showed that wing imaginal discs could not be found before the late third instar. At this stage, the initially allocated wing primordium starts growing and discs significantly increase in size during several hours. I assume that this rapid growth could possibly explain the described above delay in activation of the $d p p$ expression that was observed in Musca. However, more specific and detailed study should be performed to confirm or to disprove my hypothesis.

In general, however, I found that expression of genes responsible for the formation of the AP boundary was similar in the wing pouch. Borders of expression patterns were clear in most cases and there was no major difference found in comparison to Drosophila. Other tissue, e.g. presumptive hinge and notum, were more species specific and expression of analysed genes was not always similar to the patterns known from Drosophila. For example, absence of the $d p p$ expression in the scutellum region was a specific feature in Musca. In Drosophila, one of the functions maintained by $d p p$ there is positioning of the dorsocentral and scutellar bristles of the notum. Dpp regulates wg expression via plural mechanisms involving pannier and $u$-shaped, so that only cells receiving optimal level of the Dpp signal express wg. (Huang et al. 1991; Sato and Saigo 2000; Tomoyasu et al. 2000). This conserved gene regulatory network results into a constant number of thoracic bristles throughout the Drosophilidae: most species have two dorsocentral and two scutellar bristles (reviewed in Simpson 2007). The number, position and length of the thoracic bristles are characters that are constantly used in determining many species. In Musca, however, this characters vary and especially the number of postsutural dorsocentrals (d'Assis Fonseca 1968). From these gene expression results, I assume that the observed atypical expression of $d p p$ might affect the conserved network in the notal region resulting into this variation.

\subsubsection{Dorsoventral boundary formation}

The DV axis originates much later in development than AP (Garcia-Bellido et al. 1976; Diaz-Benjumea and Cohen 1993; Ng et al. 1996). During the L2 stage, both anterior and posterior compartments of the wing imaginal disc are subdivided once again, and the two genes that assure the correct subdivision are $a p$ and $w g$. In Drosophila, expression of $w g$ in wing imaginal discs can be detected starting from the beginning of the second instar (Couso et al. 1993). This gene has a dynamic expression pattern and plays a key role in positioning ap expression, which can only be detected from the mid second instar (Williams et al. 1993). In my experiments, I used wing 
imaginal discs of the late L3 stage, and this means that both gene should be in the active state during this time.

I found that in both Ceratitis and Musca ap was expressed in the dorsal region similarly to the Drosophila pattern (Cohen et al. 1992; Diaz-Benjumea and Cohen 1993; Williams et al. 1993). In embryos, ap expression was also similar between the three species (Figure 6.10A and B; Fisher et al. 2012). wg expression was very similar in embryos (Figure 6.14A and C; Fisher et al. 2012), while in wing discs I found some variation between the species. First of all, I found two clearly distinct patches in the notal region in Ceratitis and in Musca, while in Drosophila it was shown that there is only one broad band present in the dorsal part of the disc (Couso et al. 1993; Ng et al. 1996). In the two studied species, this double pattern could possibly be involved in the allocation of bristles, which have different positions in Drosophila (d'Assis Fonseca 1968; Wülbeck and Simpson 2000). Changes in expression of $w g$ may directly or indirectly lead to alteration in expression patterns of other transcriptional regulators, such as scute (via sgg/GSK3), asense, pannier and others, that are known to define bristle precursors (Wülbeck and Simpson 2000; Yang et al. 2012). Additionally, I detected only one ring in Ceratitis, while there were two ring-like expression domains in the hinge region in Musca and Drosophila (Couso et al. 1993; Swarup and Verheyen 2012).

Similarly to the results for the AP boundary, I did not find major differences in $w g$ and ap expression in the wing pouch. Expression patterns were rather similar to the one known from Drosophila. The observed differences in the wg expression concerned the presumptive hinge and notum/scutellum regions.

\subsubsection{Wing patterning and vein development}

In Drosophila, salm and $k n i$ are expressed in the wing pouch, where they are required to initiate development of the longitudinal veins (Lunde et al. 1998; Lunde et al. 2003; Crozatier et al. 2004). I checked expression of these two genes in embryos and wing imaginal discs in Musca and

Ceratitis. I found a tissue specific expression of both genes in embryos of the two species, and the expression was similar to the one found in Drosophila (Fisher et al. 2012). These results confirmed that my probes were specific and the ISH protocol worked. Surprisingly, using the same probes, I was not able to identify expression of $k n i$ in wing imaginal discs in Musca. In Ceratitis, expression of kni was specific but the pattern differed from the expected one. In Drosophila, kni marks 
primordium of the $R_{2+3}$ vein with a break at the presumable wing margin (Lunde et al. 1998; Lunde et al. 2003). In overstained discs, $k n i$ can also be detected in a weak stripe in the $\mathrm{CaA}_{1}$ primordium and entire wing pouch at a low level. In Ceratitis discs, I found kni in two stripes in the wing pouch in the approximate position of the both CaAl and $\mathrm{R}_{2+3}$ primordium. Additional expression in the dorsal and ventral hinge restricting the presumable wing blade showed that $k n i$ was expressed not only in the wing pouch. I also tested both prediction of salm in Ceratitis, but a specific staining was only obtained with the new prediction. Overall, expression of both genes, salm and $k n i$, was not very clear and often borders were fuzzy and imprecise. Therefore, it is important to test knirps-related and sal-related, which complement the tested genes and can improve our understanding of the patterns.

Another gene involved in the venation in Drosophila is omb. For this gene, I had two alternative transcripts in Ceratitis, and therefore I tried to distinguish between the two variants and designed two in situ probes. Because the major part of the transcripts was overlapping, it was difficult to design probes that would be exclusively specific for each of RNAs. Thus, I used one probe marking the full length transcript (new prediction, X1), while the second probe targeted both the full length and the short version. Unfortunately, this approach did not reveal significant difference in expression patterns of the two RNAs in our experiments. However, I believe that it would be of great interest to perform more detailed research and possibly a functional analysis to identify roles of the alternative $o m b$ transcripts.

ISH experiments showed that expression patterns of both predictions of omb were identical in Musca (Figure 6.12C and D). This finding supported the hypothesis that similarly to Drosophila there was only one omb gene and one omb transcript in Musca. I also found that the embryonic expression of omb was alike in the three species: it was present in the nervous system and the head epidermis primordium (Figure 6.16 and 6.17, Poeck et al. 1993).

It was previously shown that in Drosophila wing discs, omb is usually expressed in a very broad stripe covering almost the entire wing domain and middle hinge region. There, together with salm and sal-related, omb responses to Dpp in a dose-sensitive manner and defines the more distal veins $\mathrm{R}_{2+3}$ and $\mathrm{CuA}_{1}$ (Nellen et al. 1996; Lecuit and Cohen 1998). The omb expression pattern was only partially similar between the two analyzed species and Drosophila. The common feature that all three species share is presence of omb in the middle of the wing pouch, and in Musca it was the only detected expression. In Ceratitis however, transcripts of the omb gene were 
also found in two other regions: ventral hinge with the pleura primordium and a small cluster of cells at the posterior margin of the disc. The ventral hinge and pleura are stained in Drosophila as well, but in this species the staining is not discrete as it is in Ceratitis. The staining of a small cluster of cells at the posterior margin was also present in some results by other researchers (Nellen et al. 1996; Shen et al. 2008), but it was never mentioned in the description of the omb pattern and its function there is still unknown.

Staining of various mad genes was either unspecific (mad-3like in Musca) or present in different metameric structures (e.g. mad-7like in Ceratitis and mad-6like in Musca). To specify these structures, it is required to perform additional double in situ staining with genes that are tissue and organ specific in Drosophila. Altogether, none of the analyzed mad genes was expressed in the dorsal ectoderm, which is known for dad expression in Drosophila embryos (Marty et al. 2000), supporting the idea that none of the analyzed genes was homologous to $\mathrm{dad}$. 
92 | GENE EXPRESSION IN FLY EMBRYOS AND WING IMAGINAL DISCS 


\section{GENOME EDITING WITH THE CRISPR/CAS9 SYSTEM}

To obtain the nine key parameters governing the Dpp gradient, one would need to experimentally measure only three of them (source width $\omega$, production rate $\vartheta$, and concentration $C(x, t)$ ), while other kinetic parameters can be derived and computed from these three (Chapter 2: General introduction, Section 2.3; Kicheva et al. 2007; Wartlick et al. 2011b). Here, I develop and describe an approach that would allow to measure the first two parameters, $\omega$ and $\vartheta$, in Ceratitis and Musca flies. I have already started practical realization of this approach and this chapter includes some preliminary results of this work.

\subsection{Labelling the Dpp gradient}

To label the Dpp gradient in wing imadinal discs, I aimed to generate transgenic lines of Ceratitis and Musca flies. Each line was supposed to carry two different fluorescent proteins (FP) driven by $d p p$ and dad promoters. In this case, I would obtain flies that were similar to Drosophila used in the research by Wartlick et al. (2011b). To do so, I had to identify promoter regions and minimal enhancers of these genes and $d p p$ in particular. In Drosophila, the 3 ' regulatory region of dpp comprises a sequence that is more than 25kb long (Blackman et al. 1987; St Johnston et al. 1990). This long regulatory region includes the so-called blk promoter, the 800 bp minimal regulatory element that is sufficient to drive expression of dpp in wing imaginal discs (Müller and Basler 2000). I used the blk promoter sequence to blast against Ceratitis and Musca genomes. Unfortunately, this search did not give any result and I, therefore, had to change the strategy.

Knowing that it is not possible to replace any of $d p p$ copies or anyhow disrupt it due to the embryonic lethality (Irish and Gelbart 1987; Wharton et al. 1993), I developed an approach that allows me to mark Dpp without decreasing its functionality. I decided to generate a marker strain that would carry a FP with its own promoter flanked with attP sites downstream the $d_{p p}$ gene (Figure 7.1). Presence of attP sites would allow me to replace the FP with any other construct later via site specific recombination. To direct the attP-FP-attP construct to the specific genomic region, I chose the CRISPR/Cas9 system. It was recently developed (Hale et al. 2012; reviewed in Hsu et al. 2014) and successfully tested in bacteria (Wiedenheft et al. 2012), plants (Jiang et al. 2013), human cells (Cho et al. 2013) and animals, such as Drosophila (Gratz et al. 2013), Tribolium (Gilles et al. 2015), zebrafish (Hisano et al. 2015), and others. 


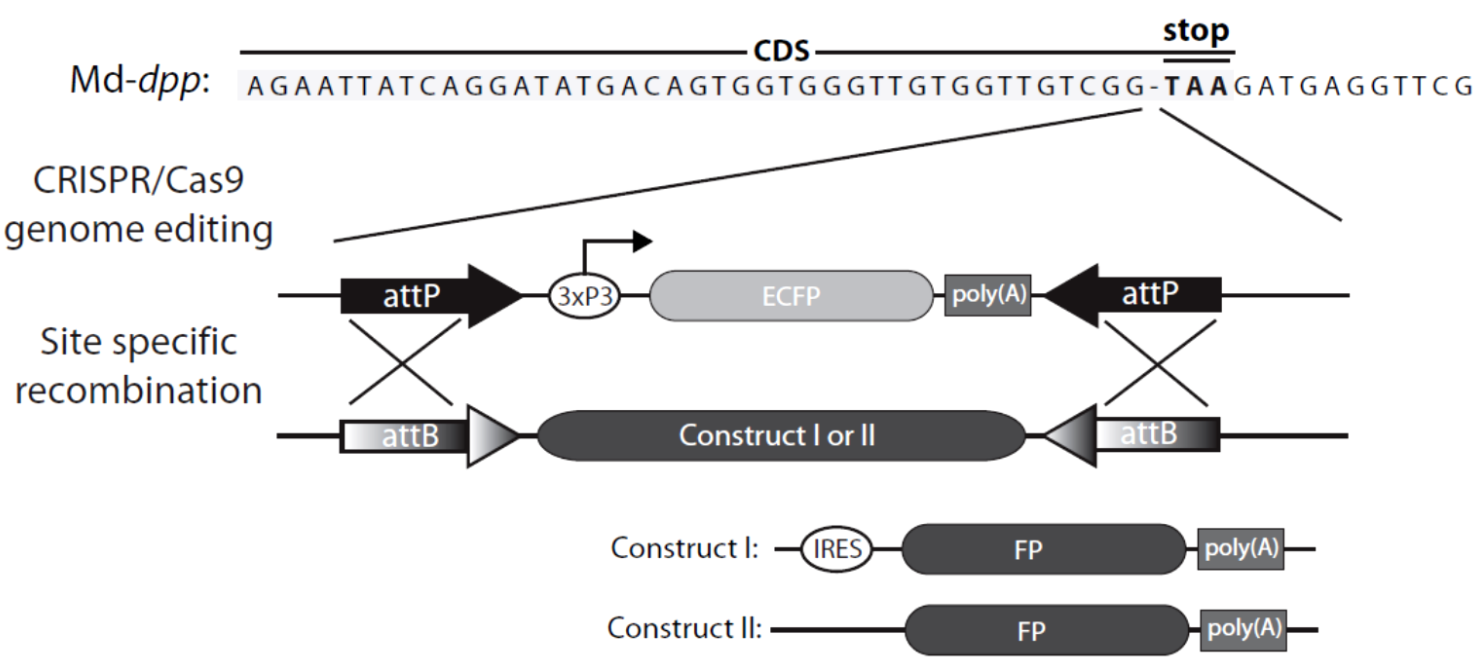

Figure 7.1: Strategy to generate a transgenic Musca strain. Genome editing with the CRISPR/Cas9 system allows generating a marker strain that can further be used for the site specific recombination to mark the Dpp gradient in wing imaginal discs. Md - Musca domestica.

\subsection{Design of constructs for genome editing in Ceratitis and Musca}

\subsubsection{Design of sgRNAs}

Before designing gRNAs targeting dpp in Ceratitis and Musca, I amplified the 3'-UTR of this gene using cDNA of the strains I further plan to use for generating marker strains: C. capitata Egypt II and M. domestica ITA1. The UTRs of both species were sequenced to avoid SNPs that may affect efficiency of gRNAs and/or further recombination with provided vectors. Based on these sequences, I designed six gRNAs: three for Ceratitis and three for Musca integrations (Figure 7.2, Table 7.1). Each gRNA was a 20-bp sequence that targets $d p p$ at the end of the gene. Since I planned to use the CRISPR/Cas system derived from Streptococcus pyogenes, my target DNA sequences precede a 5'-protospacer-adjacent motif (5'-PAM) (Jinek et al. 2012).

Table 7.1: Description and sequences of the designed gRNAs.

\begin{tabular}{|c|c|c|}
\hline Species & \# and orientation & \multicolumn{1}{c|}{ Sequence-PAM } \\
\hline \multirow{3}{*}{ Ceratitis capitata } & $76 \mathrm{~F}$ & GGCTGTGGGTGTCGATAATA-NGG \\
\cline { 2 - 3 } & $77 \mathrm{~F}$ & TGTCGATAATAAGGACATTA-NGG \\
\cline { 2 - 3 } & $78 \mathrm{~F}$ & AAGGACATTACGGCTATTGA-NGG \\
\hline \multirow{3}{*}{ Musca domestica } & $3 \mathrm{~F}$ & CAGTGGTGGGTTGTGGTTGT-NGG \\
\cline { 2 - 3 } & $4 \mathrm{~F}$ & GATATGACAGTGGTGGGTTG-NGG \\
\cline { 2 - 3 } & $5 \mathrm{~F}$ & TATCAGGATATGACAGTGGT-NGG \\
\hline
\end{tabular}




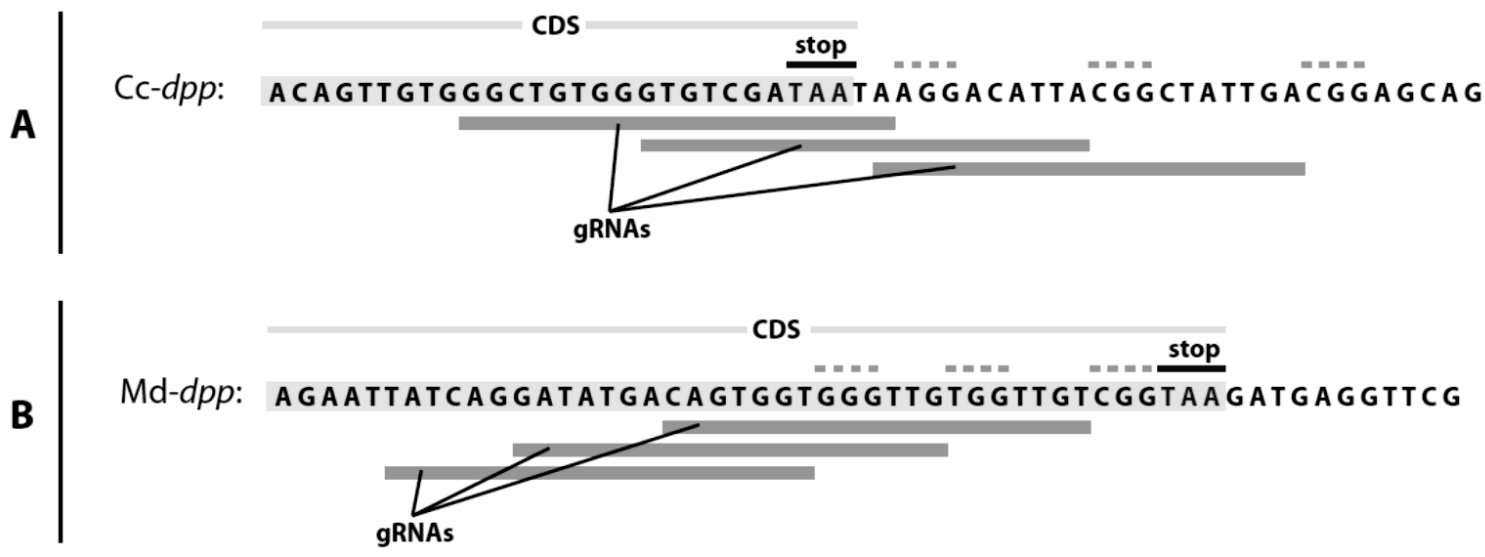

Figure 7.2: Positioning of gRNAs targeting $d p p$ in C. capitata (A) and M. domestica (B). "stop" marks the stop codon, CDS - coding DNA sequence, 5'-PAM is marked with a dashed green line for each gRNA.

All six gRNAs were used in either way to generate sgRNAs (see Chapter 3: Materials and Methods, Section 3.6.1), which were then tested in vitro. Efficiency of sgRNAs was checked by restriction using a fragment of $d p p$ as a template and Cas9 as the restriction enzyme.

\subsubsection{Design of vectors for the homology directed repair}

After the double cut is introduced by RNA-guided Cas9 nuclease, reparation can go in any of these three ways: the non-homologous end joining, microhomology-mediated end joining or homology directed repair (HDR). To carry the HDR, it is required to provide a plasmid-based donor repair template that contains homology arms flanking the site of modification (Smithies et al. 1985; Thomas et al. 1986; Hisano et al. 2015). The homology arms can vary in length, but they are typically longer than $500 \mathrm{bp}$ (Thomas et al. 1986; Hasty et al. 1991). For this study, I decided to use $\sim 1 \mathrm{~kb}$ homology arms to increase efficiency and specificity of the repair. The homology arms for the Musca vector were amplified from gDNA and one arm was cloned (Appendix 11.5, pSLfa1180fa-Md:HAL-3xP3-ECFP). In result of this cloning, I have got 39bp between the homologous arm and first attP repeat left from the backbone, two of which were deleted by a mutagenesis PCR to place the whole construct in the required frame. The other homology arm can be cloned into the EcoRV restriction site downstream the second attP site. As a reporter gene, I use ECFP in this construct. Preparing the vector for HDR in Ceratitis, I have successfully cloned the construct ECFP-poly(A)-attP (Appendix 11.5, vector pSLfa1180fa-Cc:ECFP), while the promoter (ie1) and enhancer (HR5) as well as homology arms remain to be cloned. 


\subsection{Discussion and future work}

\subsubsection{Efficiency of the designed sgRNAs}

Quality of sgRNAs is a crucial factor that may significantly affect the CRISPR/Cas9 genome editing system. I tested six designed gRNAs in vitro, and they effectively worked in these conditions. However, in vivo genome engineering is more complex and usually less efficient (Hwang et al. 2013; Mali et al. 2013; Gratz et al. 2014). For this reason, there are available a lot of guides and critical information on how to design and optimize gRNAs (Doench et al. 2016; Port and Bullock 2016). Even now, gRNAs that were designed according to the theoretical rules with good nucleotide compositions and secondary structures may not work in in vivo experimental systems (Liang et al. 2016). Due to this fact, I designed three different gRNAs targeting a similar region in each species. As an additional factor, I designed our gRNA variants targeting $d p p$ both before the stop codon and after it. I included these two alternatives because the target sequence can be modified (rebuilt or degraded) during HDR to recapitulate the sequence provided with the vector. However, before using this gRNAs, I strongly suggest testing them with a denaturing agarose gel electrophoresis and chose the most stable variant.

\subsubsection{Generation of the marker strain}

To generate Ceratitis and Musca marker strains, I planned to integrate a construct carrying an FP gene before the stop codon of $d p p$. For this, I designed vectors for HDR that include the following construct: attP in the forward orientation, promoter sequence, ECFP, and attP in the inversed orientation. As promoter sequences, I chose 3xP3 for Musca flies and HR5-ie1 for Ceratitis because they were previously shown to work in these species (Hediger et al. 2004; Gong et al. 2005). Introduction of the described above construct would replace the original stop codon of $d p p$ with a stop codon located in the first attP repeat (Figure 7.3). This would result into a slightly modified Dpp with extra 28 AA in C-terminus. This tag should not affect the functionality of the protein because there are already known similar modification and even functional chimeric protein Dpp-GFP (Teleman and Cohen 2000; Kicheva et al. 2007; Wartlick et al. 2011b). 


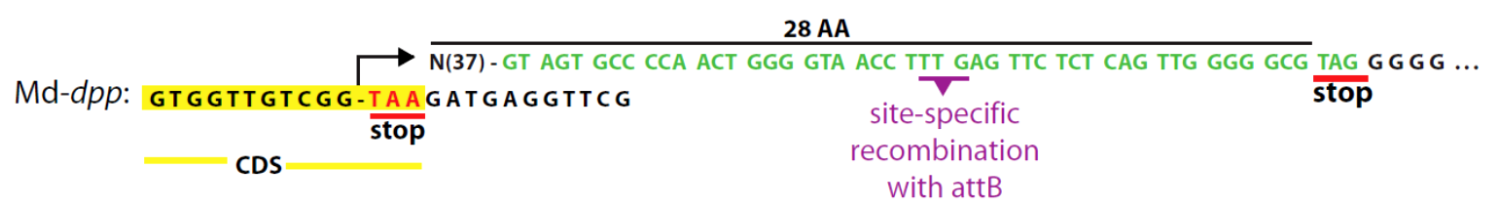

Figure 7.3: Example of integration in the Musca dpp gene. Red letters mark the original stop codon of $d p p$, CDS - coding DNA sequence, attP site is shown with green letters. The tag of the modified $d p p$ is $84 \mathrm{bp}$, which correspond to $28 \mathrm{AA}$.

The positive integration of the construct in the fly genome could possibly be detected during the embryonic and larval development when $d p p$ is active and maintains its functions. Another possibility is to screen for adult Musca flies with blue eyes since $3 \mathrm{xP} 3$ is a tissue specific promoter that is active in ommatidia and ocelli (Horn and Wimmer 2000; Sheng et al. 1997). In this respect, I propose to use two different Musca strains: the Italian strain ITA-1 and white-eyed strain M3-6. ITA-1 is a wild type strain. These flies have normal wings but they also have normally pigmented eyes. Thus, red eyes of adults make it difficult to screen for blue fluorescence. To overcome this disadvantage, I propose to check flies during late pupal stages, when the animal is already formed but the eye pigment is not present yet. As an alternative, it is also possible to use white-eyed mutant flies of the M3-6 strain. However, these flies have another specific feature: pointed wings in females. In this case, screen should be done for positive males, which are possible to cross with ITA-1 females. In result, it would be possible to generate a Musca strain with blue fluorescence in eyes and normal wings.

\subsubsection{Further use of the marker strain}

Marker strains generated in the described above way could be further used to mark the Dpp producing cells and the protein itself as well as for other biological experiments. The attP sites of the integrated construct allow replacement of ECFP and the respective promoter with any kind of construct by using site specific recombination and attB sites for it.

To measure the source width $\omega$, one would need to mark cells of wing imaginal discs producing Dpp by replacing 3xP3/(HR5-ie1)-ECFP with IRES-FP, where IRES is an internal ribosome entry site (reviewed in Stoneley and Willis 2004). Different IRES elements were previously shown to work in insect and mammal cells (Carter et al. 2008). The relative activity of the elements differs in diverse insect cell lines, but certain IRES elements showed a good efficiency in Drosophila cells. I assume that they may also work in other closely related species, such as 
Ceratitis and Musca. As an alternative, one may also use regulatory elements of Drosophila heat shock protein $70 \mathrm{mRNA}$, since it is also able to conduct IRES-mediated translation (Hernández et al. 2004). During the site specific recombination, attP and $a t t B$ sites will give rise to the attR site and the stop codon of the original attP will be gone (Figure 7.4A). In this case, a polycistronic mRNA with two ORFs will be transcribed from the active blk promoter. The first ORF will be Dpp and the second ORF will be FP with its own element for translation initiation. Thus, all Dpp producing cells will also produce the integrated FP.

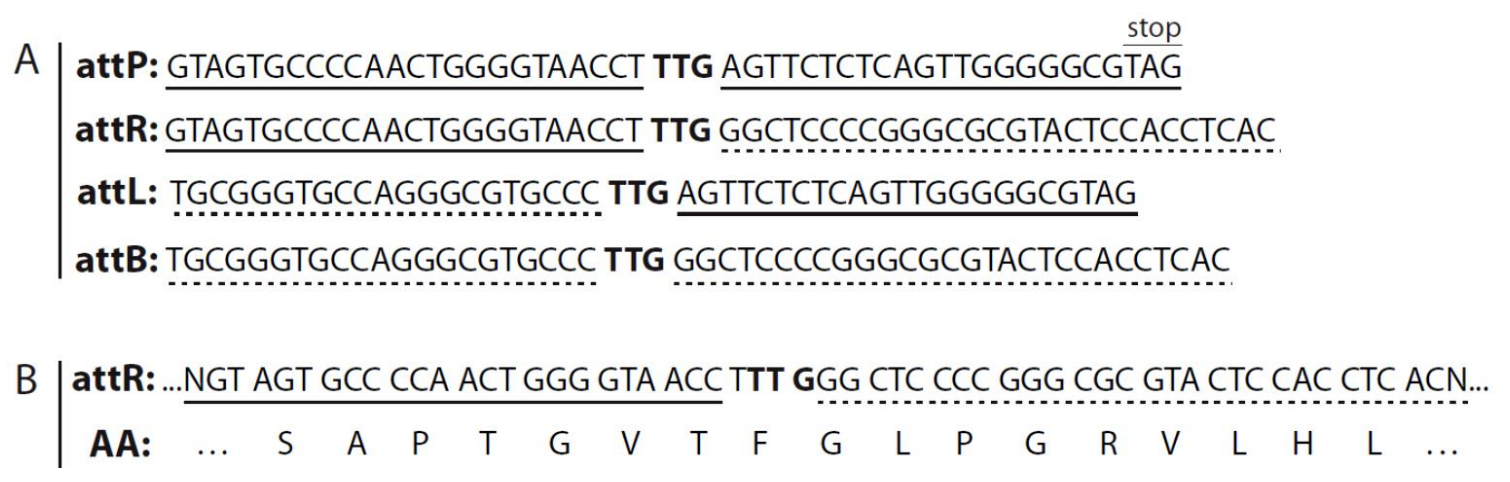

Figure 7.4: DNA sequence of att sites. After recombination, attP and attB sites give rise to two hybrids sites: attL and attR (A). The stop codon present in attP is gone after recombination in attL (B).

The second kinetic parameter of the Dpp gradient that can be measured with the marker strain is the production rate $\vartheta$. By the same site specific recombination, I suggest to replace 3xP3/(HR5-ie1)-ECFP with another FP, e.g. GFP or DsRed. Origin of attL will remove the stop codon and result into the FP fused with Dpp via a linker of 31AA (Figure 7.3 and 7.4B). Consequently, the production rate of Dpp will be proportional (but not equal due to presence of the second $d p p$ copy) to the production rate of the FP. Crossing the two mentioned above strains would make it possible to generate flies with two modified but still functional copies of $d p p$. One copy of $d p p$ would result into the fusion Dpp-FP, while the other will give rise to a polycistronic mRNA encoding another FR. Thus, wing imaginal discs of these flies could be used to measure both $\omega$ and $\vartheta$. To measure the third kinetic parameter $C(x, t)$, another gene modification is required (see Chapter 8: General discussion). 


\section{GENERAL DISCUSSION}

\subsection{Variation of size and wing shape among species}

Body size is one of the key parameters of an animal that has a high impact on different aspects of its life. For our study, I chose three differently sized dipteran species: D. melanogaster, C. capitata, and M. domestica. I analyzed the overall size of these species as well as size of different traits and showed that use of a single body part to estimate the entire body size is not optimal because the value of adult traits may differ among species (Chapter 4: Body size as an integral feature, Section 4.2.6 and 4.3.5). However, it is important to find an impartial and universal approach to monitor activities of natural populations of different species. For this, I propose to use other parameters that better approximate the body size. It can either be environmentally independent pupal traits, such as volume or dry weight, or a BSC computed from measurements of several traits. I applied this approach in our study and used a BSC to estimate the relative wing size in the three species (Chapter 4: Body size as an integral feature, Section 4.2.7 and 4.3.6). I found that the relative WA does not increase with the absolute body size. Among the three flies, Ceratitis was the one that had the largest relative WA, while wings of Drosophila and Musca were similar in size (Figure 4.5). This specificity should be taken into account when analyzing and interpreting features of the wing growth regulation, e.g. Dpp gradient parameters. It is important to test whether values of the parameters correspond to the relative wing size distribution. Additionally, it would be of great interest to check whether the percentage by which concentration of Dpp increases during one cell cycle and motivates cells to divide $(\alpha)$ is similar for the three flies. Analyzing the relative wing size, I found a large dispersion of values in Musca. I think that this specific feature can also be tested and possibly explained by the variation of the percentage $\alpha$ in this species.

Another factor that should be considered during estimation of the Dpp gradient is the sex of the species. In this study, I confirmed a clear SSD in Drosophila and showed that SSD is less uniform in the other two species (Chapter 4: Body size as an integral feature, Section 4.2.2 and 4.3.2). I also showed that response to environmental cues differ between sexes (Chapter 4: Body size as an integral feature, Section 4.2.4 and 4.3.2). This implies a number of growth regulation parameters being sex specific. In this case, the Dpp gradient should be carefully treated in both sexes to clarify whether it is alone responsible for the observed differences or other genes and their pathways contribute to the sex specific response. 
Being a morphogen, Dpp mediates tissue specification and pattern formation in wing imaginal discs (reviewed in Affolter and Basler 2007; Restrepo et al. 2014). Directly or indirectly, Dpp regulates expression of many genes involved in the vein formation and final shaping of the wing. Thus, it would be interesting to test whether parameters of the Dpp gradient are quantitatively similar between similar wing shapes. In this project, I applied geometric morphometrics and estimated similarity of the wing shape between Drosophila, Ceratitis, and Musca in units of Procrustes distance (Chapter 5: Variation of wing shape in different fly species, Section 5.2.1). I found that Ceratitis wings are closer to the shape of Musca wings (Procrustes distance is $0.1857, \mathrm{p}<0.0001$ ) than to Drosophila (Procrustes distance is $0.2357, \mathrm{p}<0.0001$ ). Consequently, one should also expect more similarities in the Dpp gradient of Ceratitis and Musca. In contrast, more differences could, perhaps, be found between Ceratitis and Drosophila because they have the less similar wing shape (Procrustes distance is $0.3072, \mathrm{p}<0.0001$ ).

Analyzing the total SShD and its non-allometric component, I once again showed that the reaction varies between sexes (Chapter 5: Variation of wing shape in different fly species, Section 5.3.3). I found that wing shape of males and females changes differently in different rearing conditions and this is an important point to pay attention to when analyzing the patterning function of the Dpp gradient and searching for pathways that may contribute to the sex specific response in these species.

\subsection{Robustness of the wing development}

Metamorphosis and presence of imaginal discs that later give rise to adult structures implies conservation in development. However, my experiments and observations during dissections of wing imaginal discs revealed some variation in the wing development among the three chosen species. The first difference was temporal: I found that rapid growth of wing imaginal discs occur at different times in these species. Drosophila wing discs are defined during embryogenesis. Most likely, this happens at the same developmental stage in Ceratitis and Musca. Initially, each wing imaginal disc consists of a small cluster of cells that divide and later develop mature discs of approximately 50000 cells (reviewed in Cohen 1996). In Drosophila, this process starts in the first instar larvae and lasts during the whole larval life (Madhavan and Schneiderman 1977). Thus, wing imaginal discs of certain size can be dissected at each larval stage. In contrast, Ceratitis wing discs stay rather invisibly small during the first and beginning of the second instar. They could be 
clearly detected and dissected at the late second instar stage. In Musca, I were not able identify the discs until the late third instar. This observation is in agreement with a previous finding that imaginal discs do not appear and develop at earlier stages in Musca (Hewitt 1914). Thus, wing discs stay small until the late L3 stage and then they start growing quickly and significantly increasing in size in the next hours. This rapid growth and differentiation makes it difficult to precisely estimate the stage of the disc when dissected. Now, this specific feature of Musca development makes it unsuitable for research on the temporal component of the Dpp gradient and its function at different time points (see Chapter 2: General introduction, Section 2.3; Akiyama and Gibson 2015). However, if staging of wing imaginal discs is ascertained, this species will be an interesting model to test whether importance of the morphogen decreases in later stages in a similar way to Drosophila.

I assume that the rapid growth of wing imaginal discs during the late L3 larval stage could also affect expression of some genes involved in the AP boundary formation. I found that $d p p$ expression in wing discs might be temporarily restricted resulting in the observed variation of the expression pattern (see Chapter 6: Gene expression in fly embryos and wing imaginal discs, Section 6.3.1). I always detected $d p p$ transcripts in the wing pouch region of the disc. This might indicate the primary role and high importance of $d p p$ there. In some discs, $d p p$ was expanded along the AP axis towards the hinge region and sometimes ventral notum. I suppose that this expansion of the expression happens during development and the rapid growth of the disc. Thus, discs with a longer stripe of $d p p$ expression might be older. However, this hypothesis should be further elaborated when staging of Musca discs is precisely determined. In any case, observation of different $d p p$ expression patterns suggested that there was a developmental separation of the wing pouch from the other wing disc tissue. Analysis of other wing developing genes further supported this assumption. I found that expression patterns were rather similar among species in the wing pouch region. Main differences in expression were only observed in the presumptive hinge and notum/scutellum regions (e.g. wg and kni) (see Chapter 6: Gene expression in fly embryos and wing imaginal discs, Section 6.3.1).

An additional support to this developmental separation could also be found in the sexual specificity of the response to rearing conditions. Analyzing size changes in response to different rearing conditions, I found a sex-dependent response in wings, which develop from the wing pouch. In contrast, thorax, which is mainly formed by tissue of the notal region of the disc, could 
change differently between sexes (Ceratitis) or similarly (Drosophila) (Table 4.2). In Chapter 4: Body size as an integral feature (Section 4.3.3), I hypothesized how thoraces and wings, which develop from the same larval disc, could differently react to the environment and how thorax could have its temperature sensitivity lost.

Altogether, my data lead to two main conclusions. Firstly, development of the wing imaginal disc has a complex regulation that may differ in regions with different fate. Presence of 'fate-specific' regulation leads to the developmental separation of the wing pouch at some point. This conclusion is also supported by the finding that one Dpp spreading model could not entirely explain size of the wing disc in Drosophila (Harmansa et al. 2015). If we accept that the wing pouch is a developmentally separate unit, we may conclude that the three chosen species are rather similar in the wing development. From this, it follows that the growth model by GonzálezGaitán's group (Wartlick et al. 2011b) could still be applicable to the wing pouch.

\subsection{Amplitude of Dpp gradient in Ceratitis and Musca}

In order to test any Dpp growth regulation model in Ceratitis and/or Musca, one would need to mark the Dpp gradient in this species. Since it was not possible to identify a homolog of the blk promoter in these species (Chapter 7: Genome editing with the CRISPR/Cas9 system, Section 7.1), I designed a novel approach that allows us to generate a marker strain that can be used for different biological purposes.

One of the possible outcomes is a possibility to mark Dpp with a FP and to measure a number of the Dp gradient parameters (Chapter 7 Genome editing with the CRISPR/Cas9 system, Section 7.3). Using the approach I propose, it is possible to mark the Dpp source width $(\omega)$ and production rate $(\vartheta)$. However, to compute other parameters of the Dpp gradient, one would need to detect spreading of the morphogen. Following the Drosophila model, I initially planned to use the upstream region of dad to drive a FP. It fluorescence would depend then on the Dpp signaling and the intensity of fluorescence would be proportional to the Dpp concentration: $\operatorname{IF}[\mathrm{dad}-$ FP] $\sim C_{\text {Dpp }}(\mathrm{x}, \mathrm{t})$ (Wartlick et al. 2011b). Presence of the $C(x, t)$ parameter makes it possible to compute the gradient amplitude $\left(C_{0}\right)$ and the decay length $(\lambda)$ by using an exponential fit function (Figure 8.1). 


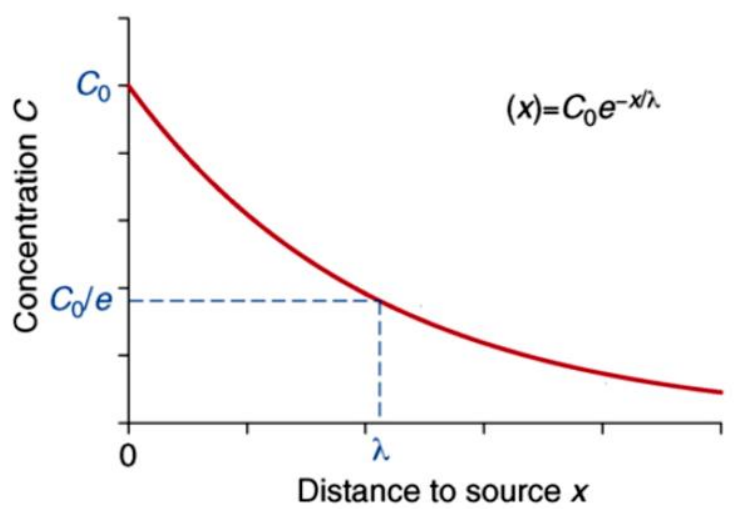

Figure 8.1: Positional information and the Dpp gradient shape. Morphogen concentration as a function of the distance to the source $x$. An exponential gradient is described by the given equation. The gradient amplitude $\mathrm{C}_{0}$ and the decay length $\lambda$ describe the gradient shape. (Kicheva and González-Gaitán 2008)

The two parameters $C_{0}$ and $\lambda$ regulate the Dpp gradient range. Increase in either of the two parameters will lead to a longer distance at which a particular concentration occurs (Figure 8.2).

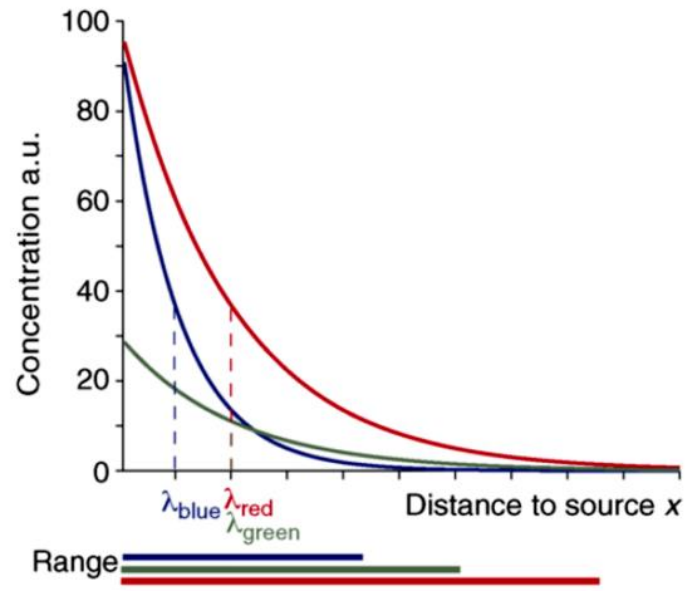

Figure 8.2: The range of the Dpp gradient. The position where detection threshold is reached is affected by both $C_{0}$ and $\lambda$. The blue and red gradients have the same $C_{0}$, but different $\lambda$ (dotted line), while the green and the red have different $C_{0}$, but the same $\lambda$ (and therefore the same diffusion and degradation properties of the target). The three gradients have different ranges although they share either $C_{0}$ or $\lambda$. (Kicheva and González-Gaitán 2008)

Knowing the Dpp concentration and its temporal changes, I could calculate the kinetic parameters of the gradient: degradation rate $\left(k, k=\frac{\vartheta}{2 C_{0}}\left(1-e^{-\frac{\omega}{\lambda}}\right)\right)$, diffusion coefficient $(D$, $\left.D=\lambda^{2} k\right)$ as well as the percentage by which $C_{D p p}$ increases during one cell cycle $(\alpha): \alpha=\frac{\Delta C_{\text {cell }}}{C_{\text {cell }}}$.

Values of these gradient properties quantitatively describe it and allow comparison between the species. The weakest link in this method is that I was not able to identify the dad gene in Ceratitis and Musca with either BLAST search or ISH experiments (Chapter 6: Gene expression in fly embryos and wing imaginal discs). I also noticed that many other insects lack dad homologs. If it also holds true for the chosen species, I propose to slightly modify the approach and use another Dpp target gene that response in the concentration dependant manner (Hadar et al. 2012). 
104 | GENERAL DISCUSSION 


\section{REFERENCES}

Affolter M, Basler K (2007) The Decapentaplegic morphogen gradient: from pattern formation to growth regulation. Nat Rev Genet 8: 663-674. Doi: 10.1038/nrg2166

Akiyama T, Gibson MC (2015) Decapentaplegic and growth control in the developing Drosophila wing. Nature 527: 375-378. Doi: 10.1038/nature15730

Allen CE, Zwaan BJ, Brakefield PM (2011) Evolution of sexual dimorphism in the Lepidoptera. Annu Rev Entomol 56: 445-64. Doi: 10.1146/annurev-ento-120709-144828

Alves SM, Bélo M (2002) Morphometric variation in the housefly, Musca domestica (L.) with latitude. Genetica 115: 243-251

Anderson WW (1966) Genetic divergence in M. Vetukhiv's experimental populations of Drosophila pseudoobscura. 3. Divergence in body size. Genet Res 7: 255-266

Angerer LM, Angerer RC (1992). In situ hybridization to cellular RNAm with radiolabeled RNA probes. In: Wilkinson DG, editor. In situ hybridization: a practical approach, the practical approach series. Oxford, UK: Oxford University Press pp. 15-32

Atkinson D (1994) Temperature and organism size - a biological law for ectotherms? Adv Ecol Res 3: 1-58

Badyaev AV (2002) Growing apart: an ontogenetic perspective on the evolution of sexual size dimorphism. Trends Ecol. Evol. 17: 369-378

Barrio R, de Celis JF (2004) Regulation of spalt expression in the Drosophila wing blade in response to the Decapentaplegic signaling pathway. Proc Natl Acad Sci USA 101: 6021-6026. Doi: 10.1073/pnas.0401590101

Basler K, Struhl G (1994) Compartment boundaries and the control of Drosophila limb pattern by hedgehog protein. Nature 368: 208-214 
Beadle GW, Tatum EL, Clancy CW (1938) Food level in relation to rate of development and eye pigmentation in Drosophila melanogaster. Biol Bull 75: 447-462

Bennet-Clark HC, Dow M, Ewing AW, Manning A, von Schilcher F (1976) Courtship stimuli in Drosophila melanogaster. Behav Genetics 6: 93-95

Berg C, Rayner J (1995) The moment of inertia of bird wings and the inertial power requirement for flapping flight. J Exp Biol 198: 1655-1664

Bergmann C (1847) "Über die Verhältnisse der Wärmeökonomie der Thiere zu ihrer Grösse". Göttinger Studien 3: 595-708

Biddulph TA, Harrison JF (January 3-7, 2014) Oxygen modulates density effects on body size in Drosophila melanogaster. Society for Integrative and Comparative Biology, Austin, TX

Bitner-Mathé BC, Klaczko LB (1999) Plasticity of Drosophila melanogaster wing morphology: effect of sex, temperature and density. Genetica 105: 203-210

Blackman RK, Grimaila R, Koehler MM, Gelbart WM (1987) Mobilization of hobo elements residing within the decapentaplegic gene complex: suggestion of a new hybrid dysgenesis system in Drosophila melanogaster. Cell 49: 497-505

Blackman RK, Sanicola M, Raftery LA, Gillevet T, Gelbart WM (1991) An extensive 3' cis-regulatory region directs the imaginal disk expression of decapentaplegic, a member of the TGF- $\beta$ family in Drosophila. Development 111: 657-666

Boehm B, Westerberg H, Lesnicar-Pucko G, Raja S, Rautschka M, Cotterell J, Swoger J, Sharpe J (2010) The role of spatially controlled cell proliferation in limb bud morphogenesis. PLoS Biol 8: e1000420

Bollenbach, T., Pantazis P, Kicheva A, Bökel C, González-Gaitán M, Jülicher F (2008) Precision of the Dpp gradient. Development 135: 1137-1146

Bonduriansky R (2007) Sexual selection and allometry: a critical reappraisal of the evidence and ideas. Evolution 61: 838-849 
Bookstein FL (1991) Morphometric tools for landmark data: geometry and biology. Cambridge University Press, Cambridge

Bookstein F L (1996) Biometrics, biomathematics and the morphometric synthesis. Bull Math Biol 58: 313-365

Briceño RD, Ramos D, Eberhard WG (1996) Courtship behavior of male medflies (Ceratitis capitata; Diptera: Tephritidae) in captivity. Florida Entomol 79: 1-15

Briceño RD and Eberhard WG (2002) Decisions during courtship by male and female medflies, Ceratitis capitata (Diptera, Tephritidae): Correlated changes in male behavior and female acceptance criteria in mass-reared flies. Florida Entomol 85: 14-31

Briceño RD, Eberhard WG, Vilardi JC, Liedo P, Shelly TE (2002) Variation in the intermittent buzzing songs of male medflies (Diptera: Tephritidae) associated with geography, mass-rearing, and courtship success. Fla Entomol 85: 32-40

Brower DL (1986) engrailed gene expression in Drosophila imaginal discs. EMBO J 5: 2649-2656.

Bryan EH (1977) Morphometric adaptation of the housefly, Musca domestica L., in the United States. Evolution 31: 580-596

Bryant PJ (1975) Pattern formation in the imaginal wing disc of Drosophila melanogaster: Fate map, regeneration, and duplication. J Exp Zool 193: 49-78

Burk T, Webb JC (1983) Effect of male size on calling propensity, song parameters and mating success in Caribbean fruit flies, Anastrepha suspensa (Loew) (Diptera: Tephritidae). Ann Entomol Soc Am 76: 678-682

Butler MJ, Jacobsen TL, Cain DM, Jarman MG, Hubank M, Whittle JR, Phillips R, Simcox A (2003) Discovery of genes with highly restricted expression patterns in the Drosophila wing disc using DNA oligonucleotide microarrays. Development 130: 659-670 
Carter JR, Fraser TS, Fraser MJ Jr (2008) Examining the relative activity of several dicistrovirus intergenic internal ribosome entry site elements in uninfected insect and mammalian cell lines. J Gen Virol 89: 3150-3155. Doi: 10.1099/vir.0.2008/003921-0

Cavicchi S, Guerra D, Natali V, Pezzoli C, Giorgi G (1989) Temperature-related divergence in experimental populations of Drosophila melanogaster. II. Correlation between fitness and body dimensions. J Evol Biol 2: 235-251

Cavicchi S, Giorgi G, Natali V, Guerra D (1991) Temperature-related divergence in experimental populations of Drosophila melanogaster. III. Fourier and centroid analysis of wing shape and relationship between shape variation and fitness. J Evol Biol 4: 141-159

Cheverud JM (1982) Relationships among ontogenetic, static, and evolutionary allometry. Am J Phys Anthropol 59: 139-149

Cho SW, Kim S, Kim JM, Kim JS (2013) Targeted genome engineering in human cells with the Cas9 RNA-guided endonuclease. Nat Biotechnol 3: 230-232

Churchill-Stanland C, Stanland R, Wong TTY, Tanaka N, McInnis DO, Dowell R (1986) Size as a factor in the mating propensity of Mediterranean fruit flies, Ceratitis capitata (Diptera:Tephritidae), in the laboratory. J Econ Entomol 79: 614-618

Cohen B, McGuffin ME, Pfeifle C, Segal D, Cohen SM (1992) apterous, a gene required for imaginal disc development in Drosophila encodes a member of the LIM family of developmental regulatory proteins. Genes \& Dev 6: 715-729. Doi: 10.1101/gad.6.5.715

Cohen SM (1996) Controlling growth of the wing: vestigial integrates signals from the compartment boundaries. Bioessays 18: 855-858

Coleman KG, Poole SJ, Weir MP, Soeller WC, Kornberg T (1987). The invected gene of Drosophila: sequence analysis and expression studies reveal a close kinship to the engrailed gene. Genes Dev 1: 19-28 
Colless DH, McAlpine DK (1991) Diptera (flies). In Insects of Australia, vol. II (ed. Naumann ID, Carne PB, Lawrence JF, Nielsen ES, Spradberry JP, Taylor RW, Whitten MJ, Littlejohn MJ), pp. 717-786. Cornell University Press

Combes SA, Daniel TL (2003) Flexural stiffness in insect wings I. Scaling and the influence of wing venation. J Exp Biol 206: 2979-2987. Doi: 10.1242/jeb.00523

Comstock JH (1893) Evolution and Taxonomy. An essay on the application of the theory of natural selection in the classification of animals and plants, illustrated by a study of the evolution of the wings of insects. The Wilder Quarter-Century Book, Ithaca, New-York

Couso JP, Bate M, Martínez-Arias A (1993) A wingless-dependent polar coordinate system in Drosophila imaginal discs. Science 259: 484-489

Cowley DE, Atchley WR, Rutledge JJ (1986) Quantitative genetics of Drosophila melanogaster. I. Sexual dimorphism in genetic parameters for wing traits. Genetics 114: 549-566

Cowley DE, Atchley WR (1990) Development and quantitative genetics of correlation structure among body parts of Drosophila melanogaster. Am Nat 135: 242-268

Cowling DE and Burnet B (1981) Courtship songs and genetic control of their acoustic characteristics in sibling species of the Drosophila melanogaster subgroup. Anim Behav 29: 924935

Crick FHC, Lawrence PA (1975). Compartments and polyclones in insect development. Science 189: $340-347$

Crozatier M, Glise B, Vincent A (2004) Patterns in evolution: veins of the Drosophila wing. Trends Genet 20: 498-505

d'Assis Fonseca ECM (1968) Handbooks for the identification of british insects. Section (b) Muscidae. Royal Entomological Society of London. Volume 10, Part 4 (b) pp: 4-5

Dahmann C, Oates AC, Brand M (2011) Boundary formation and maintenance in tissue development. Nat Rev Genet 12: 43-55. Doi:10.1038/nrg2902 
David JR, Clavel M-F (1967) Influence de la temperature subie au cours du development sur divers characters biometriques des adultes de Drosophila melanogaster Meigen. J Insect Physiol 13: 717-729

Day SJ, Lawrence PA (2000) Measuring dimensions: the regulation of size and shape. Development 127: 2977-2987

de Camargo WR, de Camargo NF, Corrêa Ddo C, de Camargo AJ, Diniz IR (2015) Sexual dimorphism and allometric effects associated with the wing shape of seven moth species of Sphingidae (Lepidoptera: Bombycoidea). J Insect Sci 15: 107. Doi: http://dx.doi.org/10.1093/jisesa/iev083

de Moed GH, de Jong G, Schatloo W (1997) The phenotypic plasticity of wing size in Drosophila melanogaster: the cellular basis of its genetic variation. Heredity 79: 260-267

Debat V, Bégin M, Legout H, David JR (2003) Allometric and nonallometric components of Drosophila wing shape respond differently to developmental temperature. Evolution 57: 2773-2784

Demerec M (1950) Biology of Drosophila. New York: John Wiley and Sons, Inc.

Diamond SE, Kingsolver JG (2010) Environmental dependence of thermal reaction norms: host plant quality can reverse the temperature-size rule. Am Nat 175: 1-10

DiAngelo JR, Bland ML, Bambina S, Cherry S, Birnbaum MM (2009) The immune response attenuates growth and nutrient storage in Drosophila by reducing insulin signaling. Proc Natl Acad Sci USA 106: 20853-20858

Diaz-Benjumea FJ, Cohen SM (1993) Interaction between dorsal and ventral cells in the imaginal disc directs wing development in Drosophila. Cell 75: 741-752

DiNardo S, Kuner JM, Theis J, O'Farrell PH (1985) Development of embryonic pattern in $D$. melanogaster as revealed by accumulation of the nuclear engrailed protein. Cell 43: 59-69 
Dobzhansky TW (1929) Roux' Archiv f. Entwicklungsmechanik 115: 363. Doi: 10.1007/BF02078996

Doench JG, Fusi N, Sullender M, Hegde M, Vaimberg EW, Donovan KF, Smith I, Tothova Z, Wilen C, Orchard R, Virgin HW, Listgarten J, Root DE (2016) Optimized sgRNA design to maximize activity and minimize off-target effects of CRISPR-Cas9. Nat Biotechnol 34: 184-191. Doi: $10.1038 /$ nbt.3437

Drake AG, Klingenberg CP (2008) The pace of morphological change: historical transformation of skull shape in St Bernard dogs. Proc R Soc Lond B 275: 71-76

Dryden IL, Mardia KV (1998) Statistical shape analysis. John Wiley \& Sons, New York.

Edgar BA (2006) How flies get their size: genetics meets physiology. Nat Rev Genet 7: 907-916

Ejima A, Griffith LC (2007) Measurement of courtship behavior in Drosophila melanogaster. CSH Protoc: pdb.prot4847. Doi: 10.1101/pdb.prot 4847

Entchev EV, Schwabedissen A, González-Gaitán M (2000) Gradient formation of the TGF-beta homolog Dpp. Cell 103: 981-991

Esperk T, Tammaru T, Nylin S, Teder T (2007) Achieving high sexual size dimorphism in insects: females add instars. Ecol Entomol 32: 243-256. Doi: 10.1111/j.1365-2311.2007.00872.x

Ewing AW, Bennet-Clark HC (1968) The courtship songs of Drosophila. Behaviour 31: 288-301

Fairbairn DJ, Blanckenhorn WU, Székely T (2007) Sex, size and gender roles. Evolutionary studies of sexual size dimorphism. Oxford University Press, New York

Fisher B, Weiszmann R, Frise E, Hammonds A, Tomancak P, Beaton A, Berman B, Quan E, Shu S, Lewis S, Rubin G, Barale C, Laguertas E, Quinn J, Ghosh A, Hartenstein V, Ashburner M, Celniker S (2012). BDGP in situ homepage. http://insitu.fruitfly.org/cgi-bin/ex/insitu.pl

French V, Feast M, Partridge L (1998) Body size and cell size in Drosophila: the developmental response to temperature. J Insect Physiol 44: 1081-1089 
Fry SN, Sayaman R, Dickinson MH (2003) The aerodynamics of free-flight maneuvers in Drosophila. Science 300: 495-498

Garcia-Bellido A, Ripoll P, Morata G (1976) Developmental compartmentalization in the dorsal mesothoracic disc of Drosophila. Dev Biol 48: 132-147

Garcia-Bellido A, de Celis JF (1992) Developmental genetics of the venation pattern of Drosophila. Annu Rev Genet 26: 277-304

Gidaszewski NA, Baylac M, Klingenberg CP (2009) Evolution of sexual dimorphism of wing shape in the Drosophila melanogaster subgroup. BMC Evol Biol 9: 110. Doi: $10.1186 / 1471-2148-9-110$

Gilles AF, Schinko JB, Averof M (2015) Efficient CRISPR-mediated gene targeting and transgene replacement in the beetle Tribolium castaneum. Development 142: 2832-2839. Doi: $10.1242 /$ dev. 125054

Gleiser RM, Urrutia J, Gorla DE (2000) Body size variation of the floodwater mosquito Aedes albifasciatus in Central Argentina. Med Vet Entomol 14: 38-43

Gokhale RH, Shingleton AW (2015) Size control: the developmental physiology of body and organ size regulation. Wiley Interdiscip Rev Dev Biol 4: 335-356

Gong P, Epton MJ, Fu G, Scaife S, Hiscox A, Condon KC, Condon GC, Morrison NI, Kelly DW, Dafa'alla T, Coleman PG, Alphey L (2005) A dominant lethal genetic system for autocidal control of the Mediterranean fruitfly. Nat Biotechnol 23: 453-456. Doi: 10.1038/nbt1071

Good P (1994) Permutation tests: a practical guide to resampling methods for testing hypotheses. Springer, New York

Gould SJ (1966) Allometry and size in ontogeny and phylogeny. Biol Rev 41: 587-640

Gratz SJ, Cummings AM, Nguyen JN, Hamm DC, Donohue LK, Harrison MM, Wildonger J, O'Connor-Giles KM (2013) Genome engineering of Drosophila with the CRISPR RNA-guided Cas9 nuclease. Genetics 194: 1029-1035. Doi: 10.1534/genetics.113.152710 
Gratz SJ, Ukken FP, Rubinstein CD, Thiede G, Donohue LK, Cummings AM, O'Connor-Giles KM (2014) Highly specific and efficient CRISPR/Cas9-catalyzed homology-directed repair in Drosophila. Genetics 196: 961-971. Doi: 10.1534/genetics.113.160713

Green MR, Sambrook J (2012) Molecular cloning: a laboratory manual (Fourth Edition): Three-Volume Set (Avon, Mass.: Cold Spring Harbor Laboratory)

Guan K, Su J, Wang J, Yang Y (2015) Significance of hind wing morphology in distinguishing genera and species of cantharid beetles with a geometric morphometric analysis. ZooKeys 502: 11-25. Doi: 10.3897/zookeys.502.9191

Gumiel M, Catalá S, Noireau F, de Arias AR, Garcia A, Dujardin JP (2003) Wing geometry in Triatoma infestans (Klug) and T. melanosoma Martinez, Olmedo \& Carcavallo (Hemiptera: Reduviidae). Systematic Entomology 28: 173-179. Doi: 10.1046/j.1365-3113.2003.00206.x

Hadar N, Yaron S, Oren Z, Elly O, Itamar W, Johnathan G, Tama D, Offer G (2012) A screen identifying genes responsive to Dpp and Wg signaling in the Drosophila developing wing. Gene 494: 65-72. Doi 10.1016/j.gene.2011.11.047

Hale CR, Majumdar S, Elmore J, Pfister N, Compton M, Olson S, Resch AM, Glover CV 3rd, Graveley BR, Terns RM, Terns MP (2012) Essential features and rational design of CRISPR RNAs that function with the Cas RAMP module complex to cleave RNAs. Mol Cell 45: 292-302. Doi: 10.1016/j.molcel.2011.10.023

Hall JC (1994) The mating of a fly. Science 264: 1702-1714

Hama C, Ali Z, Kornberg TB (1990) Region-specific recombination and expression are directed by portions of the Drosophila engrailed promoter. Genes Dev 4: 1079-1093

Handler AM, James AA (2000) Insect Transgenesis: Methods and Applications, Boca Raton, FL: CRC Press LLC

Harmansa S, Hamaratoglu F, Affolter M, Caussinus E (2015) Dpp spreading is required for medial but not for lateral wing disc growth. Nature 527: 317-322. Doi: 10.1038/nature15712 
Hasty P, Rivera-Perez J, Bradley A (1991) The length of homology required for gene targeting in embryonic stem cells. Mol. Cell Biol 11: 5586-5591

Head G (1995) Selection on fecundity and variation in the degree of sexual size dimorphism among spider species (class Aranea). Evolution: 49: 776-781

Hediger M, Niessen M, Wimmer EA, Dübendorfer A, Bopp D (2001) Genetic transformation of the housefly Musca domestica with the lepidopteran derived transposon piggyBac. Insect Mol Biol 10: $113-119$

Hediger M, Burghardt G, Siegenthaler C, Buser N, Hilfiker-Kleiner D, Dübendorfer A, Bopp D (2004) Sex determination in Drosophila melanogaster and Musca domestica converges at the level of the terminal regulator doublesex. Dev Genes Evol 214: 29. Doi:10.1007/s00427-003-0372-2

Heller E, Fuchs E (2015) Tissue patterning and cellular mechanics. J Cell Biol 211: 219-231. Doi: $10.1083 /$ jcb. 201506106

Hernández G, Vázquez-Pianzola P, Sierra JM, Rivera-Pomar R (2004) Internal ribosome entry site drives cap-independent translation of reaper and heat shock protein 70 mRNAs in Drosophila embryos. RNA 10: 1783-1797. Doi: 10.1261/rna.7154104.

Hewitt CG (1914) The house-fly, Musca domestica Linn.: its structure, habits, development, relation to disease and control. Cambridge: University Press

Hisano Y, Sakuma T, Nakade S, Ohga R, Ota S, Okamoto H, Yamamoto T, Kawahara A (2015) Precise in-frame integration of exogenous DNA mediated by CRISPR/Cas9 system in zebrafish. Scientific Reports 5: 8841. Doi:10.1038/srep08841

Horn C, Wimmer EA (2000) A versatile vector set for animal transgenesis. Dev Genes Evol 210: $630-637$

Hornbruch A, Wolpert L (1970) Cell division in the early growth and morphogenesis of the chick limb. Nature 226: 764-766 
Hsu PD, Lander ES, Zhang F (2014) Development and applications of CRISPR-Cas9 for genome engineering. Cell 157: 1262-12711. Doi: 10.1016/j.cell.2014.05.010

Huang F, Dambly-Chaudière C, Ghysen A (1991) The emergence of sense organs in the wing disc of Drosophila. Development 111: 1087-1095

Huxley JS (1924) Constant differential growth-ratios and their significance. Nature 114: 895-896

Huxley JS (1932) Problems of relative growth. Reprinted 1993 edn. Johns Hopkins University Press, Baltimore

Huxley JS, Tessier G (1936) Terminology of relative growth. Nature 137: 780-781

Hwang WY, Fu Y, Reyon D, Maeder ML, Tsai SQ, Sander JD, Peterson RT, Yeh JR, Joung JK (2013) Efficient genome editing in zebrafish using a CRISPR-Cas system. Nat Biotechnol 3: $227-229$

Irish VF, Gelbart WM (1987) The decapentaplegic gene is required for dorsal-ventral patterning of the Drosophila embryo. Genes Dev 1: 868-879

Irvine KD, Wieschaus E. (1994) fringe, a Boundary-specific signaling molecule, mediates interactions between dorsal and ventral cells during Drosophila wing development. Cell 79: $595-606$

Jiang W, Zhou H, Bi H, Fromm M, Yang B, Weeks DP (2013) Demonstration of CRISPR/Cas9/sgRNA-mediated targeted gene modification in Arabidopsis, tobacco, sorghum and rice. Nucleic Acids Res 41: e188

Jinek M, Chylinski K, Fonfara I, Hauer M, Doudna JA, Charpentier E A (2012) Programmable dual-RNA-guided DNA endonuclease in adaptive bacterial immunity. Science 337: 816-821. Doi: $10.1126 /$ science. 1225829

Kacmarczyk T, Craddock EM (2000) Cell size is a factor in body size variation among Hawaiian and nonHawaiian species of Drosophila. Dros Inf Serv 83: 144-148 
Kammenga JE, Doroszuk A, Riksen JA, Hazendonk E, Spiridon L, Petrescu AJ, Tijsterman M, Plasterk RH, Bakker J (2007) A Caenorhabditis elegans wild-type defies the temperature-size rule owing to a single nucleotide polymorphism in tra-3. PLoS Genet 3: e34. Doi: 10.1371/journal.pgen.0030034

Kammer AE (1985) Flying. In Comprehensive Insect Physiology, Biochemistry and Pharmacology. Vol. 5, Nervous system: structure and motor function (ed. G. A. Kerkut and L. I. Gilbert), pp. 491-552. Oxford: Pergamon Press

Kears, M, Moir R, Wilson A, Stones-Havas S, Cheung M, Sturrock S, Buxton S, Cooper A, Markowitz S, Duran C et al. (2012). Geneious Basic: an integrated and extendable desktop software platform for the organization and analysis of sequence data. Bioinforma Oxf Engl 28: $1647-1649$

Khazaeli AA, Van Voorhies W, Curtsinger JW (2005) The relationship between life span and adult body size is highly strain-specific in Drosophila melanogaster. Exp Gerontol 40: 77-85

Kicheva A, Pantazis P, Bollenbach T, Kalaidzidis Y, Bittig T, Jülicher F, González-Gaitán M (2007) Kinetics of morphogen gradient formation. Science 315: 521-525. Doi: 10.1126/science.1135774

Kicheva A, González-Gaitán M (2008) The Decapentaplegic morphogen gradient: a precise definition. Curr Opin Cell Biol 20: 137-143

Kim J, Johnson K, Chen HJ, Carroll S, Laughon A (1997) Drosophila Mad binds to DNA and directly mediates activation of vestigial by Decapentaplegic. Nature 388: 304-308

Kingsolver JG and Huey RB (2008) Size, temperature and fitness: three rules. Evol Ecol Res 8: 703-715

Klein T (2001) Wing disc development in the fly: the early stages. Curr Opin Genet Dev 11: $470-475$

Klingenberg CP (1996) Multivariate allometry. In: Marcus LF, Corti M, Loy A, Naylor GJP, Slice DE (Eds) Advances in morphometrics. New York, Plenum Press, pp 23-49 
Klingenberg CP, McIntyre GS, Zaklan SD (1998) Left-right asymmetry of fly wings and the evolution of body axes. P Roy Soc Lond B Bio 265: 1255-1259

Klingenberg CP (2003) Developmental instability as a research tool: using patterns of fluctuating asymmetry to infer the developmental origins of morphological integration. In: Polak M. (Ed.) Developmental instability, causes of consequences. Oxford University Press, New York, 427-442

Klingenberg, CP (2008) MorphoJ Software/Documentation. Version 1.00i. Faculty of Life Sciences, University of Manchester, UK. http://www. flywings.org.uk/MorphoJ_page.htm

Klingenberg CP (2011) MorphoJ: an integrated software package for geometric morphometrics. Mol Ecol Resour 11: 353-357

Klingenberg CP (2016) Size, shape, and form: concepts of allometry in geometric morphometrics. Dev Genes Evol 226: 113. Doi:10.1007/s00427-016-0539-2

Kornberg T, Sidén I, O'Farrell P, Simon M (1985) The engrailed locus of Drosophila: in situ localization of transcripts reveals compartment-specific expression. Cell 40: 45-53

Koyama T, Rodrigues MA, Athanasiadis A, Shingleton AW, Mirth CK (2014) Nutritional control of body size through FoxO-Ultraspiracle mediated ecdysone biosynthesis. Elife 3: e03091. Doi: 10.7554/eLife.03091

Lavine L, Gotoh H, Brent CS, Dworkin I, Emlen DJ (2015) Exaggerated trait growth in insects. Annu Rev Entomol 60: 453-472

Lawrence PA, Morata G (1976) Compartments in the wing of Drosophila: A study of the engrailed gene. Dev Bio 50: 321-337. Doi: 10.1016/0012-1606(76)90155-X

Lecuit T, Cohen SM (1998) Dpp receptor levels contribute to shaping the Dpp morphogen gradient in the Drosophila wing imaginal disc. Development125: 4901-4907

Lee JJ, von Kessler DP, Parks S, Beachy PA (1992) Secretion and localized transcription suggest a role in positional signaling for products of the segmentation gene hedgehog. Cell 71: 33-50 
Lewis I, Held Jr (2005) Imaginal discs. The genetic and cellular logic of pattern formation. Cambridge University Press. pp 85-89

Liang G, Zhang H, Lou D, Yu D (2016) Selection of highly efficient sgRNAs for CRISPR/Cas9-based plant genome editing. Scientific Reports 6: 21451. Doi: 10.1038/srep21451

Lunde K, Biehs B, Nauber U, Bier E (1998) The knirps and knirps-related genes organize development of the second wing vein in Drosophila. Development 125: 4145-4154

Lunde K, Trimble JL, Guichard A, Guss KA, Nauber U, Bier E (2003) Activation of the knirps locus links patterning to morphogenesis of the second wing vein in Drosophila. Development 130: $235-248$

Lutz F (1948) Field Book of Insects. New York, NY: G. P. Putnam's Sons.

Madhavan MM, Schneiderman HA (1977) Histological analysis of dynamics of growth of imaginal discs and histoblast nests during larval development of Drosophila melanogaster. Roux's Arch Dev Biol 183: 269-305

Mali P, Yang L, Esvelt KM, Aach J, Guell M, DiCarlo JE, Norville JE, Church GM. (2013) RNA-guided human genome engineering via Cas9. Science 339: 823-826

Marcus JM (2001) The development and evolution of crossveins in insect wings. J Anat 199: $211-216$

Markow TA, O'Grady PM (2005) Evolutionary genetics of reproductive behavior in Drosophila. Annu Rev Genet 39: 263-291

Marty T, Muller B, Basler K, Affolter M (2000). Schnurri mediates Dpp-dependent repression of brinker transcription. Nat Cell Biol 2: 745-749. Doi: 10.1038/35036383

Masucci JD, Miltenberger RJ, Hoffmann FM (1990) Pattern-specific expression of the Drosophila decapentaplegic gene in imaginal disks is regulated by $3^{\prime}$ cis-regulatory elements. Genes Dev 4: 2011-2023 
Matamoro-Vidal A, Salazar-Ciudad I, Houle D (2015) Making quantitative morphological variation from basic developmental processes: Where are we? The case of the Drosophila wing. Dev Dyn. Doi: 10.1002/dvdy.24255

Miller RS, Thomas JL (1958) The effect of larval crowding and body size on the longevity of adult Drosophila melanogaster. Ecology 39: 118-125

Mirth CK, Shingleton AW (2012) Integrating body and organ size in Drosophila: recent advances and outstanding problems. Front Endocrinol 3: 49. Doi: 10.3389/fendo.2012.00049

Mirth CK, Tang HY, Makohon-Moore SC, Salhadar S, Gokhale RH, Warner RD, Koyama T, Riddiford LM, Shingleton AW (2014) Juvenile hormone regulates body size and perturbs insulin signaling in Drosophila. Proc Natl Acad Sci USA 111: 7018-7023

Mitrovski-Bogdanović A, Ivanovic A, Tomanovic Z, Zikic V, Stary P, Kavallieratos NG (2009) Sexual dimorphism in Ephedrus persicae (Hymenoptera: Braconidae: Aphidiinae): intraspecific variation in size and shape. Can Entomol 141: 550-560

Mitteroecker P, Gunz P (2009) Advances in geometric morphometrics. Evol Biol 36: 235-247

Mitteroecker P, Gunzz P, Windhager S, Schaefer K (2013) A brief review of shape, form, and allometry in geometric morphometrics, with applications to human facial morphology. Hystrix 24: 59-66. Doi: 10.4404/hystrix-24.1-6369

Monteiro LR (1999) Multivariate regression models and geometric morphometrics: the search for causal factors in the analysis of shape. Syst Biol 48: 192-199

Moraes EM, Manfrin MH, Laus AC, Rosada RS, Bomfin SC, Sene FM (2004) Wing shape heritability and morphological divergence of the sibling species Drosophila mercatorum and Drosophila paranaensis. Heredity 92: 466-473. Doi: 10.1038/sj.hdy.6800442

Morata G, Lawrence PA (1975) Control of compartment development by the engrailed gene in Drosophila. Nature 255: 614-617. Doi: 10.1038/255614a0 
Morata G (2001) How drosophila appendages develop. Nat Rev Mol Cell Biol 2: 89-97. Doi: $10.1038 / 35052047$

Müller B, Basler K (2000) The repressor and activator forms of Cubitus interruptus control Hedgehog target genes through common generic gli-binding sites. Development 127: 2999-3007

Murvosh CM, Fye RL, Labreque GC (1964) Studies on the mating behaviour of the house fly, Musca domestica. L. Ohio J Sci 64: 264-271

Navarro-Campos C, Martínez-Ferrer MT, Campos JM, Fibla JM, Alcaide J, Bargues L, Marzal C, Garcia-Marí F (2011) The influence of host fruit and temperature on the body size of adult Ceratitis capitata (Diptera: Tephritidae) under laboratory and field conditions. Environ Entomol 40: 931-938

Nellen D, R Burke, G Struhl, K Basler (1996) Direct and long-range action of a DPP morphogen gradient. Cell 85: 357-368

Neufeld TP, Edgart BA (1998) Connections between growth and the cell cycle. Curr Opin Cell Biol 10: 784-790

Neufeld TP, de la Cruz AF, Johnston LA, Edgar BA (1998) Coordination of growth and cell division in the Drosophila wing. Cell 93: 1183-1193

Ng M, Diaz-Benjumea FJ, Vincent JP, Wu J, Cohen SM (1996) Specification of the wing by localized expression of wingless protein. Nature 381:316-318

Nunney L, Cheung W (1997) The effect of temperature on body size and fecundity in female Drosophila melanogaster: evidence for adaptive plasticity. Evolution 51: 1529-1535

Oliveira MM, Shingleton AW, Mirth CK (2014) Coordination of wing and whole-body development at developmental milestones ensures robustness against environmental and physiological perturbations. PLoS Genet 10: e1004408. Doi: 10.1371/journal.pgen.1004408

Pantalouris EM (1957) Size response of developing Drosophila to temperature change. J Genet 55: $507-510$ 
Papanicolaou A, Schetelig MF, Arensburger P, Atkinson PW, Benoit JB, Bourtzis K, Castañera P, Cavanaugh JP, Chao H, Childers C, Curril I, Dinh H, Doddapaneni HV, Dolan A, Dugan S, Friedrich M, Gasperi G, Geib S, Georgakilas G, Gibbs RA, Giers SD, Gomulski LM, GonzálezGuzmán M, Guillem-Amat A, Han Y, Hatzigeorgiou AG, Hernández-Crespo P, Hughes DST, Jones JW, Karagkouni D, Koskinioti P, Lee SL, Malacrida AR, Manni M, Mathiopoulos K, Meccariello A, Murali SC, Murphy TD, Muzny DM, Oberhofer G, Ortego F, Paraskevopoulou MD, Poelchau M, Qu J, Reczko M, Robertson HM, Rosendale AJ, Rosselot AE, Saccone G, Salvemini M, Savini G, Schreiner P, Scolari F, Siciliano P, Sim SB, Tsiamis G, Ureña E, Vlachos IS, Werren JH, Wimmer EA, Worley KC, Zacharopoulou A, Richards S, Handler AM (2016) The whole genome sequence of the Mediterranean fruit fly, Ceratitis capitata (Wiedemann), reveals insights into the biology and adaptive evolution of a highly invasive pest species. Genome Biology 17: 192. Doi: 10.1186/s13059-016-1049-2

Partridge L, Ewing A, Chandler A (1987) Male size and mating success in Drosophila melanogaster: the role of male and female behaviour. Anim Behav 35: 555-562

Pechmann M (2011) Comparative studies of appendage and segment formation in arthropods. PhD Thesis. Georg-August-University Göttingen

Peck LS, Maddrell SHP (2005) Limitation of size by hypoxia in the fruit fly Drosophila melanogaster. J Exp Zool A Comp Exp Biol 303A: 968-975

Pétavy G, Morin JP, Moreteau B, David JR (1997) Growth temperature and phenotypic plasticity in two Drosophila sibling species: species: probable adaptive changes in flight capacities. J Evol Biol 10: 875-887

Pezzoli MC, Guerra D, Giorgi G, Garoia F, Cavicchi S (1997) Developmental constraints and wing shape variation in natural populations of Drosophila melanogaster. Heredity 79: 572-577

Pitman EJG (1937) Significance tests which may be applied to samples from any populations. II. The correlation coefficient test. J R Statist Soc B 4: 225-232

Pitnick S, Markow TA (1995) Delayed male maturity is a cost of producing large sperm in Drosophila Proc Natl AcadSci USA 92: 10614-10618 
Plowright RC, Stephen WP (1973) Evolutionary relationships in northern European Bombus and Psithyrus species (Apidae: Hymenoptera). Canadian Entomologist 105: 733-743. Doi: 10.4039/Ent105733-5

Poeck B, Hofbauer A, Pflugfelder GO (1993) Expression of the Drosophila optomotor-blind gene transcript in neuronal and glial cells of the developing nervous system. Development 117: 1017-1029

Port F, Bullock SL (2016) Augmenting CRISPR applications in Drosophila with tRNA-flanked sgRNAs. Nature Methods, AOP. Doi:10.1038/nMeth.3972

Porter JA, Von Kessler D, Ekker SC, Young KE, Lee JJ, Moses K, Beachy PA (1995) The product of hedgehog autoproteolytic cleavage active in local and long-range signalling. Nature 374: 363-366

Postlethwait JH (1978) Clonal analysis of Drosophila cuticular patterns. In The genetics and biology of Drosophila (ed. M. Ashburner and T.R.F. Wright), Academic Press, London, pp 359-441

Pretorius E (2005) Using geometric morphometrics to investigate wing dimorphism in males and females of Hymenoptera - a case study based on the genus Tachysphex Kohl (Hymenoptera: Sphecidae: Larrinae). Aust J Entomol 44: 113-121

Prudhomme JF, Rahola GN, Ouanaimi F, Guernaoui S, Boumezzough A, Bañuls A-L, Sereno D, Alten B (2012) Wing size and shape variation of Phlebotomus papatasi (Diptera: Psychodidae) populations from the south and north slopes of the Atlas Mountains in Morocco. J Vector Ecol 37: 137-147. Doi: 10.1111/j.1948-7134.2012.00210.x

R Development Core Team (2008) R: A language and environment for statistical computing. Vienna, Austria: R Foundation for Statistical Computing, http://www.R-project.org

Ran FA, Hsu PD, Wright J, Agarwala V, Scott DA, Zhang F (2013) Genome engineering using the CRISPR-Cas9 system. Nat Protoc 8: 2281-2308. Doi: 10.1038/nprot.2013.143 
Ray C (1960) The application of Bergmann's and Allen's rules to the poikilotherms. J Morphol 106: $85-108$

Ray RP, Nakata T, Henningsson P, Bomphrey RJ (2016) Enhanced flight performance by genetic manipulation of wing shape in Drosophila. Nat Commun. 7: 10851. Doi: 10.1038/ncomms10851

Restrepo S, Zartman JJ, Basler K (2014) Coordination of patterning and growth by the morphogen DPP. Curr Biol 24: 245-255. Doi: 10.1016/j.cub.2014.01.055.

Robinson SJW and Partridge L (2001) Temperature and clinal variation in larval growth efficiency in Drosophila melanogaster. J Evol Biol 14: 14-21

Rodríguez-Mendoza R, Muñoz M, Saborido-Rey F (2011) Ontogenetic allometry of the bluemouth, Helicolenus dactylopterus dactylopterus (Teleostei: Scorpaenidae), in the Northeast Atlantic and Mediterranean based on geometric morphometrics 670: 5. Doi: $10.1007 / \mathrm{s} 10750-011-0675-7$

Rohlf FJ (1990) Morphometrics. Annu Rev Ecol Syst 21: 299-316

Rohlf FJ. (1993) Relative warp analysis and an example of its application to mosquito wings. In: Marcus LF, Bello E, Garcia-Valdecasas A.(Eds) Contributions to Morphometrics. Museo Nacional de Ciencias Naturales, Marid, 264 pp

Rohlf FJ, Marcus LF. 1993. A revolution in morphometrics. Trends Ecol Evol 8: 129-132

Rohlf FJ (2004) tpsUtil, File Utility Program, Version 1.54. Department of Ecology and Evolution, State University of New York at Stony Brook

Rohlf FJ (2010) tpsDig, Digitize Landmarks and Outlines, Version 2.17. Department of Ecology and Evolution, State University of New York at Stony Brook

Rolli K (1976) Die akustischen Sexualsignale von Ceratitis capitata Wied. und Dacus olei Gmel. Z. Ang. Entomol. 81:219-223 
Romanova-Michaelides M, Aguilar-Hidalgo D, Jülicher F, González-Gaitán M (2015) The wing and the eye: a parsimonious theory for scaling and growth control? Wiley Interdiscip Rev Dev Biol 4: 591-608. Doi: 10.1002/wdev.195

Ruberte E, Marty T, Nellen D, Affolter M, Basler K (1995) An absolute requirement for both the type II and type I receptors, Punt and Thick veins, for Dpp signaling in vivo. Cell 80: 889-897

Rybak F, Aubin T, Moulin B, Jallon JM (2002) Acoustic communication in Drosophila melanogaster courtship: Are pulse- and sine-song frequencies important for courtship success? Canadian J Zool 80: 987-996

Sadeghi S, Dumont HJ (2014) Variation in the shape of the wings and taxonomy of Eurasian populations of the Calopteryx splendens complex (Odonata: Calopterygidae). Eur J Entomol 111: 575-583. Doi: 10.14411/eje.2014.073

Sander K (1976) Specification of the basic body pattern in insect embryogenesis. Adv Insect Physiol 12: 125-238

Santos M, Fowler K, Partridge L (1994) Gene-environment interaction for body size and larval density in Drosophila melanogaster: an investigation of effects on development time, thorax length and adult sex ratio. Heredity $72: 515-521$

Sato M, Saigo K (2000) Involvement of pannier and u-shaped in regulation of decapentaplegic-dependent wingless expression in developing Drosophila notum. Mech Dev 93: $127-138$

Schachter-Broide J, Gürtler RE, Kitron U, Dujardin J-P (2009) Temporal variations of wing size and shape of Triatoma infestans (Hemiptera: Reduviidae) populations from northwestern Argentina using geometric morphometry. J Med Entomol 46: 994-1000

Scheiner SM, Lyman RF (1989) The genetics of phenotypic plasticity. Heritability. J Evol Biol 2: 95-107 
Scheiner SM, Lyman RF (1991) The genetics of phenotypic plasticity II. Response to selection. J Evol Biol 3: 23-50

Schlichting CD, Pigliucci M (1999) Phentypic evolution - a reaction norm perspective. Heredity 82: $344-344$

Schwank G, Basler K (2010) Regulation of organ growth by morphogen gradients. Cold Spring Harb Perspect Biol 2: a001669. Doi: 10.1101/cshperspect.a001669

Scolari F, Schetelig MF, Bertin S, Malacrida AR, Gasperi G, Wimmer EA (2008) Fluorescent sperm marking to improve the fight against the pest insect Ceratitis capitata (Wiedemann; Diptera: Tephritidae). N Biotechnol 25: 76-84. Doi: 10.1016/j.nbt.2008.02.001

Scott JG, Warren WC, Beukeboom LW, Bopp D, Clark AG, Giers SD, Hediger M, Jones AK, Kasai S, Leichter CA, Li M, Meisel RP, Minx P, Murphy TD, Nelson DR, Reid WR, Rinkevich FD, Robertson HM, Sackton TB, Sattelle DB, Thibaud-Nissen F, Tomlinson C, van de Zande L, Walden KKO, Wilson RK, Liu N (2014) Genome of the house fly, Musca domestica L., a global vector of diseases with adaptations to a septic environment. Genome Biology 15: 466. Doi: $10.1186 / \mathrm{s} 13059-014-0466-3$

Shen J, Dorner C, Bahlo A, Pflugfelder GO (2008) optomotor-blind suppresses instability at the A/P compartment boundary of the Drosophila wing. Mech Dev 125: 233-246. Doi: 10.1016/j.mod.2007.11.006

Sheng G, Thouvenot E, Schmucker D, Wilson DS, Desplan C (1997) Direct regulation of rhodopsin 1 by Pax-6/eyeless in Drosophila: evidence for a conserved function in photoreceptors. Genes \& Dev 11: 1122-1131. Doi: 10.1101/gad.11.9.1122

Shine R (1979) Sexual selection and sexual dimorphism in the Amphibia. Copeia 2: 297-306

Shine R (1994) Sexual size dimorphism in snakes revisited. Copeia 2: 326-346

Shingleton AW, Mirth CK, Bates PW (2008) Developmental model of static allometry in holometabolous insects. Proc R Soc B 275: 1875-1885 
Shingleton AW, Estep CM, Driscoll MV, Dworkin I (2009) Many ways to be small: different environmental regulators of size generate distinct scaling relationships in Drosophila melanogaster. Proc R Soc B 276: 2625-2633

Shyy W, H Aono, C Kang, H Liu (2013) An Introduction to flapping wing aerodynamics. Part of Cambridge Aerospace Series

Simpson P (2007) The stars and stripes of animal bodies: evolution of regulatory elements mediating pigment and bristle patterns in Drosophila. Trends in Genetics 23: 350-358

Siomava N, Wimmer EA, Posnien N (2016) Size relationships of different body parts in the three dipteran species Drosophila melanogaster, Ceratitis capitata and Musca domestica. Dev Genes Evol 226: 245-256. Doi: 10.1007/s00427-016-0543-6

Sivinski J, Burk T, Webb JC (1984) Acoustic courtship signals in the Caribbean fruit fly, Anastrepha suspensa (Loew). Anim Behav 32: 1011-1016

Slice DE (2005) Modern morphometrics. In: Slice DE, editor. Modern morphometrics in physical anthropology. New York: Kluwer Academic/Plenum Publishers. pp 1-45

Smithies O, Gregg RG, Boggs SS, Koralewski MA, Kucherlapati RS (1985) Insertion of DNA sequences into the human chromosomal-globin locus by homologous recombination. Nature 317 : $230-234$

Spieth HT (1952) Mating behaviour within the genus Drosophila (Diptera). Bull Am Mus Nat Hist 99: 401-474

St Johnston RD, Hoffmann FM, Blackman RK, Segal D, Grimaila R, Padgett RW, Irick HA, Gelbart WM (1990) Molecular organization of the decapentaplegic gene in Drosophila melanogaster. Genes Dev 4: 1114-27

Starmer WT, Wolf LL (1989) Causes of variation in wing loading among Drosophila species. Biol J Linn Soc 37: 247-261 
StatSoft Inc (2002) STATISTICA for Windows (Computer program manual). http://www.statsoft.com

Stillwell RC, Blanckenhorn WU, Teder T, Davidowitz G, Fox CW (2010) Sex differences in phenotypic plasticity affect variation in sexual size dimorphism in insects: from physiology to evolution. Annu Rev Entomol 55: 227-245

Stillwell RC, Dworkin I, Shingleton AW, Frankino WA (2011) Experimental manipulation of body size to estimate morphological scaling relationships in Drosophila. J Vis Exp 56: 3162.

Stoneley M, Willis AE (2004) Cellular internal ribosome entry segments: structures, trans-acting factors and regulation of gene expression. Oncogene 23: 3200-3207. Doi:10.1038/sj.onc.1207551

Swarup S, Verheyen EM (2012) Wnt/Wingless signaling in Drosophila. Cold Spring Harb Perspect Biol 4: pii: a007930. Doi: 10.1101/cshperspect.a007930

Tabata T, Eaton S, Kornberg TB (1992) The Drosophila hedgehog gene is expressed specifically in posterior compartment cells and is a target of engrailed regulation. Genes Dev 6: 2635-2645

Tabata T, Kornberg TB (1994) Hedgehog is a signaling protein with a key role in patterning Drosophila imaginal discs. Cell 76: 89-102

Talyn BC, Dowse HB. (2004) The role of courtship song in sexual selection and species recognition by female Drosophila melanogaster. Anim Behav 68: 1165-1180

Tanimoto H, Itoh S, ten Dijke P, Tabata T (2000) Hedgehog creates a gradient of DPP activity in Drosophila wing imaginal discs. Mol Cell 5: 59-71

Tashiro S, Michiue T, Higashijima S, Zenno S, Ishimaru S, Takahashi F, Orihara M, Kojima T, Saigo K (1993) Structure and expression of hedgehog, a Drosophila segment-polarity gene required for cell-cell communication. Gene124: 183-189

Teder T, Tammaru T (2005) Sexual size dimorphism within species increases with body size in insects. Oikos 108: 321-334 
Teleman AA, Cohen SM (2000) Dpp gradient formation in the Drosophila wing imaginal disc. Cell 103: 971-80

Tennekes H (2009) The simple science of flight: from insects to jumbo jets. - Rev. and expanded ed. Cambridge, Massachusetts: The MIT Press

Testa ND, Dworkin I (2016) The sex-limited effects of mutations in the EGFR and TGF- $\beta$ signaling pathways on shape and size sexual dimorphism and allometry in the Drosophila wing. Dev Genes Evol 226: 159-71. Doi: 10.1007/s00427-016-0534-7

Thomas KR, Folger KR, Capecchi MR (1986) High frequency targeting of genes to specific sites in the mammalian genome. Cell 44: 419-428

Thompson DW (1917) Growth and form. Cambridge: Cambridge University Press. pp 793

Tickle C, Summerbell D, Wolpert LP (1975) Positional signaling and specification of digits in chick limb morphogenesis. Nature 254: 199-202

Tomoyasu Y, Ueno N, Nakamura M (2000) The decapentaplegic morphogen gradient regulates the notal wingless expression through induction of pannier and $u$-shaped in Drosophila. Mech Dev 96: $37-49$

Torres A, Miranda-Esquivel DR (2016) Wing shape variation in the taxonomic recognition of species of Diachlorus Osten-Sacken (Diptera: Tabanidae) from Colombia. Neotrop Entomol 45: 180. Doi: $10.1007 / \mathrm{s} 13744-015-0350-1$

Tsuneizumi K, Nakayama T, Kamoshida Y, Kornberg TB, Christian JL, Tabata T (1997) Daughters against dpp modulates $d p p$ organizing activity in Drosophila wing development. Nature 389: 627-31

StatSoft, Inc. (1997). Electronic Statistics Textbook. Tulsa, OK: StatSoft. WEB: http://www.statsoft.com/textbook/stathome.html.

Umulis DM, Othmer HG (2013) Mechanisms of scaling in pattern formation. Development 140: 4830-4843. Doi: 10.1242/dev.100511 
Viscosi V, Cardini A (2011) Leaf morphology, taxonomy and geometric morphometrics: a simplified protocol for beginners. PLoS ONE 6: e25630. Doi: 10.1371/journal.pone.0025630

Wartlick O, González-Gaitán M (2011) The missing link: implementation of morphogenetic growth control on the cellular and molecular level. Curr Opin Genet Dev 21: 690-695.

Wartlick O, Mumcu P, Jülicher F, González-Gaitán M (2011a) Understanding morphogenetic growth control - lessons from flies. Nat Rev Mol Cell Biol 1: 594-604. Doi: 10.1038/nrm3169

Wartlick O, Mumcu P, Kicheva A, Bittig T, Seum C, Jülicher F, González-Gaitán M (2011b) Dynamic of Dpp signaling and proliferation control. Science 331: 1154-1159. Doi: $10.1126 /$ science. 1200037

Webb JC, Calkins CO, Chambers DL, Schwienbacher W, Russ K (1983) Acoustical aspects of behavior of mediterranean fruit fly, Ceratitis capitata: analysis and identification of courtship sounds. Entomol Exp Applic 33: 1-8

Webb JC, Sivinski J, Litzkow C (1984) Acoustical behaviour and sexual success in Caribbean fruit fly, Anastrepha suspensa (Loew) (Diptera: Tephritidae). Environ Entomol 13: 650-656

Weigmann K, Cohen SM, Lehner CF (1997) Cell cycle progression, growth and patterning in imaginal discs despite inhibition of cell division after inactivation of Drosophila Cdc2 kinase. Development 124: 3555-3563

Wharton KA, Ray RP, Gelbart WM (1993) An activity gradient of decapentaplegic is necessary for the specification of dorsal pattern elements in the Drosophila embryo. Development 117: 807-822

Wheeler DA, Fields WL, Hall JC (1988) Spectral analysis of Drosophila courtship songs: D. melanogaster, D. simulans, and their interspecific hybrid. Behavior Genetics 18: 675-703

Wiedenheft B, Sternberg SH, Doudna JA (2012) RNA guided genetic silencing systems in bacteria and archaea. Nature 7385: 331-338

Wiegmann BM, Trautwein MD, Winkler IS, Barr NB, Kim J-W, Lambkin C, Bertone MA, Cassel BK, Bayless KM, Heimberg AM, Wheeler BM, Peterson KJ, Pape T, Sinclair BJ, Skevington JH, 
Blagoderov V, Caravas J, Kutty SN, Schmidt-Ott U, Kampmeier GE, Thompson FC, Grimaldi DA, Beckenbach AT, Courtney GW, Friedrich M, Meier R, Yeates DK (2011) Episodic radiations in the fly tree of life. PNAS 108: 5690-5695

Wilkinson GS, Johns PM (2005) Sexual selection and the evolution of mating systems in flies. In: Wiegmann BM, Yeates DK (Eds.) The Evolutionary Biology of Flies. Columbia University Press, New York, pp. 312-329

Williams JA, Paddock SW, Carroll SB (1993) Pattern formation in a secondary field: a hierarchy of regulatory genes subdivides the developing Drosophila wing disc into discrete subregions. Development 117: 571-584

Wolpert L (1969) Positional information and the spatial pattern of cellular differentiation. J Theor Biol $25: 1-47$

Wootton RJ, Herbert RC, Young PG, Evans KE (2003) Approaches to the structural modelling of insect wings. Philos Trans R Soc Lond B Biol Sci 358: 1577-1587. Doi: 10.1098/rstb.2003.1351

Wülbeck C, Simpson P. (2000) Expression of achaete-scute homologues in discrete proneural clusters on the developing notum of the medfly Ceratitis capitata, suggests a common origin for the stereotyped bristle patterns of higher Diptera. Development 127: 1411-1420

Yang M, Hatton-Ellis E, Simpson P (2012) The kinase Sgg modulates temporal development of macrochaetes in Drosophila by phosphorylation of Scute and Pannier. Development 139: 325-334.

Yeaman S, Chen Y, Whitlock MC (2010) No effect of environmental heterogeneity on the maintenance of genetic variation in wing shape in Drosophila melanogaster. Evolution 64: 33983408. Doi: $10.1111 / j .1558-5646.2010 .01075 . x$

Yoshiyama M, Kimura K, Honda H (2000) Technique for microinjection into eggs of the housefly, Musca domestica (Diptera: Muscidae). Appl Entomol Zool 35: 27-30

Zecca M, Basler K, Struhl G (1995) Sequential organizing activities of engrailed, hedgehog and decapentaplegic in the Drosophila wing. Development 121: 2265-2278 


\section{ACKNOWLEDGMENTS}

I thank Yanli Wu, Dr. Leo Beukeboom, and Dr. Daniel Bopp for providing Musca flies, Yanli $\mathrm{Wu}$ for sharing gDNA as well as Hassan Mutasim Mohammed Ahmed and Mohammad Karami Nejad Ranjbar for primers and advise on cloning strategies. Additional thanks to Dr. Daniel Bopp, Svenia Dilara Heinze, and Tea Kohlbrenner for their hospitality during my visit to the laboratory in the Institute of Molecular Life Sciences, University of Zürich. I thank my supervisor Dr. Ernst A. Wimmer and my Thesis Committee members Dr. Andreas Wodarz and Dr. Ronald P. Kühnlein for the support and helpful discussions during the project. Preparing and writing of manuscripts based on this project was supported by Dr. Nico Posnien. I thank him for his help and bright ideas that helped me to improve the obtained results.

This work was supported by the German Academic Exchange Service (DAAD, Deutscher Akademischer Austauschdienst) fellowship number 91540915 to Natalia Siomava. Additional financial support was received from the Gottingen Graduate School for Neurosciences, Biophysics and Molecular Biosciences (GGNB) and the Volkswagen Foundation (project number: 85 983; to Dr. Nico Posnien).

The last but not least, I want to thank to my husband, Dr. Michael Siomau. I am very grateful for his help and support during the whole period of my $\mathrm{PhD}$, for his patience in any situation, and understanding. I appreciate it very much and I will always remember it. 
132 | ACKNOWLEDGMENTS 


\section{APPENDIX}

\subsection{Abbreviations}

\begin{tabular}{|c|c|}
\hline $\mathrm{AA}$ & amino acids \\
\hline ANCOVA & analysis of covariance \\
\hline AP & anteroposterior \\
\hline BLAST & Basic Local Alignment Search Tool \\
\hline BSC & body size coefficient \\
\hline bp & base pair \\
\hline cDNA & complementary DNA \\
\hline CDS & coding DNA sequence \\
\hline CNS & central nervous system \\
\hline CRISPR & clustered regularly interspaced short palindromic repeats \\
\hline CVA & canonical variate analysis \\
\hline DFA & discriminant function analysis \\
\hline DIG & digoxigenin \\
\hline DV & dorsoventral \\
\hline GPA & generalized Procrustes analysis \\
\hline gRNA & guide RNA \\
\hline HDR & homology directed repair \\
\hline IRES & internal ribosome entry site \\
\hline ISH & in situ hybridization \\
\hline $\mathrm{kb}$ & kilobase \\
\hline $\mathrm{mcm}$ & micrometer \\
\hline Mdn & median \\
\hline $\mathrm{mm}$ & millimetre \\
\hline $\mathrm{mM}$ & millimolar \\
\hline mRNA & messenger RNA \\
\hline NCBI & National Center for Biotechnology Information \\
\hline
\end{tabular}


NS

OFR

PAM protospacer-adjacent motif

PBS phosphate-buffered saline

PC1 Principal Component 1

PCA principal component analysis

PCR polymerase chain reaction

PFA paraformaldehyde

PL pupal length

PNS peripheral nervous system

PV pupal volume

PW pupal width

RACE rapid amplification of cDNA ends

$\mathrm{RH}$ relative humidity

RT room temperature $\left(22 \pm 2^{\circ} \mathrm{C}\right)$

sgRNA single guide RNA

SNP single nucleotide polymorphism

SSD sexual size dimorphism

SShD sexual shape dimorphism

TPS thin-plate spline

UPM universal primer mix

UTR untranslated region

WA wing area

WCS wing centroid size 


\section{Genes and proteins:}

$\begin{array}{ll}\text { ap (Ap) } & \text { apterous gene (Apterous protein) } \\ \text { dad (Dad) } & \text { daughters against Dpp gene (Daughters against Dpp protein) } \\ \text { dpp (Dpp) } & \text { decapentaplegic gene (Decapentaplegic protein) } \\ \text { en }(\mathrm{En}) & \text { engrailed gene (Engrailed protein) } \\ \text { hh }(\mathrm{Hh}) & \text { hedgehog gene (Hedgehog protein) } \\ \text { kni (Kni) } & \text { knirps gene (Knirps protein) } \\ \text { omb (Omb) } & \text { optomotor-blind gene (Optomotor-blind protein) } \\ \text { salm (Spalt) } & \text { spalt major gene (Spalt major protein) } \\ \text { wg (Wg) } & \text { wingless gene (Wingless protein) } \\ \text { Cas } & \text { CRISPR associated protein } \\ \text { DsRed } & \text { Discosoma species red fluorescent protein } \\ \text { ECFP } & \text { enhanced Cyan fluorescent protein } \\ \text { EGFP } & \text { enhanced Green fluorescent protein } \\ \text { GFP } & \text { Green fluorescent protein } \\ \text { Mad } & \text { Mather against Dpp } \\ \text { FP } & \text { fluorescent protein } \\ \text { Tkv } & \text { Thickveins protein }\end{array}$




\subsection{Estimation of wing size}

Table 11.1: Number of bristles along the width line in Drosophila and Ceratitis wings. N (area) shows the total number of bristles in the defined area, number of rows is shown separately for each quadrant of the wing, $\mathrm{N}$ (row) is a computed number of bristles in one row along the wing width line.

\begin{tabular}{|c|c|c|c|c|c|c|c|}
\hline \multirow{2}{*}{ Name } & \multirow{2}{*}{ Side } & \multirow{2}{*}{$\mathbf{N}$ (area) } & \multicolumn{3}{|c|}{ Number of rows } & \multirow{2}{*}{$\mathbf{N}($ row $)$} & \multirow{2}{*}{$\begin{array}{c}\text { Wing width, } \\
\text { mcm }\end{array}$} \\
\hline & & & Top & Middle & Bottom & & \\
\hline \multirow{2}{*}{ Cc28025fSEx1-06 } & left & 512 & 4.5 & 3.5 & 3 & 140 & 1656 \\
\hline & right & 512 & 3.5 & 3.5 & 3 & 154 & 1627 \\
\hline \multirow{2}{*}{ Cc28025fSEx1-08 } & left & 504 & 4 & 3 & 3.5 & 144 & 1632 \\
\hline & right & 508 & 3.5 & 3 & 3.5 & 153 & 1636 \\
\hline \multirow{2}{*}{ Cc28025fSEx1-09 } & left & 538 & 4 & 4 & 3 & 147 & 1653 \\
\hline & right & 523 & 4 & 4 & 3 & 143 & 1620 \\
\hline \multirow{2}{*}{ Cc28025fSEx1-11 } & left & 505 & 4 & 3 & 3 & 152 & 1620 \\
\hline & right & 500 & 4 & 3 & 3.5 & 143 & 1618 \\
\hline \multirow{2}{*}{ Cc28025fSEx1-12 } & left & 511 & 4 & 3.5 & 3.5 & 140 & 1631 \\
\hline & right & 522 & 4.5 & 4 & 3 & 136 & 1637 \\
\hline \multirow{2}{*}{ Dr25025fSEx1-15 } & left & 338 & 3 & 3 & 2.5 & 119 & 1035 \\
\hline & right & 342 & 3 & 2.5 & 3 & 121 & 1035 \\
\hline \multirow{2}{*}{ Dr25025fSEx1-16 } & left & 320 & 3 & 2.5 & 2 & 128 & 1044 \\
\hline & right & 321 & 2.5 & 2.5 & 3 & 121 & 1023 \\
\hline \multirow{2}{*}{ Dr25025fSEx1-17 } & left & 278 & 2 & 2.5 & 2.5 & 119 & 1033 \\
\hline & right & 297 & 2.5 & 2.5 & 2 & 127 & 1037 \\
\hline \multirow{2}{*}{ Dr25025fSEx1-18 } & left & 376 & 3 & 2.5 & 3 & 133 & 1051 \\
\hline & right & 335 & 3 & 2.5 & 2.5 & 125 & 1047 \\
\hline \multirow{2}{*}{ Dr25025fSEx1-19 } & left & 338 & 2.5 & 2.5 & 3 & 127 & 1026 \\
\hline & right & 329 & 3 & 3 & 2 & 124 & 1020 \\
\hline \multirow{2}{*}{ Dr25025fSEx1-20 } & left & 357 & 2.5 & 3 & 3 & 126 & 1071 \\
\hline & right & 343 & 2.5 & 3 & 3 & 121 & 1055 \\
\hline
\end{tabular}




\subsection{Primers used in this project}

Table 11.2: Primers for RACE-PCR. Primer names, sequences, gene names, and species are shown.

\begin{tabular}{|c|c|c|}
\hline Name & Sequence 5'->3' & Gene, species \\
\hline C-dpp -R & CGCCAGCACTGCGAGGAGTAGAAGCC & \multirow{2}{*}{ dpp, Ceratitis } \\
\hline C-dpp-F & AAGTGCCAAAGGCCTGCTGTGTGCC & \\
\hline C-wg-R & TGTGTGACAGCGGCACTGGTGATGGC & \multirow{2}{*}{$w g$, Ceratitis } \\
\hline C-wg-F & TGCAGCCAGCCCGAATGCTTTTAGTGC & \\
\hline C-en-R & TGCGGTAGGCCAGGCGGACTTGGAG & \multirow{2}{*}{ en, Ceratitis } \\
\hline C-en-F & CCGCGTACCGCCTTCTCCAGCGAAC & \\
\hline C-ap-R & ACCGACCGCAACCAGCGCAATCTTC & \multirow{2}{*}{ ap, Ceratitis } \\
\hline C-ap-F & GGTGGCGGTAGCGGTTCCCCTCAAC & \\
\hline C-hh-F & CGATATCCGCTGCGACGACGCCCAC & hh, Ceratitis \\
\hline C-salm-F & CCAATCACGATCCACCACC & \multirow{2}{*}{ salm, Ceratitis } \\
\hline C-salm-R & CAGCTTGTTTGACCACTGGC & \\
\hline C-omb_v.1-R & GCGGCGCCCTCTTTCGGACTACCC & \multirow{4}{*}{ omb, Ceratitis } \\
\hline C-omb_v.1-F & CCTTTGATGCCGTCACGCCCGGCTC & \\
\hline C-omb_v.2-R & CTCGGTCGGAGTGCTGACACATGC & \\
\hline C-omb_v.2-F & CTGTGCCGGGTCATCACGCGTTGGG & \\
\hline C-kni-R & TGGAACGACGGCCATAGCGCGAACC & \multirow{2}{*}{ kni, Ceratitis } \\
\hline C-kni-F & CAGCATCAACGGGGTCGGCGGTGTC & \\
\hline C-mad-R & TCTGGACATGGCGCTGCTGGAAGTGG & \multirow{2}{*}{ mad, Ceratitis } \\
\hline C-mad-F & TGGGCTCGCCGCATAACGCCATCAG & \\
\hline C-mad_3-R & CTCGGACCACTTGCCCTCGACCGTG & \multirow{2}{*}{ mad-3like, Ceratitis } \\
\hline C-mad_3-F & TGGTTGATGAAAAGTAGCGAGGCGCAGG & \\
\hline C-mad_4-R & CACCACCACCACCTGGCAAGCCCAC & \multirow{2}{*}{ mad-4like, Ceratitis } \\
\hline C-mad_4-F & CGGCTGGTCCGTTAGTAGGCGGGATGG & \\
\hline C-mad_7-R & GCGACTCCAGATTGCTGTTCCCATCG & \multirow{2}{*}{ mad-7like, Ceratitis } \\
\hline C-mad_7-F & CGACCGCGTTGGCAATCGTTGCTGTG & \\
\hline M-wg-F & GGTGGTCGTGGTGGAGCGTTGTGCC & wg, Musca \\
\hline M-en-F & CAAAGTCCCCAGCCCATCCCGCCAC & en, Musca \\
\hline M-hh-R & GACGCCCGATGCTCCCAGGGTCTG & \multirow{2}{*}{ hh, Musca } \\
\hline M-hh-F & TGTGGTCAACTCTGTGGCTGCCTCC & \\
\hline M-mad-F & TGGACCGCTGCAGTGGCTGGATAAGG & mad, Musca \\
\hline M-mad_3-R & CAGTGGGTTTGCTGCGCGGTACGG & \multirow{2}{*}{ mad-3like, Musca } \\
\hline M-mad_3-F & TCGCAGTCCGTGTCGCAGGGTTTCG & \\
\hline M-mad_6-R & GCGACGAATTGTTGTCACCGTTGTGGC & \multirow{2}{*}{ mad-6like, Musca } \\
\hline M-mad_6-F & GGCTACATACGACTCCAGACACCCG & \\
\hline M-kni-F & CCACACCGGAACCCGCCACCTTTGC & kni, Musca \\
\hline M-omb_v.1-F & ACCCACCACGCCGCCCTCCCTTATG & \multirow{2}{*}{$o m b, M u s c a$} \\
\hline M-omb_v.2-R & GCCTCAGCGGTGGCCCATCAACTGC & \\
\hline UPM-long & $\begin{array}{l}\text { CTAATACGACTCACTATAGGGCAAGCAG } \\
\text { TGGTATCAACGCAGAGT }\end{array}$ & \multirow{2}{*}{$\begin{array}{c}\text { All genes, } \\
\text { Ceratitis and } \mathrm{Musca}\end{array}$} \\
\hline UPM-short & CTAATACGACTCACTATAGGGC & \\
\hline
\end{tabular}


Table 11.3: Primers used for cloning of gene fragments. Primer names, sequences, gene names, and species are shown.

\begin{tabular}{|c|c|c|}
\hline Name & Sequence 5'->3' & Target gene, species \\
\hline Cc-omb_v.2-Fsp & GCCTCAGCTCCGTAAAAGC & \multirow{4}{*}{ omb v.2,Ceratitis } \\
\hline Cc-omb_v.2-Fsp2 & CATGTGTCAGCACTCCGAC & \\
\hline Cc-omb_v.2-Fsp3 & CAGAAGGTCGTCTCATTTCAC & \\
\hline Cc-omb_v.2-Rsp & GGTAAACAGAGAAGACGTACAG & \\
\hline Cc-omb_v.1-Rsp & GGTATTTCATTTGCGTATGCGC & \multirow{3}{*}{ omb v.1,Ceratitis } \\
\hline Cc-omb_v.1-Rsp2 & GGACCCGCGATTCGACATGAGCGC & \\
\hline Cc-omb_v.1-Rsp3 & ACATGTGTAGGATTGAGTTTGTC & \\
\hline Md-omb_v.1-R & CATTGATCTGACGCCAGGC & \multirow{2}{*}{ omb, Musca } \\
\hline Md-omb_v.1-F & GATCCGGAAATGCCCAAACG & \\
\hline Md-dpp-F & GGTCCACGCACCTATAGC & \multirow{2}{*}{$d p p, M u s c a$} \\
\hline Md-dpp-R & CCACAACCCACCACTGTC & \\
\hline Md-mad_4-F & CATTTGCTGCGCCTCTGAAC & \multirow{2}{*}{ mad-4like, Musca } \\
\hline Md-mad_4-R & GGCACCATCCACGAACTAC & \\
\hline Md-omb_v.2-F & GTCGGCTTTACTGAGTGCC & \multirow{2}{*}{ omb, Musca } \\
\hline Md-omb_v.2-R & GGATGTACATGCGTTTGGGC & \\
\hline M-ap-R & CATGCGACGCCATTTGGCACGGGC & \multirow{2}{*}{ ap, Musca } \\
\hline M-ap-F & GTCCAACACCACCTGTAGCACATCAAGG & \\
\hline Md-kni-F & GAAATGATGAACCAAACGTGC & \multirow{2}{*}{ kni, Musca } \\
\hline Md-kni-R & TTGTTGGTGCAGGGGCAG & \\
\hline Cc-kni-full-F & CTGCCAGTTCTCGAAGCG & \multirow{2}{*}{ kni, Ceratitis } \\
\hline Cc-kni-full-R & TGGTATCAACGCAGAGTGC & \\
\hline Cc-salm_new-F & CCTCCGAGACACTGAAGC & \multirow{2}{*}{$\operatorname{salm}($ new), Ceratitis } \\
\hline Cc-salm_new-R & CCCTTGTGGTGGCATAGC & \\
\hline M-salm-R & CCTGAGCCTGGGCTACC & \multirow{2}{*}{ salm, Musca } \\
\hline M-salm-F & TCAGCCACCAGCTGTTATG & \\
\hline Md-dpp-3'-F & GAAGGTACCAAAGGCATGCTG & \multirow{4}{*}{ 3'-UTR, Musca } \\
\hline Md-dpp-3'-F1 & TTCAAGTTGCTAGCACCGAGG & \\
\hline Md-dpp-3'-R & CССТСTCССТAAAAGTATGCC & \\
\hline Md-dpp-3'-R1 & AACCAAAAAGCTGGCTGCAGG & \\
\hline
\end{tabular}

Table 11.4: Primers used to amplify gene fragments for ISH probes. Primer names, sequences, and gene name are shown. Bold letters show the T7 RNA Polymerase site.

\begin{tabular}{|l|l|l|}
\hline Name & Sequence 5'->3' & Vector \\
\hline pJet-F-T7 & TAATACGACTCACTATAGGCGACTCACTATAGGGAGAGCGGC & \multirow{2}{*}{ pJET1.2/blunt } \\
\cline { 1 - 2 } pJet-R-T7 & TAATACGACTCACTATAGGAAGAACATCGATTTTCCATGGCAG & \\
\hline M13_F & GTAAAACGACGGCCAGTG & \multirow{2}{*}{ pCRII } \\
\hline M13_R & CAGGAAACAGCTATGAC & \\
\hline
\end{tabular}


Table 11.5: Primers used to design CRISPR/Cas9 constructs. Primer names, sequences, and the purpose are shown.

\begin{tabular}{|c|c|c|}
\hline Name & Sequence $5^{\prime}->3^{\prime}$ & Purpose \\
\hline pJet-F & CGACTCACTATAGGGAGAGCGGC & \multirow{2}{*}{$\begin{array}{c}\text { colony PCR } \\
\text { (pJET1.2/blunt vector) }\end{array}$} \\
\hline pJet-R & AAGAACATCGATTTTCCATGGCAG & \\
\hline M13_F & GTAAAACGACGGCCAGTG & \multirow{2}{*}{ colony PCR (pCRII vector) } \\
\hline M13_R & CAGGAAACAGCTATGAC & \\
\hline attP-F/R & GTAGTGCCCCAACTGGGGTAACCTTTG & $\begin{array}{c}\text { amplification of } \\
\text { attP-3xP3-ECFP-Poly(A)-attP } \\
\text { and ECFP-Poly(A)-attP }\end{array}$ \\
\hline pSL-F & GTTGTAAAACGACGGCCAGTGCCAAG & $\begin{array}{c}\text { confirmation of orientation } \\
\text { in the pSLfa1180fa vector, } \\
\text { sequencing }\end{array}$ \\
\hline ECFP-F & ATGGTGAGCAAGGGCGAGGAGC & $\begin{array}{c}\text { amplification of } \\
\text { ECFP-Poly(A)-attP }\end{array}$ \\
\hline M-HA_L-R & CAGTTCGGGCACTACCCGACAACCACAACCCACCA & \multirow{2}{*}{$\begin{array}{l}\text { amplification of the upstream } \\
\text { homology arm in Musca }\end{array}$} \\
\hline M-HA_L-F & GCCTAATGAGTGAGCCCTACTTAAAATCACCCACCCT & \\
\hline M-HA_R-R & CTGCGCGCGATCGATCCTAGCTGTCAAGTGTGTGG & \multirow{2}{*}{$\begin{array}{c}\text { amplification of the } \\
\text { downstream homology arm } \\
\text { in Musca }\end{array}$} \\
\hline M-HA_R-F & ATTTAAAGCGCTGATTGAGGTTCGAGAGAGGAGAG & \\
\hline
\end{tabular}

Table 11.6: Primers used to design sgRNAs. Primer names, sequences, and species are shown. Blue letters show overhangs for the sticky-end cloning, red letters show GG required for the T7 RNA Polymerase start site, green letters show the T7 RNA Polymerase site, yellow letters show sequences of primers for pSL-T7chiRNA vector.

\begin{tabular}{|c|c|c|}
\hline Name & Sequence $5^{\prime}->3^{\prime}$ & Species \\
\hline Cc-3'dpp-76F & TATAGGCTGTGGGTGTCGATAATA & \multirow{6}{*}{ Ceratitis } \\
\hline Cc-3'dpp-76R & AAACTATTATCGACACCCACAGCC & \\
\hline Cc-3'dpp-77F & TATAGGTCGATAATAAGGACATTA & \\
\hline Cc-3'dpp-77R & AAACTAATGTCCTTATTATCGACC & \\
\hline Cc-3'dpp-78F & TATAGGGGACATTACGGCTATTGA & \\
\hline Cc-3'dpp-78R & AAACTCAATAGCCGTAATGTCCCC & \\
\hline Md-3'dpp-3F & $\begin{array}{l}\text { TAATACGACTCACTATAGATCAGGATATGACAGTGG } \\
\text { TCGAAAGGGGGATGTGCTGCAAGGCGATTAAG }\end{array}$ & \multirow{3}{*}{ Musca } \\
\hline Md-3'dpp-4F & $\begin{array}{l}\text { TAATACGACTCACTATAGATATGACAGTGGTGGGTT } \\
\text { GCGAAAGGGGGATGTGCTGCAAGGCGATTAAG }\end{array}$ & \\
\hline Md-3'dpp-5F & $\begin{array}{l}\text { TAATACGACTCACTATAGAGTGGTGGGTTGTGGTTG } \\
\text { TCGAAAGGGGGATGTGCTGCAAGGCGATTAAG }\end{array}$ & \\
\hline MK152 & AAAAGCACCGACTCGGTGCCAC & Universal primer \\
\hline
\end{tabular}




\subsection{Identification of Ceratitis and Musca genes of the wing development}

Table 11.7: Results of BLASTP search in C. capitata and M. domestica with D. melanogaster proteins.

\begin{tabular}{|c|c|c|c|c|}
\hline $\begin{array}{c}\text { Drosophila } \\
\text { protein }\end{array}$ & Found protein & $\begin{array}{l}\text { Query } \\
\text { cover }\end{array}$ & Identity & $\begin{array}{c}\text { Accession \& } \\
\text { transcriptom reads }\end{array}$ \\
\hline \multirow{3}{*}{ Dpp } & $\begin{array}{l}\text { PREDICTED: protein decapentaplegic isoform } \\
\mathrm{X} 1 \text { [Ceratitis capitata] }\end{array}$ & $71 \%$ & $74 \%$ & XP_004531487.2 \\
\hline & $\begin{array}{l}\text { PREDICTED: protein decapentaplegic isoform } \\
\mathrm{X} 2 \text { [Ceratitis capitata] }\end{array}$ & $71 \%$ & $74 \%$ & XP_004531486.1 \\
\hline & $\begin{array}{l}\text { PREDICTED: protein decapentaplegic } \\
\text { [Musca domestica] }\end{array}$ & $75 \%$ & $79 \%$ & $\begin{array}{c}\text { XP_005178144.1 } \\
\text { comp65369_c2_seq5 }\end{array}$ \\
\hline \multirow{2}{*}{ En } & $\begin{array}{l}\text { PREDICTED: segmentation polarity homeobox } \\
\text { protein engrailed-like [Ceratitis capitata] }\end{array}$ & $35 \%$ & $88 \%$ & XP_012154673.1 \\
\hline & $\begin{array}{l}\text { PREDICTED: segmentation polarity homeobox } \\
\text { protein engrailed [Musca domestica] }\end{array}$ & $100 \%$ & $99 \%$ & $\begin{array}{c}\text { XP_011293671.1 } \\
\text { comp65073_c0_seq1 }\end{array}$ \\
\hline \multirow{3}{*}{$\mathrm{Hh}$} & $\begin{array}{l}\text { PREDICTED: protein hedgehog } \\
\text { [Ceratitis capitata] }\end{array}$ & $84 \%$ & $64 \%$ & XP_004533713.1 \\
\hline & $\begin{array}{l}\text { PREDICTED: protein hedgehog isoform X1 } \\
\text { [Musca domestica] }\end{array}$ & $84 \%$ & $62 \%$ & XP_005179451.0 \\
\hline & $\begin{array}{l}\text { PREDICTED: protein hedgehog isoform X2 } \\
\text { [Musca domestica] }\end{array}$ & $84 \%$ & $62 \%$ & $\begin{array}{c}\text { XP_005179451.1 } \\
\text { comp63797_c0_seq1 }\end{array}$ \\
\hline \multirow{3}{*}{ Ap } & $\begin{array}{l}\text { PREDICTED: protein apterous isoform X1 } \\
\text { [Ceratitis capitata] }\end{array}$ & $100 \%$ & $79 \%$ & XP_004526276.1 \\
\hline & $\begin{array}{l}\text { PREDICTED: protein apterous isoform X2 } \\
\text { [Ceratitis capitata] }\end{array}$ & $52 \%$ & $82 \%$ & XP_012157689.1 \\
\hline & $\begin{array}{l}\text { PREDICTED: protein apterous } \\
{[\text { Musca domestica }]}\end{array}$ & $100 \%$ & $74 \%$ & XP_011296384.1 \\
\hline \multirow{2}{*}{$\mathrm{Wg}$} & $\begin{array}{l}\text { PREDICTED: protein wingless } \\
\text { [Ceratitis capitata] }\end{array}$ & $100 \%$ & $74 \%$ & XP_004519434.1 \\
\hline & $\begin{array}{l}\text { PREDICTED: protein wingless } \\
{[\text { Musca domestica }]}\end{array}$ & $73 \%$ & $78 \%$ & $\begin{array}{c}\text { XP_005187529.1 } \\
\text { comp65904_c1_seq1 }\end{array}$ \\
\hline \multirow{7}{*}{$\mathrm{Omb}$} & $\begin{array}{l}\text { PREDICTED: optomotor-blind protein-like v.1 } \\
\text { [Ceratitis capitata] }\end{array}$ & -- & -- & XP_004530554.1 \\
\hline & $\begin{array}{l}\text { PREDICTED: optomotor-blind protein-like v. } 2 \\
\text { [Ceratitis capitata] }\end{array}$ & -- & -- & XP_004530463.1 \\
\hline & $\begin{array}{l}\text { PREDICTED: optomotor-blind protein isoform } \\
\mathrm{X} 1 \text { [Ceratitis capitata] }\end{array}$ & $67 \%$ & $84 \%$ & XP_012159217.1 \\
\hline & $\begin{array}{l}\text { PREDICTED: optomotor-blind protein isoform } \\
\mathrm{X} 2 \text { [Ceratitis capitata] }\end{array}$ & $67 \%$ & $83 \%$ & XP_012159218.1 \\
\hline & $\begin{array}{l}\text { PREDICTED: optomotor-blind protein isoform } \\
\mathrm{X} 3 \text { [Ceratitis capitata] }\end{array}$ & $67 \%$ & $83 \%$ & XP_012159219.1 \\
\hline & $\begin{array}{l}\text { PREDICTED: optomotor-blind protein [Musca } \\
\text { domestica] v.1 }\end{array}$ & $48 \%$ & $78 \%$ & $\begin{array}{c}\text { XP_005177796.1 } \\
\text { comp59712_c2_seq1 }\end{array}$ \\
\hline & $\begin{array}{l}\text { PREDICTED: optomotor-blind protein-like } \\
{[\text { Musca domestica] v.2 }}\end{array}$ & -- & -- & $\begin{array}{c}\text { XP_011295211.1 } \\
\text { comp59712_c3_seq1 }\end{array}$ \\
\hline
\end{tabular}




\begin{tabular}{|c|c|c|c|c|}
\hline $\begin{array}{l}\text { Drosophila } \\
\text { protein }\end{array}$ & Found gene & $\begin{array}{l}\text { Query } \\
\text { cover }\end{array}$ & Identity & $\begin{array}{c}\text { Accession \& } \\
\text { transcriptom reads }\end{array}$ \\
\hline \multirow{6}{*}{ Spalt } & $\begin{array}{l}\text { PREDICTED: homeotic protein spalt-major } \\
\text { [Ceratitis capitata] }\end{array}$ & $54 \%$ & $34 \%$ & XP_012159662.1 \\
\hline & $\begin{array}{l}\text { PREDICTED: homeotic protein spalt-major } \\
\text { isoform X1 [Ceratitis capitata] }\end{array}$ & $14 \%$ & $90 \%$ & XP_012159663.1 \\
\hline & $\begin{array}{l}\text { PREDICTED: homeotic protein spalt-major } \\
\text { isoform X2 [Ceratitis capitata] }\end{array}$ & $13 \%$ & $93 \%$ & XP_012159664.1 \\
\hline & $\begin{array}{l}\text { PREDICTED: homeotic protein spalt-major } \\
\text { isoform X3 [Ceratitis capitata] }\end{array}$ & $14 \%$ & $76 \%$ & XP_012159665.1 \\
\hline & $\begin{array}{l}\text { PREDICTED: homeotic protein spalt-major } \\
\text { isoform X1 [Musca domestica] }\end{array}$ & $99 \%$ & $57 \%$ & $\begin{array}{c}\text { XP_005178074.1 } \\
\text { comp67087_c0_seq2 }\end{array}$ \\
\hline & $\begin{array}{l}\text { PREDICTED: homeotic protein spalt-major } \\
\text { isoform X2 [Musca domestica] }\end{array}$ & $99 \%$ & $57 \%$ & $\begin{array}{c}\text { XP_011290281.1 } \\
\text { comp67087_c0_seq2 }\end{array}$ \\
\hline \multirow{3}{*}{ Kni } & $\begin{array}{l}\text { PREDICTED: zygotic gap protein knirps isoform } \\
\text { X1 [Ceratitis capitata] }\end{array}$ & $99 \%$ & $36 \%$ & XP_004522172.1 \\
\hline & $\begin{array}{l}\text { PREDICTED: zygotic gap protein knirps isoform } \\
\text { X2 [Ceratitis capitata] }\end{array}$ & $99 \%$ & $35 \%$ & XP_004522173.1 \\
\hline & $\begin{array}{l}\text { PREDICTED: zygotic gap protein knirps } \\
\text { [Musca domestica] }\end{array}$ & $100 \%$ & $51 \%$ & $\begin{array}{c}\text { XP_005186819.1 } \\
\text { comp64709_c0_seq1 }\end{array}$ \\
\hline \multirow{12}{*}{ Dad } & $\begin{array}{l}\text { PREDICTED: mothers against decapentaplegic } \\
\text { homolog } 7 \text { isoform X1 } \\
\text { [Ceratitis capitata] }\end{array}$ & $99 \%$ & $34 \%$ & XP_004524253.1 \\
\hline & $\begin{array}{l}\text { PREDICTED: mothers against decapentaplegic } \\
\text { homolog } 7 \text { isoform X2 [Ceratitis capitata] }\end{array}$ & $99 \%$ & $33 \%$ & XP_012156609.1 \\
\hline & $\begin{array}{l}\text { PREDICTED: protein mothers against dpp } \\
\text { isoform X1 [Ceratitis capitata] }\end{array}$ & $50 \%$ & $28 \%$ & XP_004530256.1 \\
\hline & $\begin{array}{l}\text { PREDICTED: protein mothers against dpp } \\
\text { isoform X2 [Ceratitis capitata] }\end{array}$ & $50 \%$ & $29 \%$ & XP_004530257.1 \\
\hline & $\begin{array}{l}\text { PREDICTED: mothers against decapentaplegic } \\
\text { homolog } 3 \text { [Ceratitis capitata] }\end{array}$ & $29 \%$ & $34 \%$ & XP_004523592.1 \\
\hline & $\begin{array}{l}\text { PREDICTED: mothers against decapentaplegic } \\
\text { homolog } 4 \text { isoform X1 [Ceratitis capitata] }\end{array}$ & $29 \%$ & $30 \%$ & XP_004519586.1 \\
\hline & $\begin{array}{l}\text { PREDICTED: mothers against decapentaplegic } \\
\text { homolog } 4 \text { isoform X2 [Ceratitis capitata] }\end{array}$ & $29 \%$ & $31 \%$ & XP_004519587.1 \\
\hline & $\begin{array}{l}\text { PREDICTED: protein mothers against dpp } \\
\text { isoform X1 [Musca domestica] }\end{array}$ & $29 \%$ & $34 \%$ & $\begin{array}{c}\text { XP_005175100.1 } \\
\text { comp59662_c0_seq1 }\end{array}$ \\
\hline & $\begin{array}{l}\text { PREDICTED: protein mothers against dpp } \\
\text { isoform X2 [Musca domestica] }\end{array}$ & $29 \%$ & $34 \%$ & $\begin{array}{c}\text { XP_005175101.1 } \\
\text { comp59662_c0_seq1 }\end{array}$ \\
\hline & $\begin{array}{l}\text { PREDICTED: mothers against decapentaplegic } \\
\text { homolog } 3 \text { [Musca domestica] }\end{array}$ & $29 \%$ & $34 \%$ & $\begin{array}{c}\text { XP_005185553.1 } \\
\text { comp61208_c0_seq1 }\end{array}$ \\
\hline & $\begin{array}{l}\text { PREDICTED: mothers against decapentaplegic } \\
\text { homolog } 4 \text { [Musca domestica] }\end{array}$ & $29 \%$ & $31 \%$ & $\begin{array}{c}\text { XP_005191545.1 } \\
\text { comp66267_c1_seq1 } \\
\text { comp66267_c1_seq3 }\end{array}$ \\
\hline & $\begin{array}{l}\text { PREDICTED: mothers against decapentaplegic } \\
\text { homolog } 6 \text { [Musca domestica] }\end{array}$ & $82 \%$ & $40 \%$ & XP_005182029.1 \\
\hline
\end{tabular}


List 11.1: RACE and CDS fragments amplified on C. capitata and M. domestica embryonic cDNA with the gene specific primers. Sequences of the primers are shown in bold.

$>$ Cc-ap-5'-UTR (739bp) in PCR ${ }^{\circledR} I$

CGAGATTACGATTTTTTTTTTTTTTTGTAAATAAGCTAAAGTTTATACTTAATTAAATTGA TCTTAAACGGAGTGAGTGAAAAAAATTGAATGTGCTAACTAATCATGACAAAAAAAAAAAACAT TATTTAGTTGCGACTAAATAAAACAAAAGATCGTGCGCGATATAAGTAGAACTACTACTAAAAG CTTAAATATATATCTTGGAACATCGGTCCTCCTGGCTTTTGGGTGGCTCCTTCATCCACACAAAA TGGTTACGCAGCGAATTCTACCTACGATATGAAAGGAAATATGTCCGTAGCTTCTGCATCGAAA AATATACAGCAGAGCGCAAGATTTCTTGGGCCAGGTGCAAGAGAAAAAAGTCCAACTCCACCT GTGGCACATCAAGGAAGCACTCATTGCGGCAGTGCTGCAGGTGTAAATAACAATCGTTTACTTT ATCGATCCTGCTCTTCCTGTCCAGATCTTTGTGACCACAATACAAAGCCTTACAGCTCGACGTTT AGTGAGCCATTTAAAAGCTATGAGACAGCAGACCGTGCATTATTCAGCGAAACTGCTCTCAAAT ATGCGATAAGCCGAGCGCGGGCTGACTGTTTAGAAGTGAGTGAGGAAACTACCTCGAGGATAT CCTTTAAAACGGAACCATTCGGTCCACCAAGTAGCCCGGACTCCACAAATGAAACACAAAATCT GCCTCGAAGCATAGAAGATTGCGCTGGTTGCGGTCGGT

$>$ Cc-ap-5'-UTR (151bp) in pCR ${ }^{\oplus} \mathrm{II}$

ACGCGGGCTGACTGTTTAGAAGTGAGTGAGGAAACTACCTCGAGGATATCCTTTAAGAC GGAACCATTCGGTCCACCAAGTAGCCCGGACTCCACAAATGAAACACAAAATCTGCCTCGAAGC ATAGAAGATTGCGCTGGTTGCGGTCGGT

$>$ Cc-ap-3'-UTR (889bp) in pCR ${ }^{\circledR} I$

GGTGGCGGTAGCGGTTCCCCTCAACATACACTCGAGTAGCAGTGGTGTTCGCTGCAGTT ATCGCACAGCGAGCTTTGAGCTAAAATAGATAATAACATACATACATTTGTACATATATATTGC TGCTTTATAAATGTAACAGCCGTAGTTGTAGCTGTAGTTTAAAGCACTAGCTTTTCCGCAAGCG CACCAGTCCAATGTAAAGGAGAAATCACGGCAGGGAATTCACCGCCTACACACGCACTCATGC GTTTATACACTTCCACACAATACAATCATACATATAATTCATATGTATGTATGTTAAATAACAAA CGCTCATGTCAGCATAAATCTGCAAGTAGATACATATTTACATACATATGTACATATGTATATAA ATACAATCCACGGCAATACACAAAATACTATATAACTCATTGGAATTGTATTTATTAAGTTTTGC AGCGAATGTATCCAGAAATTCAATATCGCATAGTCTTATAATTGAATTCAAATACGTACACGAG CAAATTAGTTTAAACCAATACCGGAACATTAGTGAACATACCCTGCTAAACAAGCTGTAAGCGT GCACTGACTAAGTGTGGTTATTAAAATTTTCGACCTTATAAGAAATAGGCAATGTTTCGATTTTC CGATTATCAGAATCATGCTTAAAATTTTGAATATGAACGCCTCATCGCACGCCGAATGCCCGAC AGTCTCATTGAGGTTCACTGATGGTCATGTCAGCAACAGAATTTTTTTTAAAATAAGATGGATG AAGAAAAATTCGTTAAAAATCTTAGAAATCGGCGGCTGTATCTCAATTAACCGATTTGTCTGAT ATGAGGTTTTGGGTAAAAATAAAACGCCGGAATACAAAAAAAAAAAAAAAAAAAAAAAA 
>Cc-dpp-5'-UTR (273bp) in pCR ${ }^{\circ} \mathrm{II}$

CACTTGGCATTTGCTACTCAACGGTTTAGTTAGTAAACGTGTTCGCTCGTACCAAATCGG CTACTCGATTGCTAGATTACTATAAAAATATATATAGACTATAAATACATACATACATATATATG TGTATATTGTGCATTGTGGTGTCGCTGTAGTACGTCGTCGATTGTTTTTAACCCTTTGACTAACG TTGAGCAATTTCCATGGTTTTGTATCGTTTCAGAGTTGAAAGCGACCATGCGCGCATGGCTTCT ACTCCTCGCAGTGCTGGCG

$>$ Cc-dpp-5'-UTR (61bp) in pCR ${ }^{\circ} I$ TATCGTTTCAGAGTTGAAAGCGACCATGCGCGCATGGCTTCTACTCCTCGCAGTGCTGG CG

$>$ Cc-dpp-3'-UTR (936bp) in pCR II

AAGTGCCAAAGGCCTGCTGTGTGCCCACCCAATTGGAGGGTATCTCTATGCTCTATCTG AATGACCAGAATACCGTAGTGCTGAAGAACTATCAGGATATGACAGTTGTGGGCTGTGGGTGTC GATAATAAGGACATTACGGCTATTGACGGAGCAGCAGTAGCAGCAGCAGCAGTAAGAGTTAAG AAAGAGTGAGTAAGAACACAAAGAAAAAATGCTCGTGCTCATGACGATAAAAGCCACAGAGTA GCGCCGAAAAACTGCGTTGATATTCGGTAAAACGAACTAGAAAACGAATACATATTGAACCAG AAGCCAAACCAATGTCGTCGATGGTATATCTAAAGTGTACATCGACCACAAAATTGTGTGTTTA TTACGACGTGTGCAAGCGCTCGACCTATGCTAACGCAACGAAGAGCCAAAGCAAATTCAATAAG CCGCTGTTGCAGCGATCTAAAGAGTACACAGCGAGAGCACGAGAACACATGACGCTATAGGTG AGCGTAAATTAACAACCGCTAGCCGTACTACTCTTGCCGACCAAAAAGGACAAAAGTCCACCTA AATAGAGAAGTTTCGTACCCACACGCACACGCACCCACGGCGTAGACGCCGAGCACATGCACA TCGGGAGCGCAGACAAGAGAGCATTAAATGCTAGGTTTCAAGCCGCTTCACGCTTTAATAAGTT TAAATTTTTATTAAACGAAATGCAAACAAAATGGACTGAATATATGAATTTTAAGTATAAATTTT AGACTTTATTTTTATTAAATAGAGCAAGTAGAAAAACGAAGCTGAGTTGCGTTGAGAGTTAATG GAACTGAGAGTTGCGCCACGCGATCGTCGCAATTGACAGCCAGGCTGCCAAGACGTTTGACAAT CACGGCCGACACAATGAACAAAAAAAAAAAAAAAAAAAAAAAAAAAAAA

>Cc-en-5'-UTR (151bp) in pCR ${ }^{\oplus} \mathrm{II}$

GTGCCAGGTTATTGATTAGTTTGTGCCCCTTACGTGTATACTCAACGTGTGTCCTTAACGC GTTCGTATATAAAATTGCCACGGCACTACCAATGGCCCTAGAGGATCGCTGCAGTCCGCAATCT GCTCCAAGTCCGCCTGGCCTACCGCA

$>$ Cc-en-3'-UTR (578bp) in pCR II

CCGCGTACCGCCTTCTCCAGCGAACAGTTGGCGCGTTTGAAGCGCGAATTCAATGAGAA TCGTTATCTTACCGAACGTCGTCGTCAACAGCTGAGCGCTGAATTGGGTTTAAATGAGGCGCAA ATCAAAATTTGGTTCCAAAATAAACGCGCAAAAATAAAGAAATCTTCAGGTTCCAAAAATCCGC TCGCTCTCCAACTGATGGCACAAGGACTCTACAATCATACGACAGTGCCGTTGACGAAAGAGGA 
GGAGGAGCTTGAAATGCGTATGAATGGACAGATCCCATAAACGGACGGGTGGCGCTAGAGTTG CTTGTGGGAGGGGAATAAGAAACGAGTAGAAATAAATATATGTATTCGTTGCCTCTCAACTGCG GGTAATCTAAATTTGACGACGCGTGCAATAATTAGGGTAGGATAGGGAAGAAACAACTGAGTTT ACGGTCACCAGCCGTCGGTAGAGTTGATGAGAAGATATAAAATATATGTTCTTAAAAGCAAAGT AAAAATTTGTTATGTGATATAAATATATATACGTAAGTATGTATGGCAAAAAAAAAAAAAAAAA AAAAAAAA

$>$ Cc-hh-3'-UTR (25bp) in pCR ${ }^{\oplus} I$

CGATATCCGCTGCGACGACGCCCACAGCAGGTATACACTGGTATGCGCGATTTTTATAT GCAATAAAGGATATTGTGTTGCCGCAGGAGTGGCGTTACCAGTGACCTGGCGGAGGAGAGCCG CACAGCTGCTAGTTGGCAGGCCTCGTTCGCTATTTAGCCTATAAAGTACCAAGCTCGGTGCAAA ATGCTGATTTAGGCAAAATGAGACATCACCGTTTAGTTTTTAGAAAAAAAAAAAAAAAAAAAAA AAAAA

$>$ Cc-kni-5'-UTR (850bp) in pCR II

AGTTCACTGCCAGTTCTCGAAGCGTAGAAAACGTAAAACGCGCGAATCAAGAGAAAAAT TTAAAAGCACACAACATACAAGTGGCTTAATATACATACATACATATCTATATCAGTGTGGTAA ACAACTTTGAAGACTAGTTTTCTTACACGACGTTTTTGGCTAAGTACTAAGCGGAAAAACAAAA TGAGAATTACGTTGTAAAGTAAAAAAAACAATACAATATAAAAAAGCTTATTCAGTAACTTTAA GAAAACTTTTTTGGAATTTATCGCAAACGCAAGTGTGACAATTACATTGGCTGCATGAGACGAA ACAAACATATTTTAATATTAAATATAATTGTCAAACAACTGTTTATTATAAACCAATAAAGTGCA GAAAATTCTAATTGGCGCTGTTTGAGTAGAAAAAGAAAATCAAAACAATGAACTGAAAAACCCA GCTGTTAGATTGGAAACAATAATATTGCGCAAAGTGTTAATAAAAAATAAATATAAACTTATAC AAAAAACAATATCTGCTGTTATATACAAAAAATAAAAATAGGCAATAAAAAGAAAACTTCGCTA TACAAGGAGACCAAATAAATAGTCAATCAAGATGAATCAAACGTGCAAAGTATGTGGTGAACC AGCAGCTGGTTTTCATTTCGGTGCTTTTACATGCGAGGGCTGTAAGTCCTTTTTCGGCCGCTCCT ATAACAATCTCTCCTCGATCACTGAGTGCAAAAACAATGGCAAATGCGTTATTGACAAGAAGAA TCGCACTACCTGTAAGGCTTGCCGCCTACGCAAATGCTATGCCGTGGGCATGTCAAAGGGTGGT TCGCGCTATGGCCGTCGTTCCA

$>$ Cc-kni-3'-UTR (348bp) in pCR II

CAGCATCAACGGGGTCGgCGGTGTCTAGCAAGCGCGGTATATATATGTGTGCGTAAACA CAGCGCATCTAGCATAACCACCACACATCCACTCGGTACTTGGAAAGGCCGAAGAGAAAAGCT GACGCGGTAGGAGGAGCTCGCTCAATGGTCTGAGCCTCAAACGCCGGCATACGGGCTGTTAGT CTCTACTGATGTTAAGTAGTTTTGTTGTAGTTTATCATCCAACGTGCATGTGGAATAACGGAATG CAAATGAGTGCCGCCAAATACATGCTCACGCGTAGCGTTACAAAGGCAATTGCAATAGTTATTG TTGTACAATACATTCGCGCCGCGCACTCTGCGTT 
>Cc-mad-5'-UTR (433bp) in pCR ${ }^{\circledR} \mathrm{II}$

TCGTGCAATAGAATTATTGACATTTGCATATAAGCATTTCTTTGAGGAAAGTGAAAACGT TTATTGTTTTTAATTCTGCAATATAACATTTTCACGTGCAGTGAAGTACATGTACACTTAGCTAT ATTTGAAGTATCTATGTATCTATGGTTTTCTTTGAGGTCGTACTCGTTGTCCATCGTTCGTCATA TTCGTTTGTCGTGTCGAGTTCGTGTCCGTTTGTCGCTGCATTTCCTGATAAACAATAGAAATTTG TGAAATTAATGAAGTCGCAGTGCGATTCCGTAGAAAAACTTGTTAAATAAGAACAGTAATTGCC GTTTATCAATCAAATTATCATCCACTTAACAGTTATCCCGGTTATCAGATGAACCCGACAAACG GCATGGATACAGAAGATGTCGAATCCACTTCCAGCAGCGCCATGTCCAGA

$>$ Cc-mad-5'-UTR (188bp) in pCR ${ }^{\circledR} \mathrm{II}$

ATGAGTTGACAGTAGTTAACATAAATGTATATTTTATGCTATAGAATTTAATTGTTTTTTT ACTTCCAGTATTTATAAAGAATAAAGATTATCATCCACTTAACAGTTATCCCGGTTATCAGATGA ACCCGACAAACGGCATGGATACAGAAGATGTCGAATCCACTTCCAGCAGCGCCATGTCCAGA $>$ Cc-mad-3'-UTR (250bp) in pCR ${ }^{\circledR} \mathrm{II}$

TGGGCTCGCCGCATAACGCCATCAGTTCCGTATCATAAAAGCAAAATGATATTGGTACA TACAGGCATATTTCATTCTATTTACATTTCACCATCTACAAGAGAAAAAATCAACAATATGAAAT GAACTTGTAGCAATTTCTTTGACAAAAAATAAAGAAAATTTGGATAATTCATATTTGAATTAATA AAGGTATACGTATTGTAAATTTTCACCTGATCTAAAAAAAAAAAAAAAAAAAAAAAAAAAA

$>$ Cc-mad-3like-5'-UTR (384bp) in PCR ${ }^{\circ}$ II

CAGGCACACACAGACATATTATTCCGGTATAAGTAATAAACAAGAGGATTAATTCAATCA GCTCGCGGGGAAAGGCTGATTACTACAGTAAACCACTTTATTAATATCCACTTGCCACTAAAAA GTATTTGGCGAAAGAAAAAAATATAATATTAAAACTTTTGTGTGGGTAAAGGTAAATAACAACA CGCTTATCTTGAACTGCTTGAAGCGAGCGTTAAATTAGCATTGCGCTAAATCGGTAATATTTGTT GACGCACACTTGTCGACCAGTGCACTCTTCGCGTGCCACGTGCGATGTTGCCATTCACCCCACA AGTAGTTAAACGTTTGTTAGCACTCAAGAAGGGCAACGAAGACACGGTCGAGGGCAAGTGGTC CGAG

>Cc-mad-3like-5'-UTR (321bp) in pCR ${ }^{\oplus I}$

CGCGGGGAAAGGCTGATTACTACAGTAAACCACTTTATTAATATCCACTTGCCACTAAAA AGTATTTGGCGAAAGAAAAAAATATAATATTAAAACTTTTGTGTGGGTAAAGGTAAATAACAAC ACGCTTATCTTGAACTGCTTGAAGCGAGCGTTAAATTAGCATTGCGCTAAATCGGTAATATTTG TTGACGCACACTTGTCGACCAGTGCACTCTTCGCGTGCCACGTGCGATGTTGCCATTCACCCCA CAAGTAGTTAAACGTTTGTTAGCACTCAAGAAGGGCAACGAAGACACGGTCGAGGGCAAGTGG TCCGAG 
$>$ Cc- mad-3like-3'-UTR (624bp) in pCR ${ }^{\oplus} \mathrm{II}$

TGGTTGATGAAAAGTAGCGAGGCGCAGGAAAAATTTTTAATTTTATAAGAATTTCGACA GTGAACGCAAAAGAAAAGCTCCATAATGATTATGAAAATTAGAAACATTTTTTGGGCTTTCTAC AGTTCGTTGACCACATGCAAAAATGTATGTATACATGTCGAAGCAAAAACCATAGACAAATTAA TTAATTATGCTGTAGTCGAAACACATTATAAAATCTATTTACGTGTCTTCTATTTTTACAAACAA CTTAAAGGACACTCTCAAGCAAAGCGTATTAATAGTAAATGGGCTAGACTGCCTTTGGCATACA AATTTATATAAGAAAGTATTACAATTCACATTAAGCCAGGAATTGTAAGCTGTTTCGCTGTTTCT TATCCTTTTTGAACTAATCGCCGCAATACAATGCTTGTATTTAATGGAATAATGAGAAGGCATAT CGGTCTGGCGCACTTTATTATTAAATCGTTTTGTTCACAAAGTAGTACACTCTCCTTATAGATTC ATGTGTATAAATGTATGCATAAACATATCCATACATACATATGCAATAAAAATAAAAATAATCT CAAGTAGCAATATACTTGAAAAGAAAAAAAAAAAAAAAAAAAAAAAAAA

>Cc-mad-4like-5'-UTR (757bp) in pCR ${ }^{\oplus} \mathrm{II}$

GCGGGGACGGACGAACTGCGACACTACCGAGAATGAACAAAGAAATTTGTTTGGAATCG CGGAAAAGTTGAAAAGCGAGAAAAATAATTAATATATAAAGTTGCAAAAATAAAGTAAGCCGC AAGGCGGGCGTTGTTAACGTTTGACCACAAGCTGTGCGGCGTTCAATTGTGCGTAAAAATAGAA TTGCGGTATATGAATGTGTGCGTAAAGAAAAGTAAAATATAAAAGAATACCAAAGGAGGTGAA AAAATTTTCTGCTATGATCGGTTGTGGATGGAGCTACTAAGTAAAACTATTTACAAAAAATTAA ATAAGAATTCATAATTTAATTAAACAGCGAGTGATAAAAGCAAAAGTTTTTCGGAGTTACATAT TTTGTTCGTTTTTAATAAAATTAACACACAAACTGAGTTTATACATCGTCCACCCAAGGTGGATA ATGAAGGACAAACAGTGTACGGGAAGCGTTTATTTGTATTATTTTATAATAATCAAGAGAGCTT AAGGAACTATTTCGATTAAGGTCTACTTTTGGTTTATTATATTACGCTGAAGTTCTTGCAGCACT TATTTTCTTTTTGAATTGGTTGGCGGCTTAGTATCCGCCTAAAAATATCCGCCAATTTGTGGTCG TCATCCGGATTTGCTTTTTGTGACGGCGACAGCCCGAACGCCGGCAAAGTGGATGCTCGGGGAA GTCTGGGGGTTCCCCAGGACTATACAGGCATGGTGGGCTTGCCAGGTGGTGGTGGTG

$>$ Cc-mad-4like-5'-UTR (297bp) in PCR ${ }^{\circledR} I$

ACGGGAAGCGTTTATTTGTATTATTTTATAATAATCAAGAGAGCTTAAGGAACTATTTCG ATTAAGGTCTACTTTTGGTTTATTATATTACGCTGAAGTTCTTGCAGCACTTATTTTCTTTTTGAA TTGGTTGGCGGCTTAGTATCCGCCTAAAAATATCCGCCAATTTGTGGTCGTCATCCGGATTTGCT TTTTGTGACGGCGACAGCCCGAACGCCGGCAAAGTGGATGCTCGGGGAAGTCTGGGGGTTCCC CAGGACTATACAGGCATGGTGGGCTTGCCAGGTGGTGGTGGTG

$>$ Cc-mad-4like-3'-UTR (2061bp) in PCR ${ }^{\circledR}$ II

CGGCTGGTCCGTTAGTAGGCGGGATGGATATTGATGGCAACGACATCGGAACTATACAG CATCATCCTTCACAGTTAGTTGGTCCGGGCTATGGATATCCACAGGCGGCAGCTGTACCGCATG GTATGTCACTGACTGGACCCATGAACCCCGGTGGCCCAGTAATGGGTCCACCTCCCCCGCCACA 
AATGCAGACTCAACAGGGCAACGCTCCGAATGGCAACATTTTAGCTGGAGCTGGTCATCACTCT CAAACGAGTTCTCCAAATGATGGATTACAACAGACCCAGAATTCGGCAGGTGCCGGCGGCAAT GCAGTCGGTGTCGGAGTCAACGTTAGTGGTGGATCAGGTGCACCAGGATCAAATCAAGGCACC TACTATGGGCCACCAACAACATCACAAATGCCCAATGTAGCAGAGGCCAAATTCAATGCTGCAT TGAATAGTGCCGGGCCGGGTGGTGGCTTACAGCAACCGCATTCTCCACATCTACAACAGAATGG TTATGTAACAGGTGGCGGTGCGCCTTCAAACTATGGACAACAAGTAACTAGTGGCGGTAATGCT GGTGGTAGCAGTGTGCAATCCGCTGTACAAGCAAATTCAGGTGGACAAGGAGTGTCTGGTGGT ACAGTAGGTGCCGGCGGTACTTGGACAGGATCCAGCACGTTAACCTACACGCAATCCATGCAAC CCCCCGATCCACGCACTCATCCCACTGGTTATTGGAATGCCCCATTGAGTGGCGATATAAGTGG AAATCAGCAACAACAACAACAACCACGCATGTTGTCCCGCCAGCCAGCACCAGAATATTGGTGT TCCATTGCATATTTTGAATTGGATACACAAGTAGGGGAAACGTTTAAAGTACCATCTGCGAAGC CAAATGTTATTATCGACGGCTATGTTGACCCATCCGGGGGTAATCGCTTTTGTTTGGGAGCTCTT AGTAATGTACATCGTACGGAACAATCAGAGCGTGCTAGGTTGCATATTGGCAAAGGAGTTCAAT TGGACCTACGAGGCGAGGGCGATGTCTGGCTGCGTTGCCTCAGCGACCATTCGGTATTTGTACA AAGTTACTATCTGGATCGTGAAGCAGGTCGTACACCAGGCGATGCTGTACATAAAATTTATCCA GTTGCGTGCATTAAAGTTTTTGATCTGCGTCAGTGCCATCAACAAATGCATTCATTGGCCACAA ATGCACAAGCAGCAGCAGCCGCACAAGCAGCTGCTGTTGCTGGACTACCAAGCTCGCAAATGG GCGGACCACCACGAAATATTACAGCAGCTGCTGGAATCGGTGTTGATGACTTACGTCGCCTGTG TATTTTGCGTTTGAGTTTTGTCAAAGGCTGGGGACCTGATTATCCACGACAATCGATTAAGGAG ACACCATGTTGGATAGAGGTGCACTTGCACCGAGCTCTACAACTGCTGGACGAAGTGCTACACG CAATGCCAATTGATGGTCCGCGGGCGATGGCTTAATGCAAGTTTTTGATTACTCAGATTCATAT ATAAAACTAATGTATGATACGTACACACATTTAAAAAAAAAAGGAGTTCAACACATAATTTAAT TCAGCTCAGTTCATCACAATTAAAGAGAAAGTAAAAAATACTAAATTAGTGGAGTTGGCATATC GAACTTTGTTAAATTAACGCCAGAAGAACACAAAACGATTGAATTTTGTATCAAAAGTAAGAGG GTACGATTATACGACACATACATGACGCATGTTTGTATTTGCAAGATAATCTTCATTATATAAAC CAATTTAAAATCTTATTGATTTTAGTTCGATATTATTTATTTTCTTTTTAATACAGTTAAACAAAT AATGATAAAAGACTCCATAAGTTTTAAAATAATTAATTTTAATATCCTTTTATCGCAATAGTTAA TATTGTCAGCTTTGCCTTTATCTTTTAACATTCTTTGAATGTTTAATATATGTTTAGACCTTTGTG ATTAATCGAAAAAGAAATAAATTACAAATATTGTTTAAATTTTCAATCCGAAAAAAAAAAAAAA AAAAAAAAAAAAAAA

>Cc-mad-4like-3'-UTR (1438bp) in pCR ${ }^{\oplus} \mathrm{II}$

CGGCTGGTCCGTTAGTAGGCGGGATGGATATTGATGGCAACGACATCGGAACTATACAG CATCATCCTTCACAGTTAGTTGGTCCGGGCTATGGATATCCACAGGCGGCAGTTGCCGATTCTA ACCATGTAATGAGTTCTATGTACGTTGCGGGTCGCACAATACCAAAAATTGAGCCTGGCGATGG 
TAGCGGTATGCCTCGTGGTGCTTGGATGGTTCCACCGCCACCACGGTTGGGGCAGTCACAACCA TTGCCACAAACGCAGCCACAACAACATCACAAATGCTGCATTGAATAGTGCCGGGCCGGGTGGT GGCTTACAGCAACCGCATTCTCCACATCTACAACAGAATGGTTATGTAACAGGTGGCGGTGCGC CTTCAAACTATGGACAACAAGTAACTAGTGGCGGTAATGCTGGTGGTAGCAGTGTGCAATCCGC TGTACAAGCAAATTCAGGTGGACAAGGAGTGTCTGGTGGTACAGTAGGTGCCGGCGGTACTTG GACAGGATCCAGCACGTTAACCTACACGCAATCCATGCAACCCCCCGATCCACGCACTCATCCC ACTGGTTATTGGAATGCCCCATTGAGTGGCGATATAAGTGGAAATCAGCAACAACAACAACAAC CACGCATGTTGTCCCGCCAGCCAGCACCAGAATATTGGTGTTCCATTGCATATTTTGAATTGGA TACACAAGTAGGGGAAACGTTTAAAGTACCATCTGCGAAGCCAAATGTTATTATCGACGGCTAT GTTGACCCATCCGGGGGTAATCGCTTTTGTTTGGGAGCTCTTAGTAATGTACATCGTACGGAAC AATCAGAGCGTGCTAGGTTGCATATTGGCAAAGGAGTTCAATTGGACCTACGAGGCGAGGGCG ATGTCTGGCTGCGTTGCCTCAGCGACCATTCGGTATTTGTACAAAGTTACTATCTGGATCGTGA AGCAGGTCGTACACCAGGCGATGCTGTACATAAAATTTATCCAGTTGCGTGCATTAAAGTTTTT GATCTGCGTCAGTGCCATCAACAAATGCATTCATTGGCCACAAATGCACAAGCAGCAGCAGCCG CACAAGCAGCTGCTGTTGCTGGACTACCAAGCTCGCAAATGGGCGGACCACCACGAAATATTAC AGCAGCTGCTGGAATCGGTGTTGATGACTTACGTCGCCTGTGTATTTTGCGTTTGAGTTTTGTCA AAGGCTGGGGACCTGATTATCCACGACAATCGATTAAGGAGACACCATGTTGGATAGAGGTGC ACTTGCACCGAGCTCTACAACTGCTGGACGAAGTGCTACACGCAATGCCAATTGATGGTCCGCG GGCGATGGCTTAATGCAAGTTTTTGATTACTCAGATTCATATATAAAACTAATGTATGATACGTA CACACATAAAAAAAAAAAAAAAAAAAAAAAAAAAAA

$>$ Cc-mad-7like-5'-UTR (798bp) in PCR ${ }^{\oplus} \mathrm{II}$

AGTTTAGTTTCTGCTGTCTATTGCCTGCCGTAAGACATGATTGTGTTTAAGTGATATTGAT TATTTATTTTGCTGACAAAATTCGAGTAAACAAATGCATTGAATTGTGAAAAAGTTTAATGCGA AAAATGCTTATAATATAAGAAATGCGTATAACAAGATGTATGAATTCGGTGTACCATTAGAGTT GATGACTATATATTAATTAACGATTCACATATTTACATAAAGTTGAAGATACGTTATTGATTCAA TCCCAATGAATTAAGTCAAATGCAATAATAAATAAACAACTTTAAAGGAACGACACATATCAAA AATAATTGTTTAAGCAAATAATTTAGTCAAAGCAAACCAGTTTTTAAAAACTTCGATATCGATAA TCTTGCAATAATAATGCTGTTATCATGCATTACATATCATAATTTACAAATTGTCAACAAACCTC GCAATTACAACAGTTTTAACATATCACAAAGGACTTCGCTGAATGATTCTAAAAAATTAATAAA TAAGAGTGCAAAAGATTGATAAATTTGAATTAGTTGATTTTACCCTGGCTATCAGCGAACTTGA TTTAATTTGCATTTTTATTAATTCTGAGCATAAGTATCAAGAGTAGCGAATGTGTTACAAGTTAC AGTTAGTAAATTTTATAATGTTAATATAATAAGTTAAATAAATGTTCCACAAACATTTCATGTCC CTCCATGCTATTTCGAAAGGACAAGGAACTATGGCGTTATGCGTCAAAAAACCTGTCAAGAAAT TTCGATGGGAACAGCAATCTGGAGTCGC 
>Cc-mad-7like-5'-UTR (162bp) in pCR II

GTTACAGTTAGTAAATTTTATAATGTTAATATAATAAGTTAAATAAATGTTCCACAAACAT TTCATGTCCCTCCATGCTATTTCGAAAGGACAAGGAACTATGGCGTTATGCGTCAAAAAACCTG TCAAGAAATTTCGATGGGAACAGCAATCTGGAGTCGC

>Cc-mad-7like-3'-UTR (1943bp) in pCR ${ }^{\circledR} I$

CGACCGCGTTGGCAATCGTTGCTGTGTACATAAACCGTTTGTGAATATTTATGCTGAAG GAAAAAGCTGTAATAATGACAGTCAGGATATCTGTCTACGAAATTTGGCTGCAAATAAAAGTAC ACCATCTCGAGTCGACGTTGAACGCACGCGACAAAAAATTGGTTTAGGTGTAGTATTAAGTCAG GAATTTGAGGGAGTATGGCTTTACAACCGCTCTATGGCCCCTGTTTTCATCCTATCACCAACTTT AAATAAATGTTTGGAGTGCGTCTATAAAGTGGCGCCAGGAGATTGTTTAAAGGCTTTCGATACG AATAGGGCTCAATCGCTTGTTCGTTACACGCAATATCCTACTGCTAAATTAGGCCCGATGAATA TGCATAGCATATGCATTAGTTTCGTCAAAGGATGGGGTAAACATTACACACGGCAAGATATTAT GAAATGTCCATGTTGGCTGGAAATTTCATTTACGCAACGGTGACATCGGAGAACAAATTTAACT GGAGCTGCATGACAAACACTATTACGGCACAGTGCACTATGTGTGAATGGAATTGTTTTTATAA CCAGAAAAATAAAGCTAACAAGGAAAGTTAAGCTTATGCAAAATTTCTGGAGATGCAAACACA GCGGATTAAAACATTTATGTTTTGGGTTTTTAGTAACTGATAAAAGAGGATATATAAAGTTTTGA ATCACATGAATGTACAGAATATTAAAGCAACAACACTCGCTAACAACTTCGATGGCCCATGGTA AAAGCTCATCTTTTAGAATATAATATTTTTATTGTGACTGAGTTAATCGCACATGTTATGTGCAC AATGTGCAATCGCTATTTCCATAAACAAAAATTCTCCATATTTGCAAACGTACATTCCGCCAAA GGATTCTCTGTATTTCATAATAAAATACATGTACATACACGTATATAAAGGACGGCCTGAAAAA GGTTTGATTAAGAGTTTCGATACCAGAATCAAATTGCATTGCCCAAAAAGGTGACCAGGCAAAA TATCGCCAAATTACTATAAATCGATGGTAAATTTTAATATTTTTTGACAAAAATATTACTGTGTC TTTTGCATCTTAGAAATTTTGCGTTAGAAACATTGCAATATTTGTAGGTAATTACTTACAAAGGT ATTGCAAACCTACATACATGTATACAACACGAAGATGAATAAGAGGAGGAAAACCCTGTGATTC AAAAACTAATAATAGCTAAGAATTATTTGAGTTTGTTCTATATTAATAATTTGTATTTTTAAATC AAATAACGTAACCAATTAAAGAGAAAGAAAGCGTTTAACGCTATTTGGTGCCTATATAAAATTG ATATAAACTAGTCTTAAGAACGGATGTGTAACTTACTTTATATGCTTATACATACATACACGTAT GTACATATGTAGTATGTATATGAGAGTGATACAAAAATAAGCTTTTTCCCAAACTGACTAATATT TCCGATTGGATATTAAAAATTATGGTGAACATATGAACCATTAATGTTAATATTAATATAGTACT CATTGCACTTTTCTATTTTATATAGGAATAACTGGTACACTAACCTTCACCTTCTGATATTTATA GTAACATAACCGAGCAACATACAAAAATCATTTAGCTAATATTACTGGTTCAGAAATTACTCGA GTTAAAATTTACCGCAATTTGTATCGCTTGGAATGTACATACTTGTACATAGTAATGACCTTAAT GAAAATAGCTGACTGAAAGCGCTCGAACCGTGATCGCATCAACACACAAAATGCGGTTCTTTTG ATTTTGTTCGACAGCTCATGTATCTTTTGAACCAGCAAACTTAAGGAAAAATCATATCCGATTTT 
TTTTGTATGGTTTAATTTTCTCAAATAATTTGGAATAAATATCGGAAAGTTGTAAAAAAAAAAAA AAAAAAAAAAAAAAAA

$>$ Cc-omb_v.1-5'-UTR (620bp) in pCR ${ }^{\circ} I$

CAAAAACGTCAAAAGAATATGAAATTACAACAAAATCAACAAAAATAAAAAAATCAATT GCTATAACAACAAAAACTAAATGAGAATGCAGTGAAAAATGTGTGTTATAATTCAAATATTGAA ATAATCATTTTCAACATTCATAAAACATTAGTTTTAAAAGCGCCTCAGCTCCGTAAAAGCTAAG GAGAGGTAAACTTTTGTGTCTAATACCCAACGCATGGCTGAAAAACACAGCGATTTAAGCTCAA AATCTCGCTGACGTTGAAAACCATACACTAAAAACCAACTAAGCGCCATTAAACAGCAGTCTCT GCCGGAAAATACTGAACTGCAGGCGCGCCGATAGAGCTGTAATACAAAACCAAGGCGATTTGA AACGCAAAAAATCGAAAACACTGAGGTGTAGACAAAAGCAAATCAAAGCAAAGTGAGATTACA GCTCAGAAATTTGAATTTAGTGAGGTTAAGGCACTTAAGGATAATTGCACGGTCGAAAATTGTA AAATTTTCAGCAGCCGGACTAAAGAAAAAAGTGAGAAAGTAGCAACAGAGTGTTAAAGAGGTG CTCTCAAAGCTATAAGCGGGCAGAAATTGCATGTGTCAGCACTCCGACCGAG

>Cc-omb_v.1-3'-UTR (695bp) in pCR II

CTGTGCCGGGTCATCACGCGTTGGGACCACATCTGCCCCAACAGGCATACTTTCCAGCC GCCGCTCATGCAGCGCTAGCAGGCAGTCCGGCTGGTCCACATCCGGGCTTATACCCAGCTGGTT TGCGTTTCCCGCCACATCATCCGCACGCTCATCTCCCCTTGGGTACACCCTACACAACCGCCGA GGACGTGGTGCTCGCATCAGCGGTGGCGCATCAGCTACATCCAGCAATGAGACCGTTGCGTGC GCTTCAACCAGAAGACGACGGCGTTGTCGACGATCCGAAGGTGACGTTGGAGGGCAAAGATTT GTGGGAGAAATTTCACAAGCTCGGCACGGAGATGGTGATCACCAAAAGTGGACGGCAAATGTT TCCTCAAATGAAATTTCGCGTTTCCGGCTTAGACGCGAAGGCGAAATATATTTTGCTCTTGGAT ATCGTTGCTGCGGACGATTACCGTTATAAGTTTCATAATAGTCGCTGGATGATCGCCGGCAAAG CGGATCCAGAAATGCCAAAACGCATGTACATCCACCCCGATTCGCCGACAACCGGCGAACAGT GGATGCAGAAGGTCGTCTCATTTCACAAATTAAAATTGACCAACAATATTAGTGATAAACATGG ATTTGTAAGTACTGTACGTCTTCTCTGTTTACCGAAAATTTCAAAAAAAAAAAAAAAAAAAAAA

$>$ Cc-omb_v.2-5'-UTR (460bp) in pCR II

AGCGGGAAAAGAATTGTTACAGGCAAGCGCTCATGTCGAATCGCGGGTCCGATTCGGAC AAACTCAATCCTACACATGTGAGCAGCTCACGAGCGCCACTACATTTGGGCCACACAGGACGAC CACCGCATCACATGCTGCCGCATGCGAGCTTACACGACAATCAAGCGGACGATGAGGATAAAC TATTGGATGTGGTCGGACCGCCGCAAAGCCCACTATTGCCTTTGAGTCATTCGTTGCAGCAGAT GCATGCGCATCAGCAATCCGCCCTTGCAGCCTGGTTCAACCACTTGGCCGGCGCTGGTGATCAT GCGAATGAAGAAGCATTACGTCGTCGCCTCCAGTCGGACGATGTGGAACGTGATGGCAGCGAT TCTAGTTGTTCGGAGAGTGTGGGCGGTAGTACGGGCGGCGCTTTCCGACCCACCTCGTCGGGTA GTCCGAAAGAGGGCGCCGC 
>Cc-omb_v.2-3'-UTR (1602bp) in pCR ${ }^{\oplus} \mathrm{II}$

CCTTTGATGCCGTCACGCCCGGCTCAAATGCGAATCGTGTTGGCGGTCCGCCAGGTGCG GGTGATGCCTCTACGCTGGGTCCCGGCAGTAGCGGACCGGGAAGTAGTATAGAAAATGGACCG CGCAGTTTAAGTTCCAGTCCACGACCACGTGCCTCATCTAACTCGCCACCTACACGACCGATTT CCATGTCGCCCACAACGCCACCATCACTGTTGAAGCGTCAAAACTCTCCATCCGAGCTAAAGAG TATCGAGAAAATGGTTAACGGCCTGGAAGTGCATCATAACGGGAGTGGTGCGAGTGGCGGTGT AGGCGGTATGGGTATTGGTATTGGCGCGCTGGTAAGTGGCGCCTTGGAGGAGTTGCAGCGCAA GACGCCAACGCAAATGGATCAGTGACATTTATTTGATCGGGACGATAACGACCGTTCGTCTGTC ATCGATCTCGACGATGTGTGACCGCAAGAGCAAGTTTTCCTCTTGCTTTAGTGGGCTTGCGTAG TAGCCGGAGCAGTGGGCTGGATCCGAAGCCGGCACAGTAGCCTGCATGTATGTGCTGTGCGCG TTGCCTATGAAGCCGAGAAATGTGGAGCAGCCTTATGTGATATGCTGCTTTCGAATATGTCAAT TGTGTGCGAATTCTGCTTCGTCCTCGTGCGCCTGTGGATCGTAAGTCTTTGCTGCAACGCGAAT GTACATTATGTTATCTCGGGTGTGTATATAATAAACCGTTGCAAAAAAACTAGCTTAAGTAGCA TAATAAGCTTCGCAAATGATATAGCAATTAAATTTATGTTCCACATATAATCCACACATGGAATT TCATTATTTGAAAGTAAACTTTCATTATGGTAATGCGCCGGTGCAGTGACGGGCTAAGAATTTT ATCCTTTTGAACACTTGCATTTTTGTCTTAGACAAACATTTTCTGCTACAATTTATTTAGGTTTTA ATTTGTCTAAACATTTTCAATCAGATTTATGAGAACTTTTTTCAACTTTTTTTTACTTTGCAGACA CGGTCCGCATGTAAGTTTTACAACATTAACTTAGAATGAATTGCTTATAGCTTACACTCAAAATT ACCTAAGTTCCACATGCGATCCACATAATATCCACATGCGATTCACATAAAATCCGCTTGCAAT TTTTTGCTTATTGATTTAAATTTAAGTTCTCTACACTAATTACACTTTTTTTTTACAAATTGACCA AAGTTCCACATACGCTCCACATTTAAAATTTATGAATTTTTTCTTTCTTAACTTTAAAATGATTTA TCTATGTGAAAAAAATATTTTCCACAAAAAGTCGCTGACTTCAACAAGCGATCCACATACGTTC CACATAAAAAAAATCATACCACGCCATAAGATACACTTGCACTTTAATTAAATGCAGTTTCAGG CAAATTTCATTAGCGCTCTATACACCTCACTTGGCGCATACGCAAATGAAATACCCTATATTATA ATACTAAATTTAATATACTCTTTTGATTTCTATTTCTATAAATATTTTTAAATTTAATTAATCTCT TAAGTTTTCAAACCAAAGAGTTACTTTCAGCGAAAAAAAAAAAAAAAAAAAAAAAAAAAAAA

$>$ Cc-omb_v.1-CDS (362bp) in pCR ${ }^{\oplus} \mathrm{II}$

CAGAAGGTCGTCTCATTTCACAAATTAAAATTGACCAACAATATTAGTGATAAACATGG ATTTGTAAGTACTACGATACTCAACTCAATGCATAAATACCAGCCACGGTTCCATTTGGTGCGA GCCAATGATATACTCAAGCTGCCGTATTCAACATTTCGGACGTATGTTTTTAAGGAGACAGAAT TTATTGCCGTTACAGCATATCAAAATGAGAAGATTACTCAATTAAAAATCGATAACAATCCCTTT GCGAAGGGTTTTCGTGATACCGGCGCTGGCAAGCGGGAAAAGAATTGTTACAGGCAAGCGCTC ATGTCGAATCGCGGGTCCGATTCGGACAAACTCAATCCTACACATGT

$>$ Cc-salm-CDS (1463bp) in pCR ${ }^{\oplus} \mathrm{II}$ 
CCTCCGAGACACTGAAGCTGAAGGAGCTAATGAAGAACAAGAAATTGACAGATCCGAAT CAGTGTGTCGTTTGTGATCGTGTGCTGTCGTGTAAGAGTGCTCTGCAGATGCACTACCGAACAC ATACTGGTGAGCGGCCATTCAAGTGTCGCATTTGTGGACGCGCTTTTACCACCAAAGGCAATTT GAAGACCCACATGGCCGTGCACAAGATACGCCCACCCATGCGCAACTTCCACCAATGCCCTGTT TGTCATAAAAAATACTCGAATGCATTGGTACTGCAGCAGCATATACGCTTGCATACCGGCGAAC CGACGGATTTGACGCCCGAACAAATACAGGCGGCCGAAATACGTGATCCACCGCCATCGATGA TGCCGGGTGCCTTCATGAACCCTTTTGCGGCGGCTGCCTTTCATTTCGGTGCTATGCCAGGCGC GGCCATGGGGCATCATCTGGGACCCCACAACGGCACAATGGGCTCAGAGTCATCGCAGGGAGA TATGGATGAAAATATCGATTGCGGTGATGACTACGACGATGACATCTCCTCCGAACACATGTCC AGCGCACACGATCTGGACGTGAGTGATCGGCCGAGATCGAGTGATGATTTCAAAGGTTTGCTCT TCGAACAAAAGTTGCGCATCGATGCCAGTGGCGTTGTGAATACCACACCACGTCCGCACTCCAC CGCTAGCAATGCAAATTCGAATAACTCTGAGCCAAATAGCCCCAACTCCGCGCCACGTCACAGC TCGACACGCGCCAGCTCGCCGGCACGTTCCATGTCTGAGGCATCACAAGGCGCGTTAGACCTTA CACCACGTGCACTGCCAATGCAAGGTAACAAAAGCAGCTCACAATCACCAGCGCCCACAACATC CACAGCACAGGCTGCCGGTCGCAAGACACCTATTTCGCCACCGCGTAATAGTAGCGCCAACAGC AGTGCGGCTAGAAGTCCCCACCCTGTGCCATCACAGAAACCACACCCGCAAATAAGCCCTGCGC TCACTTCACCGCTTGTACCCACCTCAGCGGTCGACTGCTTACCCCCTGTGCTGCACCATCACTTA CAACAGCAGCACCAACAATTGATGCAACAGCAAGCGGTTGCGGCGGCAGCAGCTCATGCTCAA GCACAGGCGCAACATCATCACCAACAATTGCAACAACATGCCGTCGCTATGCATGCCGAGCAAC TGCGGCGTGAGCACCAAATCAAACAGGAGCATGCGGTGCATCAGCATCACTTGCGTCAACAGC AGCATCAACACCAACAACAGCAAGAGCAGCATCCATCGGCGCATTCACCCCATCAGTTAGCGTT GCCTCCACCGCCGCCGCATATCGCTCACCATCTGCAGCAACAACAGCATCAGGCAGCAGCCGCC GCGGCCGCCGCAGCACAACAGCAACAACAACAGCAGTCACCAGGGGCTATGCCACCACAAGG G

$>$ Cc-salm-CDS (1247bp) in pCR ${ }^{\oplus I}$

CCTCCGAGACACTGAAGCTGAAGGAGCTAATGAAGAACAAGAAATTGACAGATCCGAAT CAGTGTGTCGTTTGTGATCGTGTGCTGTCGTGTAAGAGTGCTCTGCAGATGCACTACCGAACAC ATACTGGTGAGCGGCCATTCAAGTGTCGCATTTGTGGACGCGCTTTTACCACCAAAGGCAATTT GAAGACCCACATGGCCGTGCACAAGATACGCCCACCCATGCGCAACTTCCACCAATGCCCTGTT TGTCATAAAAAATACTCGAATGCATTGGTACTGCAGCAGCATATACGCTTGCATACCGGCGAAC CGACGGATTTGACGCCCGAACAAATACAGGCGGCCGAAATACGTGATCCACCGCCATCGATGA TGCCGGGTGCCTTCATGAACCCTTTTGCGGCGGCTGCCTTTCATTTCGGTGCTATGCCAGGCGC GGCCATGGGGCATCATCTGGGACCCCACAACGGCACAATGGGCTCAGAGTCATCGCAGGGAGA TATGGATGAAAATATCGATTGCGGTGATGACTACGACGATGACATCTCCTCCGAACACATGTCC 
AGCGCACACGATCTGGACGTGAGTGATCGGCCGAGATCGAGTGATGATTTCAAAGGTTTGCTCT TCGAACAAAAGTTGCGCATCGATGCCAGTGGCGTTGTGAATACCACACCACGTCCGCACTCCAC CGCTAGCAATGCAAATTCGAATAACTCTGAGCCAAATAGCCCCAACTCCGCGCCACGTCACAGC TCGACACGCGCCAGCTCGCCGGCACGTTCCATGTCTGAGGCATCACAAGGCGCGTTAGACCTTA CACCACGTGCACTGCCAATGCAAGGTAACAAAAGCAGCTCACAATCACCAGCGCCCACAACATC CACAGCACAGGCTGCCGGTCGCAAGACACCTATTTCGCCACCGCGTAATAGTAGCGCCAACAGC AGTGCGGCTAGAAGTCCCCACCCTGTGCCATCACAGAAACCACACCCGCAAATAAGCCCTGCGC TCACTTCACCGCTTGTACCCACCTCAGCGGTCGACTGCTTACCCCCTGTGCTGCACCATCACTTA CAACAGCAACACCAACAACAGCAAGAGCAGCATCCATCGGCGCATTCACCCCATCAGTTAGCGT TGCCTCCACCGCCGCCGCATATCGCTCACCATCTGCAGCAACAACAGCATCAGGCAGCAGCCGC CGCGGCCGCCGCAGCACAACAGCAACAACAACAGCAGTCACCAGGGGCTATGCCACCACAAGG G

$>$ Cc-wg-5'-UTR (934bp) in pCR ${ }^{\otimes} I$

TTTGGCGATAGCATTGAATCGGGTAACAGTATTGTTCGTACTGATAAATAATTTGGCATT CATCTTGAAATTTTTAAAAGAAATTTAATGCGCGTTTTAAGCCAATCATAAATTAGTTTACTAAT CTAAAATAGCAGTAATTGTTATTTATATTATGTACACGTATACGTACATACATATATATATGATG TACGAATCAATGCTCGTCACATACGTTGAATGATCTAATTATATTTTATACGCAATCAGATCACG TGTGAAAATTGTAAAATTTTGTGAGTGTGAATATTATCAAAATTTAAATCTACATACTACCTACT ATGGATTGGCACGGAACAAGTGTTGTGTAATTAGCATTACTCAAAAAAGTAATTGTAAATAGTG GAAGATTGTAAATAGTATACAAATAAAAGCGTCTCATATCAATGGTGTGAATGTTTGGCTAACG AAGCTAGCTAAACTAGAAACAACTTTCATTTTAACTAAAACACTAAGATACAAACATAATTGGA ACAATGGATTTATCAACAATGCTCTACGTATATTTACTAGTAATGTGTACGTACGCAACAACGG AGAGTAAACCGAAACCTGGACGTGGAAGAGGATCCATGTGGTGGGGAATAGCTAAAGTGGGGG AACCAAACAATATATCACCACTTAGTTCTGGTATTATGTACATGGATCCAGCTATACATTCCACA CTACGAAGAAAACAACGACGTCTAGTTCGTGACAACCCTGGCGTTTTAAGTGCTCTTGTGAAAG GAGCGAATCTTGCCATAAGTGAATGCCAGCATCAGTTCCGAAATCGACGCTGGAATTGTTCAAC TAGGAACTTTTTACGTGGGAAAAATTTATTTGGAAAAATTGTCGATCGAGGTTGTCGTGAGACT GGCTTTATTTATGCCATCACCAGTGCCGCTGTCACACA

$>$ Cc-wg-3'-UTR (601bp) in pCR ${ }^{\circledR} I$

TGCAGCCAGCCCGAATGCTTTTAGTGCTGATCGCATGTTAAACGATCACACTCCTGATC ATATATTAAAAACTTATTCAAGCAAACCTCCATCGAATACCATAAATAGCTCAAATAGTTTGCCC CGAAGTTTGGTCAGAAACCGTGTTCGTCAGAGGAAAAAGAACAACAGATACAATTTTCAATTGA AACCACAGAATCCCGAACACAAAGCACCTGGTCCTAAAGACATTGTTTACCTAGAACAATCACC AAGTTTTTGCGAGAAAAATCTTCGCTTAGGTATTCAAGGAACTCATGGACGTCAATGCAATGAT 
ACATCGATCGGAGTTGATGGTTGTGATTTGATGTGCTGTGGTCGTGGCTATCGTACACAAGTCA TTGATGTCATCGAACGCTGTGCATGCACATTTCACTGGTGTTGTGAAGTCAAGTGTAAAACATG CAGAATGAAGAAGACAATACATACATGTTTATAAGTAATGACGTGAACTTTTCAAATCACCGCC CGATACCCATTCAAAGCTGCCTATGCCTGAATATTTTCTAAAGCAAAATTCAAACGTTTCTAAAA AAAAAAAAAAAAAAAAAAAAAAAAAAAA

$>$ Md-ap-CDS (1310bp) in pCR ${ }^{\oplus} \mathrm{II}$

GTCCAACACCACCTGTAGCACATCAAGGAAATGCTGCACATTGCGGCAGTGCTGCAGGT GCAAATAACAGTAGTAATAACAACAACAATAATAATAACAACAACAATAACAACAACAACAGT AGTAGTAATAGTCATAGCAATCAATTCATATTCCGAACATCAGCCTGCTCAACATGTCCCGATA TCTGTGAACACAGTACAAAGCCGTTCAGTGCAGCGGCGGCCGCCTATTCAGTTGCATTTAGAAG CTATGAAACAGCCGAACAGGCCACCTTCGATGACAGCTCCATCAAATACGCCATCAGTCGAGAC CAAACGGAGTGTCCGGACGATCTCAACGATGAGACCAATTCGGGTATATCCTTCAAAACGGAAC CCTATGGACCACCCAGTAGTCCCGAGTCGGCGACTGAGACAAAACCAAATTGCAGTCCACCCTG TGCCGGGTGTGGGCGTTTGATACAGGATCGTTTCTACCTCTCCGCTGTGGACAAACGGTGGCAT GCCAGTTGCCTGCAATGCTATGCATGTCGACAACCCCTCGACCGGGAATCTTCGTGTTATTCGC GCGACGGTAATATTTACTGCAAAAGCGATTATTATAGTGTTTTTGGTACACGACGCTGTTCCCG CTGTCTGGCATCCATTGGAGCCTCCGAGCTGGTAATGCGAGCGCGAAATTTAGTATTTCATGTG ACCTGTTTTTGTTGTGTGGTCTGCCAGAGTCCCCTGACAAAAGGTGATCAGTATGGGATAATAG ATGCTCTCATCTATTGTAGAACCCACTACACCATGGCCCGCGAAGGTGACACCGCCTCAACCAG CCTCGGCCCAGCAGTGGTGCCCGGTGTGGGCGGTGCCAGCAGCTATCCGTACACAAGTCAATTT GGTTCGCCACCGAATGATTCGTCGAGTCCCCACTCGGATCCGACACGCATTGTTCCTAGTAGTA TATTTGCCTCCGCCTCTCACGTCATGACCGGTCTTCCACAGCCGCCCCGGCAAAAGGGCCGGCC CCGCAAACGTAAGCCCAAAGATATTGAAGCCCTCACTGCCAACATAGGTATGCAATATTTAAAC ACTGAGTATTTGGACTTTGGACGCGGTTCGCACATGAGTGCCTCGTCGCGTACAAAACGGATGC GAACGTCCTTCAAACACCATCAGCTGAGGACCATGAAATCGTACTTTGCCATAAATCACAATCC CGATGCCAAGGATCTGAAGCAGCTGTCGCAGAAGACAGGTTTACCAAAGAGAGTATTACAGGT GTGGTTCCAAAATGCCCGTGCCAAATGGCGTCGCATG

$>$ Md-dpp-CDS (1133bp) in pCR ${ }^{\oplus} I$

GGTCCACGCACCTATAGCAATAACGAGGTGATCGGTGACAAACTGAAACCGGATCCCTC AACTCTAGTCGAAATTGAAAATAGTTTATTGTCGCTATTCAATATGAAGCGGCCGCCGAAAGTC GATCGTTCCAAAATCGTCATACCGGAGGCCATGAAACAGCTGTATGCCCAGATCATGGGCCATG AATTGAATTCTGTAAATATACCAAAACCCGGTTTGCTGACCAAGTCGGCGAATACAGTGCGAAG TTTTACGCATCAAGATAGTAAAATCGACGATCGGTTTCCTCACCATCATCGGTTTCGTTTACATT TCGACGTGCGCAGCATACCCGCCGAGGAGAAACTGAAGGCTGCCGAACTGCAGCTGACACGTG 
AGGCCATCAGTCATGCCACCCTCAATCCCCGCCTGGCCAATCGCACCCGCTACCAAGTGCTGGT CTATGACATAACACAGGTGGGTGTGCGCGGCAAGCGGGAGCCCAGCTATCTGCTGTTGGACAA CAAGACCATACGGCTGAATAGCACAGAGACGGTGAGTTTGGATGTCCAACCTGCTGTGGATCG GTGGCTGGCTTCACCGAAGAAGAATTTCGGTCTATTGGTGGAGGTGCGGACGTCACGCTCCCTG AAACCGGCACCGCATCATCATGTACGCTTGCGCCGCAGTGCGGACGAGGAACATGACGCGTGG CAGCGCAAACAGCCCCTGCTTTTTACATACACCGACGATGGACGGCACAAATCGCGTTCCATAC GCGATGTCAGCAATCGTTCGAAGCGAGCTGGCCACAATCGTCGTTCGCATCGGCGAAAGAATA ACGAGGAGATCTGTCGACGCCATTCGCTCTATGTGGACTTTACGGATGTCGGGTGGAGTGATTG GATTGTGGCGCCGCCGGGCTATGATGCCTTCTATTGTCACGGCAAATGTCCATTTCCGCTAGCG GAACATTTAAATTCGACAAATCATGCAGTGGTCCAGAATATGGTGAACAGCATTAATCCGGGGA AGGTGCCAAAGGCATGCTGTGTGCCAACACAGTTGGAGGGCATATCGATGCTCTATTTGAATGA TCAGCGTACGGTTGTGCTCAAGAATTACCAGGATATGACAGTGGTGGGTTGTGG

$>$ Md-en-3'-UTR (666bp) in PCR'II

CAAAGTCCCCAGCCCATCCCGCCACCGTCGGCAATTAGCCGTGACTCGGGCATGGAAAG TTCGGATGATACAAAATCAGAAACGGGCTCCACAACAAATTCGGAAACTGGTAAAAATGAAAT GTGGCCAGCCTGGGTATTTTGTACACGTTACAGTGATCGTCCCAGTTCTGGTCCCCGTTATCGTC GCCCCAAACAACCCAAAGATCCCAAGGCGACCGATGAAAAACGACCAAGGACTGCATTTTCCG GCGAACAATTGGTGCGATTAAAACGTGAATTCAATGAAAATCGCTATCTAACCGAAAGGAGAC GCCAACAATTAAGTGCTGAATTGGGTTTAAATGAGGCCCAAATTAAAATCTGGTTCCAAAATAA ACGCGCCAAAATCAAGAAGTCCACCGGCACCAAAAATCCCCTGGCCCTGCAACTGATGGCCCA GGGTTTGTATAATCACACCACAGTGTCGCTGACCAAAGAGGAGGAAGAACTGGAAATGAGAAT GAATGGACAGATTCCATAGATTTCCATAACACCCAAAAAAAAAAAAAAAAAAAAAAAATAAAA CCAGATTAGTGCAATACCCTGAAAGTGTAAATAGTGGTTGAGAAAAGAGAATCCAAGGAGCCC AAGAACAAATTACAAAAAAAAAAAAAAAAAAAAAAAA

$>$ Md-hh-5'-UTR (749bp) in PCR ${ }^{\oplus} I$

CGCGGGCGTCGCCGTCGTTGTTCTGCGAGTGTTAGTGGCATAGAATAGAACTAAGAAGA CATGTTAGATTAACGAAAATTAAAATTTGCAAAAAATAAAAATTGAAATTTTATCAAAAGAAAT ATTGCGATTAAATGAAAAAATACTTGAAATGTATGGTCATTGAAAAATTGGAAAATAAATAATG GATTTGAAAGTTAATTAAATTTATAATCGATAAGAAAAAAAATCCATTACAAAAACCCGATTGT GAAAAGTGAGTGATTTTGTCCAAAAAAAAAAAATTGTTTAAAAAAATAAAAACAAAAATATCCT CAAAAATGGAATTTGAACAGCGTGTACCCTGCATTGAAGGTACCACCGACATGTCCTGTGATAA ATGTCACATTTGTGCCAATAACAACAAGAAATACCACCTGTCCTCGTGTGCAACGCCCAGTTAC ACCTCAAGCCAGACCAGCGAGAGCATCAGCATGAGCGATATGGAATTCAATAAAACCTTTCATT GGTTGCCAAACGCTAATTGTGGATTAAGTTCAGCATCTTCAACACCAGCATCCTCATCATCGTA 
TTTTACCTTGCGTCGCATTTGCATGCTATTGCTTGTGGCCAGCCTCTTCTCGTGTGCCGCCGGTT GTGGCCCCGGTCGGGGTATTGGTGGTCCACGTCGTCGAAGAAAACTAACACCTTTGGTGTTCAA ACAGCATGTGCCAAACATGTCGGAACAGACCCTGGGAGCATCGGGCGTC

$>$ Md-hh-3'-UTR (408bp) in PCR ${ }^{\circ}$ II

TGTGGTCAACTCTGTGGCTGCCTCCTGTTATGCTGTGGTAAATAGCCAATCATTGGCCCA TTGGACTTATGCTCCCATGCGTTTGTGGGCAATGCTAAAATCTTATATACCCTCAACGAGTACTG CTCAAATGCAAAATGGCGTCCATTGGTATGGCAGGACCTTGTATGGCATTAAAAATTATTTGGT ACCCAAGTCATGGAGGCATTGATAGGAGCCAAATGAAATTTTATCAACGAAAAAATCGCCTTTC TAGTTAGCTGTCCAATTGTTGGTGGTTGCCTTCTATTAAAAAAAACGAAAAACAAAAAACCTAG GCTATAAGTAGGAAAAACAATTAAATGGAATTTTTACTACCTGATTAATACAGAAACAAATCAA AATGCGCATGCGCTTAGTATTCTTTTG

$>$ Md-kni-3'-UTR (155bp) in pCR ${ }^{\oplus} I$

CCACACCGGAACCCGCCACCTTTGCTGCCAAAATGGAATCCCTCTCTCCCGTCTCCGTGT GCTCCATTGGCCATGAAACCACCAAATCATCGCATTCCTATGAGGAACCCATGAACCAAGATGG TCCCATGGATTTGAGCATGAAAACCTCCCGC

$>$ Md-kni-3'-CDS (1603bp) in pCRII

GAAATGATGAACCAAACGTGCAAAGTATGTGGCGAACCTGCTGCCGGCTTCCATTTTGG AGCCTTCACATGTGAGGGATGCAAGTCCTTCTTTGGCCGCTCGTACAACAACCTCTCCTCCATCT CAGAGTGCAAAAACAACGGCAAATGCGTAATTGACAAGAAAAACCGCACCACCTGCAAGGCCT GCCGTCTACGCAAATGCTACACCGTTGGCATGTCCAAGGGTGGCTCACGCTATGGCCGTCGCTC CAACTGGTTCAAAATCCATTGTCTGCTGCAAGAACAACAACAACAGGCTGTGGAGGCTGCCACT AAGGCGGCTGCTGCCAATGGATCTGTGGTGCCCCCAAATCCCGCCGCTGCTGGACCCTCGCCTT TCGGTGATATGGCTGCTGCTGCTGCCGCCGCTGCTGCCCAACAACAGTTCAATGCCCAGCGTTC CTCACATTTGGCTGCCGCCGCTGCTGCCGGAGCCGGCCATTTGGGCTATCCTTCATATCTACCC GAAATGTCTGCTGCCCCCTTTATGTCGGCTGCCGCTTTGCCCTTCTTCAGCATGATGAACGGTAT GCCACCCATGGGTGCTGCTGCCTCACCTGCCTCAGCCTTCCAAATGCCACCACATCTACTGTTC CCCGGCTATCATCCAGCTGCTGCTGCTGCCGCCGCCGATGCTGCCTATCGCTCGGAAATGTACA AACATCGTCAATCCGTGGATTCGGCCGGCTCAGCGGAATCTCTGTCACGTTTCTCACCCACAAA TCACTCAGTACACTCCCTGACCCATGAGGAACCCCAACAGCAAGCCACCCACTCTGGACGGCAA TCACCTGAACTGTGTGTGTCCGGAGATGATGATGTCATGCATCACTCACACTCCGTCTCACCTG TGTCCACACACTCCATGACACATCAAATTCACAGTCCCATTGCTCTACACCCCTCACCACTGCCA CAGGTTGCCTCACCCCACATGGCAGCCATGAGCAACAATGCCACACCGGAACCCGCCACCTTTG CTGCCAAAATGGAATCCCTCTCTCCCGTCTCCGTGTGCTCCATTGGCCATGAAACCACCAAATC ATCGCATTCCTATGAGGAACCCATGAACCAAGATGGTCCCATGGATTTGAGCATGAAAACCTCC 
CGCAGCTCGGTGAACAGCGACAGTGATATGCACAGTGAGGCCAGCGAAGAGGAACGCCAACAG AACATGCGTCGCAAATTCTATCAGCTGAATGAGACCGAGAACTCGGAAAGCTCCTCGGATTCAG AGGCCTCCAAGAGGCAAAAACTGGCCGAGAACAACTATCATTTTGGCAATCACAGCCACAGTCA AAGAGGTATTGTATGTGTTTAAGGAGAGCAAAGCACAAAAAAAATAATTTTACAAAAACAACAA CAATCATACCAAAATCACCTCTCACCAGAAAAAAACACACAACCAATCAACCAACCATTTATAG TCAATGCAAAAAAACATTAATTTTTTTAGTTAATAATTTAATATTTTTACCCCACCTTTTTTTCTT TAAACATAAATTGTAAATATTTGCAGGGAGCCCACTGTCCCACTTGGCAGTCTGCCCCTGCACC AACAA

$>$ Md-mad-3'-UTR (444bp) in pCR ${ }^{\oplus} I$

TGGACCGCTGCAGTGGCTGGATAAGGTCCTAACACAAATGGGTTCACCTCACAATGCTA TAAGTTCGGTGTCATAAAATGCCGTTAGCTACAGATTTCGTTTCACTAAATATAAGTTAATAGA GTTAGAGTAAGTTTTAAGAAAAAACAAACGAATGAAGAAATAAGTTCAAATTGAGACACACCA ACCACTTACAGTTAAGAAAAGTAACAGAAAAGACCATCAACCTTACTAATTTGAGAAATTCTAC TTTCCAATTGTATCATCTGTAGACAGACGGACGGCCGATTGTAAATTTTGAACAGAGTTGTGAG ACCAGCCTTATTTGTGATTTTCTAAACACTGTTTACACGGATTTATCTCCGACGTAGGATACGTG TCAAAAGTACTGCTAGTATTTCACACTCAGTAGTATATGCTTTTAATCTGGAAAATATCCCGCGT $>$ Md-mad-3like-5'-UTR (784bp) in pCR II AGTGTTGTATTTATGTTCAGAAGCGACAAAATTATTTTTATTTTTCATTTCTGTTCGGTGA TAAATTCATTTAATTATTAGAAATTTTTGCTGGCAAAAATATGTTAATTGAACCAGAATTGTAAA ACAAGTGCACGAGAATTGAAATTTGCTAAAATTGCGGTGAACTAGAAGTGATTAGATAGCAAAA TATCGTCTGTGAAATATTCTGTGTGTGGTTAGTGAACGTCTCGTTGTCGTTGTCTTCTTGGTCGG TCCAGACAGGCAGACAAGGTAGACAGAATTAAGAGGGGGTGCTGACAGACAAAGGAGGGAGA AAAGAGGGAGAGCGAGATAAAAGAAAAATACGAAAAATTAGAATAACAAGCAAAACATACCTA ATAAGAGACAGATACAAGCAAAAACTACGCGGATGCTATAATCTGTAACATTTGGAATTTTTTC ACTCAGGGGGTATCAATACAAACTTAAACGTGTAATAACGTCCAAGGACGATAATCCAGCTGCC AACTGCTGTGCTAACAACCAAAACAAAACCAAGCAACAAATTATCCTCACCGCTGTTAACGTGA AATGTTGCCATTTACACCACAAGTCGTTAAACGTTTATTGGCATTAAAAAAAGTGTAACGAAGA TAGCGTTGAAGGCAAGTGGTCCGAAAAGGCTGTTAAAAATCTAGTTAAAAAGATCAAAAGAATT CCTCGCTAGAAGAGTTAGAACGTGCCATATCAACACAAAACCCCAATACAAGGTGTGTTACCGT ACCGCGCAGCAAACCCACTG

$>$ Md-mad-3like-3'-UTR (288bp) in pCR ${ }^{\oplus} \mathrm{II}$

TCGCAGTCCGTGTCGCAGGGTTTCGAGGCAGTGTACCAGTTGACACGCATGTGCACCAT ACGTATGTCGTTTGTCAAGGGTTGGGGTGCCGAGTATCGACGTCAAACGGTCACATCCACACCC TGCTGGATTGAATTGCATTTGAACGGTCCGCTGCAGTGGTTGGATCGAGTTTTGACACAAATGG 
GTTCGCCACGCATGCCATGTAGTTCCATGTCTTAAAAAGTAAGAGGTTTTTAAGGCATATCTTTT AAAAAAAACCGAAAAAAAAAAAAAAAAAAAAAAAAA

$>$ Md-mad-4like-CDS (1536bp) in pCR II

CATTTGCTGCGCCTCTGAACTCTGTGAGAATTTGGAAAAATTTCTTTTGATTAGAAGGAA AGAAAAAAACACGGAACCATTTTCTTCTATTGTAATTGTGGGTCTTCTCGACCCTCTGCGGCTAT TGGAATCGGTTTCGTTATTTTCATTTGCTATTTACGCGAAAAAAATGTCAAAAACTGCCTCAAGC TGTGTTATTTGTGGCATAAAAATAGAATTTCGCGAGCGATTGTGTAAAACAAAGAAATCTATCA AAGTATAAGAGTAAAAGGTGGGAGTTGTATATCTTGTGTGTATGTTTTGGTCACCTTCGCCTTC GTGCGGCGCGGCGCTTTCAACAAACAACAAAGGAAAAAAAACGAAAAGGAAAAGCAATTGTGT TTTGCTATAAGAAAATCCAAAAGGATATTAAAGTGAAGTGAAAACAATACATAGTTGTGCTGTT AAATAATTATTGACATCGATTGCTTGGTGTTGCACTCTCTGTTTTGCTCGGCTCCAAACACTCTC TCTCCCTCCCTCTGTTCGAGTTGAGCTAACCGAACAGACAATTATTTTGTAAATTTGTGGCCTTT CCTGTATCAATTTGTGGCCATCGTCGAATTTCGGCTATTGTGACGGCGATTTTACAAACGCCGC CGGCAAAGTGGATCCCCGAGGTTTAGGACCCCAGGACTATGCCGGCATGGTTGGTTTGCCGGG CAGTAGTGCTGGGGTTGTACAAAATGGTGGACCGGGCATGGGTGTGCCACCCGTTGGTCCGGG TGGTCATATGTATCCGGGCGTTGGACCACAAGATATTCGCGATATTGTCCAAATGCCTCCACCA CCACCCTCGAATGCACCCACATCGGCCGATGCTTGCCTGAGCATTGTCCATTCGCTGATGTGCC ATCGTCAGGGCGGTGAAAGTGAAGGATTTGCCAAACGTGCCATTGAATCGCTGGTCAAAAAACT CAAAGAGAAACGCGACGAATTGGATTCATTGATAACCGCCATTACAACCAACGGTGCACATCCC AGCAAATGTGTTACAATCCAGCGTACTTTGGATGGACGCTTACAGGTTGCCGGACGCAAAGGCT TCCCACATGTCATCTATGCCCGGATTTGGCGCTGGCCCGATTTGCACAAGAACGAATTGAAACA TGTTAAATATTGTGCATTTGCTTTTGATCTCAAATGTGATTCGGTTTGTGTGAATCCGTATCATT ATGAACGTGTCGTTTCGCCGGGCATTGATTTGTCCGGTCTCAGTTTGCAATCGGGGCCAAGTCG CCATGTTAAAGATGAATACTCAGCTGGGCCGCTGGTGGGTGGTATGGACATTGATGGCAATGAT ATTGGGACAATACAGCATCATCCATCACAAATGGTGGGGCCACCAGGCGGAGGTTATGGCTATC CCCAGGGACCGGTTAATGACCCGAATCACATGAATGCCGCCGCAATGTATGCCGCTGCAGCAG CAGCTGGTCGTACGATACCAAAAATTGAACCAAATGAGGCAGGTCCACGTAGTTCGTGGATGG TGCC

$>$ Md-mad-6like-CDS (2093bp) in $\mathrm{pCR}^{\oplus} \mathrm{II}$

GGCTACATACGACTCCAGACACCCGCCACCACCACCACCAACACCGTCGCCCGTATCGA AATACAAAATCAATCCAATTAGTTAAGGCGAATAATGCAAATAGGATAAGATCGATGTTACCGC TACATTTAATGTCTCTCCATGAAATTTCAAAGCGAGAAGAAAGAACTATGGCGTTATGCGTCAA AAAACCTAACAACACCTGTACGTGGGACGACCAGACAGCCACCACCGCCGCATCGCCTTCCACC TCAACACCACCCTCACGAACCGGAGCGGTCACTGTTGAGGCAGCAGACCTTCTATTGCGACGTC 
TCTGGCGAGAGCTCCAGCACCATGTCGCTGCGGCCACAACTGAGCACACCTCCGCCGCCCTACA GTGCCACGGCCGTGGACATGGACTGTGTGAATCCTCGAGACTATTGTCAAACGTTTCGCCAGCC GCAGGCCTATGTAACGGACGGAGAAACGGAAACATCGCTGTCGGCGACCGAGTCCGAAACCGG TTACGGCCAGAGGCCAGGTGGCTACAGCTACGTAAATACTGGCGACATAATGCACGTCGACGA GTACGAAAAACTGAACGAGATCAGTTCAGGCAGCAGCAGCAGCAACAACACCACCACCTCCTC CACCAACAGCACCAGTACCTTTCATCTGACGAAGGATCTACCCAGGAGGTTGGAGCGAGCAGT GACCACACCAAGGCGTACGCAATCGAAAACGAACATTTCCCTCAATCCCGTTGCCACGACTCTT GTAAATTCGGCAACAACCCTGACCTCGGCAACGACTTCGTTTGCTGCCACAGCAGCAAAAATAC TCAGGAATTGTTGTGGCGGAGGTTTTGGTTCCTCCAATTCCATTGTGACCGCAACAAGCAGTAC CAACCACGATCCCTTCAGCCTGAACCCCACCACAACGATGACTACGACGACGGTGGCCAACAAC AACAACAATTCCACCATGCCCTGCAACAAGGAAAGCTACGAAAACTCGAAAAACATGGCCACG TCGGCGAAAACGTATTTCGATGCTCTAATGAAAGAACTCAAGGCCCGTCAAATAACCAGCTTGT TAAAGGCTGTGAAATCGCGTCACGAACCCACAACAAAGTCGTCGACGGCGGCAGCGTGCTCGT CCAACAGCGTGACAACATATCAAACCAATTGCATCTTGATGCGTCGTGCCGAAATACTCGGCGA AGAACCGTATGTGATAGTGTGTCGCCTGTTTTTGTGGCGAGATTTGAACAATTCCTCACAGCTG AAACGCTTACCCGTATGTCCAAACGAACGCGACCCAGTGTATGTGTGTTGCAATCCCTTGCACT GGAGTCGCATTCTCGAAACAGAAAGTGCCCCGCCTCCATATCGCAGCCAAAGGATGGACAGAA ACGAAGGACTACCTTTTCAAAACTACGGCAACTCCACATCTTTCGGAAGTTCAGATGCCGACGG CGTTTCCAGTATACAAAAACCCAAAACCGATTTTTATGTTCGAATGACAGAATCGGTTACCACT GACGGTGAAGCCAGTCGCTGTTCACCGAACTGGTGCGAGGTGGCCTACTGGGAATTGGGTCAA CGGGTCGGTGAACGTTATCCCGCCGAAACACCAACCATAAATATCTATTCCGAAAAACCCTACA GCAGTACTAATGGTGATATAGAAGGTATGTGTCTGAAGGATTTAATTGAGAAGCGTACGACACC ATCGCCAAGTGATGTACAAAATACGAGACAAAAAATAGGTTTAGGTGTTACTCTAAGTCAGGAG ACCGATGGCGTTTGGCTATATAACCGTGCAACTGTGCCAATATTTGTATACTCACCCACCTTGAC GGATAGTTTGTCTCGTGTTTATCGCGTGGAACCCGGAGACTGTCTTAGAGCCTTCGATATATAC AAGACCCACACTCTCGTCTATTCCGATCAATTTCCAGGCGTTCAAACCGGACCCATTGATAAAT TTAGCATGCGCATTAGTTTCGGCAAAGGTTGGGGTTTTTCCTATAAACGGCCGGATATAACGCG ATGTCCATGCTGGTTGGAGGTACTCTTTCGGCCACAACGGTGACAACAATTCGTCGC

>Md-omb_v.1-5'-UTR (183bp) in pCR ${ }^{\circledR} I$

ACGCGGGAACATTTGTCTTCCACTCTTCGTTATGACCATTTCCGTGCCAAGTTTGTGAAAT TTCTCCCACAGCTCCTTACCCTCCAGCGTCACCTTGGGATCATCGACCACACCATCCTCCTCCGG CTGCAGAGCACGCAGCGGACGCATGGCCGGATGCAGTTGATGGGCCACCGCTGAGGC

>Md-omb_v.2-3'-UTR (699bp) in pCR ${ }^{\oplus} I$ 
ACCCACCACGCCGCCCTCCCTTATGAAACGTACCAACTCACCGTCAGAGCTCAAATCGA TTGAAAACATGGTCAACGGGCTGGAGGTGCAGCACAATGGCAGCGGCTGCAGCACCAGCGGGT CCATGGATGATTTGCACCGCAAATCGCCGGGCCTGCATGGGGACCAGTGACAATTGTATGACCG GGACGACAACGATCGCTCATCTGTCATAGATCTGGATGATGTGTGACAAGTTAGCTATTCGTTG CAACTGCAGCCCGTACTAAATGCCTGGCGTCAGATCAATGGAATTGATGGCATCACAAACAACA GCAACAACAGCAGCAGCCATAATCATCAGCAGCCGCAGCAACAACAAAATCATCATTCGAATC GTTATTCCATTGATTAGCCGCATAACTAAACCAGACCACAACAACATCGCCGAAAAAGGTAAGG AAACAGCTTTCCAGGATCAAAAAAATCATTAAATGCTGTTTCCCCCCACAATTTACTTTAACCTT CAAAAGTTCTGACTGTCAGACCCTAAGTTTAATTTTTATTTTTTTTTTAATTTTTTTTTATTTTAA TTTTGTTTTCAATTAATTCAAACCAAAGAACGTATCTTACTTGTAATCTTCTTTAGCTGCTACTTT TTATCTCTTATTCTGTTTTCTTACTCAACACAAAAGAAAAAAAAAAAAAAAAAAAAAAAAA

$>$ Md-omb_v.1-CDS (680bp) in pCR ${ }^{\circledast} I$

GTCGGCTTTACTGAGTGCCTCACATTCGGCTGCTGCTGTGGCAGCTGCTGCTGCGGCCG CCGCCGCTTCAACGGGACACGCCATACCACCGCATTCATCGCCCCAAGCTCAAGCGCCCCCACC CACACATCCTCATCACTCACCAGTACCTGGTCACCTAGGAGCCCATTTGCCTCAACAGCCACCG TATTTCCCAGCAGCTGCCCTGGCCGCCCTAGCCGGTAGTCCTGCGGGTCCGCATCCGGGTTTGT ATCCAGGCCCGTTACGTTTTCCGCCCCACCATCCGCATCATCACCACCATCCGCTGGGCACGGC CTATACGACCGCCGAAGACGTGGTATTAGCCTCAGCGGTGGCCCATCAACTGCATCCGGCCATG CGTCCGCTGCGTGCCCTGCAGCCGGAGGAGGATGGGGTGGTCGATGATCCCAAGGTGACGCTG GAGGGCAAGGAGCTGTGGGAGAAATTTCACAAACTTGGCACGGAGATGGTCATAACGAAGAGT GGAAGACAAATGTTCCCGCAAATGAAATTTCGTGTATCAGGCTTGGATGCCAAGGCGAAATATA TTCTACTACTGGATATTGTGGCTGCCGATGATTATCGTTATAAATTTCACAATAGCCGCTGGATG ATTGCCGGCAAAGCCGATCCGGAAATGCCCAAACGCATGTACATCC

$>$ Md-salm-CDS (1436bp) in PCR $^{\circ} \mathrm{II}$

TCAGCCACCAGCTGTTATGTATCCCCAACCCTCGCCTGGATCTCAACACAGCCTTGACCA TTTGCCCACCCCCGGGCAGTTGCCACCTTCGATGGTGCATCATCGTGAGGATTTCTTTGCCGAC CGCTTCCCCATCAATTTTACCAAGACTGATGACAGACACTCGCCGATACGCTCGCCAGCCCATT TGCCGCGTCCACCTTTCTGCAATCCCATGAAACCCTATGACATGGCCTTGTTGCCACGGCCCCA CAGCAATGACAACTCGTGGGAGAATTTCATTGAAATCTCAAACTCCTCGGAAACCATGAAACTA AAGGAGCTGATGAAGAACAAAAAGATTACCGATCCCAATCAGTGTGTTGTCTGCGATCGGGTGC TGTCGTGTAAGAGTGCTCTGCAAATGCATTATCGCACCCACACTGGGGAGAGGCCTTTCAAGTG TCGCATTTGCGGTAGGGCCTTCACCACAAAGGGTAATCTGAAGACCCACATGGCTGTGCATAAA ATTCGGCCACCAATGCGCAATTACCACCAGTGTCCGGTGTGTCACAAGAAATACTCCAATGCCT TGGTGCTGCAGCAGCATATACGTCTGCACACCGGAGAACCCACCGATTTGACACCTGAGCAAAT 
CCAAGCTGCTGAGATACGTGATCCACCACCCACCATGATGCCGGGAGCTTTTATGAATCCTTTT GCCGCAGCAGCATTCCACTTTGGTATGCCCGGTGGTCCTGGTATGCCACCAATGGGTGGTCCCC ATAATGGCACCATGGGCTCGGAATCGTCTCAGGGTGATATGGAAGATCAAATGGATTGTGGTGA GGATTATGATGATGATATGTCCTCGGAACACATGTCCAATTCGAATGACATGGAATCAGTGGAT AGGCCAAAGTCCACGGATGATTTCAAGGCCTTGCTGTTCGAACAAAAGCTAAGGATTGATCCCA CTGGCATCGTAAATGCCGGTTCCCGGCCCCAATCGGCCACCTCCAATGCAAATTCTGTGAACTC GGCACCCAGCAGTCCGGTGGCTTTGAGTAAACCCTTAAATGTTACCCGTTCCAGTTCACCTGCC CGATCGGCATCGGAGACGTCGCATGGGGCTTTGGATCTAACACCAAGGGCGGCTCCCTCAACG GCACGTTCACCACAACCCGTGACATCGGCGAAACGACGATCCCCCTCGCCTTTGAGCAAATCAC CAAAAATGCCACAAATTAGCCCAACCACCAGCCTACCACCCACTGGTGCCAGTCCGGTTGACTG TTTGCCACCAAGTTTACATCGTCACTTGCAACAGCAACATCAACATTTAATGCAATAACAAGCT GCCTTGGCTGCAGCCCAACATCATCAGCAAATGCAGCACAATGCCGTCGTGGCCATGCATCAGG AACAGTTGCGTCGTGAGGTAGCCCAGGCTCAGG

$>$ Md- salm-CDS (787bp) in pCR $\mathrm{II}^{\circ}$

TCAGCCACCAGCTGTTATGTATCCCCAACCTTCTCCTGGATCTCAACACAGCCTTGACCA TTTGCCCACCCCCGGACAGTTGCCACCTTCGATGATGCATCATCGTGAGGATTTCTTTGCCGAA CGCTTCCCCATCAATTTTACCAAGACTGATGACAGGCACTCGCCGATACGCTCGCCAGCGCATT TGCCGCGTCCACCTTTCTGCAATCCCATGAAACCCTATGACATGGCCTTGTTGCCACGGCCCCA CAGCAATGATAATTCCTGGGAGAATTTCATTGAAATCTCAAACTCCTCGGAAACTATGAAACTC AAGGAGTTGATGAAGAACAAAAAGATTACGGATCCCAATCAGTGTGTCGATTGCGATCGGGTG CTGTCGTGTAAGAGTGCTCTGCAAATGCATTATCGCACCCACACTGGGGAGAGGCCTTTCAAGT GTCGCATTTGCGGTAGGGCCTTCACCACAAAGGGTAATCTGAAGACCCACATGGCTGTGCATAA AATTCGGCCACCAATGCGCAATTACCACCAGTGTCCGGTGTGTCACAAGAAATACTCCAATGCC TTGGTGCTGCAGCAGCATATACGTCTGCACACCGGAGAACCCACCGATTTGACTGTTTGCCACC AAGTTTACATCATCACTTGCAGCAGCAACATCAACATTTAATGCAACAACAGGCTGCCTTGGCT GCGGCCCAACATCATCAGCAAATGCAGCACAATGCCGTTGTGGCCATGCATCAGGAACAGTTGC GTCGTGAGGTAGCCCAGGCTCAGG

$>$ Md-wg-3'-UTR (654bp) in PCR ${ }^{\circledR} I$

GGTGGTCGTGGTGGAGCGTTGTGCCTGTACATTCCACTGGTGCTGTGAAGTCAAGTGTA AATTGTGTCGAACGAAAAAGACGATACACACGTGTCTGTAAGGCCATGTCAAAGCCGAACGAT GTCGAGAAGAGACCTTGTGTCCAAGAGCGCAAGGCTTGTTATTTGTTGTAGTGTTTATTATTAT ATTTTTTTATAAAATATTCTCCTCCTCCTCCTCCTTGCCCCGTATCAATAATTCCCCCAGCTTGCC ATCCCCACTCCCTCCCGCCTGCTTAGATCGTCGACATACTTTGTAGTATTTAAAAATACAGGATT TGCTACGAATAAACTTTAGCGAAACAAAATGGAAATTGAAACCCACAAGAGTTGTTCGTTGGCT 
TGTCTTGTATTTGACAACAAATTAATTTGCCATACTTTGTAAATGTCACTGTAAATTAATAATAA ACCACATACGTACATACATTCACGTGTGCGTGTACGTGTGTGGTCTACATACAAGCATCTTTATT TAAGAGAAACGCTTCATTAACTAAGCGCTGGTTCAAAATTATTTATTTTTTGCATTTAATTTTAA GTTTCATTGTTTAATTTTAAATTTTGTTTCTTTGTTTGCTTAAAACTCGAAAAAAAAAAAAAAAA AAAAAAAAAAAAA

\subsection{Vector maps}

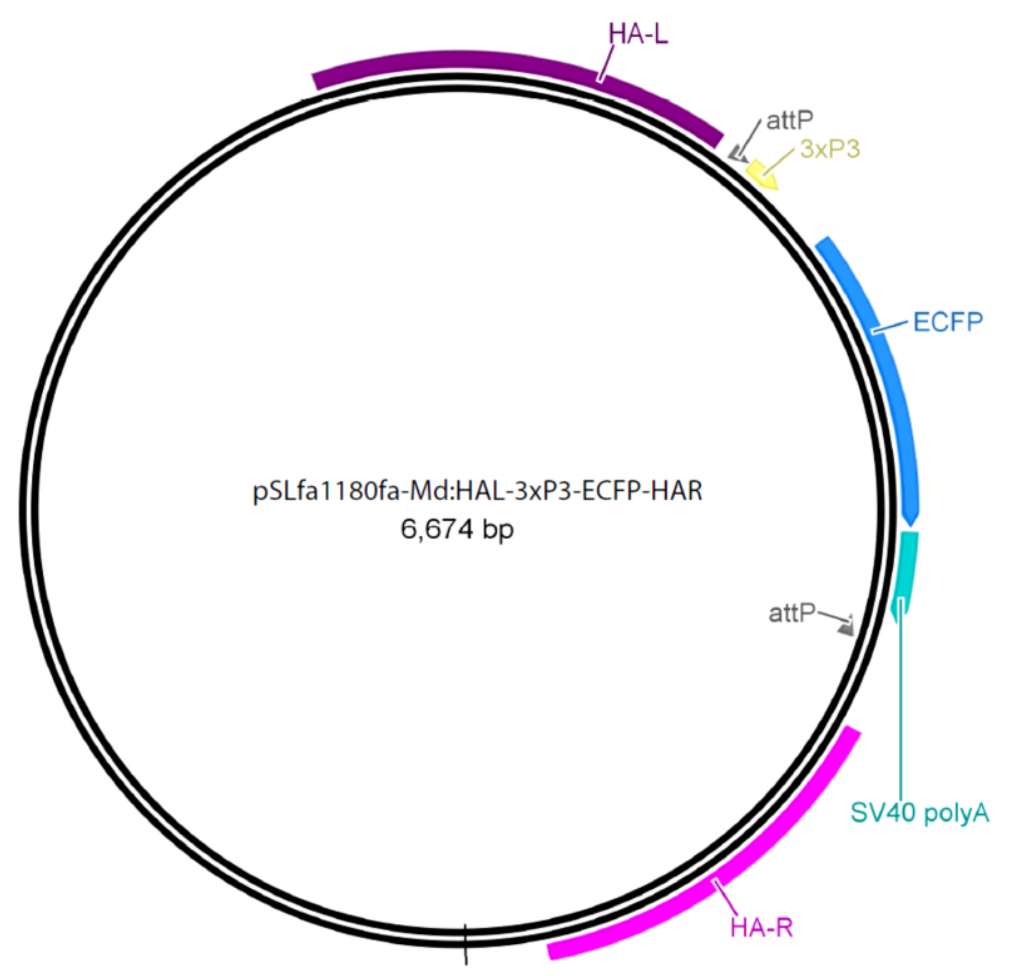

\section{$>$ pSLfa1180fa-Md:HAL-3xP3-ECFP-HAR}

TCTTCCGCTTCCTCGCTCACTGACTCGCTGCGCTCGGTCGTTCGGCTGCGGCGAGCGGTATCAG CTCACTCAAAGGCGGTAATACGGTTATCCACAGAATCAGGGGATAACGCAGGAAAGAACATGT GAGCAAAAGGCCAGCAAAAGGCCAGGAACCGTAAAAAGGCCGCGTTGCTGGCGTTTTTCCATA GGCTCCGCCCCCCTGACGAGCATCACAAAAATCGACGCTCAAGTCAGAGGTGGCGAAACCCGA CAGGACTATAAAGATACCAGGCGTTTCCCCCTGGAAGCTCCCTCGTGCGCTCTCCTGTTCCGAC CCTGCCGCTTACCGGATACCTGTCCGCCTTTCTCCCTTCGGGAAGCGTGGCGCTTTCTCATAGCT CACGCTGTAGGTATCTCAGTTCGGTGTAGGTCGTTCGCTCCAAGCTGGGCTGTGTGCACGAACC CCCCGTTCAGCCCGACCGCTGCGCCTTATCCGGTAACTATCGTCTTGAGTCCAACCCGGTAAGA CACGACTTATCGCCACTGGCAGCAGCCACTGGTAACAGGATTAGCAGAGCGAGGTATGTAGGC GGTGCTACAGAGTTCTTGAAGTGGTGGCCTAACTACGGCTACACTAGAAGAACAGTATTTGGTA TCTGCGCTCTGCTGAAGCCAGTTACCTTCGGAAAAAGAGTTGGTAGCTCTTGATCCGGCAAACA AACCACCGCTGGTAGCGGTGGTTTTTTTGTTTGCAAGCAGCAGATTACGCGCAGAAAAAAAGGA 
TCTCAAGAAGATCCTTTGATCTTTTCTACGGGGTCTGACGCTCAGTGGAACGAAAACTCACGTT AAGGGATTTTGGTCATGAGATTATCAAAAAGGATCTTCACCTAGATCCTTTTAAATTAAAAATG AAGTTTTAAATCAATCTAAAGTATATATGAGTAAACTTGGTCTGACAGTTACCAATGCTTAATCA GTGAGGCACCTATCTCAGCGATCTGTCTATTTCGTTCATCCATAGTTGCCTGACTCCCCGTCGTG TAGATAACTACGATACGGGAGGGCTTACCATCTGGCCCCAGTGCTGCAATGATACCGCGAGACC CACGCTCACCGGCTCCAGATTTATCAGCAATAAACCAGCCAGCCGGAAGGGCCGAGCGCAGAA GTGGTCCTGCAACTTTATCCGCCTCCATCCAGTCTATTAATTGTTGCCGGGAAGCTAGAGTAAG TAGTTCGCCAGTTAATAGTTTGCGCAACGTTGTTGCCATTGCTACAGGCATCGTGGTGTCACGC TCGTCGTTTGGTATGGCTTCATTCAGCTCCGGTTCCCAACGATCAAGGCGAGTTACATGATCCC CCATGTTGTGCAAAAAAGCGGTTAGCTCCTTCGGTCCTCCGATCGTTGTCAGAAGTAAGTTGGC CGCAGTGTTATCACTCATGGTTATGGCAGCACTGCATAATTCTCTTACTGTCATGCCATCCGTAA GATGCTTTTCTGTGACTGGTGAGTACTCAACCAAGTCATTCTGAGAATAGTGTATGCGGCGACC GAGTTGCTCTTGCCCGGCGTCAATACGGGATAATACCGCGCCACATAGCAGAACTTTAAAAGTG CTCATCATTGGAAAACGTTCTTCGGGGCGAAAACTCTCAAGGATCTTACCGCTGTTGAGATCCA GTTCGATGTAACCCACTCGTGCACCCAACTGATCTTCAGCATCTTTTACTTTCACCAGCGTTTCT GGGTGAGCAAAAACAGGAAGGCAAAATGCCGCAAAAAAGGGAATAAGGGCGACACGGAAATG TTGAATACTCATACTCTTCCTTTTTCAATATTATTGAAGCATTTATCAGGGTTATTGTCTCATGA GCGGATACATATTTGAATGTATTTAGAAAAATAAACAAATAGGGGTTCCGCGCACATTTCCCCG AAAAGTGCCACCTGACGTCTAAGAAACCATTATTATCATGACATTAACCTATAAAAATAGGCGT ATCACGAGGCCCTTTCGTCTCGCGCGTTTCGGTGATGACGGTGAAAACCTCTGACACATGCAGC TCCCGGAGACGGTCACAGCTTGTCTGTAAGCGGATGCCGGGAGCAGACAAGCCCGTCAGGGCG CGTCAGCGGGTGTTGGCGGGTGTCGGGGCTGGCTTAACTATGCGGCATCAGAGCAGATTGTACT GAGAGTGCACCATAAAATTGTAAACGTTAATATTTTGTTAAAATTCGCGTTAAATTTTTGTTAAA TCAGCTCATTTTTTAACCAATAGGCCGAAATCGGCAAAATCCCTTATAAATCAAAAGAATAGCC CGAGATAGGGTTGAGTGTTGTTCCAGTTTGGAACAAGAGTCCACTATTAAAGAACGTGGACTCC AACGTCAAAGGGCGAAAAACCGTCTATCAGGGCGATGGCCCACTACGTGAACCATCACCCAAA TCAAGTTTTTTGGGGTCGAGGTGCCGTAAAGCACTAAATCGGAACCCTAAAGGGAGCCCCCGAT TTAGAGCTTGACGGGGAAAGCCGGCGAACGTGGCGAGAAAGGAAGGGAAGAAAGCGAAAGGA GCGGGCGCTAGGGCGCTGGCAAGTGTAGCGGTCACGCTGCGCGTAACCACCACACCCGCCGCG CTTAATGCGCCGCTACAGGGCGCGTACTATGGTTGCTTTGACGTATGCGGTGTGAAATACCGCA CAGATGCGTAAGGAGAAAATACCGCATCAGGCGCCATTCGCCATTCAGGCTGCGCAACTGTTGG GAAGGGCGATCGGTGCGGGCCTCTTCGCTATTACGCCAGCTGGCGAAAGGGGGATGTGCTGCA AGGCGATTAAGTTGGGTAACGCCAGGGTTTTCCCAGTCACGACGTTGTAAAACGACGGCCAGTG CCAAGCTGGCCGGCCTAGGCGCGCCAAGCTTAAGGTGCACGGCCCACGTGGCCACTAGTACTT 
CTCGAGCTCTGTACATGTCCGCGGTCGCGACGTACGCGTATCGATGGCGCCAGCTGCAGGCGGC CGCCATATGCATCCTAGGCATATGCATCCTAGGCCTACTTAAAATCACCCACCCTAATCTCTATA TCTTTTTAAAACCCTCAAAGTGATCTGATTTTTTACTAATAACATCCGCTGTCTCTTCTTTCTCTO TTTGCAGATAGTAAAATCGACGATCGGTTTCCTCACCATCATCGGTTTCGTTTACATTTCGACGT GCGCAGCATACCCGCCGAGGAGAAACTGAAGGCTGCCGAACTGCAGCTGACACGTGAGGCCAT CAGTCATGCCACCCTCAATCCCCGCCTGGCCAATCGCACCCGCTACCAAGTGCTGGTCTATGAO ATAACACGGGTGGGTGTGCGCGGCAAGCGGGAGCCCAGCTATCTGCTGTTGGACAACAAGACO ATACGGCTGAATAGCACAGAGACGGTGAGTTTGGATGTCCAACCTGCTGTGGATCGGTGGCTG GCTTCACCGAAGAAGAATTTCGGTCTATTGGTGGAGGTGCGGACGTCACGCTCCCTGAAACCGG CACCGCATCATCATGTACGCTTGCGCCGCAGTGCGGACGAGGAACATGACGCGTGGCAGCGCA AACAGCCCCTGCTTTTTACATACACCGACGATGGACGGCACAAATCGCGTTCCATACGCGATGT CAGCAATCGTTCGAAGCGAGCTGGCCACAATCGTCGTTCGCATCGGCGAAAGAATAACGAGGA GATCTGTCGACGCCATTCGCTCTATGTGGACTTTACGGATGTCGGGTGGAGTGATTGGATTGTG GCGCCGCCGGGCTATGATGCCTTCTATTGTCACGGCAAATGTCCATTTCCGCTAGCGGAACATT TAAATTCGACAAATCATGCAGTGGTCCAGAATATGGTGAACAGCATTAATCCGGGGAAGGTACO AAAGGCGTGCTGTGTGCCAACACAGTTGGAGGGCATATCGATGCTCTATTTGAATGATCAGCGT ACGGTTGTGCTCAAGAATTATCAGGATATGACAGTGGTGGGGTTGTGGTTGTCGGCCTATTAAT ATTCCGGAGTATACGTACCGGCAACGTTGTAGTGCCCCAACTGGGGTAACCTTTGAGTTCTCTC AGTTGGGGGCGTAGGGGGGATTATTCATTAGAGACTAATTCAATTAGAGCTAATTCAATTAGGA TCCAAGCTTATCGATTTCGAACCCTCGACCGCCGGAGTATAAATAGAGGCGCTTCGTCTACGGA GCGACAATTCAATTCAAACAAGCAAAGTGAACACGTCGCTAAGCGAAAGCTAAGCAAATAAAC AAGCGCAGCTGAACAAGCTAAACAATCGGGGTACCGCTAGAGTCGACGGTACGATCCACCGGT CGCCACCATGGTGAGCAAGGGCGAGGAGCTGTTCACCGGGGTGGTGCCCATCCTGGTCGAGCT GGACGGCGACGTAAACGGCCACAAGTTCAGCGTGTCCGGCGAGGGCGAGGGCGATGCCACCTA CGGCAAGCTGACCCTGAAGTTCATCTGCACCACCGGCAAGCTGCCCGTGCCCTGGCCCACCCTO GTGACCACCCTGACCTGGGGCGTGCAGTGCTTCAGCCGCTACCCCGACCACATGAAGCAGCAC GACTTCTTCAAGTCCGCCATGCCCGAAGGCTACGTCCAGGAGCGCACCATCTTCTTCAAGGACG ACGGCAACTACAAGACCCGCGCCGAGGTGAAGTTCGAGGGCGACACCCTGGTGAACCGCATCG AGCTGAAGGGCATCGACTTCAAGGAGGACGGCAACATCCTGGGGCACAAGCTGGAGTACAACT ACATCAGCCACAACGTCTATATCACCGCCGACAAGCAGAAGAACGGCATCAAGGCCAACTTCA AGATCCGCCACAACATCGAGGACGGCAGCGTGCAGCTCGCCGACCACTACCAGCAGAACACCO CCATCGGCGACGGCCCCGTGCTGCTGCCCGACAACCACTACCTGAGCACCCAGTCCGCCCTGAG CAAAGACCCCAACGAGAAGCGCGATCACATGGTCCTGCTGGAGTTCGTGACCGCCGCCGGGAT CACTCTCGGCATGGACGAGCTGTACAAGTAAAGCGGCCGCGACTCTAGATCATAATCAGCCATA 
CCACATTTGTAGAGGTTTTACTTGCTTTAAAAAACCTCCCACACCTCCCCCTGAACCTGAAACAT AAAATGAATGCAATTGTTGTTGTTAACTTGTTTATTGCAGCTTATAATGGTTACAAATAAAGCAA TAGCATCACAAATTTCACAAATAAAGCATTTTTTTCACTGCATTCTAGTTGTGGTTTGTCCAAAC TCATCATCCCTACGCCCCCAACTGAGAGAACTCAAAGGTTACCCCAGTTGGGGCACTACAACAA CCGGTACCTCTAGAACTATAGCTAGCATGCGCAAATTTAAAGCGCTGATATCGATCGCGCGCAG ATCTGTCATGATGATCATTGCAATTGGATCCATATATAGGGCCCGGGTTATAATTACCTCAGGT CGACGTCCCATGGCCATTCGAATTCGGCCGGCCTAGGCGCGCCAATTCGTAATCATGGTCATAG CTGTTTCCTGTGTGTGAGGTTCGAGAGAGGAGAGAAGGACAACAACCACACAAAATAACTGAG

AAAAAAAAACGTGAGAAAAAGGGGGAGGCGAGAGGGCATACTTTTAGGGAGAGGGAGAGAGA AAAAGAGAGAGTGAGTTGGTGTAAAAATGCTTGGGCGCCAAATTGGTTTGGGAGATGAAAATG ACTTATATTTTCATAACTCCCCTGCCAAAGAAGAGTTATCCTTGGCCCTTATTTTTTCCACACCT TTGCACCAATGGAATACCCCCCTTTCCCCTTGGAATTTTTGTTCTTCTTTTGCCTCCCTTTTTGGC AACATATCGTGATATTTTCCTGCAGCCAGCTTTTTGGTTAAAGCTTAAAGCTGCATGCAACCACA ACAACAACAACGAAAAAGCAAGCAATAGAGCATCATTTGCTAGAATTGTAAAAGAAATTTGAG AGAGACAGAGAGAGAAAGAAACGCCCGCCCTTTGCTTGGCTGGCGATCTTAGCTTTGTCTTGGC TAAGTCTAGACTTTCTCTGGATCTGTATTTTTTTTTTTTGGTCGTTCTTTCTTTGCTTTTTAACGC TTTAATAAGTTTAAAGATTTTGTTTTTGGTAAATCAAGAAAAAAATATTATTATATATTAAAACA AAACAAACGCAATCTAAATGGACTGAATTATTGAATTTTATTTCTTAAATAAGTGTAAATATTAA ATATTAAAAAAAATAAATAAATAAAAAAAAACCGAAATGCTAGGGTTGACAACGAGGTGATAT ATAGATGTGAGGTGTATAAATGCAAAAATATCAATTCCCCTACAGGTCATAAGTAGTCACTAAA TGAGGAACTTTTCTTAAATAAAAAATAAATTAAAAAACACAAGCCGAACTAAAAACGAACAAAT TACATCCCCCCTCCCACACACTTGACAGCTAGGCGCTCACAATTCCACACAACATACGAGCCGG AAGCATAAAGTGTAAAGCCTGGGGTGCCTAATGAGTGAGCTAACTCACATTAATTGCGTTGCGC TCACTGCCCGCTTTCCAGTCGGGAAACCTGTCGTGCCAGCTGCATTAATGAATCGGCCAACGCG CGGGGAGAGGCGGTTTGCGTATTGGGCGC 


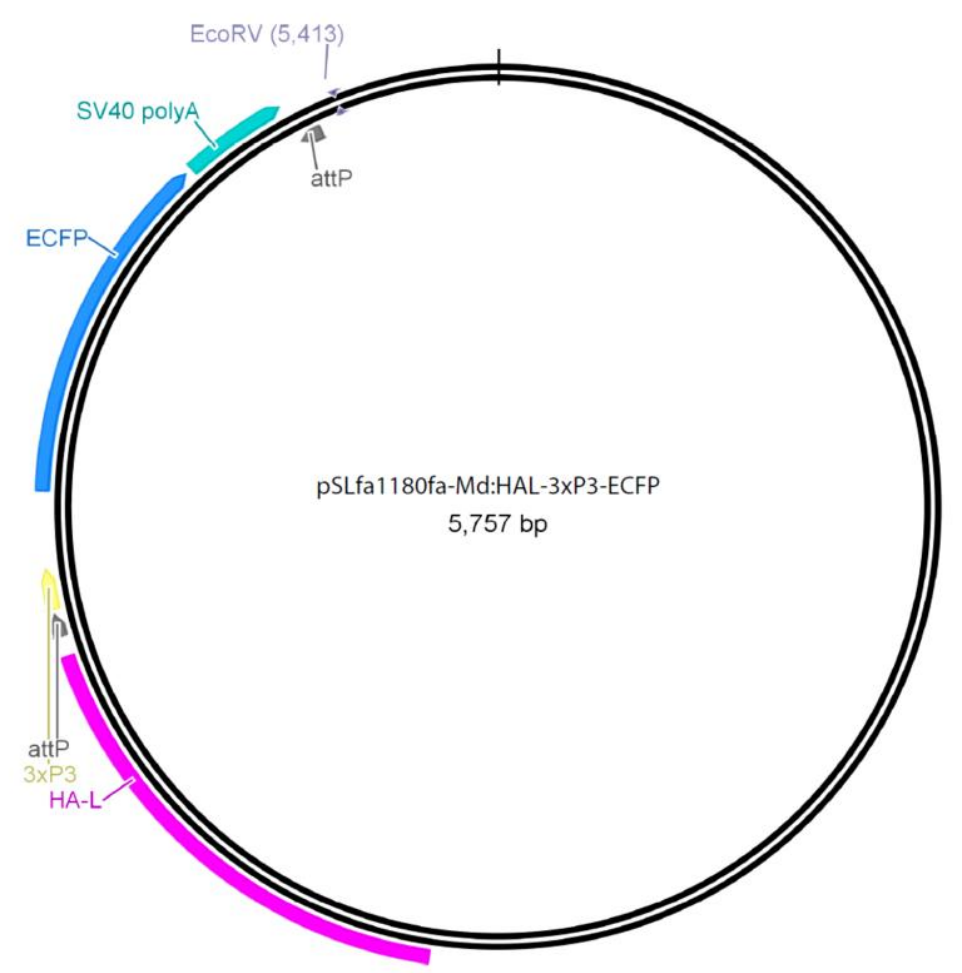

\section{$>$ pSLfa1180fa-Md:HAL-3xP3-ECFP}

TCTTCCGCTTCCTCGCTCACTGACTCGCTGCGCTCGGTCGTTCGGCTGCGGCGAGCGGTATCAG CTCACTCAAAGGCGGTAATACGGTTATCCACAGAATCAGGGGATAACGCAGGAAAGAACATGT GAGCAAAAGGCCAGCAAAAGGCCAGGAACCGTAAAAAGGCCGCGTTGCTGGCGTTTTTCCATA GGCTCCGCCCCCCTGACGAGCATCACAAAAATCGACGCTCAAGTCAGAGGTGGCGAAACCCGA CAGGACTATAAAGATACCAGGCGTTTCCCCCTGGAAGCTCCCTCGTGCGCTCTCCTGTTCCGAC CCTGCCGCTTACCGGATACCTGTCCGCCTTTCTCCCTTCGGGAAGCGTGGCGCTTTCTCATAGCT CACGCTGTAGGTATCTCAGTTCGGTGTAGGTCGTTCGCTCCAAGCTGGGCTGTGTGCACGAACC CCCCGTTCAGCCCGACCGCTGCGCCTTATCCGGTAACTATCGTCTTGAGTCCAACCCGGTAAGA CACGACTTATCGCCACTGGCAGCAGCCACTGGTAACAGGATTAGCAGAGCGAGGTATGTAGGC GGTGCTACAGAGTTCTTGAAGTGGTGGCCTAACTACGGCTACACTAGAAGAACAGTATTTGGTA TCTGCGCTCTGCTGAAGCCAGTTACCTTCGGAAAAAGAGTTGGTAGCTCTTGATCCGGCAAACA AACCACCGCTGGTAGCGGTGGTTTTTTTGTTTGCAAGCAGCAGATTACGCGCAGAAAAAAAGGA TCTCAAGAAGATCCTTTGATCTTTTCTACGGGGTCTGACGCTCAGTGGAACGAAAACTCACGTT AAGGGATTTTGGTCATGAGATTATCAAAAAGGATCTTCACCTAGATCCTTTTAAATTAAAAATG AAGTTTTAAATCAATCTAAAGTATATATGAGTAAACTTGGTCTGACAGTTACCAATGCTTAATCA GTGAGGCACCTATCTCAGCGATCTGTCTATTTCGTTCATCCATAGTTGCCTGACTCCCCGTCGTG TAGATAACTACGATACGGGAGGGCTTACCATCTGGCCCCAGTGCTGCAATGATACCGCGAGACC CACGCTCACCGGCTCCAGATTTATCAGCAATAAACCAGCCAGCCGGAAGGGCCGAGCGCAGAA GTGGTCCTGCAACTTTATCCGCCTCCATCCAGTCTATTAATTGTTGCCGGGAAGCTAGAGTAAG 
TAGTTCGCCAGTTAATAGTTTGCGCAACGTTGTTGCCATTGCTACAGGCATCGTGGTGTCACGC TCGTCGTTTGGTATGGCTTCATTCAGCTCCGGTTCCCAACGATCAAGGCGAGTTACATGATCCC CCATGTTGTGCAAAAAAGCGGTTAGCTCCTTCGGTCCTCCGATCGTTGTCAGAAGTAAGTTGGC CGCAGTGTTATCACTCATGGTTATGGCAGCACTGCATAATTCTCTTACTGTCATGCCATCCGTAA GATGCTTTTCTGTGACTGGTGAGTACTCAACCAAGTCATTCTGAGAATAGTGTATGCGGCGACC GAGTTGCTCTTGCCCGGCGTCAATACGGGATAATACCGCGCCACATAGCAGAACTTTAAAAGTG CTCATCATTGGAAAACGTTCTTCGGGGCGAAAACTCTCAAGGATCTTACCGCTGTTGAGATCCA GTTCGATGTAACCCACTCGTGCACCCAACTGATCTTCAGCATCTTTTACTTTCACCAGCGTTTCT GGGTGAGCAAAAACAGGAAGGCAAAATGCCGCAAAAAAGGGAATAAGGGCGACACGGAAATG TTGAATACTCATACTCTTCCTTTTTCAATATTATTGAAGCATTTATCAGGGTTATTGTCTCATGA GCGGATACATATTTGAATGTATTTAGAAAAATAAACAAATAGGGGTTCCGCGCACATTTCCCCG AAAAGTGCCACCTGACGTCTAAGAAACCATTATTATCATGACATTAACCTATAAAAATAGGCGT ATCACGAGGCCCTTTCGTCTCGCGCGTTTCGGTGATGACGGTGAAAACCTCTGACACATGCAGC TCCCGGAGACGGTCACAGCTTGTCTGTAAGCGGATGCCGGGAGCAGACAAGCCCGTCAGGGCG CGTCAGCGGGTGTTGGCGGGTGTCGGGGCTGGCTTAACTATGCGGCATCAGAGCAGATTGTACT GAGAGTGCACCATAAAATTGTAAACGTTAATATTTTGTTAAAATTCGCGTTAAATTTTTGTTAAA TCAGCTCATTTTTTAACCAATAGGCCGAAATCGGCAAAATCCCTTATAAATCAAAAGAATAGCC CGAGATAGGGTTGAGTGTTGTTCCAGTTTGGAACAAGAGTCCACTATTAAAGAACGTGGACTCC AACGTCAAAGGGCGAAAAACCGTCTATCAGGGCGATGGCCCACTACGTGAACCATCACCCAAA TCAAGTTTTTTGGGGTCGAGGTGCCGTAAAGCACTAAATCGGAACCCTAAAGGGAGCCCCCGAT TTAGAGCTTGACGGGGAAAGCCGGCGAACGTGGCGAGAAAGGAAGGGAAGAAAGCGAAAGGA GCGGGCGCTAGGGCGCTGGCAAGTGTAGCGGTCACGCTGCGCGTAACCACCACACCCGCCGCG CTTAATGCGCCGCTACAGGGCGCGTACTATGGTTGCTTTGACGTATGCGGTGTGAAATACCGCA CAGATGCGTAAGGAGAAAATACCGCATCAGGCGCCATTCGCCATTCAGGCTGCGCAACTGTTGG GAAGGGCGATCGGTGCGGGCCTCTTCGCTATTACGCCAGCTGGCGAAAGGGGGATGTGCTGCA AGGCGATTAAGTTGGGTAACGCCAGGGTTTTCCCAGTCACGACGTTGTAAAACGACGGCCAGTG CCAAGCTGGCCGGCCTAGGCGCGCCAAGCTTAAGGTGCACGGCCCACGTGGCCACTAGTACTT CTCGAGCTCTGTACATGTCCGCGGTCGCGACGTACGCGTATCGATGGCGCCAGCTGCAGGCGGC CGCCATATGCATCCTAGGCATATGCATCCTAGGCCTACTTAAAATCACCCACCCTAATCTCTATA TCTTTTTAAAACCCTCAAAGTGATCTGATTTTTTACTAATAACATCCGCTGTCTCTTCTTTCTCTO TTTGCAGATAGTAAAATCGACGATCGGTTTCCTCACCATCATCGGTTTCGTTTACATTTCGACGT GCGCAGCATACCCGCCGAGGAGAAACTGAAGGCTGCCGAACTGCAGCTGACACGTGAGGCCAT CAGTCATGCCACCCTCAATCCCCGCCTGGCCAATCGCACCCGCTACCAAGTGCTGGTCTATGAO ATAACACGGGTGGGTGTGCGCGGCAAGCGGGAGCCCAGCTATCTGCTGTTGGACAACAAGACC 
ATACGGCTGAATAGCACAGAGACGGTGAGTTTGGATGTCCAACCTGCTGTGGATCGGTGGCTG GCTTCACCGAAGAAGAATTTCGGTCTATTGGTGGAGGTGCGGACGTCACGCTCCCTGAAACCGG CACCGCATCATCATGTACGCTTGCGCCGCAGTGCGGACGAGGAACATGACGCGTGGCAGCGCA AACAGCCCCTGCTTTTTACATACACCGACGATGGACGGCACAAATCGCGTTCCATACGCGATGT CAGCAATCGTTCGAAGCGAGCTGGCCACAATCGTCGTTCGCATCGGCGAAAGAATAACGAGGA GATCTGTCGACGCCATTCGCTCTATGTGGACTTTACGGATGTCGGGTGGAGTGATTGGATTGTG GCGCCGCCGGGCTATGATGCCTTCTATTGTCACGGCAAATGTCCATTTCCGCTAGCGGAACATT TAAATTCGACAAATCATGCAGTGGTCCAGAATATGGTGAACAGCATTAATCCGGGGAAGGTACO AAAGGCGTGCTGTGTGCCAACACAGTTGGAGGGCATATCGATGCTCTATTTGAATGATCAGCGT ACGGTTGTGCTCAAGAATTATCAGGATATGACAGTGGTGGGGTTGTGGTTGTCGGCCTATTAAT ATTCCGGAGTATACGTACCGGCAACGTTGTAGTGCCCCAACTGGGGTAACCTTTGAGTTCTCTC AGTTGGGGGCGTAGGGGGGATTATTCATTAGAGACTAATTCAATTAGAGCTAATTCAATTAGGA TCCAAGCTTATCGATTTCGAACCCTCGACCGCCGGAGTATAAATAGAGGCGCTTCGTCTACGGA GCGACAATTCAATTCAAACAAGCAAAGTGAACACGTCGCTAAGCGAAAGCTAAGCAAATAAAC AAGCGCAGCTGAACAAGCTAAACAATCGGGGTACCGCTAGAGTCGACGGTACGATCCACCGGT CGCCACDATGGTGAGCAAGGGCGAGGAGCTGTTCACCGGGGTGGTGCCCATCCTGGTCGAGCT GGACGGCGACGTAAACGGCCACAAGTTCAGCGTGTCCGGCGAGGGCGAGGGCGATGCCACCTA CGGCAAGCTGACCCTGAAGTTCATCTGCACCACCGGCAAGCTGCCCGTGCCCTGGCCCACCCTO GTGACCACCCTGACCTGGGGCGTGCAGTGCTTCAGCCGCTACCCCGACCACATGAAGCAGCAC GACTTCTTCAAGTCCGCCATGCCCGAAGGCTACGTCCAGGAGCGCACCATCTTCTTCAAGGACG ACGGCAACTACAAGACCCGCGCCGAGGTGAAGTTCGAGGGCGACACCCTGGTGAACCGCATCG AGCTGAAGGGCATCGACTTCAAGGAGGACGGCAACATCCTGGGGCACAAGCTGGAGTACAACT ACATCAGCCACAACGTCTATATCACCGCCGACAAGCAGAAGAACGGCATCAAGGCCAACTTCA AGATCCGCCACAACATCGAGGACGGCAGCGTGCAGCTCGCCGACCACTACCAGCAGAACACCO CCATCGGCGACGGCCCCGTGCTGCTGCCCGACAACCACTACCTGAGCACCCAGTCCGCCCTGAG CAAAGACCCCAACGAGAAGCGCGATCACATGGTCCTGCTGGAGTTCGTGACCGCCGCCGGGAT CACTCTCGGCATGGACGAGCTGTACAAGTAAAGCGGCCGCGACTCTAGATCATAATCAGCCATA CCACATTTGTAGAGGTTTTACTTGCTTTAAAAAACCTCCCACACCTCCCCCTGAACCTGAAACAT AAAATGAATGCAATTGTTGTTGTTAACTTGTTTATTGCAGCTTATAATGGTTACAAATAAAGCAA TAGCATCACAAATTTCACAAATAAAGCATTTTTTTCACTGCATTCTAGTTGTGGTTTGTCCAAAC TCATCATCCCTACGCCCCCAACTGAGAGAACTCAAAGGTTACCCCAGTTGGGGCACTACAACAA CCGGTACCTCTAGAACTATAGCTAGCATGCGCAAATTTAAAGCGCTGATATCGATCGCGCGCAG ATCTGTCATGATGATCATTGCAATTGGATCCATATATAGGGCCCGGGTTATAATTACCTCAGGT CGACGTCCCATGGCCATTCGAATTCGGCCGGCCTAGGCGCGCCAATTCGTAATCATGGTCATAG 
CTGTTTCCTGTGTGCGCTCACAATTCCACACAACATACGAGCCGGAAGCATAAAGTGTAAAGCC TGGGGTGCCTAATGAGTGAGCTAACTCACATTAATTGCGTTGCGCTCACTGCCCGCTTTCCAGT CGGGAAACCTGTCGTGCCAGCTGCATTAATGAATCGGCCAACGCGCGGGGAGAGGCGGTTTGC GTATTGGGCGC

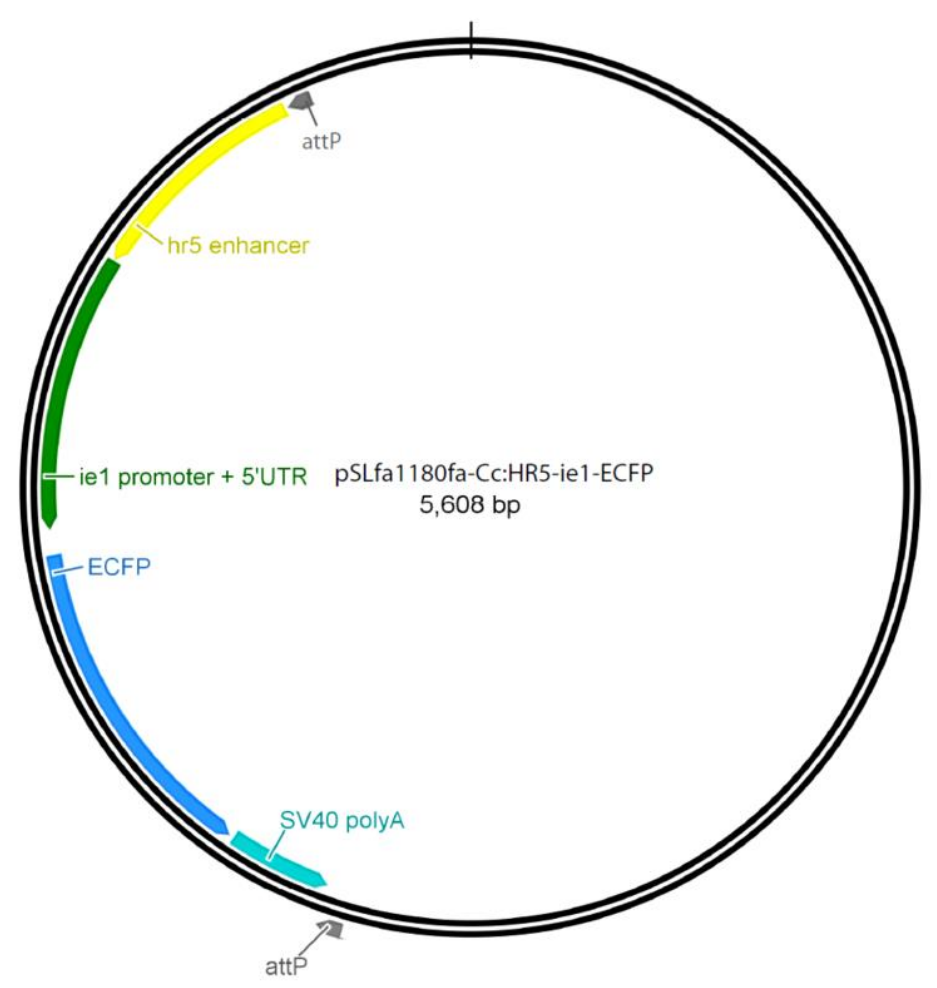

$>$ pSLfa1180fa-Cc:HR5-ie1-ECFP

TCTTCCGCTTCCTCGCTCACTGACTCGCTGCGCTCGGTCGTTCGGCTGCGGCGAGCGGTATCAG CTCACTCAAAGGCGGTAATACGGTTATCCACAGAATCAGGGGATAACGCAGGAAAGAACATGT GAGCAAAAGGCCAGCAAAAGGCCAGGAACCGTAAAAAGGCCGCGTTGCTGGCGTTTTTCCATA GGCTCCGCCCCCCTGACGAGCATCACAAAAATCGACGCTCAAGTCAGAGGTGGCGAAACCCGA CAGGACTATAAAGATACCAGGCGTTTCCCCCTGGAAGCTCCCTCGTGCGCTCTCCTGTTCCGAC CCTGCCGCTTACCGGATACCTGTCCGCCTTTCTCCCTTCGGGAAGCGTGGCGCTTTCTCATAGCT CACGCTGTAGGTATCTCAGTTCGGTGTAGGTCGTTCGCTCCAAGCTGGGCTGTGTGCACGAACC CCCCGTTCAGCCCGACCGCTGCGCCTTATCCGGTAACTATCGTCTTGAGTCCAACCCGGTAAGA CACGACTTATCGCCACTGGCAGCAGCCACTGGTAACAGGATTAGCAGAGCGAGGTATGTAGGC GGTGCTACAGAGTTCTTGAAGTGGTGGCCTAACTACGGCTACACTAGAAGAACAGTATTTGGTA TCTGCGCTCTGCTGAAGCCAGTTACCTTCGGAAAAAGAGTTGGTAGCTCTTGATCCGGCAAACA AACCACCGCTGGTAGCGGTGGTTTTTTTGTTTGCAAGCAGCAGATTACGCGCAGAAAAAAAGGA TCTCAAGAAGATCCTTTGATCTTTTCTACGGGGTCTGACGCTCAGTGGAACGAAAACTCACGTT 
AAGGGATTTTGGTCATGAGATTATCAAAAAGGATCTTCACCTAGATCCTTTTAAATTAAAAATG AAGTTTTAAATCAATCTAAAGTATATATGAGTAAACTTGGTCTGACAGTTACCAATGCTTAATCA GTGAGGCACCTATCTCAGCGATCTGTCTATTTCGTTCATCCATAGTTGCCTGACTCCCCGTCGTG TAGATAACTACGATACGGGAGGGCTTACCATCTGGCCCCAGTGCTGCAATGATACCGCGAGACC CACGCTCACCGGCTCCAGATTTATCAGCAATAAACCAGCCAGCCGGAAGGGCCGAGCGCAGAA GTGGTCCTGCAACTTTATCCGCCTCCATCCAGTCTATTAATTGTTGCCGGGAAGCTAGAGTAAG TAGTTCGCCAGTTAATAGTTTGCGCAACGTTGTTGCCATTGCTACAGGCATCGTGGTGTCACGC TCGTCGTTTGGTATGGCTTCATTCAGCTCCGGTTCCCAACGATCAAGGCGAGTTACATGATCCC CCATGTTGTGCAAAAAAGCGGTTAGCTCCTTCGGTCCTCCGATCGTTGTCAGAAGTAAGTTGGC CGCAGTGTTATCACTCATGGTTATGGCAGCACTGCATAATTCTCTTACTGTCATGCCATCCGTAA GATGCTTTTCTGTGACTGGTGAGTACTCAACCAAGTCATTCTGAGAATAGTGTATGCGGCGACC GAGTTGCTCTTGCCCGGCGTCAATACGGGATAATACCGCGCCACATAGCAGAACTTTAAAAGTG CTCATCATTGGAAAACGTTCTTCGGGGCGAAAACTCTCAAGGATCTTACCGCTGTTGAGATCCA GTTCGATGTAACCCACTCGTGCACCCAACTGATCTTCAGCATCTTTTACTTTCACCAGCGTTTCT GGGTGAGCAAAAACAGGAAGGCAAAATGCCGCAAAAAAGGGAATAAGGGCGACACGGAAATG TTGAATACTCATACTCTTCCTTTTTCAATATTATTGAAGCATTTATCAGGGTTATTGTCTCATGA GCGGATACATATTTGAATGTATTTAGAAAAATAAACAAATAGGGGTTCCGCGCACATTTCCCCG AAAAGTGCCACCTGACGTCTAAGAAACCATTATTATCATGACATTAACCTATAAAAATAGGCGT ATCACGAGGCCCTTTCGTCTCGCGCGTTTCGGTGATGACGGTGAAAACCTCTGACACATGCAGC TCCCGGAGACGGTCACAGCTTGTCTGTAAGCGGATGCCGGGAGCAGACAAGCCCGTCAGGGCG CGTCAGCGGGTGTTGGCGGGTGTCGGGGCTGGCTTAACTATGCGGCATCAGAGCAGATTGTACT GAGAGTGCACCATAAAATTGTAAACGTTAATATTTTGTTAAAATTCGCGTTAAATTTTTGTTAAA TCAGCTCATTTTTTAACCAATAGGCCGAAATCGGCAAAATCCCTTATAAATCAAAAGAATAGCC CGAGATAGGGTTGAGTGTTGTTCCAGTTTGGAACAAGAGTCCACTATTAAAGAACGTGGACTCC AACGTCAAAGGGCGAAAAACCGTCTATCAGGGCGATGGCCCACTACGTGAACCATCACCCAAA TCAAGTTTTTTGGGGTCGAGGTGCCGTAAAGCACTAAATCGGAACCCTAAAGGGAGCCCCCGAT TTAGAGCTTGACGGGGAAAGCCGGCGAACGTGGCGAGAAAGGAAGGGAAGAAAGCGAAAGGA GCGGGCGCTAGGGCGCTGGCAAGTGTAGCGGTCACGCTGCGCGTAACCACCACACCCGCCGCG CTTAATGCGCCGCTACAGGGCGCGTACTATGGTTGCTTTGACGTATGCGGTGTGAAATACCGCA CAGATGCGTAAGGAGAAAATACCGCATCAGGCGCCATTCGCCATTCAGGCTGCGCAACTGTTGG GAAGGGCGATCGGTGCGGGCCTCTTCGCTATTACGCCAGCTGGCGAAAGGGGGATGTGCTGCA AGGCGATTAAGTTGGGTAACGCCAGGGTTTTCCCAGTCACGACGTTGTAAAACGACGGCCAGTG CCAAGCTGGCCGGCCTAGGCGCGCCAAGCTTAAGGTGCACGGCCCACGTGGCCACTAGTACTT CTCGAGCTCTGTACATGTCCGCGGTCGCGACGTACGCGTATCGATGGCGCCAGCTGCAGGCGGC 
CGCCATATGCATCCTAGGCCTATTAATATTCCGGAGTATACGTAGCCGGCTAACGTTGTAGTGC CCCAACTGGGGTAACCTTTGAGTTCTCTCAGTTGGGGGCGTAGGGATGATGAGTTTGGACAAAC CACAACTAGAATGCAGTGAAAAAAATGCTTTATTTGTGAAATTTGTGATGCTATTGCTTTATTTG TAACCATTATAAGCTGCAATAAACAAGTTAACAACAACAATTGCATTCATTTTATGTTTCAGGTT CAGGGGGAGGTGTGGGAGGTTTTTTAAAGCAAGTAAAACCTCTACAAATGTGGTATGGCTGATT ATGATCTAGAGTCGCGGCCGCTTTACTTGTACAGCTCGTCCATGCCGAGAGTGATCCCGGCGGO GGTCACGAACTCCAGCAGGACCATGTGATCGCGCTTCTCGTTGGGGTCTTTGCTCAGGGCGGAC TGGGTGCTCAGGTAGTGGTTGTCGGGCAGCAGCACGGGGCCGTCGCCGATGGGGGTGTTCTGC TGGTAGTGGTCGGCGAGCTGCACGCTGCCGTCCTCGATGTTGTGGCGGATCTTGAAGTTGGCCT TGATGCCGTTCTTCTGCTTGTCGGCGGTGATATAGACGTTGTGGCTGATGTAGTTGTACTCCAG CTTGTGCCCCAGGATGTTGCCGTCCTCCTTGAAGTCGATGCCCTTCAGCTCGATGCGGTTCACO AGGGTGTCGCCCTCGAACTTCACCTCGGCGCGGGTCTTGTAGTTGCCGTCGTCCTTGAAGAAGA TGGTGCGCTCCTGGACGTAGCCTTCGGGCATGGCGGACTTGAAGAAGTCGTGCTGCTTCATGTG GTCGGGGTAGCGGCTGAAGCACTGCACGCCCCAGGTCAGGGTGGTCACGAGGGTGGGCCAGGG CACGGGCAGCTTGCCGGTGGTGCAGATGAACTTCAGGGTCAGCTTGCCGTAGGTGGCATCGCO CTCGCCCTCGCCGGACACGCTGAACTTGTGGCCGTTTACGTCGCCGTCCAGCTCGACCAGGATG GGCACCACCCCGGTGAACAGCTCCTCGCCCTTGCTCACCATAACAACCGGTACCTCTAGAACTA TAGCTAGCATGCGCAAATTTAAAGCGCTGATGTCACTTGGTTGTTCACGATCTTGTCGCCGCCA GTGTCAACTTGCAACTGAAACAATATCCAACATGAACGTCAATTTATACTGCCCTAATGGCGAA CACGATAACAATATTTCTTTTATTATGCCCTCTAAAACCAACGCGGTTATCGTTTATTTATTCAA ATTAGATATAGAACATCCGCCGACATACAATGTTAATGCAAAAACGCGTTTGGTGAGCGGATAC GAAAACAGTCGGCCGATAAACATTAATCTGAGGTCGATAACACCGTCCTTGAACGGAACACGA GGAGCGTACGTGATCAGCTGCATTCGCGCGCCGCGCCTTTATCGAGATTTATTTGCATACAACA AGTACACTGCGCCGTTGGGATTTGTGGTAACGCGCACACATGCAGAGCTGCAAGTGTGGCACAT TTTGTCTGTGCGCAAAACCTTTGAAGCCAAAAGTACGAGGTCCGTTACGGGCATGCTAGCGCAC ACGGACAATGGACCCGACAAATTCTACGCCAAGGATTTAATGATAATGTCGGGCAACGTATCCG TTCATTTTATCAATAACCTACAAAAATGTCGCGCGCATCACAAAGACATCGACG

CGCGTAGAATTCTACCCGTAAAGCGAGTTTAGTTATGAGCCATGTGCAAAACATGACATCAGCT TTTATTTTTATAACAAATGACATCATTTCTTGATTGTGTTTTACACGTAGAATTCTACTCGTAAA GCCGAGAGTTCAGTTTTGAAAAACAAATGACATCATCTTTTTGATTGTGCTTTACGAGTAGAATT CTACCCGTAAATCAAGTTCGGTTTTGAAAAACAAATGAGTCATATTGTATGATATCATATTGCA AAACAAATGACTCATCAATCGATCGTGCGTTACACGTAGAATTCTACTCGTAAAGCAGTTTATG AGCCCGTGTGCAAAACATGACATCATCTCGATTTGAAAAACAAATGACCATCATCCACTCGATC GTGCGTTACAAGTAGAATTCTACTCGTAAAGCCAGTTCGGTTATGAGCCGTGTGCAAAACATGA 
CATCAGCTTATGACTCATACTTGATTGTGTTTTACGCGCCCATCCGACCCTACGCCCCCAACTGA GAGAACTCAAAGGTTACCCCAGTTGGGGCACCAGATCTGTCATGATGATCATTGCAATTGGATC CATATATAGGGCCCGGGTTATAATTACCTCAGGTCGACGTCCCATGGCCATTCGAATTCGGCCG GCCTAGGCGCGCCAATTCGTAATCATGGTCATAGCTGTTTCCTGTGTGAAATTGTTATCCGCTCA CAATTCCACACAACATACGAGCCGGAAGCATAAAGTGTAAAGCCTGGGGTGCCTAATGAGTGA GCTAACTCACATTAATTGCGTTGCGCTCACTGCCCGCTTTCCAGTCGGGAAACCTGTCGTGCCA GCTGCATTAATGAATCGGCCAACGCGCGGGGAGAGGCGGTTTGCGTATTGGGCGC

\section{$>$ pSLfa1180fa}

TCTTCCGCTTCCTCGCTCACTGACTCGCTGCGCTCGGTCGTTCGGCTGCGGCGAGCGGTATCAG CTCACTCAAAGGCGGTAATACGGTTATCCACAGAATCAGGGGATAACGCAGGAAAGAACATGT GAGCAAAAGGCCAGCAAAAGGCCAGGAACCGTAAAAAGGCCGCGTTGCTGGCGTTTTTCCATA GGCTCCGCCCCCCTGACGAGCATCACAAAAATCGACGCTCAAGTCAGAGGTGGCGAAACCCGA CAGGACTATAAAGATACCAGGCGTTTCCCCCTGGAAGCTCCCTCGTGCGCTCTCCTGTTCCGAC CCTGCCGCTTACCGGATACCTGTCCGCCTTTCTCCCTTCGGGAAGCGTGGCGCTTTCTCATAGCT CACGCTGTAGGTATCTCAGTTCGGTGTAGGTCGTTCGCTCCAAGCTGGGCTGTGTGCACGAACC CCCCGTTCAGCCCGACCGCTGCGCCTTATCCGGTAACTATCGTCTTGAGTCCAACCCGGTAAGA CACGACTTATCGCCACTGGCAGCAGCCACTGGTAACAGGATTAGCAGAGCGAGGTATGTAGGC GGTGCTACAGAGTTCTTGAAGTGGTGGCCTAACTACGGCTACACTAGAAGAACAGTATTTGGTA TCTGCGCTCTGCTGAAGCCAGTTACCTTCGGAAAAAGAGTTGGTAGCTCTTGATCCGGCAAACA AACCACCGCTGGTAGCGGTGGTTTTTTTGTTTGCAAGCAGCAGATTACGCGCAGAAAAAAAGGA TCTCAAGAAGATCCTTTGATCTTTTCTACGGGGTCTGACGCTCAGTGGAACGAAAACTCACGTT AAGGGATTTTGGTCATGAGATTATCAAAAAGGATCTTCACCTAGATCCTTTTAAATTAAAAATG AAGTTTTAAATCAATCTAAAGTATATATGAGTAAACTTGGTCTGACAGTTACCAATGCTTAATCA GTGAGGCACCTATCTCAGCGATCTGTCTATTTCGTTCATCCATAGTTGCCTGACTCCCCGTCGTG TAGATAACTACGATACGGGAGGGCTTACCATCTGGCCCCAGTGCTGCAATGATACCGCGAGACC CACGCTCACCGGCTCCAGATTTATCAGCAATAAACCAGCCAGCCGGAAGGGCCGAGCGCAGAA GTGGTCCTGCAACTTTATCCGCCTCCATCCAGTCTATTAATTGTTGCCGGGAAGCTAGAGTAAG TAGTTCGCCAGTTAATAGTTTGCGCAACGTTGTTGCCATTGCTACAGGCATCGTGGTGTCACGC TCGTCGTTTGGTATGGCTTCATTCAGCTCCGGTTCCCAACGATCAAGGCGAGTTACATGATCCC CCATGTTGTGCAAAAAAGCGGTTAGCTCCTTCGGTCCTCCGATCGTTGTCAGAAGTAAGTTGGC CGCAGTGTTATCACTCATGGTTATGGCAGCACTGCATAATTCTCTTACTGTCATGCCATCCGTAA GATGCTTTTCTGTGACTGGTGAGTACTCAACCAAGTCATTCTGAGAATAGTGTATGCGGCGACC GAGTTGCTCTTGCCCGGCGTCAATACGGGATAATACCGCGCCACATAGCAGAACTTTAAAAGTG 
CTCATCATTGGAAAACGTTCTTCGGGGCGAAAACTCTCAAGGATCTTACCGCTGTTGAGATCCA GTTCGATGTAACCCACTCGTGCACCCAACTGATCTTCAGCATCTTTTACTTTCACCAGCGTTTCT GGGTGAGCAAAAACAGGAAGGCAAAATGCCGCAAAAAAGGGAATAAGGGCGACACGGAAATG TTGAATACTCATACTCTTCCTTTTTCAATATTATTGAAGCATTTATCAGGGTTATTGTCTCATGA GCGGATACATATTTGAATGTATTTAGAAAAATAAACAAATAGGGGTTCCGCGCACATTTCCCCG AAAAGTGCCACCTGACGTCTAAGAAACCATTATTATCATGACATTAACCTATAAAAATAGGCGT ATCACGAGGCCCTTTCGTCTCGCGCGTTTCGGTGATGACGGTGAAAACCTCTGACACATGCAGC TCCCGGAGACGGTCACAGCTTGTCTGTAAGCGGATGCCGGGAGCAGACAAGCCCGTCAGGGCG CGTCAGCGGGTGTTGGCGGGTGTCGGGGCTGGCTTAACTATGCGGCATCAGAGCAGATTGTACT GAGAGTGCACCATAAAATTGTAAACGTTAATATTTTGTTAAAATTCGCGTTAAATTTTTGTTAAA TCAGCTCATTTTTTAACCAATAGGCCGAAATCGGCAAAATCCCTTATAAATCAAAAGAATAGCC CGAGATAGGGTTGAGTGTTGTTCCAGTTTGGAACAAGAGTCCACTATTAAAGAACGTGGACTCC AACGTCAAAGGGCGAAAAACCGTCTATCAGGGCGATGGCCCACTACGTGAACCATCACCCAAA TCAAGTTTTTTGGGGTCGAGGTGCCGTAAAGCACTAAATCGGAACCCTAAAGGGAGCCCCCGAT TTAGAGCTTGACGGGGAAAGCCGGCGAACGTGGCGAGAAAGGAAGGGAAGAAAGCGAAAGGA GCGGGCGCTAGGGCGCTGGCAAGTGTAGCGGTCACGCTGCGCGTAACCACCACACCCGCCGCG CTTAATGCGCCGCTACAGGGCGCGTACTATGGTTGCTTTGACGTATGCGGTGTGAAATACCGCA CAGATGCGTAAGGAGAAAATACCGCATCAGGCGCCATTCGCCATTCAGGCTGCGCAACTGTTGG GAAGGGCGATCGGTGCGGGCCTCTTCGCTATTACGCCAGCTGGCGAAAGGGGGATGTGCTGCA AGGCGATTAAGTTGGGTAACGCCAGGGTTTTCCCAGTCACGACGTTGTAAAACGACGGCCAGTG CCAAGCTGGCCGGCCTAGGCGCGCCAAGCTTAAGGTGCACGGCCCACGTGGCCACTAGTACTT CTCGAGCTCTGTACATGTCCGCGGTCGCGACGTACGCGTATCGATGGCGCCAGCTGCAGGCGGC CGCCATATGCATCCTAGGCCTATTAATATTCCGGAGTATACGTAGCCGGCTAACGTTAACAACC GGTACCTCTAGAACTATAGCTAGCATGCGCAAATTTAAAGCGCTGATATCGATCGCGCGCAGAT CTGTCATGATGATCATTGCAATTGGATCCATATATAGGGCCCGGGTTATAATTACCTCAGGTCG ACGTCCCATGGCCATTCGAATTCGGCCGGCCTAGGCGCGCCAATTCGTAATCATGGTCATAGCT GTTTCCTGTGTGAAATTGTTATCCGCTCACAATTCCACACAACATACGAGCCGGAAGCATAAAG TGTAAAGCCTGGGGTGCCTAATGAGTGAGCTAACTCACATTAATTGCGTTGCGCTCACTGCCCG CTTTCCAGTCGGGAAACCTGTCGTGCCAGCTGCATTAATGAATCGGCCAACGCGCGGGGAGAG GCGGTTTGCGTATTGGGCGC 


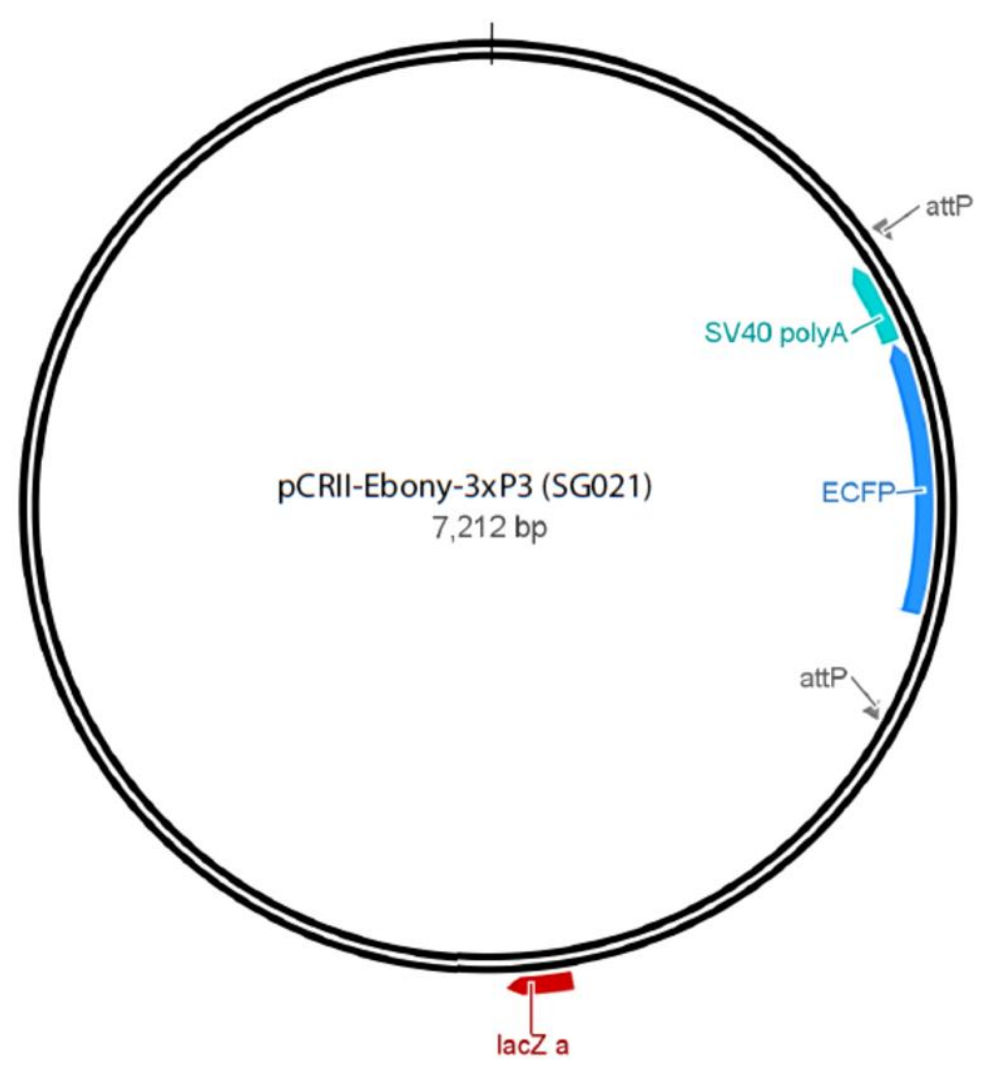

$>$ pCRII-Ebony-3xP3 (SG021)

GCTATGCATCAAGCTGGACAGCGACTGGTGCCTCTGGCAATTGGCTAGCTCCATGCAATTAACG AGTTAGCATGCCGCATTCCGAGCGCTTGTTTAGGCTTTTGGCCAAGTCCAATGTCCGAGATACA ACAGTTGAGCCAGCGTAACTCCTTCAAGATGAGGCATTTTATGGAGCTAGGTTATTCAAAACTC GAAAATTATTATTTTTATTTTTAAGGCACATTTTTTTAGGAATAAATACCTGGCAATGTAAACGT GGATTTTCAAAGCCGGAACACATCTCAATTCATAGTGTAAGCCCACATAGATTAAACTGACCGT AATTAGTAACGACAAGCAGGTGGCTATGCTAATGCATTAAGTAATATTTACTAAAGTTAAGGAG CTCTCTAGTCTCCTATTAGACAAACCTGTAATGAAAGTATGACTGATAGGTTAGGGAAAAATAT GGTGCTAAGCGAATAGATAAAAATGTCGCCATAATTTAAAATAACATGTGCCTGTTGCTAAGCG TTTAGTCGCAAAGAAGAAAAACTAACTTAACTATTACTGAAAATAAAATGAATGAGTCTTTATA CTCCACGCCATTTAGCGAGAAATAAGAATTCCAACTATTTCCATAAGGCTATTCTTAAGTACTG ATATAAATAATAAAACATTAATAAAATCGTATTACCCTGAAACCCTTAATTATCAAGTCATTAAC ATGGAATATCGAATTCCAGTTTACTTGAACTTGCAATCTCTGCAGCTTAAGAGACAGCAATTTA ATTGAATAAATATAGCTCAACAGAAGCCATCCGTTTAAGCCAGCTCCCATATTCATACTCATTCA TTATAATAGTTTATTGTGCGAGCGCTCCATTAACACGAAGTGTTATAAATATGCATGTATGAATG AATTTAATTGCTACCGCAAGGCAGCAAGGCATTAGCCTGCATTGCATAATACGAGCTGGGAATG ATGATGTAACCTAGGGATTCTCCGACTGAGATTCTAAGCCCAAAACTAACAAAGTATTCCCCAC AGTTAATATATCTTCAAGATGGGTTCGCTGCCACAATTGTCGATCGGAGTAGTGCCCCAACTGG 
GGTAACCTTTGAGTTCTCTCAGTTGGGGGCGTAGGGATGATGAGTTTGGACAAACCACAACTAG AATGCAGTGAAAAAAATGCTTTATTTGTGAAATTTGTGATGCTATTGCTTTATTTGTAACCATTA TAAGCTGCAATAAACAAGTTAACAACAACAATTGCATTCATTTTATGTTTCAGGTTCAGGGGGA GGTGTGGGAGGTTTTTTAAAGCAAGTAAAACCTCTACAAATGTGGTATGGCTGATTATGATCTA GAGTCGCGGCCGCTTTACTTGTACAGCTCGTCCATGCCGAGAGTGATCCCGGCGGCGGTCACGA ACTCCAGCAGGACCATGTGATCGCGCTTCTCGTTGGGGTCTTTGCTCAGGGCGGACTGGGTGCT CAGGTAGTGGTTGTCGGGCAGCAGCACGGGGCCGTCGCCGATGGGGGTGTTCTGCTGGTAGTG GTCGGCGAGCTGCACGCTGCCGTCCTCGATGTTGTGGCGGATCTTGAAGTTGGCCTTGATGCCG TTCTTCTGCTTGTCGGCGGTGATATAGACGTTGTGGCTGATGTAGTTGTACTCCAGCTTGTGCCC CAGGATGTTGCCGTCCTCCTTGAAGTCGATGCCCTTCAGCTCGATGCGGTTCACCAGGGTGTCG CCCTCGAACTTCACCTCGGCGCGGGTCTTGTAGTTGCCGTCGTCCTTGAAGAAGATGGTGCGCT CCTGGACGTAGCCTTCGGGCATGGCGGACTTGAAGAAGTCGTGCTGCTTCATGTGGTCGGGGTA GCGGCTGAAGCACTGCACGCCCCAGGTCAGGGTGGTCACGAGGGTGGGCCAGGGCACGGGCAG CTTGCCGGTGGTGCAGATGAACTTCAGGGTCAGCTTGCCGTAGGTGGCATCGCCCTCGCCCTCG CCGGACACGCTGAACTTGTGGCCGTTTACGTCGCCGTCCAGCTCGACCAGGATGGGCACCACCQ CGGTGAACAGCTCCTCGCCCTTGCTCACCATGGTGGCGACCGGTGGATCGTACCGTCGACTCTA GCGGTACCCCGATTGTTTAGCTTGTTCAGCTGCGCTTGTTTATTTGCTTAGCTTTCGCTTAGCGA CGTGTTCACTTTGCTTGTTTGAATTGAATTGTCGCTCCGTAGACGAAGCGCCTCTATTTATACTC CGGCGGTCGAGGGTTCGAAATCGATAAGCTTGGATCCTAATTGAATTAGCTCTAATTGAATTAG TCTCTAATGAATAATCCCCCCTACGCCCCCAACTGAGAGAACTCAAAGGTTACCCCAGTTGGGG CACTACTTCAAGGGTCTGCAGCAAGACTTCGTGCCTAGAGCTCTGCACCGCATCTTCGAGGAGC AGCAGCTGCGGCATGCCGACAAGGTGGCTCTGATCTATCAGCCCAGCACTCCGGGCCAGGGAA TGGCGCCCAGTCAGAGCAGCTACCGCCAGATGAACGAGCGTGCGAACCGGGCAGCCCGCCTCC TGGTGGCCGAGACCCACGGCCGGTTCCTGCAGCCAAACAGCGATGGTGACTTCATCGTGGCTGT GTGCATGCAGCCGTCGGAGGGATTGGTCACCACACTGCTGGCCATCTGGAAGGCTGGCGGCGC GTATTTGCCCATCGATCCCAGCTTCCCGGCGAACCGCATTCACCACATACTGCTGGAGGCGAAG CCCACCTTGGTGATTCGCGACGATGACATCGACGCCGGCCGTTTCCAGGGAACTCCCACGTTAT CCACCACCGAACTGTATGCCAAATCCCTCCAGCTGGCCGGCTCCAATCTGCTCTCAGAGGAGAT GCTGCGCGGTGGCAACGACCACATCGCCATCGTGCTCTACACCTCGGGCAGTACGGGTGTGCCC AAGGGAGTGCGTCTGCCGCACGAGAGCATCCTCAATCGGCTCCAGTGGCAGTGGGCGACCTTT CCGTACACCGCCAACGAGGCGGTGAGCGTTTTCAAGACGGCCTTAACCTTCGTGGACTCGATTG CGGAGCTATGGGGCCCGTTAATGTGTGGCCTGGCCATTCTGGTGGTGCCCAAGGCAGTGACCAA GGATCCCCAGCGATTGGTGGCCCTGTTGGAGCGTTATAAGATCAGGCGTCTCGTACTGGTGCCC ACCCTTCTGCGCTCGTTGCTCATGTATCTGAAGATGGAGGGAGGCGGAGCTGCTCAGAAACTGC 
TGTACAATCTCCAGATTTGGGTCTGCTCCGGAGAACCCCTATCCGTTTCCCTGGCCAGCAGCTT CTTCGACTATTTCGATGAGGGCGTGCACCGGCTGTACAACTTCAATTCGCCCTATAGTGAGTCG TATTACAATTCACTGGCCGTCGTTTTACAACGTCGTGACTGGGAAAACCCTGGCGTTACCCAAO TTAATCGCCTTGCAGCACATCCCCCTTTCGCCAGCTGGCGTAATAGCGAAGAGGCCCGCACCGA TCGCCCTTCCCAACAGTTGCGCAGCCTGAATGGCGAATGGACGCGCCCTGTAGCGGCGCATTAA GCGCGGCGGGTGTGGTGGTTACGCGCAGCGTGACCGCTACACTTGCCAGCGCCCTAGCGCCCG CTCCTTTCGCTTTCTTCCCTTCCTTTCTCGCCACGTTCGCCGGCTTTCCCCGTCAAGCTCTAAATC GGGGGCTCCCTTTAGGGTTCCGATTTAGTGCTTTACGGCACCTCGACCCCAAAAAACTTGATTA GGGTGATGGTTCACGTAGTGGGCCATCGCCCTGATAGACGGTTTTTCGCCCTTTGACGTTGGAG TCCACGTTCTTTAATAGTGGACTCTTGTTCCAAACTGGAACAACACTCAACCCTATCTCGGTCTA TTCTTTTGATTTATAAGGGATTTTGCCGATTTCGGCCTATTGGTTAAAAAATGAGCTGATTTAAC AAAAATTTAACGCGAATTTTAACAAAATTCAGGGCGCAAGGGCTGCTAAAGGAAGCGGAACAC GTAGAAAGCCAGTCCGCAGAAACGGTGCTGACCCCGGATGAATGTCAGCTACTGGGCTATCTG GACAAGGGAAAACGCAAGCGCAAAGAGAAAGCAGGTAGCTTGCAGTGGGCTTACATGGCGATA GCTAGACTGGGCGGTTTTATGGACAGCAAGCGAACCGGAATTGCCAGCTGGGGCGCCCTCTGG TAAGGTTGGGAAGCCCTGCAAAGTAAACTGGATGGCTTTCTTGCCGCCAAGGATCTGATGGCGC AGGGGATCAAGATCTGATCAAGAGACAGGATGAGGATCGTTTCGCATGATTGAACAAGATGGA TTGCACGCAGGTTCTCCGGCCGCTTGGGTGGAGAGGCTATTCGGCTATGACTGGGCACAACAGA CAATCGGCTGCTCTGATGCCGCCGTGTTCCGGCTGTCAGCGCAGGGGCGCCCGGTTCTTTTTGT CAAGACCGACCTGTCCGGTGCCCTGAATGAACTGCAGGACGAGGCAGCGCGGCTATCGTGGCT GGCCACGACGGGCGTTCCTTGCGCAGCTGTGCTCGACGTTGTCACTGAAGCGGGAAGGGACTG GCTGCTATTGGGCGAAGTGCCGGGGCAGGATCTCCTGTCATCCCACCTTGCTCCTGCCGAGAAA GTATCCATCATGGCTGATGCAATGCGGCGGCTGCATACGCTTGATCCGGCTACCTGCCCATTCG ACCACCAAGCGAAACATCGCATCGAGCGAGCACGTACTCGGATGGAAGCCGGTCTTGTCGATC AGGATGATCTGGACGAAGAGCATCAGGGGCTCGCGCCAGCCGAACTGTTCGCCAGGCTCAAGG CGCGCATGCCCGACGGCGAGGATCTCGTCGTGACCCATGGCGATGCCTGCTTGCCGAATATCAT GGTGGAAAATGGCCGCTTTTCTGGATTCATCGACTGTGGCCGGCTGGGTGTGGCGGACCGCTAT CAGGACATAGCGTTGGCTACCCGTGATATTGCTGAAGAGCTTGGCGGCGAATGGGCTGACCGCT TCCTCGTGCTTTACGGTATCGCCGCTCCCGATTCGCAGCGCATCGCCTTCTATCGCCTTCTTGAC GAGTTCTTCTGAATTGAAAAAGGAAGAGTATGAGTATTCAACATTTCCGTGTCGCCCTTATTCC CTTTTTTGCGGCATTTTGCCTTCCTGTTTTTGCTCACCCAGAAACGCTGGTGAAAGTAAAAGATG CTGAAGATCAGTTGGGTGCACGAGTGGGTTACATCGAACTGGATCTCAACAGCGGTAAGATCCT TGAGAGTTTTCGCCCCGAAGAACGTTTTCCAATGATGAGCACTTTTAAAGTTCTGCTATGTGGC GCGGTATTATCCCGTATTGACGCCGGGCAAGAGCAACTCGGTCGCCGCATACACTATTCTCAGA 
ATGACTTGGTTGAGTACTCACCAGTCACAGAAAAGCATCTTACGGATGGCATGACAGTAAGAGA ATTATGCAGTGCTGCCATAACCATGAGTGATAACACTGCGGCCAACTTACTTCTGACAACGATC GGAGGACCGAAGGAGCTAACCGCTTTTTTGCACAACATGGGGGATCATGTAACTCGCCTTGATC GTTGGGAACCGGAGCTGAATGAAGCCATACCAAACGACGAGCGTGACACCACGATGCCTGTAG CAATGGCAACAACGTTGCGCAAACTATTAACTGGCGAACTACTTACTCTAGCTTCCCGGCAACA ATTAATAGACTGGATGGAGGCGGATAAAGTTGCAGGACCACTTCTGCGCTCGGCCCTTCCGGCT GGCTGGTTTATTGCTGATAAATCTGGAGCCGGTGAGCGTGGGTCTCGCGGTATCATTGCAGCAC TGGGGCCAGATGGTAAGCCCTCCCGTATCGTAGTTATCTACACGACGGGGAGTCAGGCAACTAT GGATGAACGAAATAGACAGATCGCTGAGATAGGTGCCTCACTGATTAAGCATTGGTAACTGTCA GACCAAGTTTACTCATATATACTTTAGATTGATTTAAAACTTCATTTTTAATTTAAAAGGATCTA GGTGAAGATCCTTTTTGATAATCTCATGACCAAAATCCCTTAACGTGAGTTTTCGTTCCACTGAG CGTCAGACCCCGTAGAAAAGATCAAAGGATCTTCTTGAGATCCTTTTTTTCTGCGCGTAATCTG CTGCTTGCAAACAAAAAAACCACCGCTACCAGCGGTGGTTTGTTTGCCGGATCAAGAGCTACCA ACTCTTTTTCCGAAGGTAACTGGCTTCAGCAGAGCGCAGATACCAAATACTGTTCTTCTAGTGT AGCCGTAGTTAGGCCACCACTTCAAGAACTCTGTAGCACCGCCTACATACCTCGCTCTGCTAAT CCTGTTACCAGTGGCTGCTGCCAGTGGCGATAAGTCGTGTCTTACCGGGTTGGACTCAAGACGA TAGTTACCGGATAAGGCGCAGCGGTCGGGCTGAACGGGGGGTTCGTGCACACAGCCCAGCTTG GAGCGAACGACCTACACCGAACTGAGATACCTACAGCGTGAGCTATGAGAAAGCGCCACGCTT CCCGAAGGGAGAAAGGCGGACAGGTATCCGGTAAGCGGCAGGGTCGGAACAGGAGAGCGCAC GAGGGAGCTTCCAGGGGGAAACGCCTGGTATCTTTATAGTCCTGTCGGGTTTCGCCACCTCTGA CTTGAGCGTCGATTTTTGTGATGCTCGTCAGGGGGGCGGAGCCTATGGAAAAACGCCAGCAAC GCGGCCTTTTTACGGTTCCTGGCCTTTTGCTGGCCTTTTGCTCACATGTTCTTTCCTGCGTTATC CCCTGATTCTGTGGATAACCGTATTACCGCCTTTGAGTGAGCTGATACCGCTCGCCGCAGCCGA ACGACCGAGCGCAGCGAGTCAGTGAGCGAGGAAGCGGAAGAGCGCCCAATACGCAAACCGCCT CTCCCCGCGCGTTGGCCGATTCATTAATGCAGCTGGCACGACAGGTTTCCCGACTGGAAAGCGG GCAGTGAGCGCAACGCAATTAATGTGAGTTAGCTCACTCATTAGGCACCCCAGGCTTTACACTT TATGCTTCCGGCTCGTATGTTGTGTGGAATTGTGAGCGGATAACAATTTCACACAGGAAACAGC TATGACCATGATTACGCCAAGCTATTTAGGTGACACTATAGAATACTCAA 


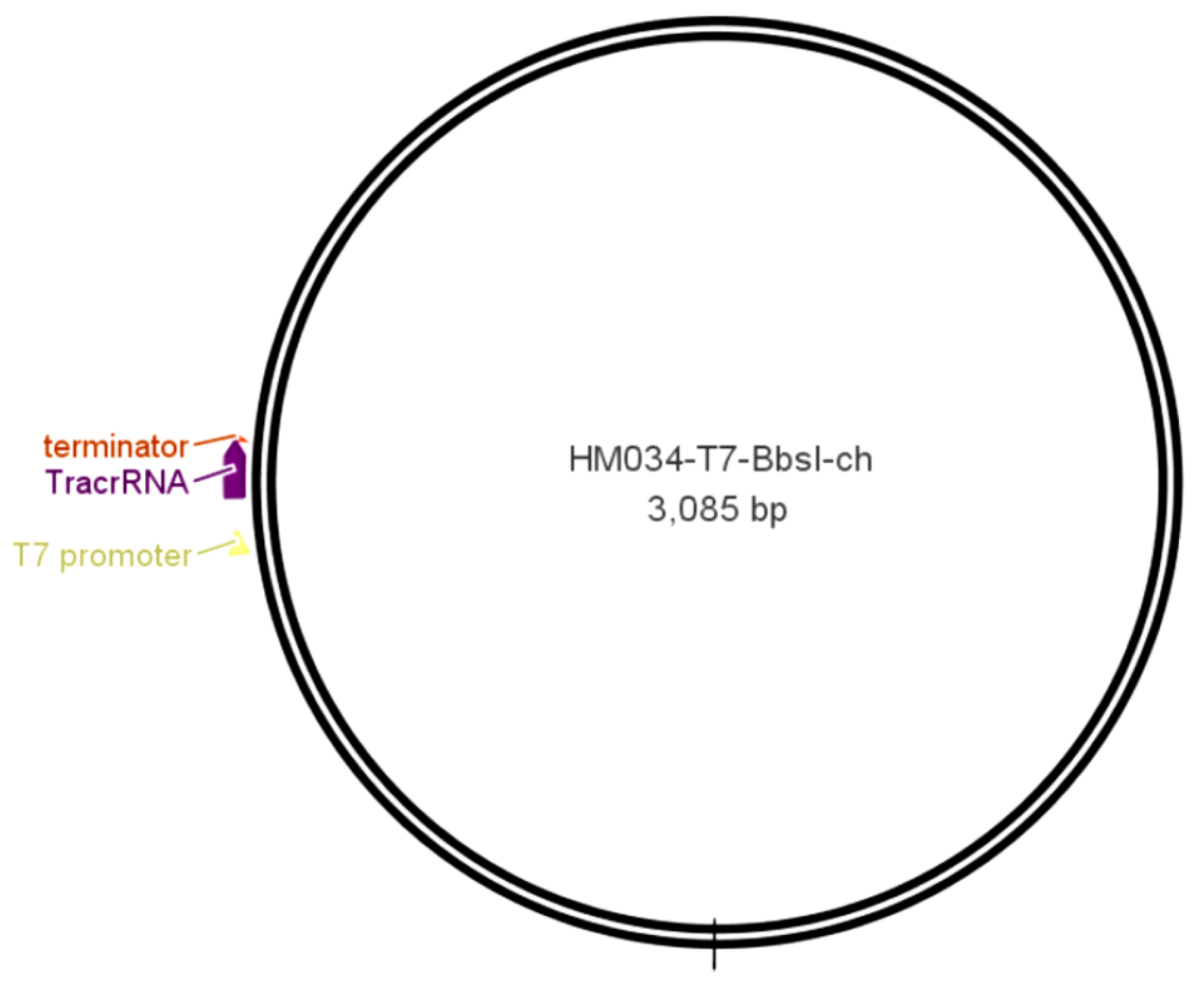

\section{> HM034-T7-BbsI-ch}

CACCTGACGCGCCCTGTAGCGGCGCATTAAGCGCGGCGGGTGTGGTGGTTACGCGCAGCGTGA CCGCTACACTTGCCAGCGCCCTAGCGCCCGCTCCTTTCGCTTTCTTCCCTTCCTTTCTCGCCACG TTCGCCGGCTTTCCCCGTCAAGCTCTAAATCGGGGGCTCCCTTTAGGGTTCCGATTTAGTGCTTT ACGGCACCTCGACCCCAAAAAACTTGATTAGGGTGATGGTTCACGTAGTGGGCCATCGCCCTGA TAGACGGTTTTTCGCCCTTTGACGTTGGAGTCCACGTTCTTTAATAGTGGACTCTTGTTCCAAAC TGGAACAACACTCAACCCTATCTCGGTCTATTCTTTTGATTTATAAGGGATTTTGCCGATTTCGG CCTATTGGTTAAAAAATGAGCTGATTTAACAAAAATTTAACGCGAATTTTAACAAAATATTAAC GCTTACAATTTCCATTCGCCATTCAGGCTGCGCAACTGTTGGGAAGGGCGATCGGTGCGGGCCT CTTCGCTATTACGCCAGCTGGCGAAAGGGGGATGTGCTGCAAGGCGATTAAGTTGGGTAACGC CAGGGTTTTCCCAGTCACGACGTTGTAAAACGACGGCCAGTGAATTGTAATACGACTCACTATA GGGCGAATTGGGTACCGGGCCCCCCCTCGAGGTCGACGGTATCGATAAGCTTGATGTGAATTGT AATACGACTCACTATAGGGTCTTCGAGAAGACCTGTTTTAGAGCTAGAAATAGCAAGTTAAAAT AAGGCTAGTCCGTTATCAACTTGAAAAAGTGGCACCGAGTCGGTGCTTTTTTAAATCGAATTCC TGCAGCCCGGGGGATCCACTAGTTCTAGAGCGGCCGCCACCGCGGTGGAGCTCCAGCTTTTGTT CCCTTTAGTGAGGGTTAATTTCGAGCTTGGCGTAATCATGGTCATAGCTGTTTCCTGTGTGAAAT TGTTATCCGCTCACAATTCCACACAACATACGAGCCGGAAGCATAAAGTGTAAAGCCTGGGGTG CCTAATGAGTGAGCTAACTCACATTAATTGCGTTGCGCTCACTGCCCGCTTTCCAGTCGGGAAA CCTGTCGTGCCAGCTGCATTAATGAATCGGCCAACGCGCGGGGAGAGGCGGTTTGCGTATTGG GCGCTCTTCCGCTTCCTCGCTCACTGACTCGCTGCGCTCGGTCGTTCGGCTGCGGCGAGCGGTA 
TCAGCTCACTCAAAGGCGGTAATACGGTTATCCACAGAATCAGGGGATAACGCAGGAAAGAAC ATGTGAGCAAAAGGCCAGCAAAAGGCCAGGAACCGTAAAAAGGCCGCGTTGCTGGCGTTTTTC CATAGGCTCCGCCCCCCTGACGAGCATCACAAAAATCGACGCTCAAGTCAGAGGTGGCGAAAC CCGACAGGACTATAAAGATACCAGGCGTTTCCCCCTGGAAGCTCCCTCGTGCGCTCTCCTGTTC CGACCCTGCCGCTTACCGGATACCTGTCCGCCTTTCTCCCTTCGGGAAGCGTGGCGCTTTCTCAT AGCTCACGCTGTAGGTATCTCAGTTCGGTGTAGGTCGTTCGCTCCAAGCTGGGCTGTGTGCACG AACCCCCCGTTCAGCCCGACCGCTGCGCCTTATCCGGTAACTATCGTCTTGAGTCCAACCCGGT AAGACACGACTTATCGCCACTGGCAGCAGCCACTGGTAACAGGATTAGCAGAGCGAGGTATGT AGGCGGTGCTACAGAGTTCTTGAAGTGGTGGCCTAACTACGGCTACACTAGAAGGACAGTATTT GGTATCTGCGCTCTGCTGAAGCCAGTTACCTTCGGAAAAAGAGTTGGTAGCTCTTGATCCGGCA AACAAACCACCGCTGGTAGCGGTGGTTTTTTTGTTTGCAAGCAGCAGATTACGCGCAGAAAAAA AGGATCTCAAGAAGATCCTTTGATCTTTTCTACGGGGTCTGACGCTCAGTGGAACGAAAACTCA CGTTAAGGGATTTTGGTCATGAGATTATCAAAAAGGATCTTCACCTAGATCCTTTTAAATTAAAA ATGAAGTTTTAAATCAATCTAAAGTATATATGAGTAAACTTGGTCTGACAGTTACCAATGCTTAA TCAGTGAGGCACCTATCTCAGCGATCTGTCTATTTCGTTCATCCATAGTTGCCTGACTCCCCGTC GTGTAGATAACTACGATACGGGAGGGCTTACCATCTGGCCCCAGTGCTGCAATGATACCGCGAG ACCCACGCTCACCGGCTCCAGATTTATCAGCAATAAACCAGCCAGCCGGAAGGGCCGAGCGCA GAAGTGGTCCTGCAACTTTATCCGCCTCCATCCAGTCTATTAATTGTTGCCGGGAAGCTAGAGT AAGTAGTTCGCCAGTTAATAGTTTGCGCAACGTTGTTGCCATTGCTACAGGCATCGTGGTGTCA CGCTCGTCGTTTGGTATGGCTTCATTCAGCTCCGGTTCCCAACGATCAAGGCGAGTTACATGAT CCCCCATGTTGTGCAAAAAAGCGGTTAGCTCCTTCGGTCCTCCGATCGTTGTCAGAAGTAAGTT GGCCGCAGTGTTATCACTCATGGTTATGGCAGCACTGCATAATTCTCTTACTGTCATGCCATCCG TAAGATGCTTTTCTGTGACTGGTGAGTACTCAACCAAGTCATTCTGAGAATAGTGTATGCGGCG ACCGAGTTGCTCTTGCCCGGCGTCAATACGGGATAATACCGCGCCACATAGCAGAACTTTAAAA GTGCTCATCATTGGAAAACGTTCTTCGGGGCGAAAACTCTCAAGGATCTTACCGCTGTTGAGAT CCAGTTCGATGTAACCCACTCGTGCACCCAACTGATCTTCAGCATCTTTTACTTTCACCAGCGTT TCTGGGTGAGCAAAAACAGGAAGGCAAAATGCCGCAAAAAAGGGAATAAGGGCGACACGGAA ATGTTGAATACTCATACTCTTCCTTTTTCAATATTATTGAAGCATTTATCAGGGTTATTGTCTCAT GAGCGGATACATATTTGAATGTATTTAGAAAAATAAACAAATAGGGGTTCCGCGCACATTTCCC CGAAAAGTGC 